Portland State University

PDXScholar

Spring 5-18-2015

\title{
The Impact of Communication Impairments on the Social Relationships of Older Adults
}

Andrew Demetrius Palmer

Portland State University

Follow this and additional works at: https://pdxscholar.library.pdx.edu/open_access_etds

Part of the Communication Commons

Let us know how access to this document benefits you.

\section{Recommended Citation}

Palmer, Andrew Demetrius, "The Impact of Communication Impairments on the Social Relationships of Older Adults" (2015). Dissertations and Theses. Paper 2344.

https://doi.org/10.15760/etd.2341

This Dissertation is brought to you for free and open access. It has been accepted for inclusion in Dissertations and Theses by an authorized administrator of PDXScholar. For more information, please contact pdxscholar@pdx.edu. 
The Impact of Communication Impairments on the Social Relationships of Older Adults

by

Andrew Demetrius Palmer

A dissertation submitted in partial fulfillment of the requirements for the degree of

\author{
Doctor of Philosophy \\ in \\ Urban Studies
}
Dissertation Committee:
Jason T. Newsom, Chair
Paula C. Carder
Diana L. White
Gabrielle Saunders
Hyeyoung Woo

Portland State University

2015 


\begin{abstract}
Communication forms the foundation of social interaction. For older adults, however, there is known to be an increased risk of developing conditions that interfere with the ability to communicate. These conditions may occur for a variety of reasons, including age-related changes in physical or sensory functioning, injury, and disease. It is estimated that $55 \%$ of all Medicare beneficiaries have a communication impairment of some kind. Social contact is known to be vital for older adults' mental and physical health but, because communication impairments often co-occur with other types of disability, it is difficult to generalize about the relative impact of a communication impairment on the social relationships of older adults. Specific aims of the study were to examine whether the severity of a communication impairment is associated with social measures, whether there is an interaction between communication impairments and physical disability, and to examine the role of relationship-control strategies in maintaining access to a larger or more supportive social network. A mixed-methods study design was employed. Community-dwelling older adults were surveyed about the size and diversity of their social networks, frequency of social interactions, and physical and mental health $(\mathrm{n}=$ 240) and qualitative data were collected from a smaller subsample. Findings demonstrated that communication impairment was a significant independent predictor for key characteristics of social relationships, including the composition of the social network, certain types of social support, the frequency of social participation, and social self-efficacy. Communication impairment was a significant predictor for higher levels of loneliness and depression. In addition two distinct pathways between communication
\end{abstract}


impairment and psychological well-being were identified, with social self-efficacy and reassurance of worth as mediators. Additional insights were provided by the qualitative results. These findings may guide future clinical practice and research by providing a better understanding of the role of communication in health, disability, and the risk of social isolation. 


\section{Dedication}

I would like to dedicate this work to my parents, to my wife Kristine, to the many patients that I have learned so much from over the years, and also to the memory of Rob Williams whose untimely loss is still a source of sadness. 


\section{Acknowledgments}

In looking back, I was pleasantly surprised to realize that the roots of this project go back a long way. In rereading the abstract of an article based on my Master's thesis I came across this quote, which I had forgotten: "The ability to communicate showed an inverse relationship with feelings of social isolation." I'd like to thank Minnie Graham for inviting me to explore the field of Speech Pathology originally and also for mentoring me in the completion of a thesis, which started me along this path to a $\mathrm{PhD}$.

It is also worth acknowledging that no one accomplishes these things in isolation. First and foremost, let me thank all of the study participants who contributed their time and personal experiences to this research. I'd also like to thank many people for their help and guidance along the way: Judie Rau for telling me about the Urban Studies PhD originally; Margaret Neal for supporting my application and introducing me to the field of Gerontology; the members of my PhD support group, Barbara Shields, Rob Williams, and Zeljka Carol Kekez; Gretchen Luhr, Cynthia Lopez, and Tracy Braden for suggestions, support, and practical advice; David Kinsella and the students in his $\mathrm{PhD}$ seminar where the original ideas for this project were developed; the faculty of the Urban Studies department, the Institute on Aging, and other departments at PSU for allowing me to put together a course of study and utilize resources from a wide variety of fields; my Comps committee Jason Newsom, Diana White, and Gary Brodowicz for allowing me to explore ideas that formed the foundation of the dissertation; Jason Newsom, Dara Sorkin, and Karen Rook for allowing me to use the data from the Later Life Study of Social Exchanges for preliminary work in this area; Jane, Pete, and Paul who proofed 
early versions of the study materials and instruments; staff at the National Association for the Deaf, National Multiple Sclerosis Society, the National Spasmodic Dysphonia Association and Pat Wertz Sanders at WebWhispers for publicizing the study; my Dissertation Committee for their time and effort in reading my many overlong draft documents and providing ever-insightful suggestions and feedback; and all of the people that I have asked for advice or help or bored along the way.

I am deeply, deeply indebted to the Department of Otolaryngology at Oregon Health and Science University for their support during my completion of this work and my colleagues at the Northwest Clinic for Voice \& Swallowing for covering patient care, teaching, research, supervision, and so many other things while I was working on the PhD. Thanks most especially to Donna Graville for her tireless support, advice, and encouragement, without which I would not have made it through. I would also like to thank my parents and in particular my Mum whose experiences with hearing loss first motivated me to consider a career in this area, as well as the many patients and clients that I have seen and learned from over the years. Thanks above all to my wife, Kristine, for her ongoing support over the course of this long, long journey.

And following its path, we took no care

To rest, but climbed: he first, then I, so far,

Through a round aperture I saw appear

Some of the beautiful things that Heaven bears,

Where we came forth, and once more saw the stars.

- Dante Alighieri 
Table of Contents

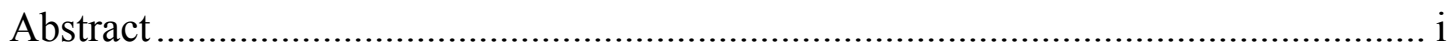

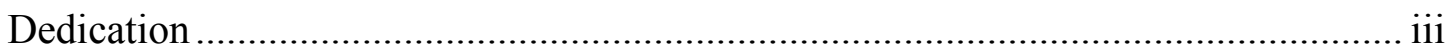

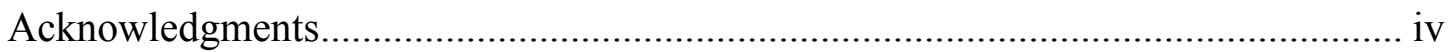

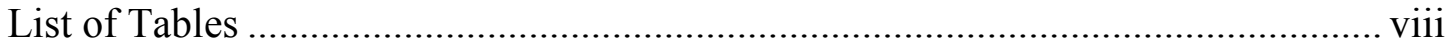

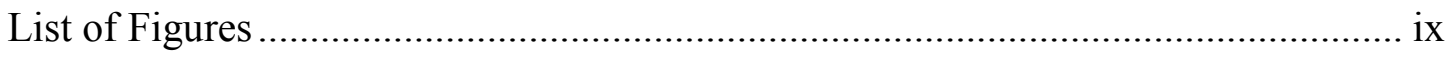

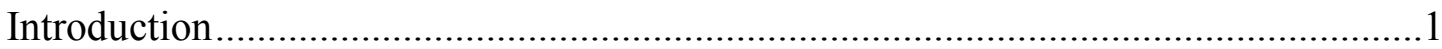

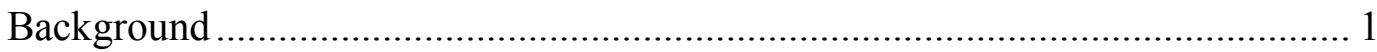

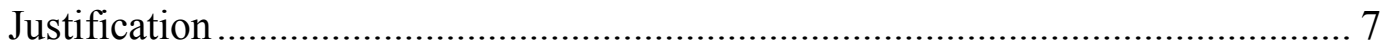

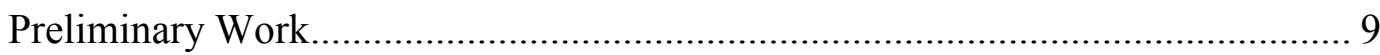

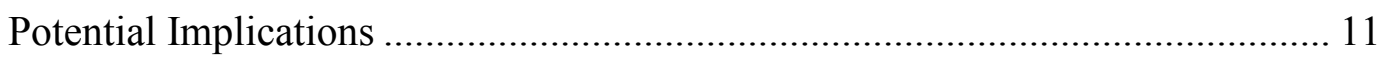

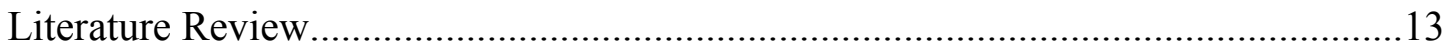

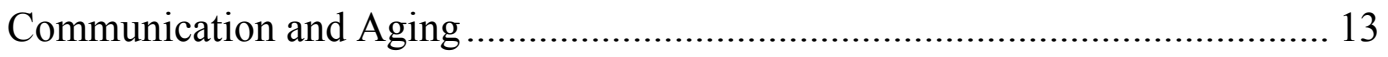

Theoretical Frameworks for Understanding the Impact of Communication Impairment .................................................................................. 25

The Importance of Social Relationships for Mental and Physical Health ............ 42

Defining and Measuring Social Relationships................................................ 45

Theories of Social Relationships and Aging................................................. 59

The Impact of Communication Impairment on Social Relationships.................. 63

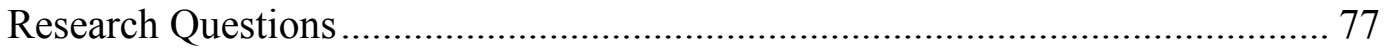

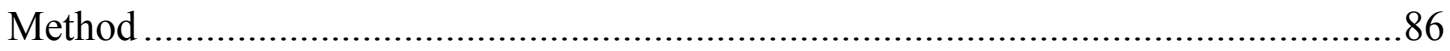

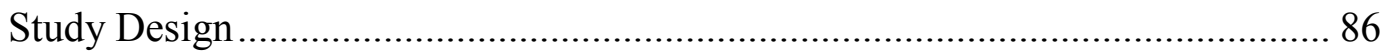

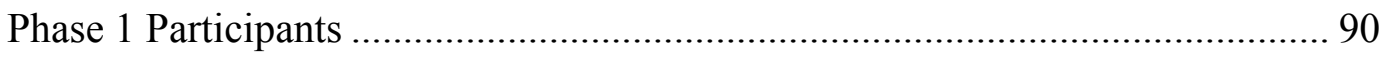

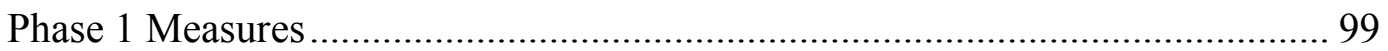

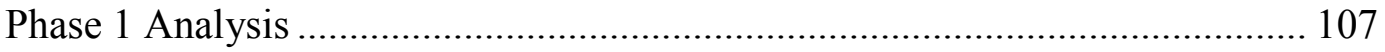

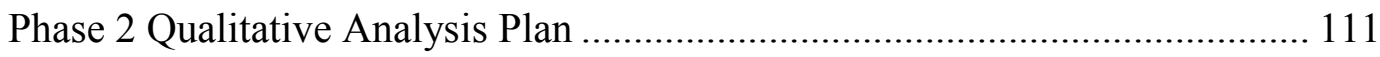

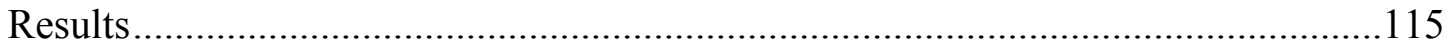

Analysis Overview .................................................................................... 115

Descriptive Characteristics of Phase 1 Participants ...................................... 115

Reliability Analyses ............................................................................. 118

Regression Analyses: Predicting Social and Psychological Characteristics....... 124

Physical Disability, Social Support, and Social Self-Efficacy as

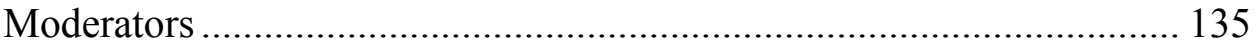

Social Relationship Variables as Mediators of Psychological Well-Being ........ 140

Perceived Changes in Social Self-Efficacy...................................................... 145

Phase 2 Qualitative Investigation Results .................................................... 146

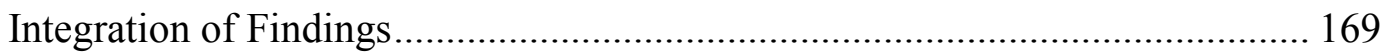

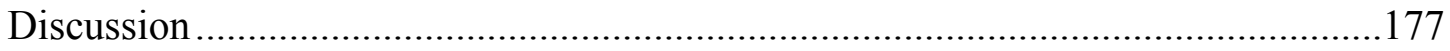




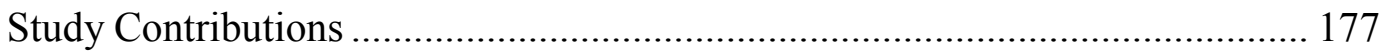

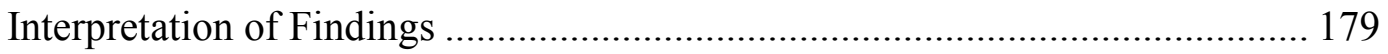

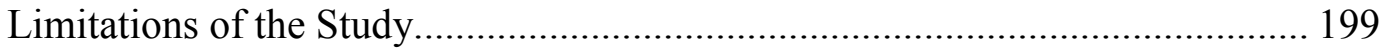

Clinical Implications and Suggestions for Future Research ........................... 200

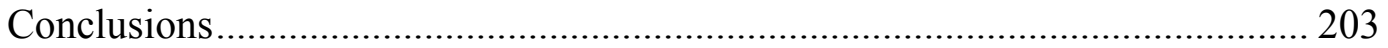

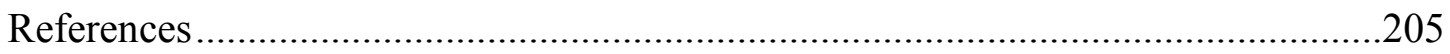

Appendix A. Summary of Published Instruments ....................................................237

Appendix B. Published Instruments Related to Disability ......................................240

Appendix C. Published Instruments Related to Mental Health \& Well-Being .........244

Appendix D. Published Instruments Related to Social Relationships ......................247

Appendix E. Evidence for Content Validity of the CETI-M ...................................255

Appendix F. Open-Ended Questions for the Individual Interviews .........................257

Appendix G: Study Approval Documentation from OHSU and PSU ....................259

Appendix H. Approved Study Documents .............................................................262

Appendix I: Identification and Exclusion of Potential Participants...........................278

Appendix J: Categorization of Open-Ended Responses from Phase 1 ......................281

Appendix K. Additional Descriptive Data from Phase 1.........................................286 


\section{List of Tables}

Table 1. A Summary of Measures Used to Assess Social Relationships in the Health Literature

Table 2. Comparing Component Models of Social Support Across Authors................... 54

Table 3. A Summary of Recruitment Methods for Phases 1 and 2................................ 89

Table 4. Response Rate by Group for Targeted Participants............................................ 97

Table 5. Number of Responses Included in the Final Sample by Group for All Participants 98

Table 6. Calculated Sample Sizes for Various Effect Sizes in the Final Regression Model

Table 7. Age and Health Characteristics of All Participants .......................................... 116

Table 8. Other Health and Demographic Characteristics of Participants ....................... 117

Table 9. Mean, Skewness, Kurtosis, and Internal Reliability for Composite Measures and Scales

Table 10. Correlation among Study Measures............................................................ 126

Table 11. Mean Communication Effectiveness Scores by Subgroup............................. 126

Table 12. Multiple Regressions for Social and Psychological Variables ........................ 127

Table 13. Mediation of the Effect of Communication Effectiveness on Loneliness through Social Characteristics in a Simultaneous Model.

Table 14. Mediation of the Effect of Communication Effectiveness on Depression through Social Characteristics in a Simultaneous Model ......................................... 144

Table 15. Descriptive Characteristics of Qualitative Interview Participants 147

Table 16. Comments by Interview Participants about Changes in Social Characteristics

Table 17. Summary of Themes and Subthemes from the Qualitative Interviews 149 


\section{List of Figures}

Figure 1. Differences between a medical model (a) and a biopsychosocial model (b) of

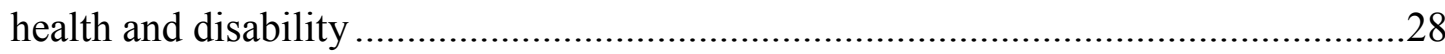

Figure 2. A conceptual model of the relationship between communication and quality of life .30

Figure 3. A conceptual framework for the impact of social networks on health. .50

Figure 4. Hypothesized moderating effect of physical disability on psychological wellbeing.

Figure 5. Hypothesized moderating effect of social support on psychological wellbeing. .80

Figure 6. Hypothesized mediating effect of social relationships on psychological wellbeing.

Figure 7. Hypothesized moderating effect of relationship control on social network size and social support

Figure 8. Summary schematic of the relationships between variables explored in the study

Figure 9. Histogram of communication effectiveness scores on the CETI-M

Figure 10. Association between communication effectiveness scores on the CETI-M and the number of functional limitations.

Figure 11. Simple slopes for social network size on communication at three values of social self-efficacy

Figure 12. Full mediation 140

Figure 13. Partial mediation 140

Figure 14. Illustration of the partial mediational model for loneliness 143

Figure 15. Illustration of the full mediation model for depression 144 


\section{Introduction}

\section{Background}

Communication forms the foundation of social interaction (Heine \& Browning, 2002). In older adults, communication is central to the process of successfully adjusting and adapting to the aging process, being essential for living independently, pursuing personal goals and interests, performing social roles and functions, maintaining personal and familial relationships, making decisions, and exercising control over quality of life and care (Lubinski \& Welland, 1997). Studies of communication in normal aging have shown that the conversational skills of normally aging older adults tend to remain wellpreserved, even though the semantic content and syntactic structure of language use change over the life-course (Shadden, 1997). With increasing age, however, there is an increase in the prevalence of conditions that may interfere with communication. The conditions that cause communication impairments vary widely in their type and severity, as well as in their co-occurrence with other types of disability and functional limitations (Yorkston, Bourgeois, \& Baylor, 2010). Hearing impairment is the most prevalent type of communication impairment nationally and is the third most common chronic condition of older adults (Wallhagen, 2002). The prevalence of hearing impairment increases steadily with increasing age from $45 \%$ of those in their sixties to $89 \%$ of those aged 80 or more (Lin, Niparko, \& Ferrucci, 2010). The prevalence of other types of communication impairments is less well-known. In one large-scale survey of Medicare beneficiaries, it was estimated that $55 \%$ of all Medicare beneficiaries (more than 16 million) had a communication impairment of some kind (Hoffman et al. 2005). 
There is a significant body of evidence that the quantity and quality of an individual's social relationships are associated with better physical and mental health across the life-course (Holt-Lunstad, Smith, \& Layton, 2010; House, Landis, \& Umberson, 1988). In studies of older adults, psychological outcomes are associated not just with the size but also the composition of the individual's social network. Older adults with the most restricted social networks have the highest levels of depressive symptoms, with the most negative outcomes for those with a paucity of friends (Fiori, Antonucci \& Cortina, 2006). These findings reinforce the importance of companionship for positive mental health. Previous research has shown that family and friends frequently serve very different functions in the lives of older people (Crohan \& Antonucci, 1989). In particular, friends seem to be particularly important for feelings of emotional well-being and selfesteem (Johnson \& Catalano, 1983; Lee, 1979; Rook, 1987; Wood \& Robertson, 1978) whereas family are more important for providing practical and material assistance that may be critical for delaying or preventing institutionalization (Tobin \& Kulys, 1981).

There are a number of ways of measuring an individual's social relationships can be defined and measured. There are many different definitions and categorizations of "social support" and no one model is universally accepted but the term typically refers to the different functions that social relationships may perform, which includes the provision of emotional support, as well as practical and informational assistance, as well as a sense of belonging to a social group or community (Uchino, 2006). In general, most authors distinguish between the terms "loneliness" and "social isolation." Loneliness is generally considered to be a subjective perception of inadequacy in the nature of one's social relationships, regardless of the number or nature of one's social contacts. In 
contrast, social isolation is a more objective measure which relates to shortcomings in the size or quality of an individual's social network (de Jong Giervald \& Havens, 2004). The term "social network" refers to the web of social relationships that surround an individual as well as the characteristics of those ties and typically includes relationships with friends, family members, neighbors, work associates, or other important individuals in that person's life (Berkman \& Glass, 2000; Bulmer, 1987). Studies of normal aging have shown that changes in social relationships occur across the life-course. Stereotypical views had long depicted old age as a time of social isolation and loss, but more recent research indicates that this is not typical. According to Socioemotional Selectivity Theory, older adults choose to maintain social relationships that are most rewarding and gradually abandon those which are less so (Carstensen, Isaacowitz, \& Charles, 1999). As a result, the total number of social relations decreases with age, but the number of close social relationships does not and social support remains stable until very old age. Largescale, national studies have found that the typical social network of older adults ranges between seven and ten people (Antonucci \& Akiyama, 1987; Mugford \& Kendig, 1987; Phillipson, Bernard, Phillips, \& Ogg, 1998; Smith \& Baltes, 1998). Evidence suggests that only a minority of community-dwelling older adults is "severely" lonely or isolated, with most estimates in the region of $10 \%$ (Edelbrock, Buys, Creasey, \& Broe, 2001; Grenade \& Boldy, 2008; Hawthorne, 2008; Victor, Scambler, Bond, \& Bowling, 2000). Risk factors for loneliness and isolation include widowhood or loss of a partner, having no (surviving) children, living alone, deteriorating health, and significant negative life events. The risk of each of these life events occurring increases over time and, 
consequently, the risk of loneliness and social isolation increases with advancing age (Dugan \& Kivett, 1994).

To date, changes in social networks in individuals with communication impairments have not been extensively investigated (Worrall \& Hickson, 2003). In studies of community-dwelling older adults, communication measures have been found to have weak associations with social characteristics such as social network size (Hickson, Worrall, Barnett, \& Yiu, 1995; Lind et al., 2003). In contrast, there is a wealth of qualitative and descriptive literature relating to the ability to maintain personal relationships in individuals with communication impairments of varying kinds. Studies of communication impairments that are congenital or occur early in life have shown that there are long-term impacts on the formation and maintenance of social relationships (Ballin \& Balandin, 2007; McCormack, McLeod, McAllister, \& Harrison, 2009). Studies of conditions that occur in mid- to late-life have also shown the social impact of communication difficulties in older adults (Bringfelt, Hartelius, \& Runmarker, 2006; Starks, Morris, Yorkston, Gray \& Johnson, 2010). These changes may also have a negative impact on an individual's social network, as has been found in the case of older stroke survivors with aphasia (Davidson, Howe, Worrall, Hickson, \& Togher, 2008; Hilari \& Northcott, 2006; Parr, 2007). There is some evidence that communication impairments may have a deleterious impact on social support over time. Hearing impairment has been shown to be associated with decreased social support over time in a longitudinal study of women, even after controlling for a variety of health and demographic characteristics (Pachana, Smith, Watson, McLaughlin \& Dobson, 2008). Collectively, these findings suggest that the changes in the social networks of older adults 
with communication impairments may be very different from those observed in normal aging. Social networks may diminish over time as they are abandoned by former friends (i.e. the process is not elective) or due to the fact that individuals may choose to avoid social situations and activities in which they have trouble communicating due to fears of ridicule and stigma, leading to reduced social participation, loneliness, social isolation and withdrawal (Hétu, Jones \& Getty, 1993).

In addition to the body of work related to the positive impact of social support and social networks on physical and mental health, there has also been a growing interest in negative social interactions. These types of interactions include a lack of support when needed, unwanted advice/intrusion, criticism, rejection and neglect. Negative social interactions have been found to have an adverse impact on physical and mental health (Krause \& Jay, 1991; Rook, 1998), and in some cases that the toll of negative interactions has been found to outweigh the benefits of positive interactions (Newsom, Nishishiba, Morgan, \& Rook, 2003; Okun, Melichor, \& Hill, 1990). There is good reason to suppose that communication impairments may cause an increase in negative social interactions. In progressive conditions, the impact of worsening communication has been found to be associated with a steady decline in the quality of intimate relationships (Baikie, 2002; Carter et al., 1998). Studies of couples in which one partner has a hearing impairment have shown that there are numerous negative impacts on communication (Anderson \& Noble, 2005; Heine, Erber, Osborn, Browning, 2002; Hétu et al., 1993; Preminger \& Meeks, 2010; Scarinci, Worrall, \& Hickson, 2008). Further, the impact of the use of hearing aids has also been shown to have positive impacts not just on the individual with hearing loss but also on their partners and family members, resulting in greater social 
participation, more interpersonal warmth, fewer communication difficulties, decreased negative emotions, better emotional stability, and decreased caregiver burden and distress (Boi et al., 2012; Kochkin \& Rogin, 2000; Tolson, Swan, \& Knussen, 2002).

Another area of research relates to social participation. The World Health Organization has targeted the enhancement of social participation by older adults as part of its policy framework in addressing concerns about population aging (WHO, 2002). With regard to communication impairments, the findings from previous research have been mixed. In studies of community-dwelling older adults, communication-related measures have not been proven to be predictive of social participation (Cruice, Worrall, \& Hickson, 2005). This lack of an association is in contrast to the findings from clinical populations, where associations between communication impairment and participation have been reported (Baylor, Yorkston, Bamer, Britton, \& Amtmann, 2010; Mulrow, Aguilar, Endicott, Tuley, et al., 1990).

It is difficult to make general statements about the social impact of a communication impairment in older adults, however, for a number of reasons. It is difficult to estimate the relative impact of a communication disorder because they often co-occur with other health problems and functional limitations, including increased age, poorer health, and higher levels of functional disability (Hoffman et al., 2005; Yorkston et al., 2010). Consequently, it is difficult to isolate the relative contribution of the communication impairment itself from other aspects of disease, such as physical limitations (Bringfelt et al., 2006; Kauhanen et al., 1999). In addition, most studies tend to study one type of communication impairment (e.g. speech or hearing) in isolation rather than considering a broader definition of communication deficits. The current study 
was undertaken to investigate the following questions: Are communication impairments associated with the social function of community-dwelling older adults, even after controlling for other health and demographic factors? If so, are all aspects of social functioning affected equally or are some aspects of social interaction affected disproportionately? Is there evidence for an interaction between different types of disability, such as between communication impairments, visual impairment and/or physical disability? Finally, what characteristics appear to mediate or moderate the relationship between communication impairments and psychological well-being that might allow generation of a hypothetical causal model?

\section{Justification}

It is not known how communication disabilities affect the social lives of older adults. While there is some evidence that overall network size may diminish over time as individuals age (Davidson et al., 2008; Hilari \& Northcott, 2006), there is also qualitative evidence to suggest that some close personal relationships may intensify (Fletcher, Cohen, Schumacher, \& Lydiatt, 2010). Social networks that are primarily composed of kin are associated with poorer psychological outcomes both for older adults generally and also those with disabilities (Felton \& Berry, 1992; Fiori et al., 2006; McIlvane \& Reinhardt, 2001). It is not known whether this is also the case for older adults with communication impairments. Some authors have speculated that smaller networks might be preferable in some situations. For example, Lind and colleagues cite Bowling's work and speculate that older adults with sensory impairments might prefer smaller networks due to a reduction in the "pressures and responsibilities that can be associated with having a large number of relationships" (2003, p. 24). It is also possible that withdrawing 
from the social network may constitute a form of protective function as the individuals seek to insulate themselves from negative encounters and interactions. Other authors have found that social withdrawal can be used as a protective strategy for avoiding conflict (e.g. Morgan, 1989). Consequently, the association between network size and composition and its implications for mental health and well-being is not well-understood. By using well-validated measures of communication, social relationships and mental health, the current study will seek to examine the relationship between these factors in greater and gain a better understanding of how they are connected.

Previous studies have shown that social support may have a buffering effect on negative mental health outcomes but the data from older adults with communication impairments have been inconsistent (Frankel \& Turner, 1983; Oppegard et al., 1984; Ormel et al., 1997). There is also some evidence that communication impairments have a deleterious effect on social support over time even after controlling for other predictors (Pachana et al., 2008). As a result, it is not clear the extent to which social support may moderate or mediate the relationship with variables such as depression, loneliness and life satisfaction.

Some authors have argued that older adults employ "proactive aging" to negotiate relationship losses and meet their emotional and physical needs (Cantor, 1979, 1980; Lang \& Carstensen, 1994). It is not known whether older adults with communication impairments may be able to compensate for their deficits and maintain their social network and its associated support through relationship control and management activities at the individual level, such as through social self-efficacy. According to the "support-efficacy model" in which it has been hypothesized that self-efficacy may help to 
explain the association between social relationships and well-being (Antonucci \& Jackson, 1987). Although this concept has not been studied with regard to either older adults or those with a communication impairment, social self-efficacy has previously been shown to be a significant predictor of mental health in older adults (Fiori et al., 2006).

The WHO has targeted the enhancement of social participation by older adults as part of its policy framework in addressing concerns about population aging (WHO, 2002). Despite considerable research in this area, however, there are wide variations in the definition of the concept of social participation (Levasseur, Richard, Gauvin, \& Raymond, 2010) as well as a lack of consensus about which published measures may be the most appropriate outcome tool for individuals with various kinds of disabilities (Dalemans, de Witte, Lemmens, van den Heuvel, \& Wade, 2008; Dijkers, Whiteneck \& El-Jaroudi, 2000). With regard to adults with communication impairments, findings from previous studies have been mixed. One study found no relationship between objective measures of hearing and naming ability and either communicative or social participation in a community-dwelling sample of older adults (Cruice et al., 2005). A study of individuals with multiple sclerosis, however, found that subjective measures of speech difficulties were significant predictors of communicative participation (Baylor et al., 2010).

\section{Preliminary Work}

Preliminary work has begun to address some of these questions using data from a representative national sample of community-dwelling adults aged 65 and older living in the continental United States. To date, most large-scale studies of older adults have 
examined the impact of hearing impairment but have not considered a more general definition of communication impairments in their predictive models. Consequently a preliminary analysis was undertaken to investigate the following questions: 1) Are communication impairments associated with the social relationships of communitydwelling older adults, even after controlling for other health and demographic factors? 2) If so, are all aspects of social relationships affected equally or are some aspects of social interaction affected disproportionately?

The data for the analysis came from the Later Life Study of Social Exchanges (LLSSE) a 2-year, five-wave longitudinal study of older adults (Sorkin \& Rook, 2004). Using data from one wave of this of this study $(\mathrm{n}=742)$ a secondary analysis was performed (Palmer, Newsom \& Rook, 2012). A communication impairment score was created by averaging scores from three survey items: a) difficulty using the telephone; $b$ ) difficulty hearing (even with a hearing aid); and, c) difficulty understanding and responding quickly to questions. Results from a series of multiple regressions indicated that communication impairment was significantly associated with several aspects of social relationships even after controlling for age, gender, partnership status, health, and functional limitations. Specifically, communication impairment was a significant predictor of loneliness, fewer positive social exchanges, smaller network size, and fewer social activities. Surprisingly, communication impairment was not a significant predictor of negative social exchanges. In keeping with theories such as Strength and Vulnerability Integration and Socioemotional Selectivity Theory, it is known that older adults are more susceptible to the physiological impact of stress and will work harder to avoid or prevent conflict in their interpersonal relationships (Almeida, Piazza, Stawski, \& Klein, 2011). 
Thus, one possible explanation of these findings is that, in order to avoid negative social exchanges, withdrawing from the social network may constitute a form of protective function as the individuals seek to insulate themselves from negative encounters and interactions. Evidence of the negative implications of social support has been found in other populations with the consequence that social withdrawal is a protective strategy for avoiding conflict (e.g. Morgan, 1989). If so, it is possible that avoidance and social withdrawal may be deliberate but could have negative long-term implications for mental or physical health, or place individuals at greater risk of social isolation or institutionalization.

These findings must be interpreted with caution for a number of reasons. First, in general very few people in the dataset had communication difficulty that was more than mild. Second, the presence of a communication impairment was based on a combined variable that was novel and could not be validated by other information about the participants' communication status. Third, the internal reliability of the communication measure was low. Consequently, these findings need to be validated and explored in greater detail with individuals known to have a documented communication disorder and using previously-validated measurement tools.

\section{Potential Implications}

Currently it is not well understood how communication impairments affect the social relationships of older adults and, in turn, whether individuals with communication impairments are at greater risk of depression, social isolation, reduced social support or institutionalization. Because research is limited in this area, it is also not known to what extent there are strategies that may enable older adults to maintain social networks more 
effectively, minimize the impact of their loss, or help to rebuild the network and regain losses over time. A better understanding of which social relationships are affected and in what ways might guide future research relating to interventions that reduce the severity of a communication impairment or enable an individual to compensate more effectively for its existence (such as a hearing aid, participation in speech therapy, or use of a communication device). Such information might enable better counseling of individuals and their families about the importance of addressing a communication disorder and also enable advocacy to third-party payers about the importance of funding these types of interventions. 


\section{Literature Review}

\section{Communication and Aging}

Communication forms the foundation of social interaction (Heine \& Browning, 2002). Successful interpersonal communication has been defined the "successful joint establishment of meaning" that occurs as a result of "a two-way process (expressive and receptive) in which messages are negotiated until the information is correctly understood by both parties" (Joint Commission, 2010, p.1). It is a process that, under normal circumstances, occurs without effort but a disruption of any of these processes can cause profound alterations. Further, communication occurs not just in an interpersonal context but also at a distance, such as by telephone, letter, and electronic forms of communication. The ability to hear, read and understand language is essential to making sense of the world around us, such as by watching TV, by reading a newspaper, or by listening to the radio. Communication in all its many forms is implicit to most activities, including those associated with activities of daily living, educational and vocational performance, social roles and functions, and the ability to participate in community and civic life. The importance of communication for health is reinforced by the fact that it is one of the key domains in the World Health Organization's conceptual model of health and disability (2001). In healthcare, communication is now considered a patient's right and an essential component of quality care and patient safety (Joint Commission, 2010). So central has communication become that, in an era of information technology, the health and economic problems faced by those with difficulty communicating has been described as "the survival of the fittest for the 21 st century" (Ruben, 2000). 
In older adults, communication is central to the process of successfully adjusting and adapting to the aging process (Lubinski \& Welland, 1997). Studies of communication in normal aging have shown that the conversational skills of normally aging older adults tend to remain well-preserved (Shadden, 1997). With increasing age, the prevalence of conditions that may interfere with communication also increases. These medical conditions include dementia, stroke, cancer of the head and neck, traumatic brain injury, and progressive neurological diseases such as Parkinson's Disease or Amyotrophic Lateral Sclerosis (ALS). As this list suggests, the conditions that cause communication impairments vary widely in their type and severity as well as in their co-occurrence with other types of disability and functional limitations (Yorkston et al., 2010). The terms "communication disability" and "communication disorders" include a wide variety of disorders related to speech, language, hearing and cognitive function that may interfere with the process of communication. ${ }^{1}$ Nonetheless, research into the experiences of individuals with communication disorders of varying etiologies and types demonstrates many commonalities (Baylor, Burns, Eadie, Britton, \& Yorkston, 2011).

In the subsequent sections, the literature will be reviewed with regard to communication changes associated with normal aging, prevalence data relating to

\footnotetext{
${ }^{1}$ The terms "communication disorder," "communication disability," and "communication impairment" are largely synonymous and used interchangeably in the literature. Although the term "communication disorder" is the more widely used in this area of research and is used to describe the field as a whole (as in the "National Institute on Deafness and Other Communication Disorders" or NIDCD, for example), the more neutral term "communication impairment" has been used more often. As discussed below, changes in communication occur in association with normal aging. These tend to be less severe and less disruptive than those associated with pathological conditions and therefore may not be considered "disordered" per se. Communication impairments may be conceptualized as existing on a continuum, from those which are negligible or relatively mild and are associated with typical aging to those which are associated with a disease process and may be more severe. The assumption of the current study is that even changes associated with normal aging may have an impact on social relationships and other outcomes of interest. The purpose of the current study is to examine the impact of the severity of communication impairment, regardless of etiology, type, or categorization (i.e. normal/typical or pathologic). Consequently the more neutral term "communication impairment" has generally been used throughout.
} 
communication disorders, findings from descriptive studies regarding their impact, and theoretical frameworks for understanding the process by which handicap, disability, and adaptation occur.

Communication and normal aging. A number of studies have been published documenting that, even in the absence of pathology, the communicative abilities of older adults change over time. These changes may have an impact of a wide variety of activities of daily life including those involving communication (Farley, McLafferty, \& Hendry, 2006). Worrall and Hickson (2003) reviewed the research with regard to the various different aspects of communication, including hearing, voice, speech, language, and conversational discourse. Typically, hearing changes result in a decreased sensitivity to higher frequencies that can result in a reduced ability to understand speech, particularly in adverse listening conditions (e.g. against background noise or over a poor telephone connection). Voice and speech changes include reduced respiratory support for speech, less precise articulation (particularly with poor or missing dentition), a slower rate of speech, changes in vocal pitch (higher for men and lower for women), and poorer vocal quality. These changes, in combination, can result in decreased intelligibility, reduced vocal loudness, and negative perceptual changes. The voice may be perceived as weak, hoarse, or gender-inappropriate (i.e. more feminine in men and more masculine in women). Changes also occur in linguistic processing, including reduced speed and accuracy of word-retrieval, as well as greater difficulty with comprehension of linguistically complex or technical communication. In conversation, such changes can result in difficulty understanding complex and lengthy discourse, decreased efficiency of expression, and greater ambiguity. The structure of conversation also changes over time, 
with verbal expression characterized by decreased cohesion, decreased rate, more words per clause, and an increased degree of topic maintenance. Nonetheless, studies of communication in normal aging have shown that the conversational skills of normally aging older adults tend to remain well-preserved, even though the semantic content and syntactic structure of language use change over the life-course (Shadden, 1997).

In addition, it is important to consider the types of social and communicative activities in which older adults engage. Horgas, Wilms, and Baltes (1998) used data from the Berlin Aging Study to investigate the everyday activities of community-dwelling older adults. Their data show that most of the day was spent engaged in instrumental activities of daily living (e.g. shopping, household chores), personal care (e.g. getting up, eating, preparing for bed), leisure activities, watching TV, and resting. On average more than ten hours a day was spent alone, while about three hours were spent with a spouse or partner. Other people that the older adults had contact with included roommates, groups, children, relatives, friends, and paid caregivers. Studies of the naturalistic communication of older adults suggest considerable variability with regard to a number of factors, such as the amount of time spent interacting with other people, the number of people involved, and the nature and topics of those interactions (Davidson, Worrall, \& Hickson, 1998; Shadden, 1988).

The prevalence of communication impairments in older adults. About one in six Americans has a communication disability of some kind that may have a significant impact on education, employment and quality of life (NIDCD, 2006). Communication disability includes a variety of disorders related to speech, language, hearing and cognitive function for communication. Hearing impairment is the most prevalent 
communication disorder nationally and occurs in around $15 \%$ of all adults. The prevalence of hearing impairment increases with age and it is the third most common chronic condition of older adults (Wallhagen, 2002). Estimates vary but, according to one study using nationally representative data, hearing loss occurs in $45 \%$ of those aged 60 $69,68 \%$ of those aged $70-79$ and $89 \%$ of those aged 80 or more (Lin et al., 2010).

It is difficult to estimate the national prevalence of other types of communication impairments because population-based studies do not collect this type of information routinely. Consequently, most estimates of prevalence are based on projections using data from specific populations (American Speech-Language-Hearing Association [ASHA], 2008). In one of the largest surveys of older adults, data were used from 12,000 Medicare beneficiaries aged 65 or more who had been surveyed as part of the Medicare Current Beneficiary Survey (Hoffman et al., 2005). Although communication impairments were not formally assessed in this study, individuals were asked about a variety of problems, including difficulties with communication. Forty-two percent of the sample reported hearing problems, $26 \%$ had writing problems, and $7 \%$ had problems using the telephone. Based on these data, it was estimated that $55 \%$ of all Medicare beneficiaries (more than 16 million) had some type of communication disability. Approximately $1 \%$ had a severe communication disability in all three areas of hearing, writing, and using the telephone. Greater communication disability was associated with a number of sociodemographic variables, including advanced age, poorer health, greater physical impairment, lower SES, less education, male gender, rural residence, and being an unmarried individual coresiding with others (suggesting some degree of functional dependency and a reduced ability to live independently). In studies of older adults in long-term care and healthcare 
settings, the prevalence of communication disability may be even higher. In a study of medical inpatients, nearly $16 \%$ of admissions to university hospitals involved patients with one or more disabilities severe enough to prevent almost any form of communication (Ebert \& Heckerling, 1998). A review of data from more than 18,000 residents of nursing homes indicated that more than 33\% had "inadequate communication," indicating either that they were unable to effectively make themselves understood (Resnick, Fries \& Verbrugge, 1997). These data suggest that communication impairments in older adults exist on a broad continuum (Yorkston et al., 2010) from those who are relatively healthy and experience relatively little disruption in their ability to communicate with others to those who experience more severe communication impairments of various types. Kathryn Yorkston and colleagues (2010) also distinguish two separate groups. The "disability with aging group" includes those who spend most of their lives without a disability and experience either age-related changes (such as hearing impairment) or a new-onset medical condition (such as a stroke or Parkinson's Disease) that tends to occur in later adulthood. In contrast, the "aging with disability group" includes those who have had a lifelong disability which may be exacerbated by agerelated changes (such as cerebral palsy or multiple sclerosis). The presence of a communication disorder early in life may significantly impact the development of normal social relationships over the life-course with consequences in older adulthood, as has been found in older adults with cerebral palsy or a history of stuttering (Ballin \& Balandin, 2007; Bricker-Katz, Lincoln, \& McCabe, 2009).

The impact of communication impairments on the lives of older adults.

Communication impairments are associated with increased risk of depression, social 
isolation, interpersonal strain, loneliness, and poorer quality of life (Yorkston et al., 2010). These findings have been confirmed in a variety of clinical populations, including older adults with uncorrected hearing loss (Arlinger, 2003), aphasia following a stroke (Davidson et al., 2008), Parkinson's Disease (Carter et al., 1998), Amyotrophic Lateral Sclerosis (Joubert, Bornman, \& Alant, 2011), multiple sclerosis (Bringfelt et al., 2006) cerebral palsy (Ballin \& Balandin, 2007), neurological speech impairments (Walshe \& Miller, 2011), head and neck cancer (Palmer \& Graham, 2004), voice disorders (Siupsinskiene, Razbadauskas, \& Dubosas, 2011), and stuttering (Bricker-Katz et al., 2009). In spite of these findings, it is difficult to estimate the impact of a communication disorder on psychosocial health and quality of life because they often co-occur with other health problems and functional limitations. Interviews with individuals with disabling conditions that cause physical as well as communication impairments, for example, emphasize that particular condition must be considered globally and that communication is just one "part of the picture" (Walshe \& Miller, 2011, p.198).

To date, most studies in the literature have focused on a single diagnosis or disorder. In one of the few studies that have gathered information about the impact of communication disorders due to a wide range of etiologies, there were many commonalities among quite different types of impairments (Baylor et al., 2011). Participants described having to use alternate methods of communication, adapt their method of communication, rely on others to communicate for them, and/or ask for accommodations from their communication partners. In some situations, these strategies or accommodations were not effective and this resulted in withdrawal from a variety of social situations well as life roles and positions of responsibility, including those related 
to work, group membership, and community involvement. They also described feelings of isolation and marginalization, of feeling "like a bystander," "out of the loop," or "ignored," as well as feeling as though they had lost their "sense of self" (pp.275-276).

Communication impairments and mental health. Communication impairments are associated with a wide variety of negative mental health outcomes, including poorer emotional well-being and quality of life, mood disorders, and increased levels of distress, anxiety and depression (NIDCD, 2006). Hearing loss is associated with a higher incidence of depression and depressive symptoms across the life-course and, in addition, those with greater communication problems secondary to a hearing impairment have the highest levels of mental distress (Arlinger, 2003; de Graaf \& Bijl, 2002; Jones \& White, 1990). Similar findings have been found with other types of communication impairments. The presence and severity of a voice problem has been found to be associated with higher levels of anxiety, depression, and psychological distress (Deary, Wilson, Carding \& Mackenzie, 2003; Siupsinskiene et al., 2011). Evidence for a causal link between communication impairments and poorer mental health is reinforced by studies that have shown improvements in psychological outcomes after a wide range of interventions (Baylor, Yorkston, Eadie, \& Maronian, 2007; Boi et al., 2012; Hawkins, 2005; Heydebrand, Mauze, Tye-Murray, Binzer \& Skinner, 2005; Liu et al., 1998; Mulrow, Aguilar, Endicott, Tuley, et al., 1990; Murry, Cannito, \& Woodson, 1994).

The impact of "sensory impairment" (which includes both vision and hearing impairments) has been investigated using large-scale, population-based data from a number of countries. After controlling for a range of health and demographic characteristics, sensory impairments have been found to be significant predictors of a 
wide variety of psychosocial outcomes including loneliness, depression, suicidal ideation, self-perceived handicap, quality of life, and life satisfaction (Blay, Andreoli, Fillenbaum, \& Gastal, 2007; Bourque, Leger, Pushkar, \& Beland, 2007; Capella-McDonnall, 2005; Chou \& Chi, 1999; Dugan \& Kivett, 1994; Yip et al., 2003). Some studies have found that hearing impairment is a less powerful predictor of depression than visual impairment or other predictors (Capella-McDonnall, 2005; Chou, 2008; Mulrow, Aguilar, Endicott, Velez, et al., 1990). This is not true of all studies, however. In a study by Johan Ormel and colleagues (1997) both hearing and visual impairment were found to be two of the strongest independent predictors of psychological distress.

Communication impairments and disability. Both vision and hearing impairments have been found to be independently associated with declines in everyday activity patterns. Using data from the Berlin Aging Study, Marsiske, Klumb, and Baltes (1997) examined the relationship between visual and auditory acuity on two measures of everyday activity, namely perceived competence with basic activities of daily living and the amount of participation in discretionary social and leisure tasks. Hearing and vision were significant independent predictors of both outcomes and could explain most of the age-related variance in everyday activities.

Sensory impairments have been shown to have a significant negative relationship with measures such as functional disability, health, risk of falls, and mortality but, again, some researchers have found hearing loss to be a less-powerful predictor than visual impairment (Horowitz, Brennan, \& Su, 2001). It is possible that hearing impairment may interact with other types of disability. For example, there is some evidence that hearing impairment alone may be less significant than hearing impairment combined with visual 
impairment, which has caused some authors to identify "dual sensory impairment" as the stronger predictor of negative outcomes (Berry, Mascia, \& Steinman, 2004; Brennan, Horowitz, \& Su, 2005; Brennan, Su \& Horowitz, 2006; Harada et al., 2008; Saunders \& Echt, 2007). These findings suggest that it may be important to consider the combined effect of different types of disabilities, as this may result in a cumulative disadvantage with regard to a wide range of important outcomes.

Evidence for an interaction between different types of disability is provided by a study in which the relative impact of physical and sensory impairments was examined (Kempen, Verbrugge, Merrill, \& Ormel, 1998). Using data from a community-based sample of older adults, the authors found that vision and hearing impairments had independent and unique predictive value with regard to different types of limitations. Vision loss was associated with poorer role function, while hearing loss was associated with poorer social function. In addition, to these main effects, vision and hearing losses exacerbated the impact of other types of impairments on disability measures, supporting the hypothesis that sensory impairments might interact with physical limitations.

Communication impairments and quality of life. Quality of life can be defined in a number of ways. According to one definition, individuals judge their quality of life based on the gap between their expectations for their life and their perception of their current circumstances (Calman, 1987). The concept of quality of life is typically described as being both subjective and multidimensional. Quality of life has also been shown to change over time as a result of both internal forces (e.g., changes in perceptions or goals) and external forces (e.g., changes in life circumstances). A strong association has been reported between communication and quality of life in a wide variety of 
populations (Dalton et al., 2003; Magilvy, 1985; Mulrow, Aguilar, Endicott, Velez, et al., 1990; Palmer \& Graham, 2004). The possibility of a causal relationship is supported by improvements in quality of life after interventions for a wide range of different disorders and conditions (Berg, Hapner, Klein \& Johns, 2008; Brooks, Hallam, \& Mellor, 2001; Carozza \& Shafi, 2013; Mulrow, Aguilar, Endicott, Tuley, et al., 1990; Vingerhoets et al., 1999).

Communication impairments, benefit finding and growth. Most research regarding the psychological function of individuals with disabilities has concentrated on negative outcomes, such as depression, anxiety, or posttraumatic stress (Bombardier, Ehde, Stoelb, \& Molton, 2010). In recent years researchers have been increasingly interested in the positive shifts in behaviors, beliefs, and emotions that some people with disability may experience. In the health and trauma literatures, these phenomena have been referred to as "benefit-finding" or "post-traumatic growth." The types of benefit or growth most commonly described can be grouped into three categories: those that emphasize enhanced personal relationships (e.g. increased closeness with friends/family, more compassion for others); those that describe a changed view of oneself (e.g. an increased awareness of one's strength/vulnerability); and a change in life philosophy (e.g. increased appreciation for every day).

As with research on other kinds of disabilities, most research to date has concentrated on the negative implications of communication impairments on psychosocial function. There are, however, a few studies which mention some positive aspects consistent with the literature on benefit-finding and growth. Individuals treated for head and neck cancer, for example, have reported the following: increased feelings of 
social support from family, friends, healthcare providers, and other patients; feelings of being lucky at having survived; and having a new appreciation and perspective on life (Fletcher et al., 2012). Older adults with hearing impairments have reported that they are able to pursue certain activities in tranquility and be more focused, introspective, and creative by deliberately not using their hearing aids at times (Lockey, Jennings, \& Shaw, 2010). Others describe using a hearing-impairment for personal advantage, such as avoiding unwanted situations (Stephens \& Kerr, 2003). Some individuals also report a sense of pride at learning to be able to communicate successfully despite the presence of a communication disorder (Baylor et al., 2011; Stephens \& Kerr, 2003). This would be consistent with the literature on positive growth and benefit-finding. It is possible that individuals with communication impairments may perceive some positive aspects. Based on the previous literature, positive aspects might include an increased appreciation of things that had been taken for granted, increased closeness and social support from friends and family, a sense of fulfilment from pursuit of personal pursuits, or a sense of accomplishment from overcoming challenges and obstacles that they experience as a result of their communication difficulties. It is also possible that changes in social relationships, such as a smaller but emotionally closer social network, might be perceived as positive and desirable by those with a communication impairment. Once again, it is possible that "bigger is not always better" with regard to social network size (Bowling, 1994, p.42). 


\section{Theoretical Frameworks for Understanding the Impact of Communication}

\section{Impairment}

Models of health and disability. A number of theories have been developed to understand the process of becoming disabled (Putnam, 2002). One of the most commonly cited theories is Nagi's Disablement Model (1965), positing that a disease process or injury causes an active pathology which, in turn, leads to an impairment at the level of a bodily function. This impairment then results in a functional limitation which, in turn, causes physical or psychological restrictions in the social context (i.e. a disability) and limitations in the performance of normal roles (i.e. a handicap). This model is illustrated below (Figure 1a.) and has formed the basis for other conceptual frameworks, including the Institute of Medicine's "enabling-disabling process" and the World Health Organization's (WHO) original International Classification of Impairments, Diseases and Handicaps (ICIDH; 1980) model. This model can be applied to the field of communication disorders. For example, prolonged exposure to noise may cause damage to the hair cells in the inner ear (disease/disorder) resulting in a moderate hearing impairment (impairment). This impairment may lead to difficulty understanding speech in noisy situations and talking on the telephone (disability) and, in turn, moderate participation restrictions, including withdrawal from many social situations and volunteer activities (handicap). One of the limitations of the Disablement and similar models, however, is that they assume a medical cause for all disability and that the association is necessarily linear from disease through to handicap without considering the role of the social environment or individual factors (e.g., adaptation). Enderby and John (1997) and Worrall and Hickson (2003) provide a number of examples of the inadequacies of this 
model. With regard to a hearing impairment, it is possible that a moderate impairment may result in a moderate disability, as in the example listed above. It is possible, however, that a severe impairment may cause minimal disability. For example, a woman with a severe bilateral sensorineural hearing loss (impairment) may use a wide range of adaptive equipment (including a conference microphone, FM system, flashing ringer on the telephone, adaptive telephone with telecoil and volume control, closed captioning, email and texting) to result in mild handicap and minimal disability (she has a busy social life and works full-time for a disability agency). Clearly the relationship between disease and disability is not strictly linear, as depicted in the medical model of disability.

The limitations of this type of framework are also demonstrated by the relation between objective measures of function and subjective assessments of handicap. By way of an illustration, the severity of a hearing impairment can be measured in a number of different ways. Most commonly, objective testing is accomplished by use of standardized tests in which the thresholds at which pure tones are heard by an individual at different levels of intensity (pure tone audiometry; ASHA, 2005). Other tests involve the ability to identify individual words to determine the threshold at which speech is detectable (ASHA, 1988). However, this does not assess the individual's perception of their own hearing impairment and its impact on their daily life, function, and emotional well-being. To do so, a measure of "hearing handicap," such as the Hearing Handicap Inventory for the Elderly Screening Version (HHIE-S; Ventry \& Weinstein, 1983) must be used (ASHA, 1997). With regard to the literature on hearing impairment, considerable variability has been reported in the association between subjective and objective measures (Wallhagen, 2002). In some studies objective measures have been found to be 
strongly associated with subjective handicap measures (Rudberg, Furner, Dunn, \& Cassel, 1993; Weinstein \& Ventry, 1982), but other studies have found very weak relationships (Brainerd \& Frankel, 1985). Further, even mild impairments (measured objectively) have been shown to have a significant negative impact on psychosocial function (Mulrow, Aguilar, Endicott, Velez, et al., 1990; Scherer \& Frisina, 1998). Clearly, the relationship from impairment to handicap is not linear, as suggested by the medical model of disability, but is affected by a range of other individual and contextual factors.

To better explain the relationship between health and disability, "biopsychosocial" models of disability have increasingly gained currency (Eadie, 2003; Putnam, 2002). These models place a different emphasis on the interaction between the individual and the environment in the transition towards a disability. In the International Classification of Functioning, Disability and Health (ICF) model, the most recent the revision of its disability framework, the WHO (2001) has moved away from a "medical model" of disability (Figure 1a.) towards a "biopsychosocial" model of health (Figure 1b.). This takes into account not only the contribution of biological factors but also psychological and social factors. An individual's health and health-related domains are described from the perspective of the body, the individual, and society in the following classifications: 1) body functions, which includes the physiological functions of a body systems, including psychological functions; 2) body structures, which include the anatomical parts of the body; and, 3) activities and participation, which include actions by individuals themselves and the relationship of these actions to the rest of the individual's life. There are also contextual factors, which include both environmental 
factors and personal factors. Environmental factors are those which are generally outside the individual's control, such as the social and political environment, legal context, cultural beliefs, and the attitudes of others. Personal factors include individual characteristics independent of a particular health condition, such as race, gender, age, educational level, coping styles, upbringing, personality traits, and lifestyle. Personal factors are not coded in the ICF because of their wide variability among individuals and cultures, but they are anticipated to have an impact on function as well as on the outcomes of different interventions (WHO, 2001).

(a)

Disease

or $\longrightarrow$ Impairment $\longrightarrow$ Disability $\longrightarrow$ Handicap

Disorder

(b)

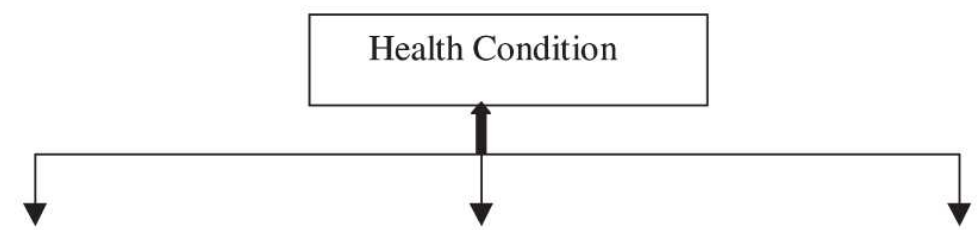

Body Functions or Structures

Individual Activity

Societal Participation

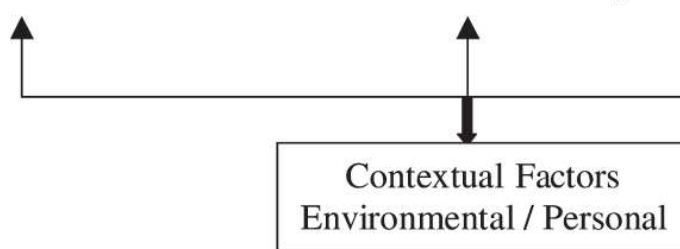

Figure 1. Differences between a medical model (a) and a biopsychosocial model (b) of health and disability. Adapted from "The ICF: A Proposed Framework for Comprehensive Rehabilitation of Individuals who use Alaryngeal Speech," by T.L. Eadie, 2003, American Journal of Speech-Language Pathology, 12, pp.190-1. 
Communication functions are represented at all levels of the ICF, including body function, body structures, and activity and participation. Communication is one of the nine domains at the level of both activity and participation and, in addition, communication is implicit to many of the other types of activities and functions included in the model, including "learning and applying knowledge," "interpersonal interactions and relationships," "major life areas," and "community, social and civic life." The relevance of communication to so many aspects of the model clearly reinforces the notion that communication is central to many of the everyday activities of life (Worrall \& Hickson, 2003).

According to Threats (2006), the ICF has many potential advantages for understanding communication disorders, including its utility as a framework for studying the epidemiology of communication disability as well as its application for guiding clinical practice, social policy, education, and research. Threats argues that using the ICF to guide research could fill a number of important needs in the field, including the relationship between body function/body structure and activity/participation behaviors, an increased focus on the effects of the ICF constructs on quality of life, and an increased focus on the role of personal factors in the process of rehabilitation and adaptation. 


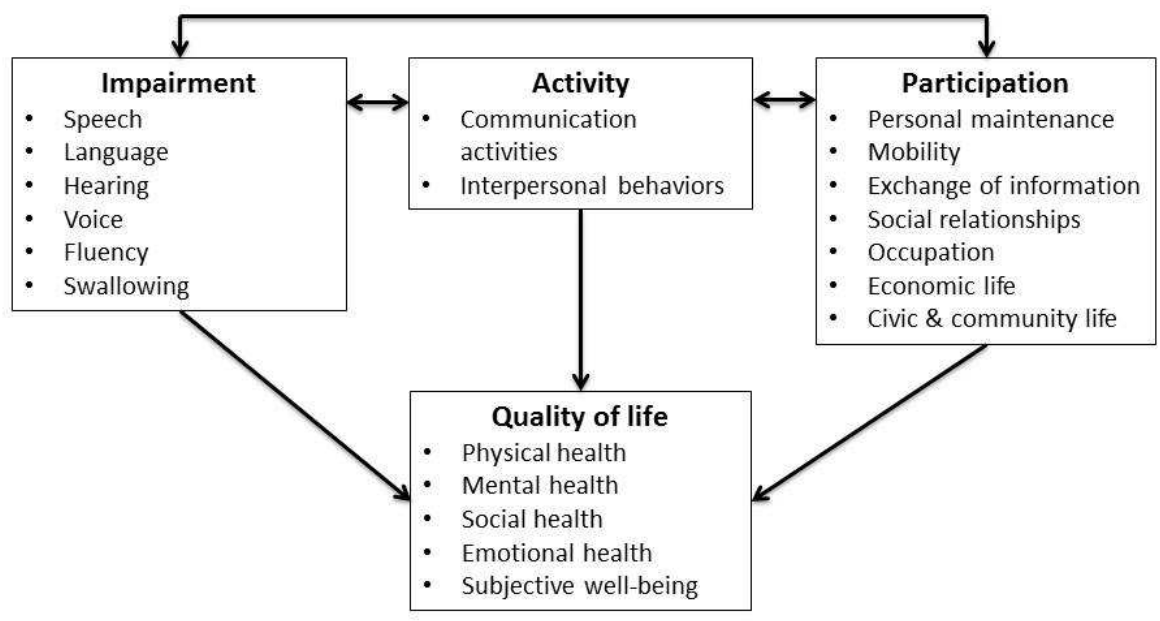

Figure 2. A conceptual model of the relationship between communication and quality of life. Adapted from Communication Disability in Aging: Prevention to Intervention, by L. Worrall and L. Hickson, 2003, Mason, OH: Cengage Learning, p.25.

Concepts such as quality of life, mental health, and well-being are not part of the ICF model but can be hypothesized to be associated with all of the ICF levels. For example, Figure 2 depicts a theoretical model of the relationship between communication and quality of life, using the ICF framework (Cruice, Worrall, \& Hickson, 2000). This conceptual model is one of the few in the literature to use the ICF framework to explicitly model the relationship between the various levels of communication and social and psychological well-being.

\section{The role of personal factors and individual differences in adapting to}

communication impairments. Personal factors are not coded in the ICF because of their wide variability among individuals and cultures but they are anticipated to have an impact on function as well as on the outcomes of different interventions (WHO, 2001).

Nonetheless, considerable interest exists with regard to determining the factors which promote greater adaptation, particularly in the case of reduced physical abilities or 
decline. Adaptation refers to a range of behaviors, such as coping, goal-setting, problemsolving and other efforts on the part of the individual to maintain psychological wellbeing (Ruth \& Coleman, 1996). Given the interactive nature of the disablement process, a number of psychosocial theories have been used to examine why some individuals are able to adjust and adapt to disability well while others do so more poorly.

A number of theories have potential utility with regard to the study of older adults with communication impairments although, to date, none of them has been used widely in the research relating to communication disorders. Three theories concentrate on changes made at the individual level in the process of adaptation, namely: a) models of stress and coping; b) Selective Optimization with Compensation; and, c) theories of selfefficacy, control, and mastery. In addition to theories that consider factors at the individual level, it is also worth considering two other theories that place communication in an environmental context. Communication Accommodation Theory (Giles, Coupland, \& Coupland, 1991; McIntosh, 1996) views the process of communication from a dyadic perspective in terms of the relative contribution of accommodations and adjustments made by both partners in a communicative exchange. Person-Environment Fit (Lawton 1982, 1987) examines changes both at an individual and a contextual level. These theories are described in greater detail below. Other theories that could be of relevance were excluded, such as theories of dyadic or family coping, and Family Systems theory, due to the rarity of their use in communication research or their conceptual overlap with the other models.

Models of stress and coping. Stress is a broad concept that can be applied to a wide-range of social phenomena and it is also a pathway for negative outcomes through 
its association with physical health (Yancura \& Aldwin, 2008). Stress is a useful approach for the study of health because levels of physiologic stress (e.g., blood pressure, heart rate or stress-related hormone levels) can be a predictor of subsequent disease (e.g., cardiovascular disease). Alternatively, health problems can also be viewed as a source of stress and therefore one of a number of stressors (Holmes \& Rahe, 1967). For individuals with communication impairments, both the underlying medical condition (e.g., a stroke) and the associated communication impairment may be regarded as potential stressors.

Stressors are demands made by the internal or external environment that cause a disruption in the individual's equilibrium and require effort on the part of the individual to maintain physical and psychological well-being. Coping refers to the many cognitive, behavioral, and emotional ways that people deal with stressful situations to minimize the impact of stressful situations and to optimize physical and mental health (Taylor \& Stanton, 2007). Coping strategies have been grouped into avoidant strategies and problem-focused strategies (Aldwin \& Gilmer, 2004). Avoidant coping strategies have typically been associated with worse health outcomes, while those with more problemfocused approaches tend to live longer and have better symptom-management and disease-control (Connor-Smith \& Flachsbart, 2007; Penley, Tomaka, \& Wiebe, 2002). It is important to note that coping is not a reaction at a single time-point but a dynamic process in which multiple reactions may occur over time (Folkman \& Moskovitz, 2004). In addition, coping style is not necessarily a static component of personality. Individuals appear to cope in different ways depending on the context and stressors and, consequently, the same coping pattern may be viewed as either adaptive or maladaptive depending on the context (Folkman, Lazarus, Dunkel-Schetter, DeLongis, \& Gruen, 
1986). Despite the ample evidence that coping is relevant to the study of aging and health, many contradictions are apparent in the literature (Yancura \& Aldwin, 2008). These contradictions suggest that the study of coping is complex and findings may vary significantly depending upon how coping is conceptualized and assessed. In addition, despite the wealth of literature on the topic, there is little consensus about how to conceptualize or measure coping (Skinner, Edge, Altman, \& Sherwood, 2003).

To date, relatively few studies have examined the role of coping in outcomes of individuals with communication disorders. Several studies of survivors of laryngeal cancer (Blood, Luther, \& Stemple, 1992; Blood, et al., 1994; Eadie \& Bowker, 2012), have found strong associations between communication, coping style, self-esteem, and well-being. Similar findings have been found in adults with hearing impairments (Gomez \& Madey, 2001). Because these studies were cross-sectional, however, it is difficult to interpret whether differences in coping style were the cause or the effect of differences in communication or of other factors. Intervention studies have reported short-term benefits from programs to teach coping skills to individuals with hearing impairment (Wallhagen, 2002), but evidence from large samples with long-term outcomes is currently lacking. The utility of the coping framework is that it considers a number of variables that seem to be important in measuring treatment outcomes. In particular, the coping perspective recognizes that individuals have agency and have some degree of control in shaping their reaction to stressful circumstances in terms of strategies that they may choose to employ.

Selective Optimization with Compensation. In the context of aging, the theory of Selective Optimization with Compensation has been developed to describe how some individuals manage to successfully adapt to the inevitable losses caused by the aging 
process, positing three factors that can be used in combination (Baltes, 1987, 1997). In a classic illustration, the concert pianist Arthur Rubinstein was asked how he was able to continue performing professionally as he got older and responded that he played fewer pieces of music (selection), practiced more before a concert (optimization), and played the slow sections more slowly so that the fast sections would seem faster than they actually were by comparison (compensation; Baltes \& Carstensen, 1999). Selective Optimization with Compensation has been used to explain the apparent paradox that many older adults are able to maintain high levels of function, despite physiological changes, with regard to a wide range of activities (e.g., Charness, 2000; Salthouse, 1990). This framework also helps to explain changes in resource allocation across the life-course (Baltes, 1997). In childhood, the primary focus of the individual is directed toward growth; during adulthood, the predominant focus is toward maintenance and resilience. As individuals age, more and more resources are directed toward regulation or management of loss to maintain adequate function (Baltes \& Baltes, 1990; Baltes \& Carstensen, 1996; Dixon \& Backman, 1995; Marsiske, Lang, Baltes, \& Baltes, 1995). Although this model has not been used extensively in the area of communication disorders research, the concepts of goal setting (selection) and adjustment (compensation) are fundamental to most models of rehabilitation (Hoenig, Nusbaum, \& Brummel-Smith, 1997).

Theories of self-efficacy, control, and mastery. Control beliefs were first studied as part of social learning theory (Rotter, 1966) using the term "locus of control." The concept of locus of control is a measure of the extent to which individuals believed that the events in their own lives were under their influence. Early conceptualizations 
emphasized perceived sources of control as being either internal (i.e., being due to effort, ability, skill, intelligence) or external (i.e., due to luck, fate, the influence of others). Internal locus of control has consistently been shown to be strongly associated with better health outcomes across a wide array of studies (Wallston, Wallston, Smith, \& Dobbins, 1987). In its original conception, control was conceptualized as a general, stable characteristic of the individual personality that would generalize across domains. Since then the concept of control has been studied in a number of variations in the health and psychology literature, using terms such as personal mastery, locus of control, learned helplessness, and self-efficacy, all of which relate to an individual's ability or perceived ability to produce desired outcomes (Lachman, Neupert, \& Agrigoroaei, 2011). Recent research has demonstrated that control beliefs vary over time, across different types of activity, and also across the life-course, increasing from early adulthood until reaching a peak in middle age, followed by a subsequent decline (Eizenmann, Nesselroade, Featherman, \& Rowe, 1997; Lachman, 1986; Lachman, Rosnick, \& Rocke, 2009; Lefcourt, 1984; Mirowsky \& Ross, 2007). In recent years, the concept of self-efficacy has been widely used in the health literature. As conceptualized by Bandura (1977), selfefficacy is the conviction that one can successfully execute the behavior required to produce the outcomes. Since its formulation, the concept of self-efficacy has been applied to a wide range of issues, from managing health problems such as diabetes, arthritis, obesity, visual impairment, and cancer, to optimizing lifestyle and personal behaviors with regard to exercise, diet and educational achievement (Romppel et al., 2013). Bandura's Social Cognitive Theory and the self-efficacy framework has been demonstrated to be a model for explaining and mediating dynamic behavior change, 
including behaviors related to managing chronic health conditions and promoting healthy lifestyles (Bandura, 1977, 1986, 1997; Shortridge-Baggett, 2001). Self-efficacy perceptions are domain-specific, indicating an individual may have high self-efficacy for the skills associated with one type of activity but low self-efficacy for other domains of activity. Self-efficacy is not a fixed personality construct but changes as a result of a wide variety of life experience and can be influence by mastery experiences, vicarious experience, verbal persuasion and physiological and affective states.

To date, the concept of self-efficacy has been infrequently applied in the fields of speech pathology and audiology, although it has had some limited use in audiologic rehabilitation, stuttering, and voice disorders (Bonilha \& Dawson, 2012; Finn, 2003; Gillespie \& Abbott, 2011; Smith \& West, 2006; van Leer, Hapner, \& Connor, 2008). Self-efficacy has been hypothesized to be one of the factors influencing global outcomes in models of rehabilitation after intervention for a communication disorder in older adults (e.g. Clark et al., 2012). Self-efficacy is also similar to other constructs that have been hypothesized in the literature. For example, Babbitt and Cherney (2010) have proposed a related concept, namely "communication confidence" based on the self-reported experiences of individuals with aphasia. They hypothesize that confidence in the ability to communicate may be strongly associated with the constructs of personal autonomy, self-efficacy, and self-determination. When an individual's interactions with others prove problematic due to the presence of a communication impairment, this may lead to a decreased confidence in the ability to communicate personal wishes, diminished autonomy, and result in learned helplessness. Consequently feelings of control with regard to communication and the ability to interact across a wide variety of social settings 
may be associated with more global feelings of control, competence, or dependency. This is consistent with other research that has shown communication impairments to be associated with a diminished sense of personal competence, increased reliance on others, and a loss of the sense of self (Baylor et al., 2011; Marsiske et al., 1997).

Communication Accommodation Theory. Communication Accommodation Theory (Giles, Coupland, \& Coupland, 1991; McIntosh, 1996) is taken from the field of communication research and has been applied to a wide variety of different types of exchanges. Communication Accommodation Theory is based upon the premise that, in all interactions, speakers and listeners adjust to each other's patterns of communication. This includes changes in choices of style of speech (e.g. accent), word choice, rate of speech, politeness, pitch and other characteristics, depending on the context, the relationship between the individuals, and the nature of the interaction. This theory has previously been applied to the study of communication and aging (Ryan, Giles, Bertolucci, \& Henwood, 1986). Satisfying and effective communication is the result of both participants making appropriate accommodations. Inappropriate accommodations can take two different forms. Underaccommodation can occur if one of the participants has failed to adjust his or her method of communication appropriately (e.g. the speaker fails to recognize that they are speaking too quietly to be understood and does not modify the loudness of their speech). Overaccommodation can also occur when a speaker (possibly as a result of stereotypical views of aging adults) makes more changes to their communication than is necessary, possibly speaking louder than necessary or adopting a patronizing or demeaning pattern of language (e.g. "elderspeak"; Kemper, 1994). 
Although Communication Accommodation Theory has not been widely utilized in the communication disorders literature, it can easily be applied to this field (Worrall \& Hickson, 2003). The individual with a communication impairment may compensate more or less effectively for the presence of an impairment. In addition the reactions of the communication partner may also affect the success of the interaction. Examples of underaccommodation by the communication partner would include not adjusting their communication in order to be adequately understood, such as not speaking loudly enough to be heard over background noise, speaking too quickly, or using more complex vocabulary and grammar than can easily be understood. Examples of overaccommodation would include interrupting, completing sentences on the part of the speaker, speaking over-loudly, oversimplifying the content of the conversation, treating the partner as though they are more impaired than they actually are, talking to a spouse or partner instead, or avoiding communication altogether. These types of interactions are commonly reported by individuals with communication impairments (Baylor et al., 2011; Hétu et al., 1993). In addition, the fact that friends and family members of individuals with communication impairments make considerable adaptations in order to maintain their relationship over time is consistent with this model (Baxter, Braithwaite, Golish, \& Olson, 2002; Bute, Donovan-Kicken, \& Martins, 2007; Kemper, Lyons, \& Anagnopoulos, 1995). The advantage of Communication Accommodation Theory is that it acknowledges that actions on the part of the individual may or may not be effective in compensating for a communication impairment, depending on the social context. It also considers the individual within their cultural context in terms of how older adults with communication impairments are viewed and treated. 
Person-Environment Fit. Another widely-used framework within social gerontology is that of Person-Environment Fit (or "P-E Fit"). Based on the work of Lewin $(1938,1951)$, the model considers any event to be the result of an interaction between the individual and his or her environment (Lawton, 1982, 1987). The environment is not a static backdrop but affects the behavior of the individual and can, in turn, be shaped and controlled to varying degrees to achieve congruence (Parmelee \& Lawton, 1990). Competence is neither a function solely of the individual, nor of the environment. Competent behaviors occur when the abilities of the individual match the demands and resources of the environment. Consequently, when a change occurs, either at the level of the individual or the environment, adaptation can be seen as a dual process in which the individual attempts to maintain equilibrium by reacting to environmental cues and many, in turn, shape that environment. Optimal well-being is achieved when an individual's needs are in equilibrium with environmental characteristics (i.e. there is “congruence” or "good P-E fit"; Kahana, 1982). For older adults who experience functional decline, adaptive changes to the environment can be made to promote better function. If these types of changes are not made, however, the individual will experience poorer levels of functioning and adaptation (Golant, 2003; Iwarsson, 2005).

With regard to communication disorders, the concept of P-E Fit has been used to describe the "process of disablement" such as that experienced by community-dwelling older adults with hearing impairment (Iwarsson, 2005; Verbrugge \& Jette, 2004). A variation of P-E Fit, Roy’s Adaptation Model (1976), has been used to study coping strategies of older adults with a hearing impairment (Chen, 1994; Tolson \& McIntosh, 1997; Zhan, 2000). Unlike the theories of individual adaptation, these models emphasize 
that congruence occurs when the abilities of the individual match the demands and resources of the environment and that changes in either one may affect P-E Fit. As mentioned previously, the context of communication may affect the impact of a communication impairment. Older adults with a communication impairment, relieved of the stress of the work environment, may experience fewer difficulties as is reported in some research (Bricker-Katz, Lincoln, \& McCabe, 2010). Alternatively, an individual may experience the cumulative impact over time in health and communication ability and experience poorer fit with their environment and only be able to maintain their independence through increased assistance from others to avoid institutionalization (Schneider et al., 2010).

Comparing the five theories. As mentioned above, the theories described above are not mutually exclusive and demonstrate many themes in common. One of the key differences between them, however, is the focus of study. The unit of analysis for the first three theories is that of the individual. Although the context is acknowledged, the level of analysis is the individual's reaction to the environment. When applying the stress process model to analysis of caregiving relationships, for example, the caregiver is regarded as the unit of study and all other aspects of the environment (including the care recipient) are regarded as sources of stress or support which may affect coping (Pearlin, Mullan, Semple, \& Skaff, 1990). In comparison, Communication Accommodation Theory analyzes changes made at the level of both participants in a communicative exchange and is more reciprocal in its analysis. Similarly, the theory of P-E Fit considers changes made by both the Person and the Environment in considering the appropriateness of fit. Implicit to all of the theories, however, is the notion that accommodations made by the individual 
may be influenced by and, in turn, influence the environment. Consequently, an element of reciprocal influence is inherent to all models. As a result, it is difficult to disentangle which is cause and which is effect. For example, do more active coping styles prevent morbidity and disability and result in better health, or do healthier individuals have more time and energy deal with problems in a more active manner? Differentiating cause and effect is further complicated by the fact that coping strategies and levels of self-efficacy vary in the same individual across contexts and situations (Bandura, 1977; Eizenmann et al., 1997; Folkman et al., 1986). Perhaps more importantly, however, all theories acknowledge that individuals are not condemned to suffer the limitations and disabilities that are the inevitable result of disease (as conceptualized by the medical model of disability) but have varying degrees of agency to deal with the consequences of a health or communication problem in more positive or negative ways.

Summary. Communication disability includes a variety of disorders related to speech, language, hearing and cognitive function for communication. Communication impairments may be classified according to their etiology, which aspects of communication they affect, and also the age of onset at which they occur. Studies of communication in normal aging have shown that the conversational skills of normally aging older adults tend to remain well-preserved. With increasing age, however, the prevalence of conditions that may interfere with communication increases. It is estimated that $55 \%$ of Medicare beneficiaries (more than 16 million) have some type of communication disability. Communication impairments are associated with increased risk of depression, social isolation, loneliness, and poorer quality of life. They may affect a wide variety of domains of social function, including interpersonal relations, work, 
recreation, the ability to live independently, and the ability to make critical decisions such as those relating to healthcare. These findings have been confirmed in a variety of clinical populations. In their review of the relationship between communication impairments and aging, Kathryn Yorkston and colleagues (2010) distinguish between those experiencing "disability with aging" and those "aging with disability," depending on the point in the life-course at which the communication impairment occurs. The World Health Organization's ICF framework (2001) uses a biopsychosocial model to understand the interaction between the individual and the environment in the transition towards a disability which takes into account not only the contribution of biological factors but also psychological and social factors. In addition, the literature on disability offers number of theoretical models with regard to understanding the impact of an impairment and how people adapt to it. These models emphasize the need to understand the impact of personal and environmental factors on disability and, in turn, to consider the impact of a disability at a broader social level with regard to activity and participation.

\section{The Importance of Social Relationships for Mental and Physical Health}

The association between social relationships and health is now well-established. A recent meta-analytic review compiled the data on social relationships \& mortality from over 148 studies with more than 300,000 participants and found overall a $50 \%$ increased survival for individuals with stronger social relationships (Holt-Lunstad et al., 2010). These effects remained significant even after controlling for age, sex, initial health status, cause of death, and follow-up period. The authors noted that the magnitude of this effect is comparable to that associated with quitting smoking and that it is greater than many other known risk factors for mortality, such as physical inactivity and obesity. Ever since 
Berkman and Syme (1979) published their pioneering study linking social relationships to mortality using data on Alameda County residents, numerous studies have been conducted to further define the nature of the relationship and the pathways by which it acts (Berkman, Glass, Brisssette, \& Seeman, 2000; S. Cohen, 1988; Cohen, 2004; George, 1989; House et al.; Uchino, 2006; Uchino, Cacioppo, \& Kiecolt-Glaser, 1996). To date, however, the mechanisms underlying the effect of social relationships on health are not fully known.

Current conceptions of the relationship between social support and health tend to focus on two primary pathways: one related to the impact of social support on health behaviors, and the second related to social support and psychological health (Cacioppo \& Hawkley, 2003; Uchino, 2006). According to the first hypothesis, social support is health-promoting, because it facilitates and encourages healthier behaviors such as exercise, healthy diet, not smoking, and compliance with medical regimens. According to the second hypothesis, social support is health-promoting, because it results in more positive psychological health (e.g., with regard to appraisals or emotions and moods, such as depression, stress and feelings of control).

Given the interest in the potential importance of social relationships for health, a large number of measurement tools has been developed which focus on a variety of different types of social outcomes and can be divided into several different categories (Berkman \& Glass, 2000). Some studies have analyzed "functional" aspects of social relationships, referring to some of the many functions that are either provided or perceived to be available through social relationships. Others have focused on "structural" aspects of social relationships referring to the presence or absence of 
different types of social ties and roles indicating how connected the individual is to the society around him or her. The different types of relationships are summarized in Table 1.

Table 1

A Summary of Measures Used to Assess Social Relationships in the Health Literature

\begin{tabular}{|c|c|c|}
\hline $\begin{array}{l}\text { Type of } \\
\text { Measure }\end{array}$ & Term or Concept & Description \\
\hline \multirow[t]{4}{*}{ Functional } & & $\begin{array}{l}\text { Functions provided or perceived to be available by } \\
\text { social relationships }\end{array}$ \\
\hline & Received Social Support & $\begin{array}{l}\text { Receipt of emotional, information, tangible, or } \\
\text { belonging support. }\end{array}$ \\
\hline & Perceived Social Support & $\begin{array}{l}\text { Perception of availability of emotional, information, } \\
\text { tangible, or belonging support, if needed. }\end{array}$ \\
\hline & Loneliness & $\begin{array}{l}\text { Subjective feelings of isolation, disconnectedness, and } \\
\text { not belonging }\end{array}$ \\
\hline \multirow[t]{6}{*}{ Structural } & & $\begin{array}{l}\text { The existence and interconnections among different } \\
\text { social ties and roles }\end{array}$ \\
\hline & Marital Status & Presence absence of a spouse (or life partner) \\
\hline & Social Network Characteristics & $\begin{array}{l}\text { Network density, type, or size, or the number of } \\
\text { (supportive) social contacts. }\end{array}$ \\
\hline & Social Integration / Participation & $\begin{array}{l}\text { Participation in a broad range of social relationships, } \\
\text { activities, organizations, and/or social roles. }\end{array}$ \\
\hline & Living Alone & Living alone vs. living with others. \\
\hline & Isolation & $\begin{array}{l}\text { Pervasive lack of social contact or communication, lack } \\
\text { of participation in social activities, and/or lack of } \\
\text { confidant. }\end{array}$ \\
\hline \multicolumn{3}{|l|}{ Combined } \\
\hline & Multifaceted Measurement & $\begin{array}{l}\text { Multiple measures that combine two or more of the } \\
\text { measures above. }\end{array}$ \\
\hline & Social Capital & $\begin{array}{l}\text { An individual or collective characteristic that may refer } \\
\text { to individual or collective structure or function. The } \\
\text { term may refer to access to resources, information, and } \\
\text { support provided through social relationships and group } \\
\text { or organizational membership. Alternatively it may } \\
\text { refer to a collective attribute such as cooperation, social } \\
\text { cohesion, or trust. }\end{array}$ \\
\hline
\end{tabular}

Note: Adapted from "Social relationships and mortality risk: A meta-analytic review," by J. HoltLunstad, T.B. Smith, and J.B. Layton, 2010, PLos Med 7(7): e1000316, p. 9.

In general, the strongest relationships between social support and mortality have been reported when more complex measures of social integration have been used rather 
than a single predictor, such as binary assessments of marital status or living alone (HoltLunstad et al., 2010). These findings suggest that more detailed measures of social relationships may provide additional information about which specific aspects of social relationships influence health and mortality. Each of these types of social relationships and their importance is described in greater detail in the subsequent sections.

\section{Defining and Measuring Social Relationships}

Loneliness and social isolation. Most authors distinguish between loneliness and isolation. Loneliness is generally considered to be a subjective perception of inadequacy in the nature of one's social relationships, regardless of the number or nature of one's social contacts. In contrast, social isolation is a more objective measure which relates to shortcomings in the size or quality of an individual's social network (de Jong Giervald \& Havens, 2004). In general, loneliness and social isolation demonstrate relatively weak associations with each other. Socially isolated individuals do not necessarily perceive themselves as lonely, and lonely people are not necessarily socially isolated (measured objectively). In addition, Weiss (1974) differentiated between social and emotional loneliness. Social loneliness refers to the absence of companionship due to the lack of a feeling of connection to a broad network of friends and acquaintances. Emotional loneliness refers to the lack of feeling of intimacy with someone who is understanding and appreciative, such as with a spouse, confidant, or best friend. A number of researchers have used this distinction to investigate risk factors for different types of loneliness as well as investigate their relative importance (Dugan \& Kivett, 1994; Russell, Cutrona, Rose, \& Yurko, 1984; van Baarsen, Snijders, Smit, \& van Duijn, 2001). 
Stereotypical views had long depicted old age as a time of social isolation and loss, but more recent research indicates that this is not typical. Generally, studies indicate that there are relatively few changes in social relationships over time. Although the total number of social relationships decreases with age, the number of close social relationships does not and the amount of social support remains stable until very old age. Evidence suggests that only a minority of community-dwelling older adults is "severely" lonely or isolated (Grenade \& Boldy, 2008). Prevalence of "severe" loneliness in older adults (i.e. loneliness always or most of the time) is around $10 \%$ in most studies, although it may be higher in those living in low-income urban neighborhoods (15\%). Unlike loneliness, no universally-accepted criteria for measuring the severity of social isolation exist. Nonetheless, across multiple studies the prevalence of "severe" social isolation has been estimated to be around 10\% in community-dwelling older adults (Edelbrock et al., 2001; Hawthorne, 2008; Victor et al., 2000). Risk factors for loneliness and isolation include widowhood or loss of a partner, having no (surviving) children, living alone, deteriorating health, and significant negative life events. The risk of each of these life events increases over time and, consequently, the risk of loneliness and social isolation increases with advancing age (Dugan \& Kivett, 1994).

Neuroimaging studies of the brain demonstrate that feelings of social disconnection and loneliness cause the same types of emotional reactions as physical pain, suggesting the profound importance of a sense of connectedness for well-being (Eisenberger, Lieberman, \& Williams, 2003). Social isolation is now well-established as an independent risk factor for morbidity and mortality, both in the general population and in those with pre-existing disease (Cacioppo \& Hawkley, 2003; House, 2001). Like social 
isolation, loneliness is associated with health and well-being and some authors have argued that its impact has been underestimated (Cohen, 2000). Loneliness is often associated with depression but the two conditions are different and correlate with each other only moderately, leading some authors to conclude that loneliness is an independent risk factor for depression (Adams, Sanders, \& Auth, 2004; Mullins \& Dugan, 1990). Loneliness has also been shown to independently predict mortality as well as the likelihood of nursing home admission (Herlitz et al., 1988; Russell, Cutrona, de la Mora, \& Wallace, 1997).

\section{Social integration, social networks, and social network composition. Emil}

Durkheim's work on suicide (1897) provided the first evidence that social integration, or the degree to which an individual is connected to the groups that form a larger society, is important for health and well-being. Since then a considerable body of literature has developed to demonstrate that continued social connection is central to health, using various measures of social connectedness, such as volunteering, religious participation, group membership, and the presence of family ties (Adams \& Blieszner, 1995; Bassuk, Glass, \& Berkman, 1999; Berkman \& Syme, 1979; Krause, 2006a; Li \& Ferraro, 2005; Rowe \& Kahn, 1998). Many authors have used the terms social networks, social ties, and social integration almost interchangeably and have often made little distinction between them (Berkman et al., 2000). The early work in this area often used the term "social networks," although the standard methods and tools of network analysis were not utilized. Consequently a "second wave" of research developed in reaction to this early work in which a number of social scientists developed more detailed, functional analyses 
about why social relationships might contribute positively (or negatively) to health (Berkman et al., 2000).

Berkman and Glass (2000) define a social network as "the web of social relationships that surround an individual and the characteristics of those ties" (p. 145). The set of relationships in a social network typically includes friends, family members, neighbors, work associates, or other important individuals in that person's life (Bulmer, 1987). Social network analysis concentrates on the pattern of the relationship between individuals rather than the characteristics of the individuals themselves (Christakis \& Fowler, 2009; Hall \& Wellman, 1985). Social networks can be analyzed with regard to many different properties and characteristics including their size, density, and the nature of the interconnections. In terms of their importance, Lisa Berkman and colleagues (2000) provide a useful framework for understanding the relative importance of social networks, as shown in Figure 3.

According to this framework, social networks are embedded within the sociopolitical and cultural context. Social networks provide the interpersonal connections through which flow a number of different psychosocial mechanisms known to be important for health, including the provision of social support, social influence, social engagement, and attachment as well as access to resources and material goods. These, in turn, affect the more proximal pathways to health affecting health behaviors, psychological status, and physiologic responses (e.g., stress). Social networks are shaped by multiple factors over the life-course and "evolve organically from the natural tendency of each person to seek out many or few friends, to have large or small families, to work in 
personable or anonymous workplaces" (Christakis \& Fowler, 2009, p.13). Consequently, social networks are influenced by multiple factors, including age, gender, marital status, education, income, occupation, and personality (Allan, 1989; Burns \& Farina, 1984; Dickens \& Perlman, 1981; Mugford \& Kendig, 1987).

The social networks of older adults change over time. Although the total number of social relationships decreases with age, the number of close social relationships does not, and social support remains stable until very old age (Carstensen et al., 1999). Across a number of national studies the typical social network of older adults ranges between seven and ten people (Antonucci \& Akiyama, 1987; Mugford \& Kendig, 1987; Phillipson et al., 1998; Smith \& Baltes, 1998). In one study, Mugford \& Kendig (1987) developed a social network typology and investigated the influence of disability on network size and multiplexity (i.e. the number of different types of social network contacts). The authors reported that the presence of a disability of any kind influenced social network structure by increasing the number of kin (especially more distant relatives) in the social network, while decreasing the number of contacts with friends. 


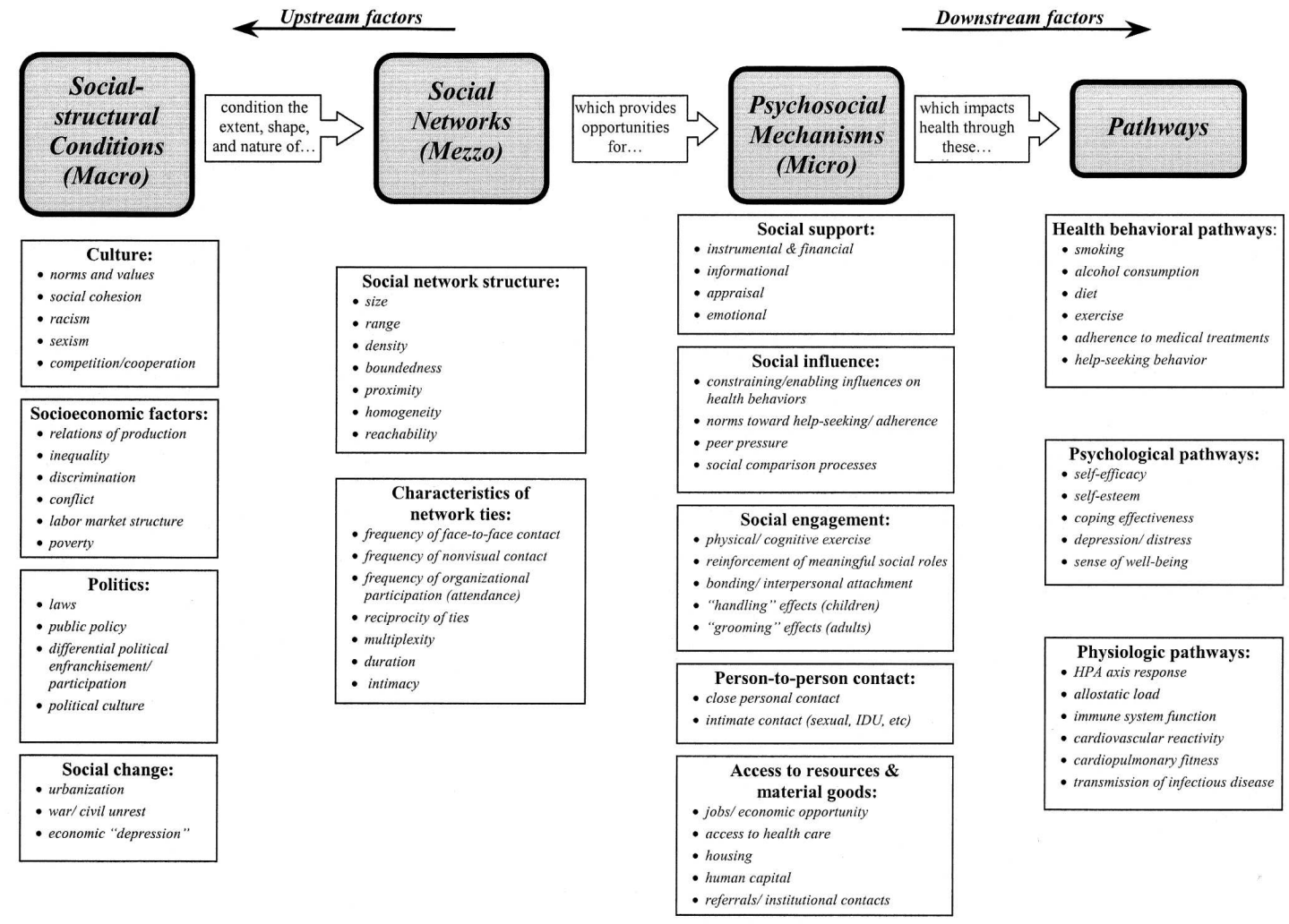

Figure 3. A conceptual framework for the impact of social networks on health. From "From Social Integration to Health: Durkheim in the New Millennium" by L.F. Berkman, T. Glass, I. Brissette, and T.E. Seeman, 2000, Social Science \& Medicine, 51, p.847.

According to research by Fiori and colleagues (2006) the depressive symptomatology of older adults varies by network composition. In their study, after controlling for other predictors, depressive symptomatology was significantly associated with social network variables. Depressive symptoms were highest in a group that had infrequent friendship contacts and lowest in those who had a diverse network composed of friends and family. Cornwell (2011) discussed the fact that while dense networks may provide a sense of "embeddedness" and access to social support, it may also provide obstacles to autonomy and privacy. Consequently he hypothesized that it may be important for older adults to maintain "bridging potential" in their social networks, i.e. 
ties to other adults who are otherwise poorly connected to each other. This concept that is similar to Granovetter's theory of the "strength of weak ties" (1973) which demonstrated that more casual acquaintances outside an individual's social circle might provide access to information, companionship, and other resources that are not otherwise available.

Previous research has shown that kin and nonkin frequently serve very different functions in the lives of older people (Crohan \& Antonucci, 1989). In particular, nonkin contact seems to be particularly important for feelings of well-being and self-esteem (Johnson \& Catalano, 1983; Lee, 1979; Wood \& Robertson, 1978) whereas kin are more important for providing practical and material assistance that may be critical for delaying or preventing institutionalization (Tobin \& Kulys, 1981). These differences in the functions of kin and nonkin in the lives of older adults probably means that the types of support provided by each group differ substantially, and this is reinforced by the fact that older adults participate in very different types of activities with kin and nonkin (Larson, Mannell, \& Zuzanek, 1986).

This observation has led to some debate about the importance of different kinds of relationships. In particular, there have been two counterpoised perspectives towards the issue of particular types of bonds. Litwak and his colleagues have previously argued that particular relationships lend them to certain kinds of functions and thus, the absence of a particular relationship may result in a "lost function" (Dono et al., 1979; Litwak, 1985). In contrast, Cantor $(1979,1980)$ has posited an alternative "hierarchical compensation model" in which more distant bonds (e.g., nonkin) become relatively more important when closer bonds (e.g., kin) are unavailable. In this model, functions are more likely to 
be transferred than lost and there is an explicit ordering of responsibility from spouses and children to other relatives, friends and neighbors (Cantor, 1979; Chatters, Taylor, \& Jackson, 1985; Kendig, Coles, Pittlekow, \& Wilson, 1988; Shanas, 1979; Stoller \& Earl, 1983; Townsend, 1963). Studies that have examined the association between the impact of support and who it is provided by have shown that, although most social provisions are valuable regardless of source certain types of support were more beneficial when provided by certain types of providers (Felton \& Berry, 1992). In addition, networks in which fewer providers provide more functions ("multiplexity") are associated with poorer well-being (Felton \& Berry, 1992). Even though, according to the hierarchical compensation model, older adults may be able to negotiate for their support needs these studies suggest that not all support is equally beneficial and that there may be negative consequences for mental health.

Social support. An extensive body of literature demonstrates the consistent relationship between social support and better physical health (Berkman et al., 2000; S. Cohen, 1988; House et al., 1988; Seeman, 1996; Uchino, 2004). Individuals with low levels of social support have higher mortality rates, particularly from cardiovascular disease but also due to other causes such as cancer and infectious diseases (Uchino, 2006). Social support is related to mental and physical health and also to the risk of hospitalization and institutionalization (Tobin \& Kulys, 1981). Social support is also related to recovery and rehabilitation, such as disability after a stroke (Kwakkel, Wagenaar, Kollen, \& Lankhorst, 1996).

Currently, with many different definitions and categorizations of social support, no one model is universally accepted (Uchino, 2006). Some models focus on the different 
functions that social relationships may perform. One of the most commonly cited is Robert Weiss's (1974) model of "social provisions" which encompasses other widely used conceptualizations of social support (Cobb, 1979; Cohen \& Wills, 1985; Cutrona \& Russell, 1987; Kahn, 1979, Schaefer, Coyne, \& Lazarus, 1981). According to Weiss, six different social functions or "provisions" may be derived from interactions with others. All six of these provisions are necessary for an individual to feel adequately supported and avoid loneliness. At the same time, different provisions may be more or less important at different points in the life-course and more than one individual may provide more than one type of provision. Each of these six provisions, namely attachment, social integration, reassurance of worth, reliable alliance, guidance, and opportunity for nurturance, is discussed in greater detail below.

The first, attachment, is defined as the sense of emotional closeness derived from a sense of security, most commonly from a spouse. This function may also be derived from close friends or other familial relationships. Social integration relates to a sense of "belonging" which derives from membership in a group of individuals with similar interests, concerns, and/or recreational activity. This function is most often provided by friends. Reassurance of worth is the sense that an individual's competence, skill, and value are recognized by others. Reliable alliance is the sense that others can be counted on to provide assistance in time of need, and is most often provided by family members. Guidance is the provision of advice or information that may come from numerous sources, often in earlier life from teachers, mentors or parent figures. Finally, opportunity for nurturance is the sense that others depend on the individual for their well-being. It is often associated with parenting but can also be associated with the provision of care to a 
spouse or other family members. Even though models of social support use a variety of terminology, many similarities exist across the various models used in the research todate (Cohen, Underwood \& Gottlieb, 2000; Cutrona \& Russell, 1987). Weiss' typology is useful because it covers the main types of social support provision found in other models. Social support is typically divided into various subtypes, such as emotional, informational, tangible/instrumental, belonging, and appraisal support which are comparable to Weiss' six provisions, as defined above and listed in Table 2 below.

Table 2

Comparing Component Models of Social Support Across Authors

\begin{tabular}{|c|c|c|c|c|}
\hline $\begin{array}{l}\text { Weiss } \\
(1974) \\
\end{array}$ & $\begin{array}{l}\text { Cobb } \\
(1979)\end{array}$ & $\begin{array}{l}\text { Kahn } \\
(1979)\end{array}$ & $\begin{array}{c}\text { Schaefer et al. } \\
\text { (1981) }\end{array}$ & $\begin{array}{c}\text { Cohen \& Wills } \\
(1985)\end{array}$ \\
\hline Attachment & Emotional support & Affect & Emotional support & \\
\hline Social integration & Network support & & & Belonging suppor \\
\hline Reassurance of worth & Esteem support & Affirmation & & $\begin{array}{l}\text { Self-esteem } \\
\text { support }\end{array}$ \\
\hline Reliable alliance & Material support & Aid & Tangible aid & Tangible support \\
\hline Guidance & $\begin{array}{l}\text { Instrumental } \\
\text { support }\end{array}$ & & $\begin{array}{l}\text { Informational } \\
\text { support }\end{array}$ & Appraisal support \\
\hline $\begin{array}{l}\text { Opportunity for } \\
\text { nurturance }\end{array}$ & Active support & & & \\
\hline
\end{tabular}

Adapted from "The Provisions of Social Relationships and Adaptation to Stress," by C.E. Cutrona and D.W. Russell, 1987, Advances in Personal Relationships, 1, p.43.

Another distinction in the literature is between received and perceived support.

Received support refers to the assistance and aid that has actually been provided to an individual. Perceived support refers to an individual's perception of whether help would be available if it were needed. Research demonstrates that these two concepts are only moderately correlated (Dunkel-Schetter \& Bennett, 1990) and some studies have reported that perceived support may actually be more important than received support in predicting health outcomes (Barrera, 1986, 2000; Kaul \& Lakey, 2003; Lakey \& Lutz, 1996; Reinhardt, Boerner, \& Horowitz, 2006; Sarason \& Sarason, 1986; Uchino, 2004). 
These variations in the definitions and measurement of support reflect a lack of consensus about which aspects of social support are most important and how they function to impact health outcomes.

Positive and negative social exchanges. Social network members are an important source of practical support and emotional companionship in times of need (Krause, 2006b).The majority of the social support literature has assumed that all social relationships are positive and, therefore, have not assessed the quality of those relationships (Holt-Lunstad et al., 2010). At times, however, the support provided by network members can be perceived to be intrusive or insulting and may become a source of conflict. Evidence from daily diary studies show that conflict with other members of the social network may be one of the most upsetting sources of stress experienced in daily life (Bolger, DeLongis, Kessler, \& Schilling, 1989). As a result there has been increasing recognition of the fact that some relationships may be harmful for health (Rook, 1998).

This has led to a focus on the nature of the interactions between social network members and their implications for health. The term positive social exchanges has been used to describe the various aspects of positive support, such as emotional support, informational support, instrumental support, as described in the previous section. This has been contrasted with a wide variety of negative actions such as personal criticism, intrusiveness, and rudeness, as well as physical and financial abuse. These actions have been variously labeled as negative social exchanges, social strain, interpersonal conflict, or negative or harmful social support. Negative exchanges are associated with psychological distress and are strongly associated with symptoms of chronic strain and 
acute stress (Finch, Okun, Pool, \& Ruehlman, 1999; Krause \& Rook, 2003; Rook, 1998). Marriages that have high levels of conflict and disagreements are associated with higher levels of depression and poorer immune function in spouses (Kiecolt-Glaser, 1999; Marsland, Bachen, Cohen, \& Manuck, 2001). Other problematic personal relationships are also associated with a wide range of health problems (Cohen, 2004).

Both positive and negative social exchanges have an impact on psychological health in older adults (Newsom, Rook, Nishishiba, Sorkin, \& Mahan, 2005). The detrimental effects of negative social exchanges often outweigh the beneficial effects of positive social exchanges (Rook, 1998). Negative interactions occur in the lives of older adults both with and without health impairments (Newsom, 1999; Rook, 1984). For older adults with health limitations, care recipients do not always perceive interactions involving assistance as being helpful or beneficial (Newsom \& Schulz, 1998). This, in turn, may cause them to be less satisfied with their social support system (Krause, 1995). Overprotective behavior or the provision of more instrumental support than is necessary may result in "learned helplessness," decreased self-esteem, and perceptions of incompetence which, in turn, may foster higher levels of self-perceived disability, increased distress, and lower levels of subjective well-being (Baltes, 1996; Martire, Stephens, Druley, \& Wojno, 2002; Newsom, Mahan, Rook, \& Krause, 2008; Reynolds \& Perrin, 2004; Seeman, Bruce, \& McAvay, 1996). These findings, relating to negative social exchanges or "miscarried support," may explain one of the consistent findings from the social support literature, namely that the perceived availability of support is a better predictor of positive outcomes than the actual amount of received support. 
Social capital. Social capital is a term that has been used extensively in fields such as sociology, economics, and political science, but only more recently has the term been widely applied to public health (Kawachi, Subramanian, \& Kim, 2008). The term social capital has been used to refer to the bonds between individuals that make it possible for individuals and groups to achieve a variety of goals (Kim, 2008). Such bonds are thought by some to have health promoting effects. However, a consensus about the definition of social capital as a concept is lacking. Coleman (1990) defined social capital not as a single entity but as a "variety of different entities" (p. 302) all of which share two common characteristics. First, they all relate to some aspect of social structure and second, the fact that the actions of separate individuals are facilitated by being part of that social structure. Social capital can be conceptualized as an individual asset which connects it with other research on social networks, social support, and social influence and their connections to health (Eriksson, 2011). Social capital can also be defined as a collective attribute with regard to groups and local communities. The latter approach emphasizes the connection between income inequality, social cohesion, social infrastructure (e.g., housing), access to healthcare services, and health information and individual health. In a systematic review of studies investigating the relationship between social capital and physical health, the strongest associations were found to be between individual social capital and health (Kim, Subramanian, \& Kawachi, 2008). In contrast, studies of collective social capital and health have shown inconclusive results (Islam, Merlo, Kawachi, Lindström, \& Gerdtham, 2006). Some of these inconsistencies may relate to differences in conceptualization, operationalization, or the need for multi-level analysis in this type of research (Engström, Mattsson, Järleborg, \& Hallqvist, 2008; 
Poortinga, 2006). Nonetheless, the literature on social capital adds an important perspective to the health literature by emphasizing the connection between health and broader social characteristics, such as the economic and political environment. In general, the social support literature has tended to ignore the extent to which social connections are a mechanism for access to material goods, financial support, and other resources (Berkman et al., 2000), but there is an extensive literature documenting an association between income inequality, health, and mortality (Kawachi, Kennedy, Lochner, \& Prothrow-Stith, 1997). Consequently, social capital is a useful concept for understanding individual health within a sociopolitical and economic context. Income inequality at the individual and the neighborhood level has both been shown to predict mental and physical health in later life (Kahn \& Pearlin, 2006; Muramatsu, 2003).

Social participation. A considerable body of historical research demonstrates that social activities directly enhance well-being independent of levels of life-stress (Andrews, Tennant, Hewson, \& Vaillant, 1978; LaRocco \& Jones, 1978; Lin, Simeone, Ehsel, \& Kuo, 1979; Miller, Ingham, \& Davidson, 1976). This work has established the importance of social participation or social engagement for well-being. Social participation or social engagement refers to the ability of an individual to participate in social roles, relationships, activities and functions. These activities and functions typically involve two or more individuals during which social exchanges occur. Social participation is associated with decreased morbidity and mortality, and increased quality of life (Berkman, 1995; Berkman et al., 2000; Levasseur, Desrosiers, \& St-Cyr Tribble, 2008). Social participation is also highly valued by older adults and is considered a key 
outcome with regard to rehabilitation for many different kinds of health problems and disabilities (Dalemans et al., 2008; Levasseur, St-Cyr Tribble, \& Desrosiers, 2009).

According to the WHO's ICF model (2001), an individual's health and well-being can be described with regard to both body functions and structures, but also in terms of the activities and participation that the individual participates in. The term "activities" usually refers to specific behaviors that the individual may undertake, while "participation" refers to the social roles that are gained through performing those activities (Dalemans et al., 2008). As a result, the WHO has targeted the enhancement of social participation by older adults as part of its policy framework in addressing concerns about population aging (2002). Despite considerable research in this area, however, wide variations are found in the definition of social participation (Levasseur et al., 2010). As with social support, the variations in conceptualization attest to the great diversity of perspectives that have been applied to this area from a number of different academic fields, as well as a lack of understanding about which aspects of social participation are most important for the outcome of interest.

\section{Theories of Social Relationships and Aging}

Social relationships and aging. The social networks of older adults demonstrate great diversity (van Tilburg, 1998). The theoretical perspectives that have been used to account for the differences in the social networks of older adults can be subdivided into three main categories (van Tilburg \& Thomese, 2010). These three perspectives emphasize: a) social and personal transitions in later life; b) changes in expected returns within the network; and, c) proactive management of personal relationships at the individual level. 
In the first perspective, social networks can be understood with regard to social and personal transitions in later life. This view emphasizes the life-course perspective and the Convoy Model (Kahn \& Antonucci, 1980), which conceptualizes the individual as part of a "convoy" of individuals with whom he or she has developed relationships from childhood to very old age. Over the life course, some relationships end (e.g. due to widowhood, retirement, or divorce) and others are created (e.g. due to marriage, a new job). Consequently, the social network of older adults reflects the cumulative impact of numerous events as well as individual choices about maintaining or relinquishing particular relationships. The ability to maintain relationships, however, may be affected by personal and social factors such as health, geographic location, and role changes.

The second perspective is based on Exchange Theory (Blau, 1964; Homans, 1958; Thibaut \& Kelley, 1959), emphasizing that many relationships are governed by norms of equality and fairness. Drawing on the work of these theorists, problems of aging were seen as problems of decreasing power resources (Dowd, 1975). If older adults were to become dependent on others and could not reciprocate for their care, the resulting disruption in the balance of their relationships would cause strain and discomfort. The imbalance in this relationship could also result in the loss of autonomy as the care recipient might be forced to exchange compliance for their continued care. Imbalance may not necessarily cause a relationship to end, however, as disparity in support to an older adult may be normatively accepted and even viewed as desirable (van Tilburg \& Thomese, 2010). In addition, older adults might be able to maintain norms of reciprocity as they age by providing other forms of support to their families in return such as financial support, childcare, or sharing housing (Connidis, 2010; Silverstein, Conroy, 
Wang, Giarrusso, \& Bengtson, 2002) and so be able to maintain a sense of autonomy and status within the family. Reciprocity within parent-child relations has been frequently investigated with regard to the understanding transfers of time, labor, and financial assets across generations (Antonucci, 1990; Henretta, Hill, Li, Soldo, \& Wolf, 1997; Morgan, Schuster, \& Butler, 1991; Whitbeck, Simons, \& Conger, 1991). This research provides some evidence that elements of both repayment and altruism are at work in governing the normative behavior of adult children (Silverstein et al., 2002). Evidence from the caregiving literature also supports the notion that dependent older adults recognize norms of reciprocity and attempt to repay a caregiving spouse by providing emotional in return for instrumental support (Archbold, Stewart, Greenlick, \& Harvath, 1990; Wright \& Aquilino, 1998).

The third perspective on social relationships and aging emphasizes that changes in an individual's social network occur due to changes in the individual's motivation and preferences. Socioemotional Selectivity Theory was formulated to explain the finding that there is a consistent reduction in social interaction in older adults (Carstensen, 1991). Two previous theories had attempted to account for this phenomenon. Activity theory considered this to be the result of social norms and a marginalization of older adults by the sociopolitical system, as evidenced by mandatory retirement (Havighurst \& Albrecht, 1953). Disengagement theory, on the other hand, suggested that older individuals psychologically withdrew from society as a way of preparing for death (Cumming \& Henry, 1961). According to Socioemotional Selectivity Theory, however, this phenomenon is the result of a change in priorities due to advancing age which leads to a prioritization of close, rewarding personal relationships over those which are more 
negative or superficial (Carstensen et al., 1999). According to this view, social relationships serve a number of roles, including those that are more informational in nature and those that are more emotional. The perception of time plays a role in the prioritization between these two types of relationships. Younger adults frequently prefer more informational types of relationships/experiences due to their potential for novelty and learning, while older adults typically prefer more familiar and emotional relationships/experiences (Frederickson \& Carstensen, 1990). This preference appears to be related to the perception of time as, when the same choice was explored under the hypothetical situation of extended life expectancy, the preferences of older adults became identical to those of younger adults (Fung, Carstensen, \& Lutz, 1998). Unlike previous theories, therefore, Socioemotional Theory considers the role of cognitive and motivational factors in understanding the changes in social relations across the lifecourse. Changes in network size and composition are seen to be the result of "proactive aging" in which social relationships are managed (Lang \& Carstensen, 1994). Socioemotional Selectivity Theory is consistent with the model of Selective Optimization with Compensation and also the life-course perspective (Baltes, 1987, 1997). It is further supported by evidence that suggests that individuals become more positive about their social relationships as they age despite reductions in social network size (Antonucci, 2001).

To summarize the key findings of all three approaches, therefore, it is clear that the social networks and social relationships of older adults reflect a number of factors across the life-course. These factors include individual choices and life-events that have shaped the pattern of connections with kin and non-kin in early and mild-adulthood. 
Other important factors include interpersonal negotiations to maintain balance and equity in those relationships and avoid feelings of dependency. In addition, cognitive and motivational factors may affect judgments by older adults about resource allocation and satisfaction in determining which relationships to sustain.

\section{The Impact of Communication Impairment on Social Relationships}

A considerable body of evidence indicates that communication impairments are associated with poor mental health and well-being across a variety of populations. One reason for this may be the impact of communication impairments on social relationships. In this section, the literature relating to the association between communication impairments and social relationships is reviewed, including findings from descriptive studies, as well as studies of loneliness, social isolation, social network size and composition, social support, positive and negative social exchanges, and social participation.

Communication impairments and personal relationships. The impact of worsening communication may have further negative impacts on intimate relationships. In a cross-sectional study of spouses of individuals with Parkinson's Disease, frustration due to communication difficulties increased significantly by stage of the disease and this paralleled other changes, including declines in lifestyle and mutuality and increases in caregiver strain (Carter et al., 1998). Declines in speech intelligibility over time have been shown to be associated with declines in marital quality in a longitudinal study of individuals with ALS and their partners (Joubert et al., 2011). In many studies of spouses of a partner with a hearing loss, a variety of negative outcomes have been reported, including increased interpersonal strain, irritation/effort, stress, anger, resentment, blame, 
limitations in family roles and activities, negative self-esteem of the spouse, and a restricted social life as a couple (Hétu et al., 1993). Consequently, the negative impact of a communication impairment appears to not just be limited to the affected individual but may also have significant effects on a partner also. Wallhagen and colleagues (2004) conducted a longitudinal assessment of older married couples from the Alameda County Study and found that, even after controlling for health and demographic characteristics, hearing impairment was associated with poorer physical, psychological, and social wellbeing in partners five years later, perhaps as a result of the cumulative toll of interpersonal strain.

Despite the considerable heterogeneity of different types of communication impairments, the literature relating to the impact on different types of social communication and contexts demonstrates many commonalities (Baylor et al., 2011). In a qualitative study of the impact of communication impairments on close relationships (Bute et al., 2007), friends and family members of individuals with a variety of different types of impairments were interviewed. They reported that the participants made a wide variety of accommodations and adjustments to continue their relationships. These included adjusting the mechanics of communication, managing topics, using a third party as an intermediary, and inferring meaning from a wide variety of cues. Across these various studies, it appears that the cumulative impact of these changes frequently includes a feeling of increased work to maintain a relationship, decreased feelings of closeness and intimacy, and changes in the ability to perform familial and social roles. These changes, in turn, are often associated with feelings of a change in personality or a perception of a loss of the former sense of self. 
Loneliness and social isolation. Among a number of risk factors for increased loneliness, numerous studies provide evidence that loneliness is experienced by many adults with communication difficulties (Balandin, Berg, \& Waller, 2006; Ballin \& Balandin, 2007; Parr, 2007; Yorkston et al., 2010). Feelings of loneliness can be differentiated from depression but, like depression, loneliness is strongly associated with the mental and physical health of older adults. Loneliness is also a predictor of other negative outcomes, such as psychological distress, as was found in a longitudinal study of older adults recovering from a stroke (Hilari et al., 2010). As with other social outcomes, to date the largest body of evidence relates to older adults with hearing loss. The presence and severity of a hearing impairment has been associated with subjective and objective measures of social isolation (Weinstein \& Ventry, 1982). Older adults with a hearing impairment often choose to avoid social situations and activities in which they have trouble communicating due to fears of ridicule and stigma, leading to reduced social participation, loneliness, social isolation and withdrawal (Hétu et al., 1993).

Some contradictions appear in the literature, however. Evidence for significant associations between hearing loss and loneliness have been found in some studies (Kramer, Kapteyn, Kuik, \& Deeg, 2002; Hawthorne, 2008; Strawbridge, Wallhagen, Shema, \& Kaplan, 2000; Wallhagen, Strawbridge, \& Kaplan, 1996), but not in others (Kivett, 1979; Berg, Mellström, Persson, \& Svanborg, 1981; Nachtegaal et al, 2009). Possible reasons for these discrepancies include differences in study design, instrumentation, study populations, and covariates in the analysis. Some studies have hypothesized that certain groups may be more at-risk than others, but again there are contradictions in these findings (Chen, 1994; Pronk et al., 2011). Intervention studies 
provide further evidence of a possible causal association between communication status and loneliness (Mulrow, Aguilar, Endicott, Tuley, et al., 1990; Poissant, Beaudoin, Huang, Brodsky, \& Lee, 2008).

Two potential reasons for the association between communication impairments, social isolation, and loneliness can be hypothesized. Reports from friends and family members of individuals with communication impairments report a change in the nature and content of communication, such as the fact that conversation becomes more practical and less intimate due to the "work" of communicating (Bute et al., 2007; Hétu et al., 1993). This would suggest that a decline in the quality of personal relationships leads to increased "emotional loneliness." In addition, a loss of companionship and friendship may occur due to a reduction of the presence of friends in the social network (as discussed below) leading to increased feelings of "social loneliness." This might also be associated with increased social isolation due to social withdrawal, as is often reported (Hétu et al., 1993; Yorkston et al., 2010). The lack of differentiation between the two different types of loneliness may be one of the reasons for the inconsistencies in previous research.

Social network size and network composition. To date, changes in social networks in individuals with a communication impairment have not been extensively investigated (Worrall \& Hickson, 2003). To date there have been a few studies that have examined the association between communication-related variables and social network size in community-dwelling older adults (Hickson et al., 1995; Lind et al., 2003). The findings from these studies have been relatively unremarkable, however, and have not shown associations between communication-related variables and social network size. 
There are a number of possible reasons for this, such as the fact that these studies had small sample sizes, the participants tended to be relatively healthy and had a low incidence of communication impairment, and the fact that the studies did not use global measures of everyday communication. Objective measures of hearing and cognitive function were not strongly associated with social network measures, although individuals with dual sensory impairment (i.e., both hearing and visual impairment) were found to have significantly smaller and more restricted social networks than those with normal vision and hearing (Lind et al., 2003).

In contrast, a wealth of qualitative and descriptive literature exists relating to the problems with personal relationships experienced by individuals with communication impairments of varying kinds. As mentioned previously, older adults with a hearing impairment often choose to avoid social situations and activities in which they have trouble communicating, leading to social isolation and withdrawal (Hétu et al., 1993; Weinstein \& Ventry, 1982). In addition, reduced social network size has been documented in stroke survivors (Davidson et al., 2008). Approximately $30 \%$ of stroke survivors have aphasia, which is a communication disability that results from the brain damage caused by the stroke and which may have a permanent impact on speech, language and literacy. Typically, older people with aphasia communicate with fewer friends and have smaller social networks. Approximately $30 \%$ of individuals with aphasia report that they have no friends a year after the onset of the disorder (Hilari \& Northcott, 2006). Friendships often end because friends no longer know how to communicate with the individual with aphasia (Parr, 2007). 
Previous research in older adults with other kinds of disability has demonstrated that the presence of a disability is associated with an increase in the number of kin in the social network, and a decrease in the number of friends (Mugford \& Kendig, 1987). This may place older adults at-risk for poorer psychological health. Research conducted both with older healthy adults and older adults with disabilities has suggested that network composition is strongly associated with mental health and that individuals who have social networks that are primarily composed of kin tend to have more depressive symptoms and worse affect (Felton \& Berry, 1992; Fiori, McIlvane, Brown, \& Antonucci, 2006; Mcllvane \& Reinhardt, 2001). This is an area that remains to be researched with regard to communication impairments.

In one of the few studies on this topic, Gordon Blood and his colleagues (1994) compared a number of variables including social network size, social support, self-rated communication, and psychological adjustment in survivors of laryngeal cancer. Individuals who were well-adjusted scored significantly higher on measures of social support, self-rated communication, and had significantly larger social networks than those that were more poorly adjusted. Because this was a cross-sectional study, however, it is not clear whether differences in social network size and social support were the cause or the effect of other variables. In summary, some limited evidence suggests that the presence of a communication impairment may be associated with social network size and composition, but the findings to date are far from conclusive. In addition, the implications of differences in social network size and composition for adults with communicatin impairments are largely unknown. 
Social support. In one of the largest studies conducted to date, the presence of a hearing impairment was a significant predictor for the use of community support services as well as support from friends, family and community members, even after controlling for health, disability, and demographic characteristics (Schneider et al., 2010). In explaining the association between hearing loss and the need for increased support of all kinds, the authors speculated that this could be due to hearing loss altering the nature and quality of communication, social interactions, and feelings of self-sufficiency.

Communication impairments may also cause a decline in the quantity and quality of available support over time. Pachana and colleagues (2008) conducted a large longitudinal study of older Australian women to identify factors that would affect social support for older women over a three-year period. Over time, the presence of a hearing impairment was not associated with support network size but was associated with a significant decrease in satisfaction with social support. It is not known what may have caused this decline in satisfaction, but the authors speculate that it could be due to an unwillingness or inability to participate in meaningful social interactions or a decreased ability to derive pleasure from these interactions. Nonetheless, these findings suggest that hearing impairment may be one of a number of factors that cause an erosion in the quality of available support over time.

In many studies, social support has emerged as a powerful, independent predictor of psychological health in older adults with hearing impairments, but the exact nature of that relationship is unclear. In a study of the relationship between sensory loss, family support, and mental health in the elderly, Oppegard and colleagues (1984) conducted a study of community dwelling older adults. Respondents were subdivided into two groups 
based on their levels of social support. Both hearing impairment and visual impairment were significantly correlated with anxiety and depression, but only for those with low levels of social support. The authors concluded that this is consistent with the "stressbuffer" hypothesis of social support. In another study, however, these findings were not replicated. Frankel and Turner (1983) conducted a cross-sectional study of communitydwelling adults diagnosed with adult-onset hearing impairment to determine what factors were associated with psychological distress. Social support emerged as the most important predictor. When stratified by severity of hearing loss, the relationship between social support and psychological distress remained consistent regardless of the severity of the hearing impairment. This finding was inconsistent with the findings of Oppegard and colleagues (1984) who found that the association between social support and psychological distress did differ based on hearing impairment severity. As the authors point out, however, the cross-sectional nature of the study and the fact that all three measures were self-reported suggests that it is possible that all three reflect the impact of distress. Consequently, the role of social support and the direction of the relationship between the three variables is unclear.

Positive and negative social exchanges. Previously studies have shown that the presence of a physical disability is associated with an increase in negative social exchanges over time (Mavandadi, Rook, \& Newsom, 2007). A large body of literature indicates that individuals with communication impairments and their conversational partners experience a wide variety of difficulties interacting with each other on a daily basis. Based on the literature, however, it is possible to hypothesize three different 
scenarios with regard to the impact of a communication impairment and the relative frequency of positive and negative social exchanges.

It is possible that individuals with communication impairments experience fewer positive social exchanges. In a large number of progressive neurological conditions, declines in communication have been shown to have negative impacts on intimate relationships including declines in marital intimacy and satisfaction, increased frustration, and increased caregiver strain (Baikie, 2002; Carter et al., 1998; Joubert et al., 2011). In studies of spouses of a partner with a hearing loss, poorer relationship quality has been found to be associated with hearing loss, including increased interpersonal strain, irritation/effort, stress, anger, resentment, blame, limitations in family roles and activities, negative self-esteem of the spouse, and a restricted social life as a couple (Hétu et al., 1993). These studies suggest that communication impairments are associated with more negative social exchanges and fewer positive social exchanges on an ongoing basis.

Alternatively, it is possible that communication impairments may be associated with a decrease in all social exchanges (i.e., both positive and negative) as a result of social withdrawal, which is a frequent consequence of many conditions (Yorkston et al., 2010). The extent to which withdrawal may be used as a protective mechanism by individuals with communication impairments is not well-known. In preliminary data for the current study, the presence of a communication impairment was found to be a significant predictor of fewer positive social exchanges, even after controlling for age, gender, partnership status, health, and functional limitations. No significant difference was found for negative social exchanges, however. 
It is also possible to speculate that individuals with communication impairments might experience an increase in positive social exchanges. First, individuals with communication impairments may experience an increase in social support as a result of their health condition (Fletcher et al., 2012), a phenomenon known as "support mobilization" (Eckenrode, 1983). Second, interviews with friends and family members of individuals with a communication impairment demonstrate that conversational partners may make significant accommodations to sustain their personal relationship and maximize the quality of their interactions (Bute et al., 2007). Third, the perspective of individuals with a communication impairment may change over time with the result that they may value everyday interactions more highly or become more invested in activities that do not involve communication (Cruice, 2002; Parr, 2007; Stephens \& Kerr, 2003). Although not widely studied, any of these processes could be responsible for an increase in positive social exchanges and a decrease in negative exchanges associated with communication impairment. In short, to date little is definitively known about the association between social network size or other network characteristics and the frequency of different types of social exchanges in adults with communication impairments.

Social participation. The WHO has targeted the enhancement of social participation by older adults as part of its policy framework in addressing concerns about population aging (WHO, 2002). Definitions of social participation vary widely (Levasseur et al., 2010) and there is no consensus about which measures may be the most appropriate outcome tool for adults with various kinds of disabilities (Dalemans et al., 2008; Dijkers et al., 2000). With regard to communication impairments, the findings from 
previous research have been mixed. Cruice and colleagues (2005) conducted a crosssectional study of the social participation of community-dwelling older adults. Most of the sample had mild word-retrieval and hearing deficits but neither of these variables was predictive of either communicative participation or social participation in the regression model. The strongest predictors of communicative participation were age, vision, and education. These same variables, in conjunction with depressive symptoms, were the strongest predictors of social network size and social participation. The authors were surprised that neither of the communication-related measures (i.e., word retrieval and hearing) was associated with participation. Possible reasons for the lack of an association include the fact that the sample as a whole was relatively healthy and the deficits on both of the communication-related measures were generally mild.

The lack of an association between communication-related variables and participation reported by Cruice and colleagues (2005) is in contrast to the findings from clinical populations. An association between communication impairment and participation was found in a randomized controlled trial of older adults with hearing impairment who were followed after receiving a hearing aid (Mulrow, Aguilar, Endicott, Tuley, et al., 1990). Those in the hearing aid group demonstrated significant improvements in social, emotional, and communicative function. Significant improvements in the hearing aid group were also found for measures of cognition and depression. No improvements occurred for those on the waiting list. These findings provide strong support for a causal association between hearing loss and alterations in social and communicative participation in hearing impaired older adults. 
Baylor and colleagues (2010) conducted a large, cross-sectional survey of older adults with multiple sclerosis. Respondents were asked to rate how much multiple health, demographic, and disease-related characteristics interfered with participation in a variety of social situations in which communication was required. Of the thirteen variables assessed, six were found to be significantly associated with participation, namely: fatigue, slurred speech, depression, problems thinking, employment status, and social support. These six variables accounted for nearly $49 \%$ of the variance on the participation measure. In this case, a communication-related variable ("slurred speech") was a significant predictor of participation even after controlling for the other characteristics. Reasons for the inconsistencies in findings between the studies may include differences in the study populations, study measures, and the other variables included in the regression model. Compared to studies of community-dwelling older adults, therefore, studies of individuals with communication impairments suggest there is an association between communication status and social participation.

Comparing hearing impairment to other chronic conditions. The relative impact of communication disorders on psychosocial health, disability, and quality of life is difficult to estimate because they often co-occur with other health problems and functional limitations. Interviews with individuals with disabling conditions that cause physical as well as communication impairments, for example, emphasize that communication is just one "part of the picture" (Walshe \& Miller, 2011). Some research has analyzed the relative impact of a hearing impairment compared to other chronic health conditions on a range of psychosocial outcomes. Kramer and colleagues (2002) examined the impact of chronic diseases on several psychosocial measures in a large 
sample of older adults. The outcomes of interest were depression, self-efficacy, mastery, loneliness, and social network size. After controlling for sociodemographic covariates and comorbidities, hearing impairment was a significant predictor of all five outcomes. Specifically, hearing impaired people reported more depressive symptoms, lower feelings of self-efficacy and mastery, more loneliness, and a smaller social network than their normally-hearing peers. Other chronic conditions also showed significant associations with some of the outcomes, but only hearing impairment was significantly associated with all five psychosocial measures. Similarly, Ormel and colleagues (1997) examined predictors of psychological distress using data from a large sample of communitydwelling, older adults. They examined the impact of a total of 16 chronic health conditions, including impairments in vision, hearing, and cognition. Of all of the conditions studied, hearing impairment was most strongly associated with distress. In addition, the presence of a hearing impairment was associated with higher levels of physical and role disability, and with lower levels of mastery, self-efficacy, and social support. Multiple regression results were consistent with a model in which the mental health effects of a hearing impairment were mediated by reduced mastery and selfefficacy. In explaining this relationship, the authors suggest that hearing impairments not only limit participation in various kinds of activities but also lead to declines in the sense of control, competence and self-confidence which, in turn, cause increased distress, anxiety and depression.

These findings add to the previous literature by suggesting that there is a relationship between hearing impairment and psychological outcomes and that it may be important to consider the role of individual as well as social characteristics. In particular, 
the presence of a hearing impairment may cause a decline in feelings of competence, control and self-efficacy which may be the strongest predictor of psychological health. These findings are consistent with other research which have found that sensory impairments predict significant reductions in perceived competence with basic activities of daily living (Marsiske et al., 1997). It is not known whether this finding can be generalized to other adults with communication impairments but some literature suggests that other conditions may function similarly in terms of their impact on perceived control, competence, and self-confidence (Babbitt \& Cherney, 2010; Baylor et al., 2011).

Summary. The extent to which communication impairments affect the social relationships of older adults is not well-understood. Communication impairments are associated with an increased risk of depression, social isolation, loneliness, and poorer quality of life and these findings have been confirmed in a variety of clinical populations. It is difficult to estimate the relative impact of a communication disorder, however, because they often co-occur with other health problems and functional limitations. Changes in social support and social network characteristics have also been observed in older adults with communication impairments but the importance of these measures for psychological well-being is not well-known. Some authors have reported that other variables, such as self-efficacy and mastery, may be more important for mental health. The relationship between communication status and more global measures, such as social participation, is also not well-understood. Evidence from pilot data for the current study suggested that communication impairments independently predicted levels of loneliness, social network size, frequency of positive social exchanges, and social participation in a 
sample of community-dwelling older adults. These findings require additional confirmation.

\section{Research Questions}

The proposed study seeks to better understand how communication impairments impact a diverse range of social relationship factors and the psychological well-being of community-dwelling older adults. The questions that will be addressed are as follows:

Question 1: Is there an association between communication impairment and social relationships? After controlling for demographic, health, and functional limitations, is communication impairment an independent predictor for social network size, social support, social participation, or negative social exchanges?

Hypothesis 1: The severity of communication impairment is an independent predictor of social network size. Consistent with previous research, it was hypothesized that the social networks of adults with more severe communication impairments will be smaller and also composed of a greater proportion of kin than non-kin. According to Socioemotional Selectivity Theory, decrease in the size of the social network is due to a voluntary process of selection (Carstensen, 1991). It was hypothesized, however, that the findings would not be consistent with Socioemotional Selectivity Theory, as it was also hypothesized that communication impairment severity will also be an independent predictor for loneliness. This would suggest that the current social network does not meet the needs for companionship of individuals with communication impairments and that older adults with communication impairments may not be able to negotiate their social needs and are less successful in "proactive aging" with regard to their social relationships (Lang \& Carstensen, 1994). The predicted findings, therefore, would be more consistent 
with a process of social exclusion or one of social withdrawal. Further evidence for this hypothesis would be sought during the qualitative interviews.

Hypothesis 2: The severity of a communication impairment is an independent predictor of reduced social support and reduced social participation. This finding would demonstrate that the hierarchical compensation model does not appear to describe the experience of older adults with communication impairments. More specifically, Cantor's $(1979,1980)$ contention that older adults are able to negotiate their support needs from closer or more distant social network members might not be applicable to older adults with communication impairments. Instead, the findings would be more consistent with Litwak's theory (1985) that particular relationships provide certain kinds of functions and, consequently, the loss of relationships may result in a loss of these functions.

Hypothesis 3: The severity of a communication impairment is not an independent predictor of negative social exchanges. There is a large body of literature showing that declines in communication have been shown to have negative impacts on intimate relationships including declines in marital intimacy and satisfaction, increased frustration and interpersonal strain, anger, resentment, and blame (Baikie, 2002; Carter, et al., 1998; Hétu et al., 1993; Joubert, et al., 2011). Consistent with Socioemotional Selectivity Theory, however, older adults seek to insulate themselves from relationships that are unrewarding or problematic. As a result, older adults who experience negative social exchanges may be able to limit their exposure to these types of interactions through social withdrawal (Morgan, 1989) which would result in less frequent negative social exchanges as well as a smaller social network size. This finding would be consistent with the 
findings from the preliminary study (Palmer et al., 2012) and also with Hypothesis 1. Further confirmation of this hypothesis would be sought during the qualitative interviews.

Question 2: What is the evidence for an interaction between communication impairment and physical disability in terms of their combined impact on psychological well-being? Specifically, what is the evidence that levels of physical disability moderate the relationship between communication impairment and either loneliness or depression, as illustrated in Figure 4?

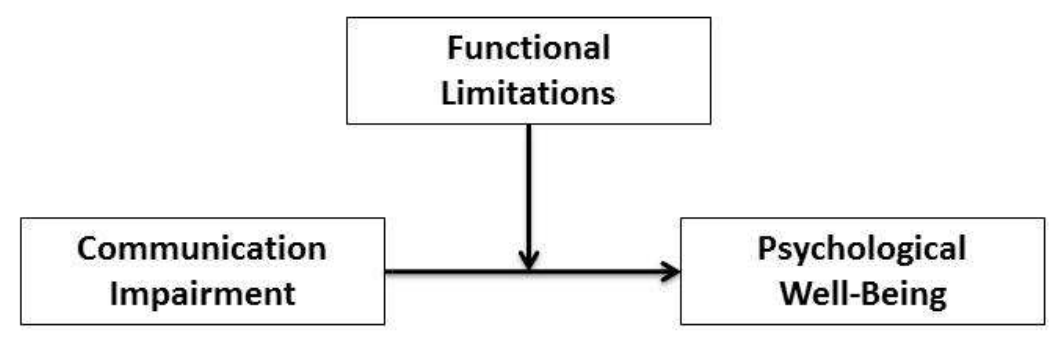

Figure 4. Hypothesized moderating effect of physical disability on psychological wellbeing.

Hypothesis 4: Functional limitations will moderate the relationship between communication impairments and mental health. Previous research has been equivocal with regard to the relationship between hearing impairments and well-being and little research has examined the psychological impact of communication impairment across multiple disorders. To date, only one study has examined a possible interaction between physical and sensory impairments (Kempen, Verbrugge, Merrill, \& Ormel, 1998). If there is an association between communication impairments and mental health only for those with higher levels of functional limitations, this finding would be consistent with theory of Person-Environment Fit, suggesting poorer "fit" between the individual and the 
environment. It would also be consistent with the theories of stress and coping and Selective Optimization and Coping, in that individuals with multiple disabilities may have reduced ability to compensate for their impairments leading to reduced self-efficacy, mastery and control, and a greater risk of psychological distress, loneliness, and depression as a result (Kramer et al., 2002; Marsiske et al., 1997; Ormel et al., 1997).

\section{Question 3: Does social support "buffer" the impact of a communication} impairment on psychological well-being? Specifically, is there evidence that social support might moderate the relationship between communication impairment and either loneliness or depression, as illustrated in Figure 5?

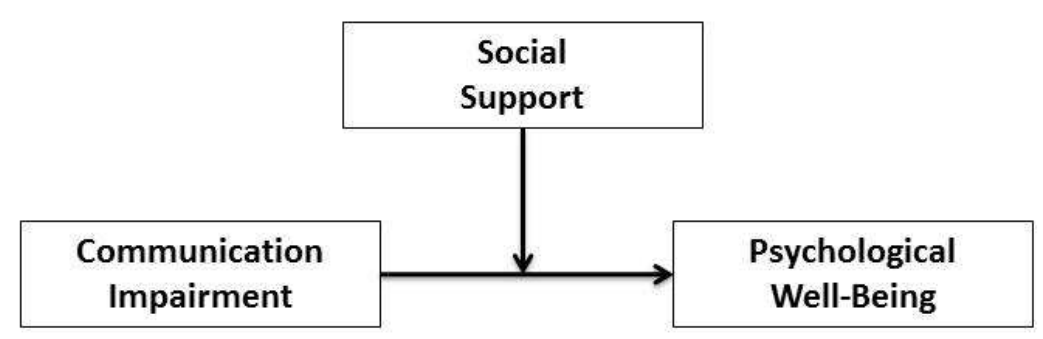

Figure 5. Hypothesized moderating effect of social support on psychological well-being.

Hypothesis 5: Social support will not moderate the relationship between communication impairment and either loneliness or depression. Previous research has been inconsistent with regard to the role of social support on psychological well-being in individuals with hearing impairment. Some studies have reported moderating role for social support (e.g., Frankel \& Turner, 1983) which would be consistent with stress and coping theories that conceptualize social support as a stress "buffer." In contrast, there have been studies that reported no such relationship (e.g., Oppegard et al., 1984), and other studies that reported an association but found the role of social support to be 
negligible when other factors were considered (e.g., Ormel et al., 1997). If social support does not moderate the relationship, this finding would be inconsistent with theories of stress and coping which emphasize social support as a strategy for reducing stress, but might suggest that the association between these variables should be conceptualized differently, as discussed below.

Question 4: Does the presence of a communication impairment cause a decline in social support or other aspects of social relationships which, in turn, leads to poorer psychological well-being? Specifically, is there evidence that social support or other characteristics of social relationships mediate the relationship between communication impairment and either loneliness or depression, as illustrated in Figure 6? There is some evidence that communication impairments may cause a decline in the quantity and quality of available support over time. In the longitudinal study by Pachana and colleagues (2008) of older Australian women, the presence of a hearing impairment was associated with a significant decrease in satisfaction with social support over time. The authors were not able to identify what had caused this decline in satisfaction but speculated that this could be due to an unwillingness or inability to participate in meaningful social interactions or a decreased ability to derive pleasure from these interactions. In theoretical terms, this finding could be consistent with Communication Accommodation Theory, in which failed support may occur at the dyadic level if the conversational partner either over- or under-accommodates the needs of an individual with communication impairment (Giles, Coupland, \& Coupland, 1991; McIntosh, 1996). This, in turn, might cause withdrawal from the support network (Morgan, 1989) placing the individual at increased risk for social isolation and negative well-being. 


\begin{tabular}{|c|c|c|}
\hline $\begin{array}{l}\text { Communication } \\
\text { Impairment }\end{array}$ & $\begin{array}{c}\text { Social } \\
\text { Relationships }\end{array}$ & $\begin{array}{c}\text { Psychological } \\
\text { Well-Being }\end{array}$ \\
\hline
\end{tabular}

Figure 6. Hypothesized mediating effect of social relationships on psychological wellbeing.

Hypothesis 6: Communication impairment causes a reduction in social network size and diversity, reduced opportunities to negotiate support, and reduced opportunities for social participation which, in turn, result in poorer psychological well-being. Research in this area has been limited to date but there is some evidence that there is a deterioration over time in the social support of individuals with hearing impairment (Pachana et al., 2008; Schneider et al., 2010). As discussed above, this is inconsistent with Socioemotional Selectivity Theory and the hierarchical compensation model. If this hypothesis was supported, the finding would support Litwak's view of the interrelationship between the loss of network members and the loss of the functions that they provide.

Question 5: Do individuals compensate for communication impairment by using relationship control strategies to negotiate access to a larger social network or access to more social support? What is the evidence that relationship control strategies moderate the relationship between communication impairment and social network size or levels of social support, as illustrated in Figure 7? As conceptualized by Bandura (1977), self-efficacy is the conviction that one can successfully execute a specific behavior in order to produce a particular outcome. Social self-efficacy has been defined as a belief in one's ability to deal effectively with others (Sherer et al., 1982). Some authors have 
argued that the effect of social relationships on well-being may be mediated by individual-level variables such as control (Antonucci, 2001). This has been described as the "support-efficacy model" in which it has been hypothesized that self-efficacy may help to explain the association between social relationships and well-being (Antonucci \& Jackson, 1987). Further, there is some evidence to suggest that the ability to manage social relationships and negotiate support needs may be more strongly predicted by domain-specific measures, such as social self-efficacy, rather than more general measures of control (Bisconti \& Bergeman, 1999). Although it has not been used widely in the aging literature, social self-efficacy has previously been shown to be a significant predictor of mental health in older adults (Fiori et al., 2006).

Hypothesis 7: Relationship control strategies do not moderate the relationship between communication impairment and social network size or levels of social support. Previous research has shown that communication impairments may lead to reduced selfefficacy, mastery and control (Kramer et al., 2002; Marsiske et al., 1997; Ormel et al., 1997). Consequently, it was predicted that individuals with more severe communication impairments will have lower social self-efficacy and that, as a result, they are not able to compensate for the presence of a communication impairment. This is consistent with previous research into the association with communication impairments and feelings of mastery, control, confidence, competence (Babbitt \& Cherney, 2010; Baylor et al., 2011; Marsiske et al., 1997). 


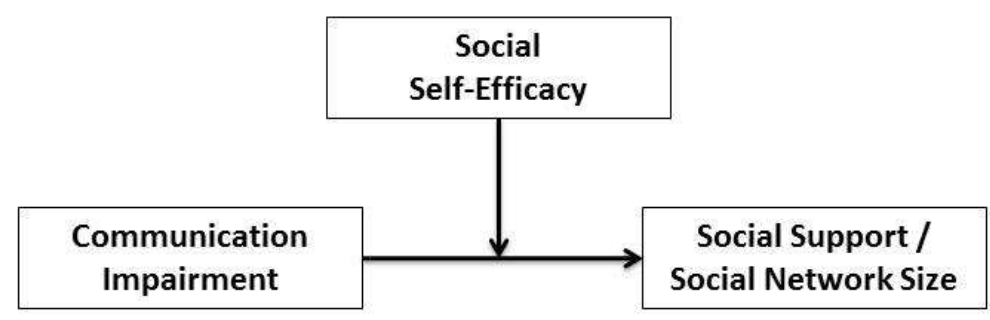

Figure 7. Hypothesized moderating effect of relationship control on social network size and social support.

Summary. A visual schematic of the interrelationships between variables in the previous questions is presented in Figure 8. The study variables have been placed in the context of the conceptual framework for the impact of social networks on health as outlined by Berkman and colleagues (2000; Figure 3). The role of individual characteristics were analyzed as predictors of each characteristic of social relationships. These, in turn, were examined as potential mediators or moderators of the relationship between communication impairments and psychological well-being. 


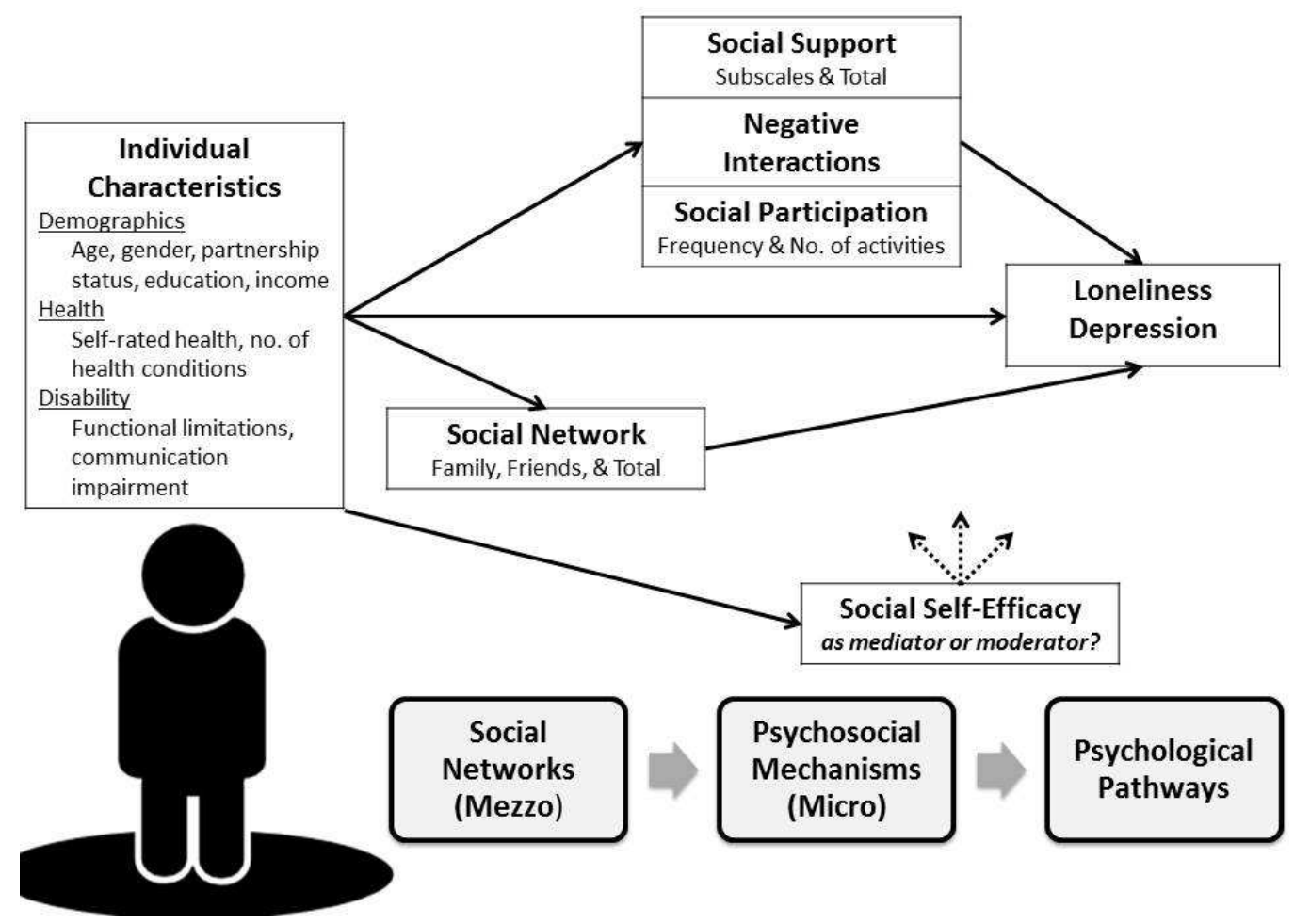

Figure 8. Summary schematic of the relationships between variables explored in the study. 


\section{Method}

\section{Study Design}

The goal of the current study was to provide detailed information about the health, communication status, and social relationships of a diverse sample of communitydwelling older adults. As described below, individuals with communication impairments due to a variety of etiologies were recruited for the study. In addition, older adults who had other medical or health problems but who had not been formally evaluated for a communication disorder were also included. The resulting study sample had a wide range of communication abilities, from those with negligible or very mild alterations in communication function to those who had more severe deficits. The study design also was intended to provide a sample of individuals who varied in their functional and physical abilities. The diagnoses associated with communication impairments were deliberately chosen to include those which may result in no physical limitations at all (e.g., hearing impairment) as well as those that may result in a variety of physical limitations (e.g., multiple sclerosis, Parkinson's Disease, and ALS). In addition, the adults who participated had other health conditions common to older adults (e.g., heart conditions, arthritis, diabetes) that can result in functional limitations. As with communication impairments, the purpose of the study design was to include participants with a diverse range of physical abilities. One of the limitations of previous research into the significance of communication impairments in community-dwelling older adults is that the incidence of communication impairments was low and the sample was also relatively healthy which may have limited the power of these studies (e.g., Cruice et al., 2005; Hickson et al., 1995; Lind et al., 2003). By gathering information on a sample with 
greater diversity, the study would be more likely to discover whether communication impairments have an independent effect on psychosocial outcomes after controlling for the relative impact of health and functional impairments.

Several aspects of the current study were novel, the choice of study population, the use of a single communication measure across a variety of disorders, and the use of a battery of well-validated social measures in individuals with communication impairments. With regard to the first aspect, a single patient population or a single type of deficit is typically studied in most communication disorders research, with very few exceptions. The limitations of this approach are that the findings from each study are limited to a specific subgroup or population and it is not known whether the findings can be generalized. By studying a wider variety of disorders, it might be possible to make a statement about the importance of communication for maintaining social relationships which could be generalized to older adults with communication impairments of varying etiologies. Studies across individuals with a variety of communication disorders are rare but demonstrate the fact that individuals experience many common difficulties (e.g., Baylor et al., 2011). Second, in studies of older adults that have examined how speech and hearing may impact social outcomes, objective measures have often been utilized (Cruice et al., 2005; Hickson et al., 1995; Lind et al., 2003). It is well-known, however, that objective measures are often poorly correlated with subjective measures of communication function (Wallhagen, 2002) and that even mild impairments (measured objectively) have a significant negative impact on psychosocial function (Mulrow, Aguilar, Endicott, Velez, et al., 1990; Scherer \& Frisina, 1998). Consequently for the current study a measure was used that examines "the role of communication in everyday 
settings" (Hickson et al., 1995, p.93). There are a number of well-validated communication measures that have been developed in recent years that are available for research purposes. To date, however, they have not typically been used in a sample with communication impairments of multiple etiologies. Consequently there is little information about how these measures perform across diagnoses. Third, measures that have been used to investigate social relationships have been used in a wide variety of studies of older adults but these measures have not been used in older adults with communication impairments other than hearing impairment. For all three of these reasons, it was possible that there might be questions about the validity of the findings. As a result, a mixed methods design was selected for this study in order to provide additional methodological rigor.

Mixed methods research involves the collection or analysis of both quantitative and qualitative data in a single study in which data may be collected either concurrently or sequentially, analyzed separately, and ultimately integrated into a single set of findings (Cresswell, Plano Clark, Gutmann, \& Hanson, 2003). The combination of qualitative and quantitative methods is of particular value in studies of social phenomena that must take many different factors into account in studying these complexities (Greene \& Caracelli, 1997). A mixed-methods design may also provide stronger inferences, because it also allows for the presentation of a greater diversity of views and may also provide additional and alternative explanations for the study findings than those initially hypothesized by the investigators (Teddlie \& Tashakkori, 2003). By supplementing the survey data collected from a larger sample with more detailed explanatory information from a smaller subsample of participants, additional validity can be provided for the study findings and 
conclusions. In combining quantitative and qualitative methods, researchers typically assign either priority or a sequence to the use of the methods in order to facilitate the integration of findings (Morgan, 1998). According to the terminology which has become widely accepted with regard to the Priority-Sequence Model, the current study used a "QUANT => qual" or "Qualitative Follow-Up" design in which a smaller qualitative study was used to evaluate and interpret results from a primarily quantitative study.

There were two phases to the present study. A quantitative survey from a larger group of study participants was followed by an in-depth qualitative interview for a smaller, selected subsample. The goal for recruitment for the quantitative portion of the study was 100 participants, as discussed in the sample size and power section. The larger sample was supplemented by qualitative data from a subsample of 14 selected participants selected to explore and illustrate the findings from Phase 1. A summary of the recruitment methods for Phase 1 and Phase 2 of the study is shown in Table 3.

Table 3

A Summary of Recruitment Methods for Phases 1 and 2

\begin{tabular}{lll}
\hline Phase & \multicolumn{1}{c}{ Purpose } & \multicolumn{1}{c}{ Methods } \\
\hline $\begin{array}{l}\text { Phase 1 } \\
\text { (Quant) }\end{array}$ & Targeted recruitment & $\begin{array}{l}\text { 300 older adults seen at Oregon Health \& Science } \\
\text { University who met study criteria were targeted by } \\
\text { mail and follow-up postcard reminders for } \\
\text { participation. }\end{array}$ \\
& Non-targeted recruitment & $\begin{array}{l}\text { Invitations to participate in the study were posted on } \\
\text { appropriate internet support group message boards. } \\
\text { Email messages and invitations were distributed to } \\
\text { support groups in the Pacific Northwest as well as } \\
\text { speech and hearing professionals nationally. }\end{array}$ \\
& & $\begin{array}{l}\text { Information about the study was posted on OHSU's } \\
\text { Research Opportunities page and also distributed to } \\
\text { potential volunteers via ResearchMatch. }\end{array}$ \\
& & 14 individuals from the targeted recruitment were \\
Phase 2 & $\begin{array}{l}\text { Selection of individuals for } \\
\text { (Qual) } \\
\text { qualitative interviews }\end{array}$ & contacted for individual face-to-face interviews. \\
\hline
\end{tabular}




\section{Phase 1 Participants}

Data was collected and pooled from older adults including: a) those who had recently received evaluation or treatment by a speech-language pathologist or audiologist; and, b) those who had not been evaluated. The assumption was that the resulting study sample would have a range of communication abilities, ranging from those with negligible or very mild alterations in communication function which they have been easily able to accommodate to those who have more severe deficits. The data on these individuals was collected by the recruitment strategies described below. Based on a power analysis, it was determined that a minimum sample of 100 participants would be adequate to perform the analyses described in this chapter. To ensure a sufficient number of respondents, given the typically low rates of response to survey research, a total of 300 individuals were directly targeted. The targeted recruitment of these individuals was also supplemented by non-targeted recruitment through a variety of means to increase the likelihood of obtaining an adequate response from a diverse sample, as summarized in Table 3.

Targeted mailing of selected older adults. A targeted mailing of communitydwelling older adults who met the study criteria was conducted. Participants were patients or former patients at Oregon Health and Science University's (OHSU) Department of Otolaryngology during the previous year. Eligibility criteria included that all individuals had previously seen a physician at OHSU. This allowed review of their medical history to ensure that potential respondents met the study criteria. Following approval of the study protocol by both OHSU's Institutional Review Board (IRB) and 
Portland State University's (PSU) Human Subjects Research Review Committee (HSRRC; Appendix G) potential participants were identified using a search of OHSU's electronic medical record system. These individuals were mailed the survey questionnaire, a cover letter, an IRB-approved Information Sheet containing more information about the study (Appendix H), and a stamped addressed envelope with instructions to return the survey by mail by a deadline. Information about completing the survey online was also included. Postcard reminders were sent to all participants to encourage participation prior to the deadline.

Older adults with diagnosed communication impairments. This group of survey participants included community-dwelling older adults (65 or older) who had a communication impairment that originated after reaching adulthood. Participants were patients or former patients at OHSU. The presence of a communication impairment was established by the fact that the individual had sought speech pathology or audiology services for evaluation or treatment within the previous year. Potential participants were identified using a search of OHSU's electronic medical record system. Targeted participants were identified by generating reports of individuals previously seen at OHSU's Department of Otolaryngology. During the 14-month period from January 1, 2013 to March 31, 2014, a total of 1,886 patients aged 65 or older were seen by the speech pathology and audiology departments of OHSU's Department of Otolaryngology.

The study included individuals with a communication impairment resulting from each of the following four categories: benign voice disorders (e.g., vocal tremor, vocal fold paresis/paralysis, benign masses, edema, bowing, spasmodic dysphonia, \& muscle tension dysphonia); neurologic disease (e.g., stroke, ALS, MS, and PD); surgical or non- 
surgical treatment of head and neck cancer; and, hearing impairment. A total of 200 individuals were targeted by this means, fifty in each group. Diagnosis codes from Centers for Disease Control and Prevention's clinical modification of the ninth revision of the World Health Organization's International Classification of Diseases coding system (ICD-9) were being used at this time. Of the 1,886 individuals seen during this time, 1,193 had ICD-9 diagnosis codes consistent with one of the four categories. For more detailed information, see Appendix I, Table I1.

To be eligible, the onset of communication impairment must have occurred in adulthood and could not have been of developmental origin. The reason for this requirement was to ensure that all participants have the opportunity to reach adulthood and develop a normal range of social relationships. Because most of the study measures were based on self-report, individuals with diagnoses noted to be frequently associated with cognitive impairments (including Alzheimer's Disease/dementia, head injury/traumatic brain injury, and Huntington's Disease) or who had documented cognitive impairments (e.g. mild cognitive impairment) were excluded. In addition, individuals who had been referred for or had undergone cognitive testing according to their medical record at OHSU were also excluded. As most of the neurologic diagnoses in the study are known to be associated with changes in cognition, and because of the common co-occurrence of hearing and cognitive changes, some cognitive changes are to be anticipated in the study sample. To control for this, responses from individuals who report problems remembering "all of the time" or "almost everything" on the Washington Group's Extended Question Set on Functioning (2011; Appendix B) were also excluded. 
Cancer patients were targeted for inclusion in the study. Given the disruption frequently caused by cancer treatment, only medically stable individuals were recruited. This was defined as being currently cancer-free, not currently undergoing cancer treatment, and having completed treatment at least one year previously to allow for adequate recuperation and rehabilitation from the acute side-effects of treatment. To ensure that all participants are potentially able to participate in face-to-face interviews, individuals currently living outside the Pacific Northwest were also excluded. In summary, the full list of inclusion criteria for this group was as follows:

1. Being aged 65 or more.

2. Having been seen by a physician and a speech pathologist or audiologist in the Department of Otolaryngology at OHSU in the previous year.

3. Being a native English speaker.

4. Living independently in the community in the Pacific Northwest region.

5. Not having any conditions known to cause significant cognitive impairment.

6. Being cancer free currently or having completed cancer treatment at least a year previously.

7. Having a communication impairment which began in adulthood.

Once subdivided into the four categories, the names of the individuals on each list were assigned a random number, reordered numerically, and then reviewed sequentially until a total of 50 eligible individuals had been identified. A total of 347 charts were reviewed in order to identify 200 individuals who met eligibility criteria. The total number of charts reviewed for each of the four groups was as follows: benign voice disorders, $(n=67)$; neurologic conditions, $(n=91)$; head and neck cancer, $(n=115)$; 
and, hearing impairment, $(n=74)$. The reasons for exclusion of the 147 other individuals are listed in Appendix I, Table I2.

Older adults without diagnosed communication impairments. To supplement the data from those with a diagnosed communication impairment, one hundred older adults without any of the above diagnoses were also contacted for participation. They were identified by generating a list of all older adults that have been seen at OHSU in the Department of Otolaryngology in the previous year. Individuals were excluded if they had any medical diagnosis known to cause significant changes in cognition (e.g. Alzheimer's disease/dementia) even if they had not undergone medical work-up with regard to that condition.

It was assumed that the individuals on this list would have a wide range of medical conditions common in the aging population (e.g., arthritis, cardiac conditions, diabetes, thyroid disorders) and would therefore be expected to havechanges in communication and a variety of physical health and functional limitations associated with typical aging. Individuals were excluded if they had a history of communication impairments, as demonstrated by having been seen by speech pathology or audiology services previously either at OHSU or elsewhere, or if they had any types of communication difficulties documented in their medical record. The full list of inclusion criteria for this group was as follows:

1. Being aged 65 or more.

2. Having been seen by a physician in the Department of Otolaryngology at OHSU in the previous year.

3. Being a native English speaker. 
4. Living independently in the community in the Pacific Northwest region.

5. Not having any conditions known to cause significant cognitive impairment.

6. Being cancer free currently and, if having been treated for cancer in the past, having completed cancer treatment at least a year previously.

7. Not having been seen by a speech pathologist or audiologist at OHSU or elsewhere or having a documented communication impairment.

During the same period, a total of 3,252 patients aged 65 or older were seen by physicians in OHSU's Department of Otolaryngology. After the removal of all those who had been seen by speech pathology and audiology during the same time period, a total of 2,139 individuals remained. These individuals were then assigned a random number, reordered numerically, and then reviewed sequentially until a total of 100 patients meeting eligibility criteria were identified. A total of 235 charts were reviewed before 100 eligible participants were found. The specific reasons for exclusion of the 135 individuals are listed in Appendix I, Table I3.

Non-targeted recruitment. To supplement the data from the targeted mailing and generate a sample of adequate size for subsequent analysis, a more general recruitment strategy was also employed. This included recruiting individuals with the some of the same types of communication impairments and conditions listed above as well as older adults with a variety of other medical conditions and no history of communication difficulties. Potential participants could complete the survey either over the internet or in paper form by mail. The advantage of this strategy was that it allowed generation of a larger number of responses economically. The disadvantage was that potential participants had not been prescreened for eligibility. Consequently individuals 
were asked to complete questions about difficulties with memory or concentration using a self-report screening questionnaire prior to participation and, as above, data from individuals who reported more than mild difficulties with memory or concentration were excluded.

During September and October 2014, general recruitment of non-targeted participants occurred by a variety of means. Information about the study was posted on OHSU's Research Opportunities page and through ResearchMatch, an online service that allows researchers to identify volunteers around the country that meet particular study criteria. In addition, messages were posted on list-serves for professionals at speech and hearing clinics nationally. Information about the study was posted for speech pathologists and audiologists who belong to one of several of the American Speech-Language Hearing Association's online forums and Special Interest Groups, namely the Healthcare, Hearing, and Research forums, as well as Special Interest Groups for Voice, Hearing, and Gerontology. With the approval of the administrators, messages about the study were posted on online on support group forums for individuals with a wide variety of medical conditions including ALS (www.alsforums.com), hearing loss (www.myhearingloss.org), head and neck cancer (www.webwhispers.org), and spasmodic dysphonia (http://www.dysphonia-bb.org/forums/sd/), as well as general message boards for older adults (www.dailystrength.org/support-groups/Seniors). In addition, information about the study was posted online on the National MS Society's website and a tweet about the study was also sent to members of the National Association for the Deaf.

Study response for targeted participants. The initial mailing occurred in August 2014. The mailing consisted of a cover letter, an information sheet with more 
information about the study, and a copy of the study survey. A subsequent mailed reminder was also sent to those individuals who had not responded in September. By the response deadline of 10/31/14, a total of 145 targeted individuals had returned completed the survey, representing an overall response rate of $48 \%$. Response rates by group ranged from $44-62 \%$, as summarized in Table 4 . Of those targeted who did not participate, four individuals declined, one individual was deceased, and one survey was returned marked "undeliverable."

Of the targeted participants who completed the survey, twelve individuals were excluded for the following reasons: the survey being largely incomplete $(n=1)$; difficulty remembering "all of the time" $(n=1)$; difficulty remembering "almost everything" $(n=$ 1); history of traumatic brain injury $(n=3)$; history of childhood communication disorder (stuttering, $n=3$; hearing loss, $n=1$; cerebral palsy, $n=1$ ); and, one individual was excluded for more than one reason (history of traumatic brain injury and history of stuttering). As a result, the final number of individuals who completed the survey and were eligible for inclusion was 133.

Table 4

Response Rate by Group for Targeted Participants

\begin{tabular}{lcc}
\hline \multicolumn{1}{c}{ Group } & $\mathrm{n}$ & $\%$ \\
\hline A. Benign voice disorders & 31 & 62.00 \\
B. Neurologic conditions & 24 & 48.00 \\
C. Head and neck cancer & 23 & 46.00 \\
D. Hearing impairment & 23 & 46.00 \\
E. General Otolaryngology & 44 & 44.00 \\
\hline All groups & 145 & 48.33 \\
\hline
\end{tabular}


Study response for non-targeted participants. A total of 128 non-targeted individuals completed the survey, 124 online and 4 by mail. Of these a total of fourteen did not pass the initial online screening questions in order to participate in the study due to: being under $65(n=4)$; not living independently in the community $(n=1)$; having trouble remembering or concentrating most or all of the time $(n=3)$; having had a childhood communication disorder $(n=6)$; being currently treated for cancer $(n=1)$; or having been treated for cancer in the last year $(n=4)$. These categories were not mutually exclusive and five of the fourteen individuals were excluded for more than one reason.

Of the 114 individuals who submitted the survey, an additional 7 individuals were excluded for the following reasons: history of traumatic brain injury $(n=1)$; history of childhood communication disorder (stuttering, $n=2$ ); living outside North America (Australia, $n=1)$; self-reported age under $65(n=1)$; and the survey being largely incomplete $(n=2)$. As a result, the final number of individuals who completed the online survey and were eligible for inclusion was 107 . The final study sample by group is summarized in Table 5.

Table 5

Number of Responses Included in the Final Sample by Group for All Participants

\begin{tabular}{lc}
\hline \multicolumn{1}{c}{ Group } & $\mathrm{n}$ \\
\hline A. Benign voice disorders & 28 \\
B. Neurologic conditions & 21 \\
C. Head and neck cancer & 23 \\
D. Hearing impairment & 22 \\
E. General Otolaryngology & 39 \\
F. Volunteers & 107 \\
\hline All groups & 240 \\
\hline
\end{tabular}




\section{Phase 1 Measures}

Previously validated measures and instruments were used in the study to enhance the strength of the findings. These were supplemented by open-ended qualitative questions, as described in the subsequent sections. A summary of all of the published instruments is listed in Appendix A and the individual published instruments are included in Appendices B to D.

Sociodemographic variables. Sociodemographic variables included standard assessments of age, gender, marital status, education, and annual household income, as used in other national surveys of older adults, such as the Later Life Study of Social Exchanges (LLSSE; Sorkin \& Rook, 2004) and the National Social Life, Health, and Aging Project (Waite et al., 2007). As covariates in the proposed analyses, the following sociodemographic factors were used: age (continuous); gender $(0=$ male, $1=$ female $)$; partnership status $(0=$ single/never $\mathrm{married} / \mathrm{separated} / \mathrm{divorced} /$ widowed, $1=$ married or living with partner); highest level of education completed $(0=$ high school or less, $1=$ associate/trade or vocational/some college, 2 = four-year college degree or more); annual household income $(0=<\$ 25,000,1=\$ 25-50,000,2=\$ 50-75,000,3=\$ 75-100,000,4=$ $>\$ 100,000)$

Self-rated health. Self-rated health was measured using the commonly used single item: "How would you describe your health at the present time? Would you say it is excellent, very good, good, fair or poor?" $(0=$ poor, $4=$ excellent; Ware \& Sherbourne, 1992).

Comorbidity. The number of health conditions was assessed by asking participants, "Have you ever been told by a doctor or other health professional that you 
have..." any of 12 common conditions (i.e., high blood pressure/hypertension, asthma, emphysema/chronic bronchitis, arthritis/rheumatism, diabetes, stomach/intestinal ulcers, liver disease, kidney/bladder problems, cancer, heart attack/heart failure, stroke, hip fracture, and other). The presence or absence of each condition was scored in a binary fashion $(1=$ yes, $0=$ no) as in other surveys (Manton, Stallard, \& Corder, 1998; Sorkin \& Rook, 2004). A total was created by summing the total number of conditions present. For descriptive purposes, a list of other conditions relevant to the study was also included and participants were asked to list "other" conditions not included in the list.

Functional limitations. Functional limitations were measured with 15 questions that included activities of daily living (ADLs, e.g. bathing; Katz, Ford, Moskowitz, Jackson, \& Jaffe, 1963), instrumental activities of daily living (IADLs, e.g. preparing own meals; Lawton \& Brody, 1969), upper extremity strength (e.g. grasping objects; Nagi, 1976), and mobility (e.g. climbing stairs; Rosow \& Breslau, 1966). A five point scale was used $(0=$ can't do at all, $5=$ not at all difficult). For purposes of comparison with data from the LLSSE study scores was recoded into a four-point scale $(0=$ not at all difficult, 3 = very difficult/unable to do) with higher scores indicating greater difficulty. Fourteen of these items were then be averaged to create an overall measure of functional impairment. One instrumental activity of daily living item (telephone use) was excluded from the calculation, as this relates to communication. Previously, this 14-item version of the measure showed excellent internal reliability in a community dwelling sample of older adults, $\alpha=.92$ (Palmer et al., 2012).

Communication. The Communicative Effectiveness Index is a 16-item survey originally developed as a measure of functional communication for individuals with 
aphasia (Lomas et al., 1989). More recently the original 16-item version of the scale was revised into a 10-item version that focuses on social communication, the Communicative Effectiveness Index-Modified (CETI-M; Yorkston et al., 1999; Appendix B). In a study of the use of the modified version in 25 individuals with ALS and their partners, internal reliability for the CETI-M was excellent, $r=.97$, and no significant differences were found in the relative ranking of the 10-items of the scale in terms of their relative difficulty between individuals with ALS and their partners (Ball, Beukelman, \& Pattee, 2004). The correlation between individuals with ALS and their partners for the total score was very consistent, $r=.87$. In addition, a significant non-linear correlation was observed between self-reported measures of communicative participation and objective testing of speech intelligibility, $r=.95$. Since that time, the CETI-M has been used in studies of individuals with ALS, Parkinson Disease, and other speech impairments (Halpern et al., 2012; Joubert et al., 2011; Judge, Clarke \& Hawley, 2011). Although it has not been used in other populations, content validity for this measure is supported by the fact that it shares a number of content items with other widely-used communication measures, including the Communicative Effectiveness Survey (Donovan, Kendall, Young, \& Rosenbek, 2008), Hearing Handicap Inventory for the Elderly Screening Version (Ventry \& Weinstein, 1982), and Voice Handicap Index-Functional subscale (Jacobson et al., 1997). A comparison of the content of these scales is listed in Appendix E. In regression analyses the mean score on the CETI-M was used as a continuous variable.

Psychological well-being. Two measures were used to assess different aspects of psychological well-being, as described below. These instruments are included in Appendix C. 
Loneliness. The UCLA Loneliness Scale was originally published in 1978 and has subsequently undergone two revisions (Russell, 1996). It is a 20-item self-rated scale in which respondents indicate how often they experience feelings of loneliness and social isolation. The original scale was shown to have high internal reliability, $\alpha=.96$, validity, and test-retest reliability, $r=.73$. It is the most commonly used self-report loneliness instrument for both clinicians and researchers and has been widely used with older adults (Dickens, Richards, Greaves \& Campbell, 2011; Luanaigh \& Lawlor, 2008). The measure has also had some limited use in research relating to individuals with communication impairments (Philp, Lowles, Armstrong \& Whitehead, 2002; Poissant et al., 2008). In the LLSSE study (Sorkin \& Rook, 2004), loneliness was assessed using an abbreviated 6-item version of the UCLA Loneliness Scale. The internal reliability of the 6-item version was .71 at Wave 1. The same 6-item version of the UCLA Loneliness Scale was used in the current study.

Depression. Of the many screening and measurement tools which have been used to identify depressive symptoms in adults, the most widely-used is the Center for Epidemiologic Studies-Depression Scale (CES-D; Radloff, 1977). A revised 9-item version of the CES-D has been shown to be an efficient method of screening for depression (Santor \& Coyne, 1997). The revised version has good internal consistency and is strongly correlated with the total score of the original, $r=.93$. In a review of 42 published studies of older adults, however, the CES-D was found to be equally accurate (and sometimes more accurate) in identifying symptoms of depression in older adults than other scales developed specifically for this population (Wancata, Alexandrowicz, Marquart, Weiss, \& Friedrich, 2006). 
Measures of social relationships. Four different aspects of social relationships were assessed using a variety of validated measures in order to assess social network size and composition, social support, social participation, and the frequency of negative social interactions. These instruments are described next and the complete instruments are included in Appendix D.

Social network size and composition. The Lubben Social Network Scale (LSNS; Lubben, 1988) is an instrument designed to gauge social isolation in older adults and was originally developed as an adaptation of the Berkman-Syme Social Network Index. Since its publication the LSNS has been used in more than a hundred research studies (Lubben \& Gironda, 2004). More recently the LSNS was revised to address four methodological issues with the original scale (LSNS-R; Lubben \& Gironda, 2004). The LSNS-R consists of 12 items, half of which relate to family relationships and half of which relate to relationships with friends (Lubben, Gironda, \& Lee, 2002). Compared to the original LSNS, the use of the LSNS-R in a sample of older adults was shown to increase the internal consistency of the scale from a Cronbach's alpha of .66 to .78 (Lubben \& Gironda, 2004). In a recent study of community-dwelling older adults (Wells, 2009), the internal reliability for the total LSNS-R was excellent, $\alpha=.90$, and also for the friends and family subscales individually, $\alpha=.89$ and .88 , respectively.

Social support. Social support was measured using the Social Provisions Scale (SPS; Cutrona \& Russell, 1987), a 24-item scale of perceived social support based on Robert Weiss's social provisions model (1974). Respondents are asked to rate the how strongly they agree or disagree with a series of statements using a four-point scale $(1=$ strongly disagree, 4 = strongly agree). Of the 24 items, four are devoted to each of the six 
separate social provisions. A combined score for each of the six subscales is calculated as well as a total score. The SPS has been shown to be a reliable and valid instrument of use in older populations (Cutrona \& Russell, 1987; Mancini \& Blieszner, 1992). Internal consistency for the total scale is reported to be from .85 to .92 across a variety of populations and alpha coefficients for the various subscales range from .64 to .76 (Cutrona, 1986). The SPS has been used in multiple studies of social support in older adults and has been shown to be a predictor of mental and physical health outcomes in the elderly and to be associated with objective network characteristics (Cutrona, 1986; Cutrona, Russell \& Rose, 1986; Felton \& Berry, 1992; Russell \& Cutrona, 1991). Its use in individuals with disability is more limited but it has been shown to be a valid measure for assessing the interaction between social support, well-being, and adaptation in one study of visually impaired adults (McIlvane \& Reinhardt, 2001).

Negative social exchanges. The frequency of negative social exchanges was assessed using the Negative Interaction Scale (NIS; Krause, 1995). The NIS asks the respondent to report the frequency of overly demanding behavior, criticism, intrusiveness, and being taken advantage of over the previous month, using a four point scale $(0=$ never, $3=$ very often $)$. A total score was calculated by summing the four items. The scale has been used previously in research with older adults and has been shown to be associated with satisfaction with social support. In addition, the measure has strong internal consistency and test-retest reliability (Krause \& Shaw, 2002).

Social participation. The frequency of participation in recreational and social activities was assessed using items from the Social Disengagement Index (Bassuk et al., 1999) as previously adapted for use with older adults (Sorkin \& Rook, 2004). 
Respondents were asked to report their frequency of participation in ten different types of activities over the course of the previous month $(0=$ never or almost never, $5=$ daily $)$. A combined frequency of social participation score was be created by averaging the ten items. In addition the total number of social activities was calculated by summing the number of activities participated in at least once during the previous month. Previously, internal consistency for the scale was shown to be acceptable, $\alpha=.63$ (Palmer et al., 2012).

Measures of relationship control at the individual level. The extent to which activity at the individual level is responsible for maintaining either the size of the social network and the types of social provisions that it provides access to is not well-known. This type of activity would be consistent with theories such as Socioemotional Selectivity Theory, Selective Optimization with Compensation and the "hierarchical compensation model" (Baltes, 1987, 1997; Cantor, 1979, 1980; Carstensen et al., 1999). These theories emphasize that older adults may be able to compensate for relationship losses by actively negotiating ways to meet their practical and emotional needs. It is not known to what extent these processes may explain changes in the social relationships of older adults with communication impairments. To test the extent to which activity at the individual level may be at work, a measure of social self-efficacy was used.

Social self-efficacy. As conceptualized by Bandura (1977), self-efficacy is the conviction that one can successfully execute a specific behavior in order to produce a particular outcome. During the development of the Self-Efficacy Scale (Sherer et al., 1982), factor analysis revealed two domains of self-efficacy which resulted in the creation of a General Self-Efficacy and a Social Self-Efficacy subscale. The latter 
consists of 6 items relating to efficacy expectations in social situations (e.g. "I do not handle myself well in social gatherings.”) Cronbach's alpha for the social subscale was .71. Scores on both scales were associated with but substantially different from other measures of personality, including locus of control, personal control, social desirability, ego strength, interpersonal competence, and self-esteem, which provided evidence of construct validity. Social self-efficacy has been defined as a belief in one's ability to deal effectively with others (Sherer et al., 1982). Some authors have argued that the relationship between social relationships and well-being may be mediated by individuallevel variables such as control (Antonucci, 2001). Further, evidence suggests that this association may be more strongly predicted by domain-specific measures such as social self-efficacy (Bisconti \& Bergeman, 1999). This has been described as the "supportefficacy model" in which it has been hypothesized that self-efficacy may help to explain the association between social relationships and well-being (Antonucci \& Jackson, 1987). Although it has not been used widely in the aging literature, this measure has previously been shown to be a significant predictor of mental health in older adults. Katherine Fiori and her colleagues (2006) found social self-efficacy to be a partial mediator of the relationship between social relationships and depressive symptoms in a sample of older adults. Interestingly, this relationship was not true of middle-aged adults, which raises the possibility that this domain may become increasingly important with age. In addition to the validated measure, two open-ended questions were included in the survey to gather more information regarding: a) how these characteristics of social self-efficacy have changed over time; and, b) the reasons for these changes. 


\section{Phase 1 Analysis}

Sample size and power. The study was powered to address the primary objective, namely whether communication impairment severity is an independent predictor of a range of psychosocial outcomes in a multiple regression analysis after controlling for the study covariates. To achieve a power of $80 \%$ (i.e., $20 \%$ chance of accepting the null hypothesis in error) with a significance level of .05 , sample size was calculated for a small, medium, and large effect size as defined by J. Cohen (1988) with a single predictor and 10 covariates. Covariates were assumed to account for $15 \%$ of the variance in the model. Sample sizes for three effect sizes are listed in Table 6, below. In a previous analysis (Palmer et al., 2012) communication impairment had an unstandardized beta ranging from .04 to .13 with regard to seven psychosocial outcomes of interest, suggesting an effect size ranging from small to medium. The cumulative $\mathrm{R}^{2}$ for the model as a whole ranged from .05 to .21 . As a result it was estimated that a sample of approximately 100 participants would be sufficient to address the primary research question.

Table 6

Calculated Sample Sizes for Various Effect Sizes in the Final Regression Model

\begin{tabular}{ccc}
\hline Effect size & Increment in $\mathbf{R}^{\mathbf{2}}$ & Minimum sample size \\
\hline Small & 0.01 & 98 \\
Medium & 0.09 & 65 \\
Large & 0.25 & 38 \\
\hline
\end{tabular}

Descriptive statistics. Descriptive data for all participants were summarized for background characteristics and all study variables, including mean, standard deviation, minimum and maximum values. 
Reliability analyses. Reliability analyses were performed for all study variables, including visual inspection of the data, identification of outliers, and calculations of the shape of distributions and internal consistency. After calculating means, medians, standard deviations, and ranges for all continuous variables a box plot of the data was generated. Outliers were identified and the original data re-examined for errors in calculations or data-entry. An internal reliability analysis for all scales was conducted by obtaining a Cronbach's alpha. Alpha values of .7 or more are generally considered acceptable. Skewness measures the degree to which a distribution of values is not symmetrical around the mean. Kurtosis values were used to assess departures from normal distributions in terms of the peak and tail weight of a given distribution. Robust alternative analyses were considered if distributions of the dependent variable were extreme. Options included transformation of the variable in question or use of alternatives to ordinary least squares in the regression analysis, such as least absolute deviation, least-trimmed squares, or M-estimation.

Inferential statistics. Initial analyses included bivariate correlations among all variables to investigate the first-order relationships. Comparisons were also made to determine if study measures varied significantly between participant groups. Research questions were investigated using simultaneous ordinary least squares multiple regression. Analyses were performed using SPSS, version 21 (IBM Corp., 2014). Each model included the eight health and demographic covariates listed above, namely age, gender, partnership status, education, household income, self-rated health, number of health conditions, and functional limitations as covariates. To ensure that multicollinearity was not present in the model, variance inflation factors were inspected 
to determine if values were above 10 which would indicate problematic levels of multicollinearity (Cohen, Cohen, West \& Aiken, 2003). Tests for mediation were conducted using the INDIRECT SPSS macro for multiple mediation by Preacher and Hayes (2008). Tests for simple slopes were conducted using the SIMPLE SPSS macro by O'Connor (1988). Analysis for each of the study questions was conducted as follows.

Question 1: Is there an association between communication impairment and social relationships? After controlling for demographic, health, and functional limitations, is communication impairment an independent predictor for any of the following: a) social network size; b) social support; c) social participation; or, d) negative social exchanges. A total of twelve multiple regression analyses were conducted. Communication impairment severity as measured by the total score on the CETI-M was entered in the model as the primary predictor. The model also included the eight following covariates: age, gender, partnership status, education, household income, selfrated health, number of health conditions, and functional limitations. Multiple regressions were performed with social network size (for both family and friends), social support (including all six subtypes), social participation, and the frequency of negative interactions as the outcomes of interest.

Question 2: What is the evidence for an interaction between communication impairment and physical disability in terms of their combined impact on psychological well-being? To answer this question (illustrated in Figure 4), two multiple regressions were tested with loneliness and depression as the outcomes of interest and communication impairment as a predictor. The model also included the seven following 
covariates: age, gender, partnership status, education, household income, self-rated health, and number of health conditions.

\section{Question 3: Does social support "buffer" the impact of a communication} impairment on psychological well-being? To answer this question (illustrated in Figure 5), multiple regressions were conducted with depression and loneliness as the outcomes of interest and communication impairment as a predictor. The model also included the same eight covariates as in Question 1.

Question 4: Does the presence of a communication impairment cause a decline in social support or other aspects of social relationships which, in turn, leads to poorer psychological well-being? To answer this question (illustrated in Figure 6), multiple regressions were tested with depression and loneliness as the outcomes of interest. Each of the social relationships found to be significantly associated with communication impairment in Question 1 were tested individually and then all significant mediators were tested in a multiple mediation model. The model also included the same eight covariates as in Question 1.

Question 5: Are individuals able to compensate for communication impairment by using relationship control strategies to negotiate access to a larger social network or access to more social support? To answer this question (illustrated in Figure 7), two multiple regressions will be conducted with social network size and social support as the outcomes of interest and communication impairment as a predictor. The model also included the same eight covariates as in Question 1. 


\section{Phase 2 Qualitative Analysis Plan}

Sampling for Phase 2. Purposive sampling (Cresswell, 2013) was used for the targeted recruitment of individuals to ensure that an adequately diverse sample. The quantitative data from Phase 1 was used to select a subsample of 14 individuals for indepth qualitative interviews designed to gather more detailed information about the social lives of the participants, how they might have changed over time, the reasons for those changes, and their impact. This number of interviews is considered adequate for phenomenological studies (Polkinghorne, 1989). Efforts were made to ensure that the interview participants were sufficiently varied with regard to their age, gender, type of communication impairment, and health, to ensure representativeness. Selecting individuals in this manner helped to ensure a) an adequate diversity of opinion and, b) insight into the different factors that may be related to differences in outcomes. The location of these interviews was chosen by the participant with the option of being performed in their home or at OHSU, depending on individual preference. Interviews were anticipated to last approximately an hour and were audio-recorded for subsequent transcription and analysis.

Open-ended communication questions. Qualitative information was gathered using an open-ended questioning technique in the manner recommended by Cresswell (2007), following the procedures for performing phenomenological research. The purpose of a phenomenological approach is to describe the meaning for multiple individuals of "their lived experiences of a concept or phenomenon" and to develop an understanding of the commonalities in their experience that can be used to understand "the very nature of the thing" (p.58). The open-ended questions to be used during the 
individual interviews are listed in Appendix F. Polkinghorne (1989) recommends that researchers interview between 5 and 25 individuals who have experienced the phenomenon in question for phenomenological studies

Analysis of qualitative responses. In analyzing the qualitative data generated during the individual interviews a phenomenological method was employed, in keeping with previously described methods (Cresswell, 2007; Moustakas, 1994). All of the responses from study participants were recorded during the interviews and field notes were taken during the interviews to facilitate interpretation. The interviews were then transcribed verbatim for analysis. Analysis then proceeded with a careful reading of all field notes and transcriptions several times to gain an overall impression of each individual's comments. From each transcript, words or phrases that reflect the main topics or meaning of each section were identified and annotated using codes. The terms for the code had not been chosen beforehand but emerged as different topics were raised in the notes. The codes were initially broad in meaning to identify the overall topic of the participants' comments. The procedures for organization and analysis of the data involved listing individual meanings or "meaning units" which are the clustered into common categories or "themes" in order to develop textural descriptions of the lived experiences of the participants (Moustakas, 1994). Particular areas of interest included the participants' descriptions of how social relationships have changed over time, the reasons for those changes, whether communication impairments or other health or disease or life events played a role in those changes. The analysis also focused on the role of how and whether adaptation (either positive or negative) may have occurred either at the level of the individual, his or her communication partners, or the level of the broader social 
network. This included experiences related to negative adaptation, such as social isolation and withdrawal on the part of the individual, social interactions as a source of stress, or feelings of abandonment and social exclusion. It also included positive adaptation, such as finding ways of maintaining the network, replacing network losses, dealing with communication-related impairments, or re-evaluating and finding new value in current relationships (e.g. an increased sense of bond or closeness with intimate friends or family). Insights into the ability of older adults with communication impairments to manage their social networks and support needs were investigated, with particular insight from the quantitative findings. Once descriptions and themes had been identified and organized, the transcripts were reviewed again to ensure that no additional topics or meanings emerged. In addition, if any comments or interpretations were not satisfactorily incorporated, study participants were contacted again for additional clarification. If any relevant new data emerged, they were then incorporated in the final description.

Techniques of verification and validation followed those recommended in the literature (Meadows \& Morse, 2000). Verification was accomplished through literature searches, adhering to the phenomenological method, keeping field notes, using an adequate sample, and review of all codes and coded text by at least one committee member.

Integration of Findings from Phase 1 and 2. According to Morgan (2013), there are three potential uses of a qualitative extension to a primarily quantitative project, namely: exploration, investigation, and illustration. Exploration is often used to try and explain an unexpected result in the quantitative data. Investigation might be used to further explore differences between subgroups. Alternatively, illustration is a means of 
providing descriptive information that provides additional detail and insight into the quantitative results. Two of these uses were applicable to the current study. Qualitative methods were used for exploration in order to try to understand unexpected finding from the quantitative phase of the study, namely the role and centrality of social self-efficacy, as discussed in the next chapter. Consequently the individuals who were chosen to participate in the qualitative phase were selected to provide additional insights into this finding. Secondly, the qualitative data were also used in an illustrative mode to provide additional detail and validity to the quantitative results. As the study measures had not previously been used in as diverse a study sample previously, the interview data were valuable in ensuring that the survey measures were valid and provided additional insights, based on comments and experiences of study participants. 


\section{Results}

\section{Analysis Overview}

Results from both study phases and from several types of analyses are reported. With regard to the quantitative study (Phase 1), the descriptive data for the study sample are summarized first. Second, the internal reliability of the study measures is reported. Third, results are reported from a series of multiple regressions used to examine the relationship between communication impairment, social characteristics, and indices of psychological well-being. Fourth, another series of multiple regressions was used to explore the role of health, disability, and social characteristics as moderators or mediators between communication impairment and psychological well-being. Fifth, analysis of some of the open-ended responses from the study sample was used to explore some of these findings and generate questions for further investigation. For the qualitative study (Phase 2), analysis of open-ended interviews is described and the themes that emerged from this phase are summarized. The key findings from Phase 1 and Phase 2 are then synthesized.

\section{Descriptive Characteristics of Phase 1 Participants}

Demographic and health characteristics. The demographic and health data for

all 240 Phase 1 study participants are summarized in Tables 7 and 8 . The average age of the study participants was 73 years and ranged from 65 to 94 . Exactly half of the sample was female (50\%). The majority of participants were retired $(79 \%)$, had received a college education (59\%), and were married or had a long-term partner (69\%). The modal category for household income was between $\$ 25,000$ and $\$ 50,000$ annually (26\%). Most of the participants lived in a house (77\%) and lived with their spouse or partner $(71 \%)$. 
The sample was predominantly White (96\%) and non-Hispanic (99\%).

The average self-rated health of the study participants was 2.55 on a scale of 0 to $4(0=$ Poor, $4=$ Excellent $)$, with the modal category being "very good" $(44 \%)$. On average, participants reported that they had been diagnosed with 2.55 health conditions out of a list of ten common health problems, the most common being high blood pressure/hypertension (52\%), arthritis/rheumatism (45\%), and some type of cancer (41\%). A complete list of diagnoses is included for reference (Appendix K, Table K1). In addition to these conditions, study participants were asked to list other medical conditions that they had been diagnosed with. Those most commonly reported were hearing impairment (35.6\%), head and neck cancer $(28.7 \%)$, prostate problems $(22.1 \%)$, and Parkinson's disease (11.4\%). A list of medical conditions reported by $1 \%$ or more of the sample is also included for reference (Appendix K, Table K2).

With regard to a list of 14 daily activities, on average study participants reported having some kind of difficulty with 3.22 of those activities. The average level of difficulty across all activities was 0.38 on a scale from 0 to $3(0=$ Not difficult at all, $3=$ Very difficult). Participants most commonly reported difficulty climbing stairs (51\%), the ability to bend, kneel, or stoop (51\%), and doing work around the house/yard (39\%).

More information is included for reference (Appendix K, Table K3).

Table 7

Age and Health Characteristics of All Participants

\begin{tabular}{lccl}
\hline Variable name (metric or range of possible scores) & $M$ & $S D$ & Range \\
\hline Age (yrs.) & 73.00 & 6.25 & $65-94$ \\
Number of health conditions (0-12) & 2.55 & 1.72 & $0-9$ \\
Functional limitations-Total number (0-14) & 3.22 & 3.62 & $0-14$ \\
Functional limitations-Mean difficulty (0-3) & 0.38 & 0.52 & $0-2.57$ \\
\hline
\end{tabular}


Table 8

Other Health and Demographic Characteristics of Participants

\begin{tabular}{lr}
\hline Characteristic & Percentage \\
\hline Female & 50.0 \\
Partnership status & \\
Married & 64.1 \\
Widowed & 10.7 \\
Separated & .4 \\
Long-term partner & 5.1 \\
Never married & 3.4 \\
Employment status & \\
Retired & 79.3 \\
Working full-time & 7.7 \\
Working part-time & 7.2 \\
Unemployed & 1.4 \\
Homemaker & .9 \\
Disabled & 3.6
\end{tabular}

Education

High school or less $\quad 10.7$

Associate/trade or vocational/some college $\quad 30.3$

4 year college degree or more $\quad 59.0$

Annual household income

Less than $\$ 25,000$

Between $\$ 25,000-\$ 50,000 \quad 25.9$

Between \$50,000-\$75,000 14.2

Between $\$ 75,000-\$ 100,000 \quad 13.4$

More than $\$ 100,000 \quad 16.8$

Coresidence

$\begin{array}{ll}\text { Live with a spouse/partner } & 70.7\end{array}$

Live with other relatives/friends/room-mates $\quad 7.0$

Live with paid help (e.g. caregiver) $\quad .4$

Live alone $\quad 21.8$

Residence type

House $\quad 76.6$

Apartment / condo / duplex $\quad 17.4$

Trailer $\quad 1.3$

Assisted living facility $\quad 2.1$

Other 2.6


Table 8 , continued

Other Health and Demographic Characteristics of Participants

\begin{tabular}{|c|c|}
\hline Characteristic & Percentage \\
\hline \multicolumn{2}{|l|}{ Race } \\
\hline White & 95.5 \\
\hline Black / African-American & .9 \\
\hline Asian & .5 \\
\hline Mixed race / Other & 1.8 \\
\hline \multicolumn{2}{|l|}{ Ethnicity } \\
\hline Non-Hispanic & 99.1 \\
\hline Hispanic/Latino & 0.9 \\
\hline \multicolumn{2}{|l|}{ Self-rated health } \\
\hline Excellent & 12.6 \\
\hline Very good & 43.9 \\
\hline Good & 30.5 \\
\hline Fair & 12.1 \\
\hline Poor & .8 \\
\hline
\end{tabular}

\section{Reliability Analyses}

Reliability analyses were performed for each of the study measures. The first step was to examine normality. Table 9 shows the mean, skewness, and kurtosis for each scale. Skewness measures the degree of asymmetry of a distribution and is important as a possible indicator of a departure from the normality assumption of multiple regression. Skewness values of less than or equal to 2 were considered acceptable, and all of the scales met this criteria. Kurtosis values were used to assess departures from normal distributions in terms of the peak and tail weight of a given distribution. Kurtosis values of 7 or less were considered acceptable and all of the study scales met this criterion (Curran, West, \& Finch, 1996). Internal reliability analysis was conducted using Cronbach's alpha. Cronbach's alpha is a measure of internal consistency, which gauges 
the degree to which a set of items are interrelated. A high alpha coefficient alpha value is supportive evidence that the scale in question represents a single underlying construct. Generally, values of Cronbach's alpha that are considered acceptable for research purposes range from .70 to .90 (DeVellis, 2003; Nunnally \& Bernstein, 1994). Values of Cronbach's alpha ranged from .66 to .95 across the study measures (Table 9). Reliability estimates for two scales, Social Participation, the Social Self-Efficacy scale, and the Attachment subscale of the Social Support measure, fell slightly below the optimal value of .70.

Association among study measures. The majority of the study measures were significantly correlated with each other, although the magnitude of most correlations was small to moderate (Table 10). Communication scores were significantly correlated with all of the other scales with the exception of the negative interactions scale.

Comparison of communication scores by participant group. The primary communication measure, the Communication Effectiveness Index-Modified (CETI-M) is a previously validated measure for with adults with communication disorders of various kinds. To date, however, this instrument has not been used in as heterogeneous a population as in the current study. Moreover, the wording of the instrument was altered slightly in order to accommodate individuals with hearing impairments. For all of these reasons, additional analyses of reliability and validity were performed for this measure.

In Table 11, the mean scores and ranges for the communication effectiveness measure are summarized by participant group. For all six participant groups, internal reliability was excellent (0.90-0.96). A one-way between-subjects analysis of variance (ANOVA) was conducted to determine if there were differences in communication 
effectiveness score by group. The ANOVA revealed a significant difference between groups, $F(5,230)=4.86, p<.001, \eta^{2}=.096$. Post-hoc comparisons were conducted using Tukey's Honest Significant Differences (HSD) which has been shown to be an acceptable test for this type of comparison (Seaman, Levin, \& Serlin, 1991). Individuals in the "General Otolaryngology" group had significantly higher communication scores than those with neurologic conditions, hearing impairment, and also those in the general category of volunteers (Table 11). There was no significant difference in communication scores between any of the other groups. This finding was consistent with predictions about the relative level of communication-related impairment between the groups. Individuals in the General Otolaryngology group had been screened during chart review for any previous history of speech or hearing evaluations in order to ensure a relatively low incidence of communication difficulties and would be expected to have generally higher scores on the CETI-M.

Scores on the CETI-M were also compared to the other communication items on the survey, including those from the Washington Group Extended Set of Questions on Functioning (survey questions 6-8) and the self-reported item on telephone use from the functional limitation scale. Evidence of criterion validity of the communication measure was provided by the fact that communication scores were significantly associated with each of these items, as predicted. Communication scores were significantly worse in those who used a hearing aid $(M=48.38, S D=13.55)$ than those who did not $(M=$ $54.19, S D=14.82), t(231)=2.78, p=.006$. Poorer communication scores on the CETI$\mathrm{M}$ were also associated with increased difficulty hearing $(r=-.26, p<.001)$, increased 
difficulty communicating with others $(r=-.57, p<.001)$, and increased difficulty using the telephone $(r=-.62, p<.001)$.

\section{Association between communication impairment and functional limitations.}

One of the purposes of the study recruitment method was to recruit a study sample that was more varied in the range of communication skills and functional limitations than is typical in studies of community-dwelling older adults. Figure 9 depicts the range of scores on the CETI-M. As can be seen, the distribution is negatively skewed and scores ranged from the lowest possible score (10) to the maximum (70). In previous studies of community-dwelling older adults, there has been a paucity of individuals at the lower end of the communication measure. In the current study $11 \%$ of the participants had communication in the most severe third of the range of possible scores. This compares to just $1 \%$ of the individuals in the preliminary study conducted individuals from the Later Life Study of Social Exchanges (Palmer et al., 2012). Consequently it appears the study's recruitment strategy was successful in obtaining a sample of individuals with a greater diversity of communication abilities.

The association between communication effectiveness scores on the CETI-M and the number of functional limitations experienced by each individual is depicted in Figure 10. There were many individuals with low levels of communication difficulty who had few functional limitations but there are also individuals present in all four quadrants of the figure. There was a significant correlation between communication effectiveness scores and the number of functional limitations but the correlation was weak, $r=-.34, p<$ .001 . These data suggest that the study's recruitment strategy was successful in obtaining a sample of individuals with communication impairments with both high and low levels 
of functional impairment. In addition, individuals without communication impairments with high and low levels of functional impairments were also represented.

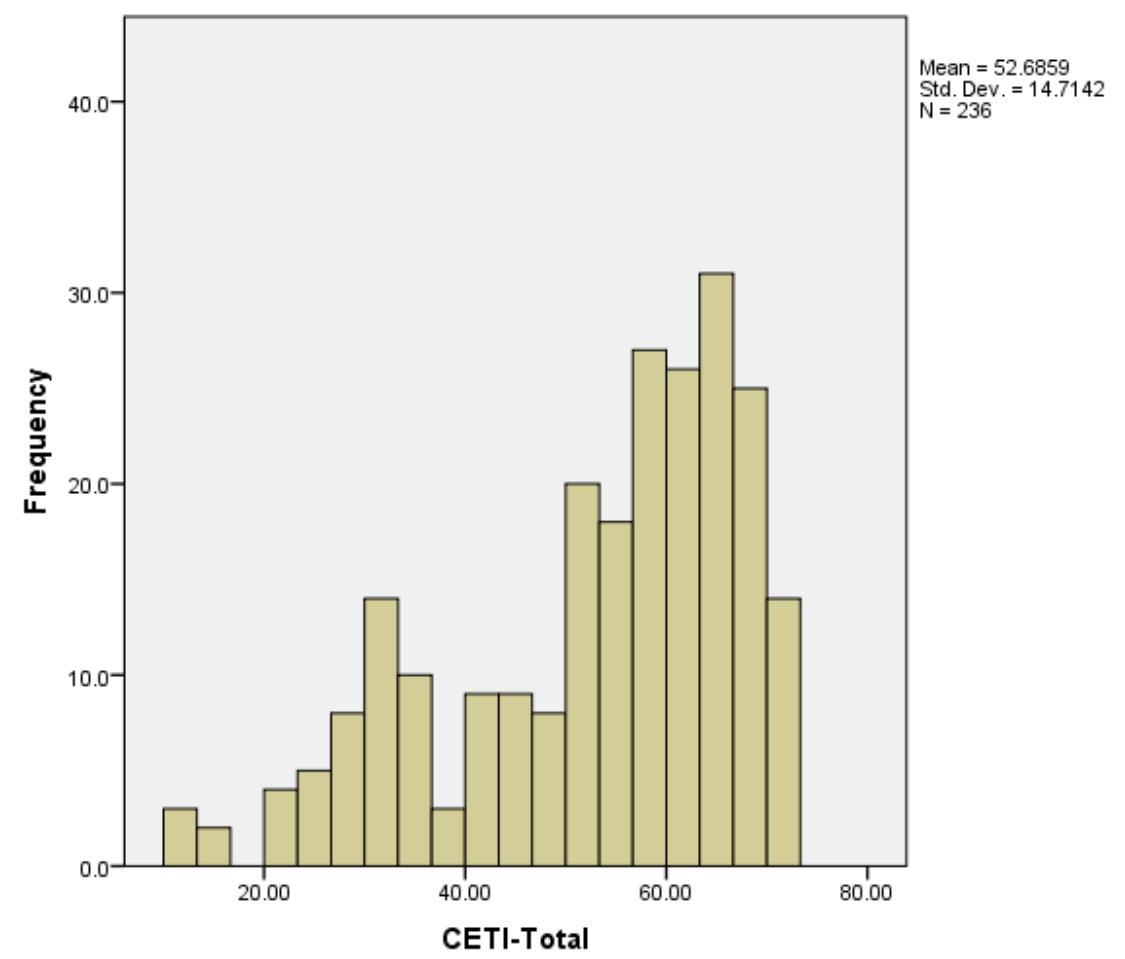

Figure 9. Histogram of communication effectiveness scores on the CETI-M.

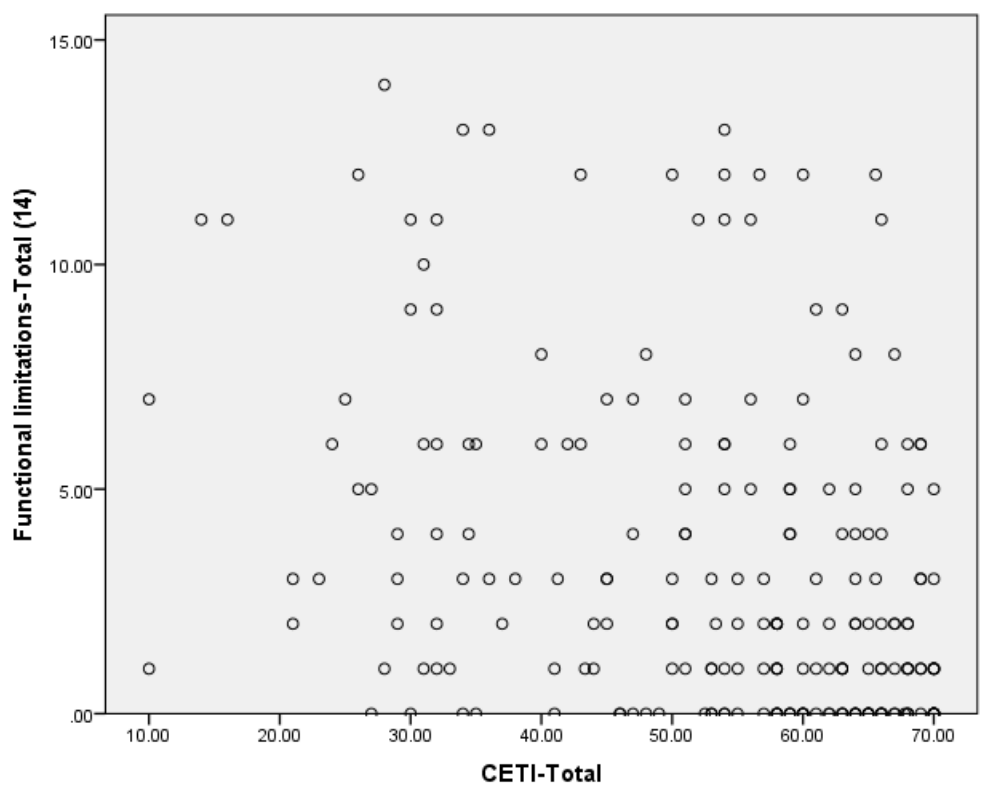

Figure 10. Association between communication effectiveness scores and the number of functional limitations. 
Prevalence of social isolation. Lubben (1988) reported a clinical cut-point for social isolation on the original Lubben Social Network Scale (LSNS) of 20 out of a maximum score of 50 points. More recently a clinical cut-point was been established for a 6-item adaptation of the scale, the LSNS-6, in an international study of older adults atrisk for social isolation (Lubben et al., 2006). For this study, the score was 12 out of 30. To date no cut-point has been established for the LSNS-R but, since the cut-point for the other two versions of the scale was $40 \%$ of the maximum possible score (i.e., a score of 24 out of 60$)$ was selected. By this criterion 29 of the 235 individuals $(12 \%)$ in the sample who had completed this measure were socially isolated. Comparisons were made using chi-square for binary variables and $t$-tests for continuous variables to determine which characteristics differed significantly for those in the most socially isolated category. Socially isolated individuals were significantly less likely to have a spouse or partner, $\chi^{2}(1, N=229)=9.07, p=.003$. No significant differences for age, gender, education, income, number of health conditions, or functional limitations were found. Socially isolated individuals tended to have poorer self-rated health $(M=2.28, S D=$ $1.00)$ than those who were not socially isolated $(M=2.59, S D=.88)$ which approached but did not reach significance, $t(232)=1.74, p=.08$. Individuals who were socially isolated also had lower communication scores on the CETI-M $(M=4.77, S D=1.53)$ than those who were not socially isolated $(M=5.33, S D=1.45)$ which was of borderline significance, $t(229)=1.91, p=.06$. 


\section{Regression Analyses: Predicting Social and Psychological Characteristics}

A series of multiple regressions was performed to examine the relationship between communication impairment, social characteristics, and indices of psychological well-being (Question 1). A total of sixteen multiple regression models were tested. In each model, communication was entered as the primary predictor in addition to the following eight covariates: age, gender, partnership status, education, annual household income, self-rated health, number of health conditions, and functional limitations. This same set of predictors was used for models with each of the following as outcomes of interest: total social network size as well as the family and friends subscales of the social network measures; total social support and the six subscales of the social support measure; frequency of social participation and the number of social activities engaged in; the frequency of negative interactions; social self-efficacy; loneliness; and, depression.

Regression diagnostics. In order to test the homoscedasticity assumption, residual plots were created with the standardized observed residual plotted against the standardized predicted error in order. Inspection of these plots did not suggest any serious violation of this assumption for any of the analyses. The variance inflation factor statistic was used to identify problems with multicollinearity. Values of 6-10 indicate potential concerns (Cohen, Cohen, West, \& Aiken, 2003), but no issues with multicollinearity were found. Several diagnostic indices were inspected to identify possible outliers and influential cases. Outliers on Y were identified by standardized residual scores of over 


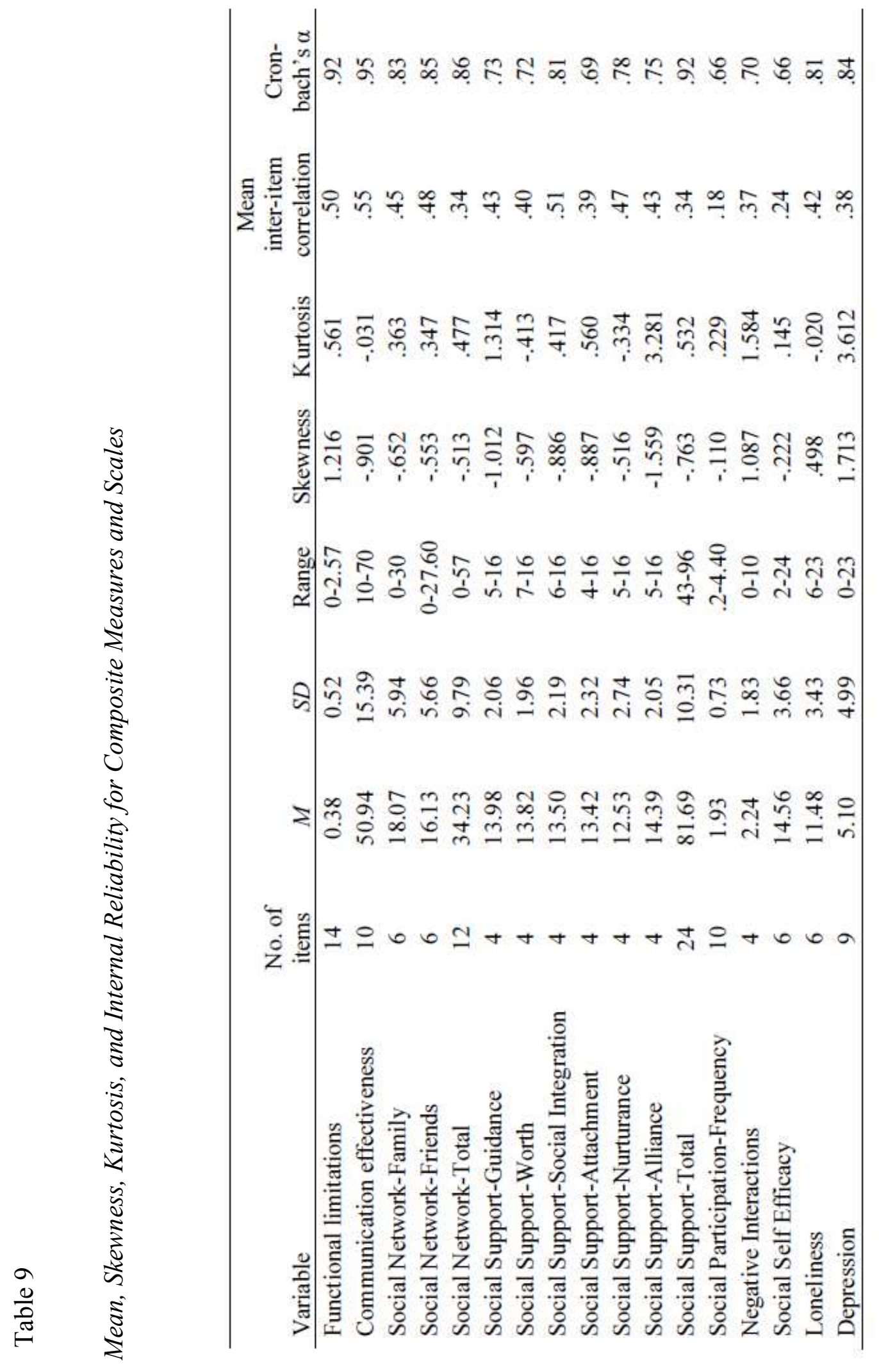


Table 10

Correlation among Study Measures

\begin{tabular}{lccccccc}
\hline Variable & 2. & 3. & 4. & 5. & 6. & 7. & 8. \\
\hline 1. Communication effect. & $.285^{* *}$ & $.338^{* *}$ & .029 & $.365^{* *}$ & $.366^{* *}$ & $-.389^{* *}$ & $-.258^{* *}$ \\
2. Social Network-Total & & $.701^{* *}$ & $-.138^{*}$ & $.543^{* *}$ & $.391^{* *}$ & $-.507^{* *}$ & $-.318^{* *}$ \\
3. Social Support & & & $-.290^{* *}$ & $.510^{* *}$ & $.432^{* *}$ & $-.679^{* *}$ & $-.488^{* *}$ \\
4. Negative Interactions & & & & -.014 & $-.169^{*}$ & $.375^{* *}$ & $.344^{* *}$ \\
5. Social Part.-Frequency & & & & & $.360^{* *}$ & $-.388^{* *}$ & $-.282^{* *}$ \\
6. Social Self-Efficacy & & & & & & $-.533^{* *}$ & $-.385^{* *}$ \\
7. Loneliness & & & & & & $.605^{* *}$ \\
8. Depression & & & & & &
\end{tabular}

$* * p<0.01$ level; * $p<.05$.

Table 11

Mean Communication Effectiveness Scores by Subgroup

\begin{tabular}{lccc}
\hline Group & $M$ & $S D$ & Range \\
\hline A. Benign voice disorders & 57.94 & 12.88 & $24-70$ \\
B. Neurologic conditions & 47.00 & 13.18 & $14-70$ \\
C. Head and neck cancer & 51.18 & 15.51 & $20-70$ \\
D. Hearing impairment & 46.49 & 15.66 & $21-70$ \\
E. General Otolaryngology & 60.69 & 9.34 & $31-70$ \\
F. Volunteers & 50.94 & 15.39 & $10-70$ \\
\hline All & 52.69 & 14.71 & $10-70$ \\
\hline
\end{tabular}




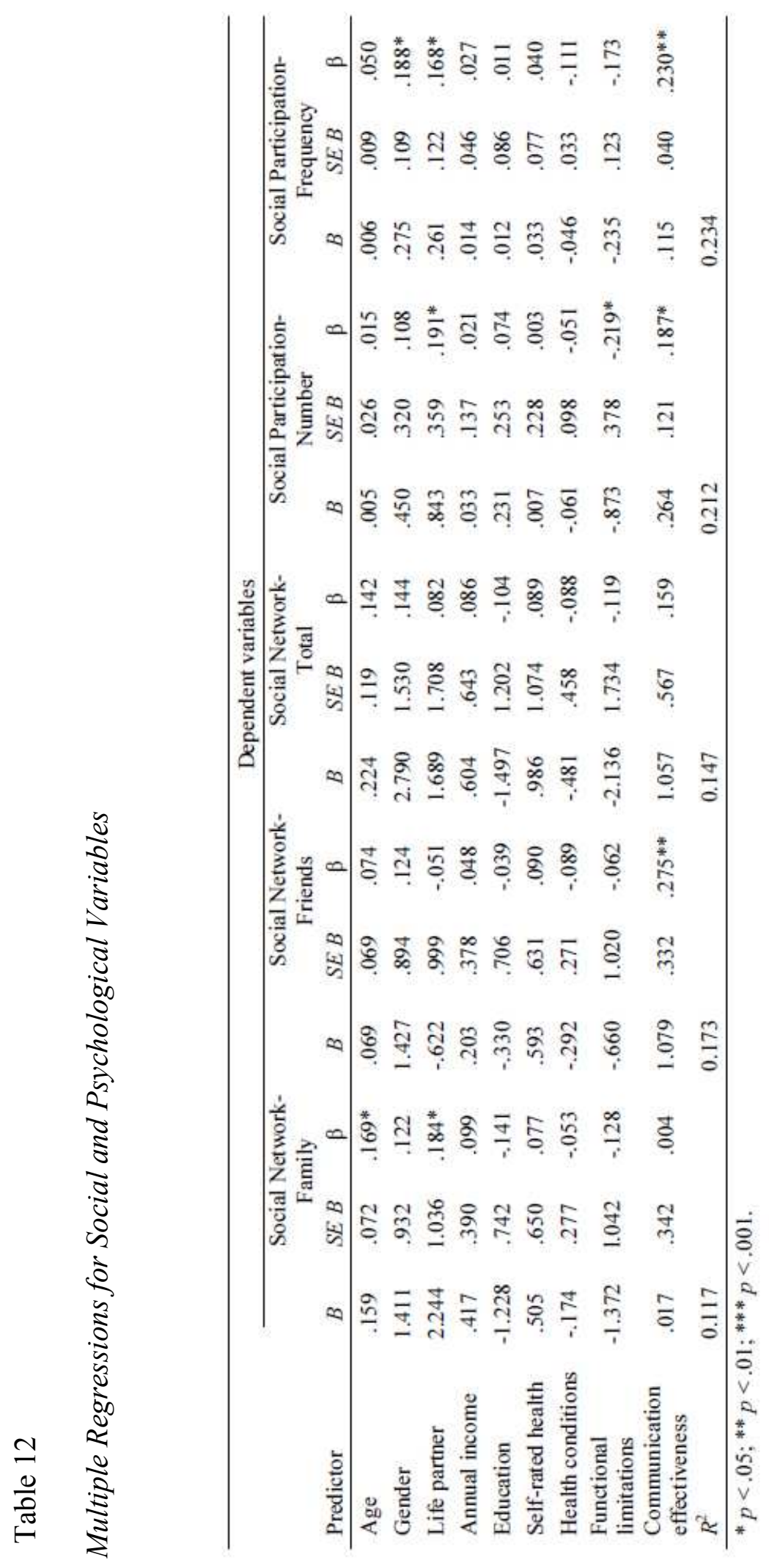




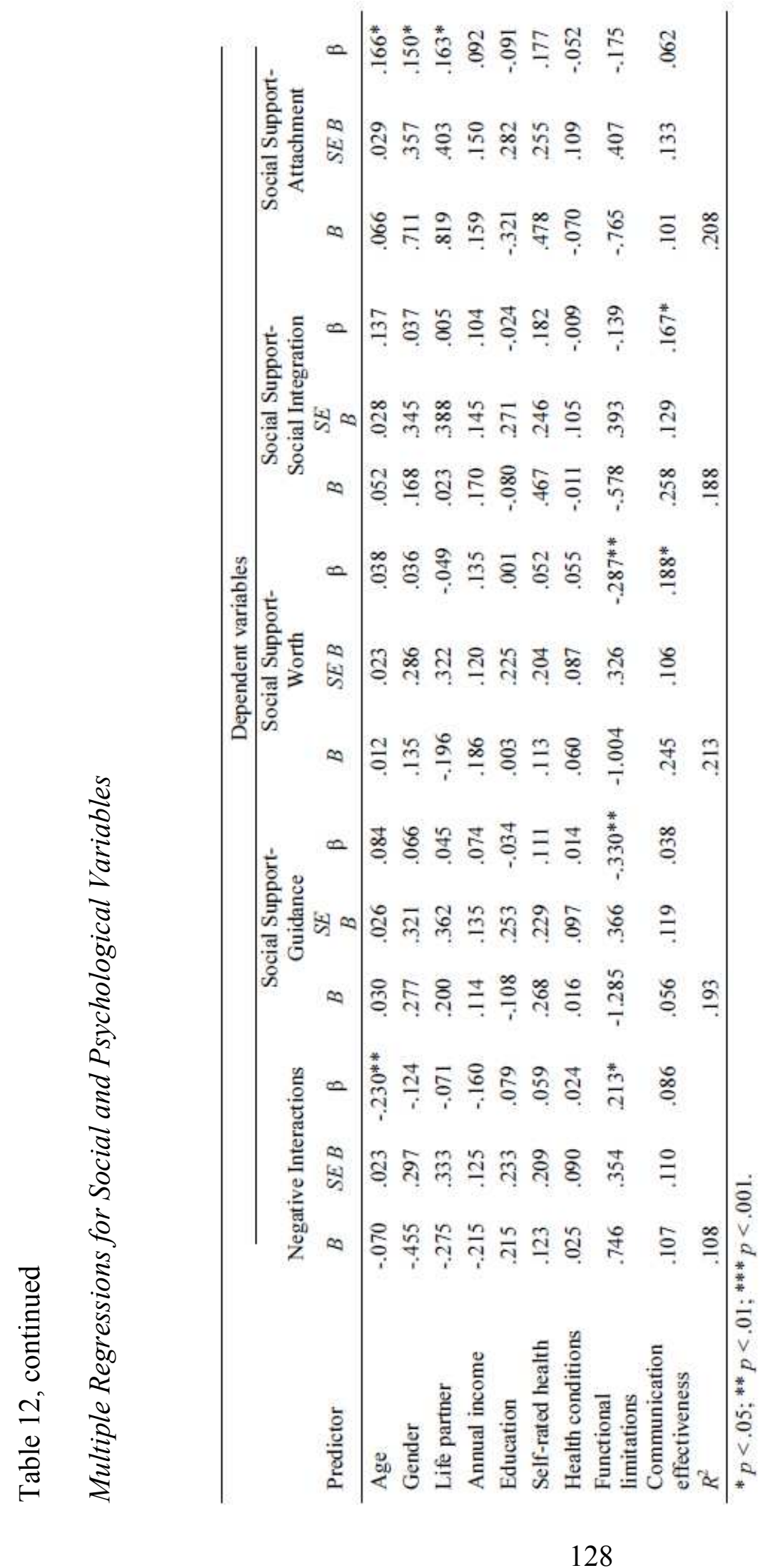




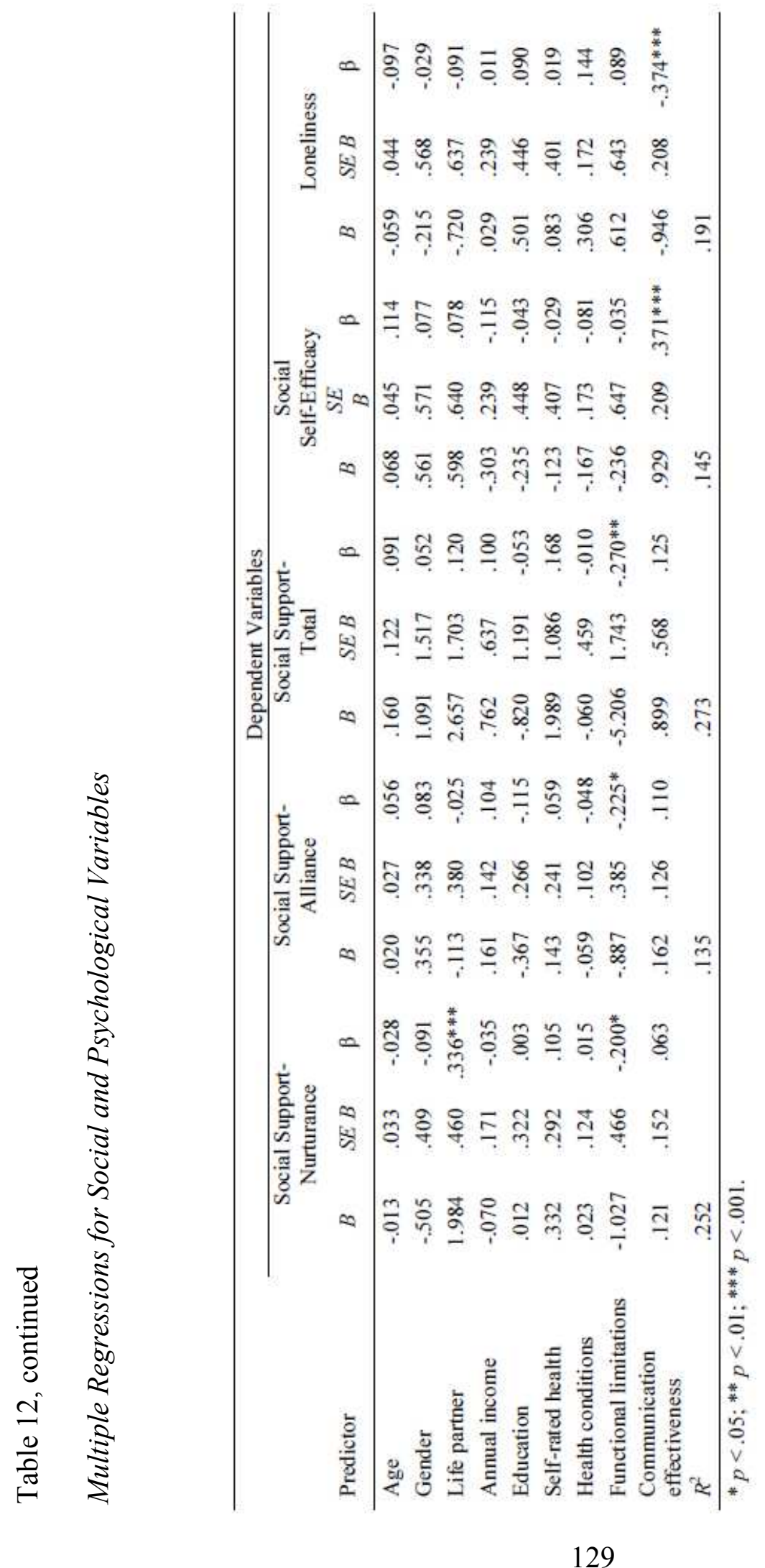




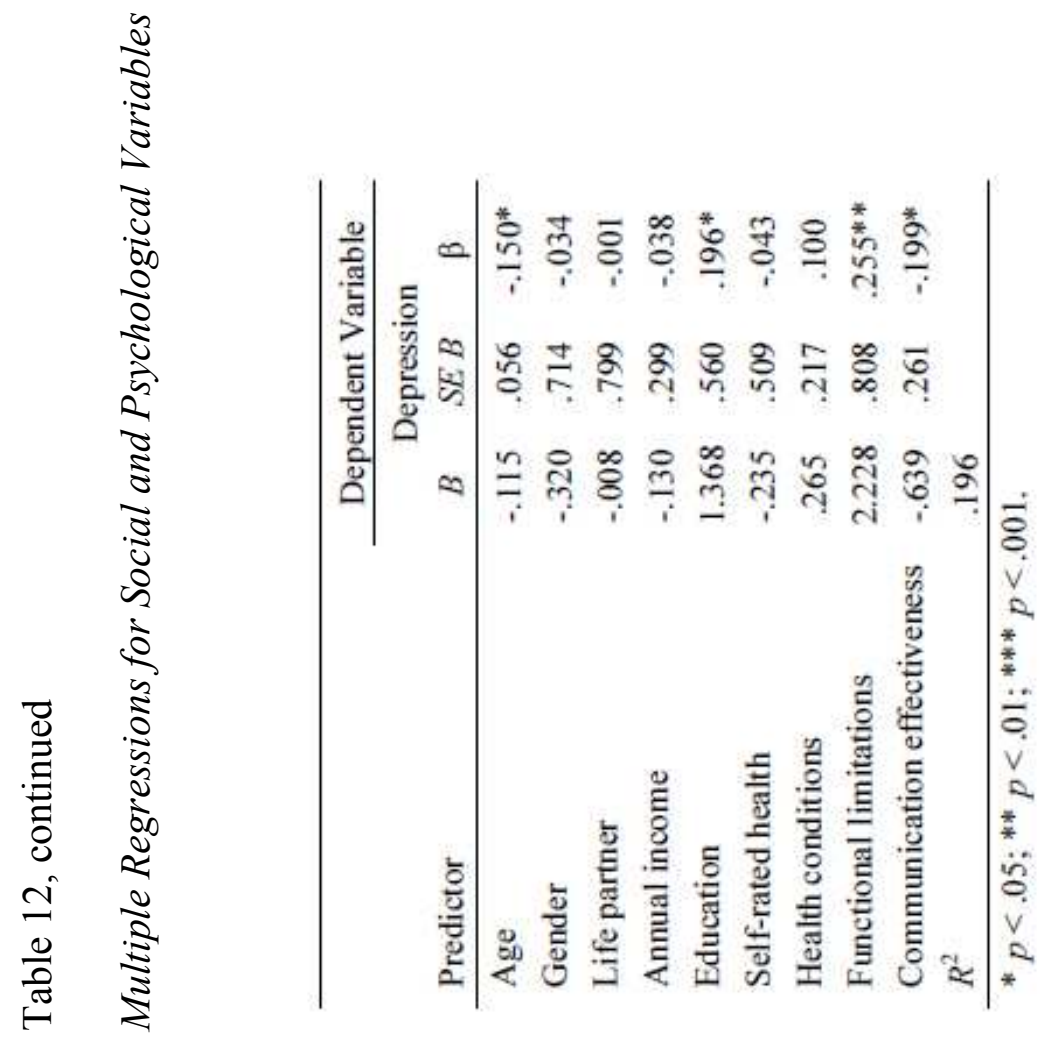


3.0 (Neter, Kutner, \& Nachtsheim, 2004). Outliers on X were identified by leverage values of .2 or greater. Influential cases are cases that may have an undue influence on either the choice of the variables in the model or the accuracy of the regression coefficients and the associated standard errors. Cases were considered problematic if Cook's Distance values were over 1.0. When outliers or influential cases were identified, a series of corrective steps was taken. The values were first examined to determine if a scoring or data entry error was present. If not, the regression was calculated both with the cases included and excluded from the regression model to determine their influence on the results. If no important differences were found, the outlying values were retained in the final model and these results reported. Upon examination, none of the outliers identified had any impact on communication measure in the final model and therefore all cases were retained in the analyses reported.

Regression results for social and psychological characteristics. The results of the sixteen multiple regressions for the social and psychological measures of interest are displayed in Table 12. Three regressions were performed for social network characteristics. In the first model, the family component of the social network was the outcome of interest. Two variables were significant predictors for the family social network, namely age, $b=.159, S E=.072, \beta=.169, p<.05$, and the presence of a life partner, $b=2.244, S E=1.036, \beta=.184, p<.05$. Overall, these variables predicted approximately $12 \%$ of the variance in the family social network, $R^{2}=.117, F(9,167)=$ $2.456, p<.05$. In the second model, the friends component of the social network was the outcome of interest. Communication effectiveness was the only significant predictor for 
the friends social network, $b=1.079, S E=.332, \beta=.275, p<.01$. Overall, this model predicted approximately $17 \%$ of the variance in the friends social network, $R^{2}=.173$, $F(9,165)=3.837, p<.001$. In the third regression, the total social network (i.e., friends and family networks combined) was the outcome of interest. In this model, no variable was a significant predictor of the total social network. This model predicted approximately $15 \%$ of the variance in the total social network, $R^{2}=.147, F(9,166)=$ $3.186, p=.001$.

Two regressions were performed relating to aspects of social participation. In the first, the number of social activities engaged in during the previous month was the outcome of interest. Three variables were significant predictors for the number of social activities, namely the presence of a life partner, $b=.843, S E=.359, \beta=.191, p<.05$, functional limitations, $b=-.873, S E=.378, \beta=-.219, p<.05$, and communication effectiveness, $b=.264, S E=.121, \beta=.187, p<.05$. This model predicted approximately $21 \%$ of the variance in the number of social activities, $R^{2}=.212, F(9,157)=4.705, p<$ .001 . In the second regression, the frequency of social participation during the previous month was the outcome of interest. Three variables were significant predictors for the frequency of social participation, namely female gender, $b=.275, S E=.109, \beta=.188, p$ $<.05$, the presence of a life partner, $b=.261, S E=.122, \beta=.168, p<.05$, and communication effectiveness, $b=.115, S E=.040, \beta=.230, p<.01$. This model predicted approximately $23 \%$ of the variance in the frequency of social participation, $R^{2}=$ $.234, F(9,168)=5.695, p<.001$. 
One regression was performed with the frequency of negative interactions as the outcome of interest. Two variables were significant predictors for the frequency of negative interactions, namely age, $b=-.070, S E=.023, \beta=-.230, p<.01$, and functional limitations, $b=.746, S E=.354, \beta=.213, p<.05$. This model predicted approximately $11 \%$ of the variance in the frequency of negative interactions, $R^{2}=.108, F(9,166)=$ $2.224, p<.05$.

A total of seven regressions were performed relating to aspects of social support. In the first, guidance was the form of social support that was the outcome of interest. Only one variable was a significant predictor for guidance, namely functional limitations, $b=-1.285, S E=.366, \beta=-.330, p<.01$. This model predicted approximately $19 \%$ of the variance in guidance support, $R^{2}=.193, F(9,167)=4.444, p<.001$. In the second regression, reassurance of worth was the outcome of interest. Two variables were significant predictors for reassurance of worth, namely functional limitations, $b=-1.004$, $S E=.326, \beta=-.287, p<.01$, and communication effectiveness, $b=.245, S E=.106, \beta=$ $.188, p<.05$. This model predicted approximately $21 \%$ of the variance in reassurance of worth, $R^{2}=.213, F(9,166)=5.001, p<.001$. In the third regression, social integration was the outcome of interest. Only one variable was a significant predictor for social integration, namely communication effectiveness, $b=.258, S E=.129, \beta=.167, p<.05$. This model predicted approximately $18 \%$ of the variance in social integration, $R^{2}=.188$, $F(9,166)=4.274, p<.001$. In the fourth regression, attachment was the outcome of interest. Three variables were significant predictors for attachment, namely age, $b=.066$, $S E=.029, \beta=.166, p<.05$, female gender, $b=.711, S E=.357, \beta=.150, p<.05$, and 
the presence of a life partner, $b=.819, S E=.403, \beta=.163, p<.05$. This model predicted approximately $21 \%$ of the variance in attachment, $R^{2}=.208, F(9,167)=4.865, p<.001$. In the fifth regression, nurturance was the outcome of interest. Two variables were significant predictors for nurturance, namely the presence of a life partner, $b=1.984, S E$ $=.460, \beta=.336, p<.001$, and functional limitations, $b=-1.027, S E=.466, \beta=-.200, p$ $<.05$. This model predicted approximately $25 \%$ of the variance in nurturance, $R^{2}=.252$, $F(9,166)=6.216, p<.001$. In the sixth regression, reliable alliance was the outcome of interest. Only one variable was a significant predictor for reliable alliance, namely functional limitations, $b=-.887, S E=.385, \beta=-.225, p<.05$. This model predicted approximately $14 \%$ of the variance in nurturance, $R^{2}=.135, F(9,166)=2.880, p<.01$. In the seventh regression, overall social support was the outcome of interest. Only one variable was a significant predictor for total social support, namely functional limitations, $b=-5.206, S E=1.743, \beta=-.270, p<.01$. This model predicted approximately $27 \%$ of the variance in social support, $R^{2}=.273, F(9,165)=6.894, p<.001$.

Three regressions were performed relating to aspects of self-efficacy and psychological function. In the first regression, social self-efficacy was the outcome of interest. Only one variable was a significant predictor for social self-efficacy, namely communication effectiveness, $b=.929, S E=.209, \beta=.371, p<.001$. This model predicted approximately $15 \%$ of the variance in social self-efficacy, $R^{2}=.145, F(9,168)$ $=3.153, p<.01$. In the second regression, loneliness was the outcome of interest. Only one variable was a significant predictor for loneliness, namely communication effectiveness, $b=-.946, S E=.208, \beta=-.374, p<.001$. This model predicted 
approximately $19 \%$ of the variance in loneliness, $R^{2}=.191, F(9,168)=4.410, p<.001$. In the third regression, depression was the outcome of interest. Four variables were significant predictors for depression, namely age, $b=-.115, S E=.056, \beta=-.150, p<.05$, education, $b=1.368, S E=.560, \beta=.196, p<.05$, functional limitations, $b=2.228, S E=$ $.808, \beta=.255, p<.01$, and communication effectiveness, $b=-.639, S E=.261, \beta=-.199$, $p<.05$. This model predicted approximately $20 \%$ of the variance in depression, $R^{2}=$ $.196, F(9,162)=9.900, p<.001$.

In summary, communication effectiveness was an independent predictor for eight of the sixteen outcomes of interest. Communication effectiveness was significantly associated with the friends component of the social network, both aspects of social participation (i.e., the number and frequency of social activities), two aspects of social support (i.e., reassurance of worth and social integration), social self-efficacy, loneliness, and depression.

\section{Physical Disability, Social Support, and Social Self-Efficacy as Moderators}

Previous research has suggested that the relationship between communication impairment and psychological well-being might be moderated by other variables. Some studies have reported moderating role for social support (e.g., Frankel \& Turner, 1983) which would be consistent with stress and coping theories that conceptualize social support as a stress "buffer." Other studies have reported that there might be an interaction between physical and sensory impairments (Kempen et al., 1998). Moderation, also referred to as a statistical interaction or a contextual effect, indicates that a relationship between the independent and dependent variables varies as a function of another variable, 
the moderator. To test whether such a relationship exists, a product variable is created by multiplying the predictor and moderator and the main effects variables and the interaction variable are entered into the regression simultaneously (Cohen, Cohen, West, \& Aiken, 2003). Initially, multiple regressions were performed with loneliness and depression as the outcomes of interest. First, communication effectiveness using the CETI-M and functional limitations were examined as predictors (Question 2). The centered main effects variables and the interaction term were included together in each model. The model also included: age, gender, partnership status, education, household income, selfrated health, and number of health conditions as covariates. Social support as a moderator of the relationship between communication effectiveness and psychological well-being was also investigated (Question 3) using the same covariates. Subsequently, social selfefficacy was examined as a possible moderator between communication effectiveness and either social support or social network size (Question 5).

Physical disability as a moderator of psychological well-being. In the first model, loneliness was the outcome of interest. Communication effectiveness was a significant predictor of loneliness, $b=-.094, S E=.021, \beta=-.373, p<.001$. Functional limitations were not significantly associated with loneliness, however, $b=.601, S E=$ $.691, \beta=.087, p=.386$. The interaction between communication effectiveness and functional limitations was not significant also, $b=-.001, S E=.031, \beta=-.004, p=.963$, which suggests that the effect of communication effectiveness on loneliness was not affected by the severity of co-occurring functional limitations. Overall, these variables 
predicted approximately $19 \%$ of the variance in loneliness, $R^{2}=.191, F(10,167)=3.945$, $p<.001$.

In the second model, depression was examined. Communication effectiveness was a significant predictor of depression, $b=-.063, S E=.027, \beta=-.195, p=.020$. Functional limitations were significantly associated with depression also, $b=2.150, S E=$ $.867, \beta=.246, p=.014$. The interaction between communication effectiveness and functional limitations was not significant, however, $b=-.010, S E=.040, \beta=-.020, p=$ .799. These variables accounted for approximately $15 \%$ of the variance in depression, $R^{2}$ $=.148, F(10,166)=4.063, p<.001$.

Social support as a moderator of psychological well-being. The moderating effect of social support was examined next (Question 3). Communication effectiveness was a significant predictor of loneliness, as before, $b=-.070, S E=.016, \beta=-.272, p<$ .001. Social support also significantly associated with loneliness also, $b=-.252, S E=$ $.022, \beta=-.709, p<.001$. The interaction between communication effectiveness and social support was not significant, however, $b=-.001, S E=.001, \beta=-.046, p=.419$. Overall these variables predicted approximately $55 \%$ of the variance in loneliness, $R^{2}=$ $.551, F(11,162)=18.061, p<.001$.

With regard to depression, communication effectiveness was a significant predictor, $b=-.053, S E=.025, \beta=-.162, p=.032$. Social support was significantly associated with depression also, $b=-.212, S E=.033, \beta=-.470, p=.001$. The interaction between communication effectiveness and functional limitations was not significant, however, $b=-.003, S E=.002, \beta=-.097, p=.151$. Overall these variables predicted 
approximately $37 \%$ of the variance in depression, $R^{2}=.368, F(11,161)=8.528, p<$ .001 .

\section{Social self-efficacy as a moderator of social support and social network size.}

Social self-efficacy was also investigated as a possible moderator of the relationship between communication effectiveness and either social support or social network size (Question 5). Communication effectiveness was not a significant predictor of social support, $b=-.002, S E=.056, \beta=-.003, p=.973$. Social self-efficacy was significantly associated with social support, $b=1.152, \mathrm{SE}=.189, \beta=.396, p<.001$. The interaction between communication effectiveness and social self-efficacy was not significant, however, $b=.006, S E=.014, \beta=.027, p=.668$. Together these variables predicted approximately $41 \%$ of the variance in social support, $R^{2}=.409, F(11,162)=10.192, p<$ .001 .

Communication effectiveness was not a significant predictor of social network size, $b=.049, S E=.056, \beta=.073, p=.386$. Social self-efficacy was significantly associated with social network size, $b=.983, S E=.189, \beta=.368, p<.001$. There was a significant interaction between communication effectiveness and social self-efficacy also, $b=.031, S E=.014, \beta=.160, p=.024$. These results indicate that the significant association between social self-efficacy and social network size may be affected by the co-occurrence of a communication impairment. These variables accounted for approximately $30 \%$ of the variance in social network size in the sample, $R^{2}=.297, F(11$, $163)=6.256, p<.001$. 


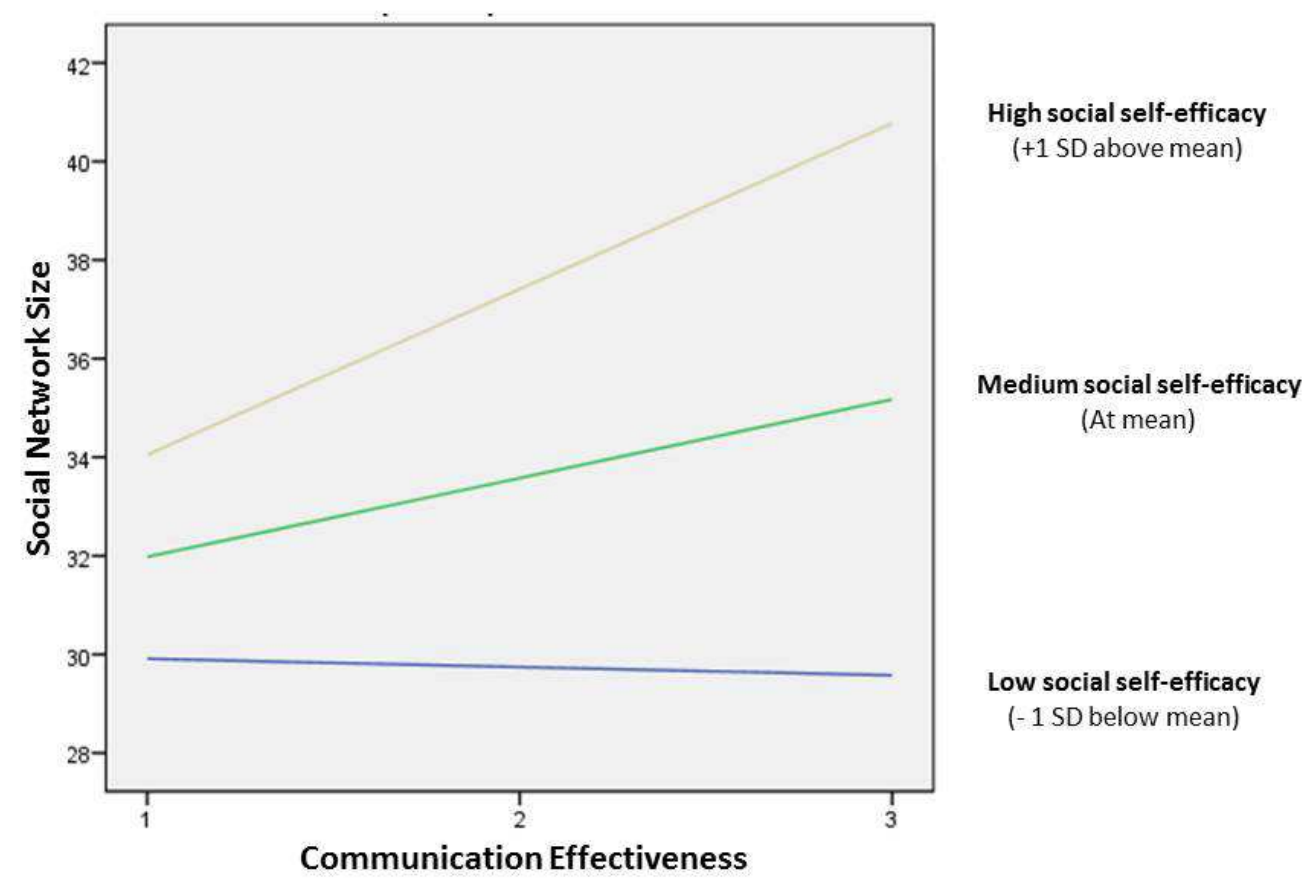

Figure 11. Simple slopes for social network size regressed on communication effectiveness at three values of social self-efficacy.

To further explore the nature of the interaction, a test for simple slopes was then conducted. The results suggested that communication effectiveness was associated with social network size for those with medium and high levels of social self-efficacy. For those with higher levels of social self-efficacy (one standard deviation SD above the mean), communication effectiveness was significantly associated with social network size, $b=2.89, S E=.11, \beta=.43, p<.001$. For those with average social self-efficacy a weaker but significant effect was found, $b=1.51, S E=.07, \beta=.22, p=.001$. For those with low levels of social self-efficacy, however, communication effectiveness was not 
significantly associated with social network size, $b=.13, S E=.08, \beta=.02, p=.81$.

These findings are depicted in Figure 11.

\section{Social Relationship Variables as Mediators of Psychological Well-Being}

Mediation is hypothesized as a causal chain in which one variable affects a second variable which, in turn, affects the outcome of interest. This intervening variable (the mediator) helps to account for the relationship between the independent variable and the dependent variable. When there is no direct effect between the independent and dependent variables after controlling for the mediator, full mediation is supported (Figure 12). When the independent variable has a direct effect on the dependent variable in addition to the indirect effect through the moderator, partial mediation is supported (Figure 13).

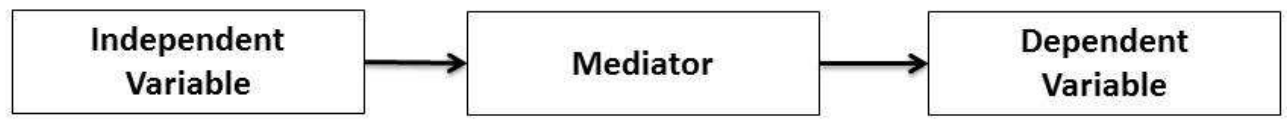

Figure 12. Full mediation.

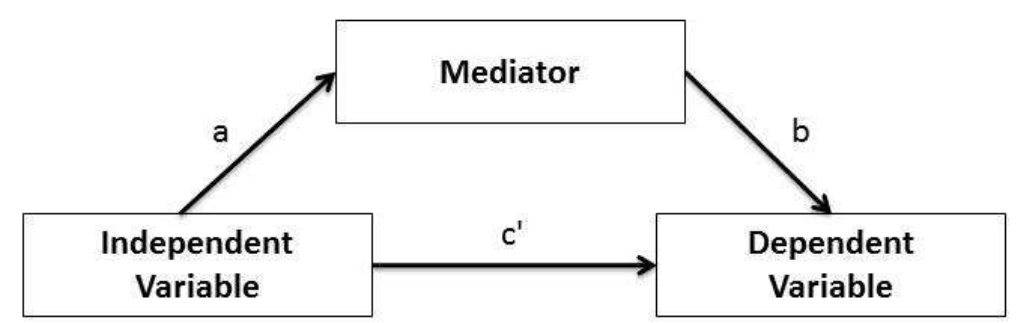

Figure 13. Partial mediation. 
When the hypothesis of mediation by multiple potential mediators is entertained, multiple mediation is an appropriate analytic strategy for analyzing the relative contribution of each mediator (Preacher \& Hayes, 2008). By including several mediators in a single model, researchers can determine the relative magnitudes of the specific indirect effects associated with all of the mediators. In other words, including several mediators in the same model is one way to test a variety of different theories about the relationship between the key variables. Since communication effectiveness had been a significant independent predictor of several parameters of social relationships, a number of models were tested to investigate mediation. Six different social characteristics were investigated as potential mediators of psychological well-being, based on the findings from the multiple regressions (Table 12), namely: the friends subscale of the social network scale; the reassurance of worth and social integration subscales of the social support measure; both measures of social participation (i.e., the frequency of social participation and the number of social activities engaged in); and, social self-efficacy. First, each of these variables was entered as a potential mediator into a model with communication as the independent variable and loneliness as the outcome variable (Figure 11). The model also controlled for the same eight covariates used previously, namely: age, gender, partnership status, education, income, self-rated health, number of health conditions, and functional limitations. Evidence supporting mediation was obtained if both the $\mathrm{a}$ and $\mathrm{b}$ pathways were significant in the model. After testing each of the social variables individually, all of those found to be mediators were then entered into a model in which all of the mediational variables were tested simultaneously. The 
relationships that remained significant in this model were then reported. After this process had been completed with loneliness as the dependent variable the same process was then repeated for depression.

Mediators between communication effectiveness and loneliness. The results supported partial mediation between communication effectiveness and loneliness for all six of the social variables examined. Bootstrapping results indicated a significant indirect effect for all six of these variables, and they were subsequently entered into a model with communication effectiveness and the eight covariates as the independent variables with loneliness as the dependent variable. Results suggested that social self-efficacy partially mediated the relationship between communication effectiveness and loneliness. The indirect pathway for social self-efficacy was the only one that remained statistically significant, $b=-.016, S E=.008,95 \% \mathrm{CI}=-.033,-.003$ (Table 13). Communication effectiveness was significantly associated with loneliness after controlling for the mediators, $b=-.036, S E=.018, p<.05$, consistent with partial mediation. Approximately $57 \%$ of the variance in loneliness was accounted for by the predictors, $R^{2}=.571, F(15$, $147)=13.032, p<.001$. The indirect effect was tested using a bootstrap estimation approach with 1,000 samples (Shrout \& Bolger, 2002). These results indicated that the indirect coefficient was significant, $b=-.042, S E=-.079,95 \% \mathrm{CI}=-.079,-.011$. This model is illustrated in Figure 14.

Mediators between communication effectiveness and depression. The same process was then repeated with depression as the outcome of interest. When each of them was tested individually in a model containing the eight covariates, the results supported 
full mediation between communication effectiveness and depression for three of the six variables examined. The social characteristics that were found to be mediators were as follows: the friends subscale of social network measure; the reassurance of worth subscale of the social support measure; and, social self-efficacy. Bootstrapping results confirmed a significant indirect effect for all three of these variables and they were subsequently entered into a model with communication effectiveness as the independent variable, depression as the dependent variable, and the same eight covariates.

\section{Table 13}

Mediation of the Effect of Communication Effectiveness on Loneliness through Social Characteristics in a Simultaneous Model

\begin{tabular}{lcccc}
\hline Variable & $B$ & $S E$ & 95\% CI Lower & 95\% CI Upper \\
\hline Friends network & -.008 & .007 & -.027 & .001 \\
Reassurance of worth & -.003 & .012 & -.014 & .004 \\
Social integration & -.017 & .007 & -.049 & .002 \\
Freq. of social participation & .002 & .006 & -.011 & .018 \\
Num. of social activities & .001 & .006 & -.011 & .016 \\
Social self-efficacy & -.016 & .008 & -.033 & -.003 \\
TOTAL & -.042 & .017 & -.079 & -.011 \\
\hline
\end{tabular}

Note: 1,000 bootstrap samples.

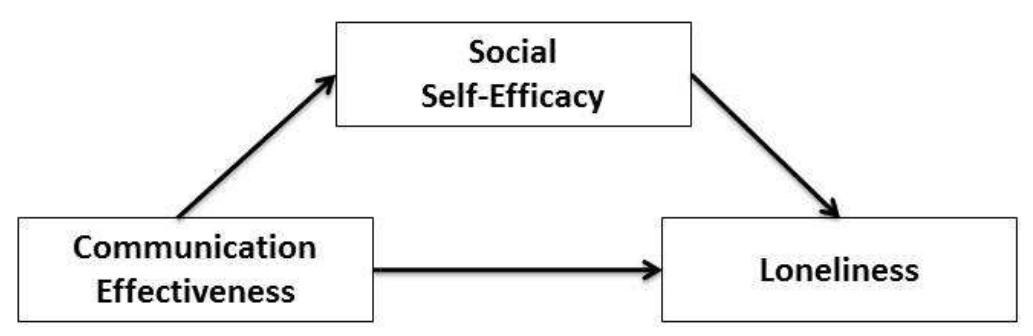

Figure 14. Illustration of the partial mediational model for loneliness. 
Results were consistent with full mediation. Communication effectiveness was significantly associated with reassurance of worth, $b=.026, S E=.011, p<.05$ and reassurance of worth was significantly associated with depression, $b=-.509, S E=.190, p$ $<.01$. Similarly, communication effectiveness was significantly associated with social self-efficacy, $b=.087, S E=.022, p<.001$, and social self-efficacy was significantly associated with depression, $b=-.242, S E=.103, p<.05$. The indirect effect was again tested using a bootstrap estimation approach with 1,000 samples. Bootstrapping results indicated a significant indirect effect for the model as a whole, $b=-.045, S E=.016,95 \%$ $\mathrm{CI}=-.084,-.017$ (Table 14). Reassurance of worth was a significant mediator, $b=-.013$, $S E=.008,95 \% \mathrm{CI}=-.039,-.002$, as was social self-efficacy, $b=-.021, S E=.012,95 \%$ $\mathrm{CI}=-.050,-.002$. Communication effectiveness was no longer a significant predictor of depression when the indirect effect was accounted for, $b=-.011, S E=.026, p=.673$, supporting full mediation. Approximately $34 \%$ of the variance in depression was accounted for by the predictors, $R^{2}=.342, F(12,159)=6.881, p<.001$. This model is illustrated in Figure 15.

Table 14

Mediation of the Effect of Communication Effectiveness on Depression through Social Characteristics in a Simultaneous Model

\begin{tabular}{lcccc}
\hline Variable & $B$ & $S E$ & 95\% CI Lower & 95\% CI Upper \\
\hline Friends network & -.010 & .009 & -.038 & .002 \\
Reassurance of worth & -.013 & .008 & -.039 & -.002 \\
Social self-efficacy & -.021 & .012 & -.050 & -.002 \\
TOTAL & -.045 & .016 & -.084 & -.017 \\
\hline
\end{tabular}

Note: 1,000 bootstrap samples. 


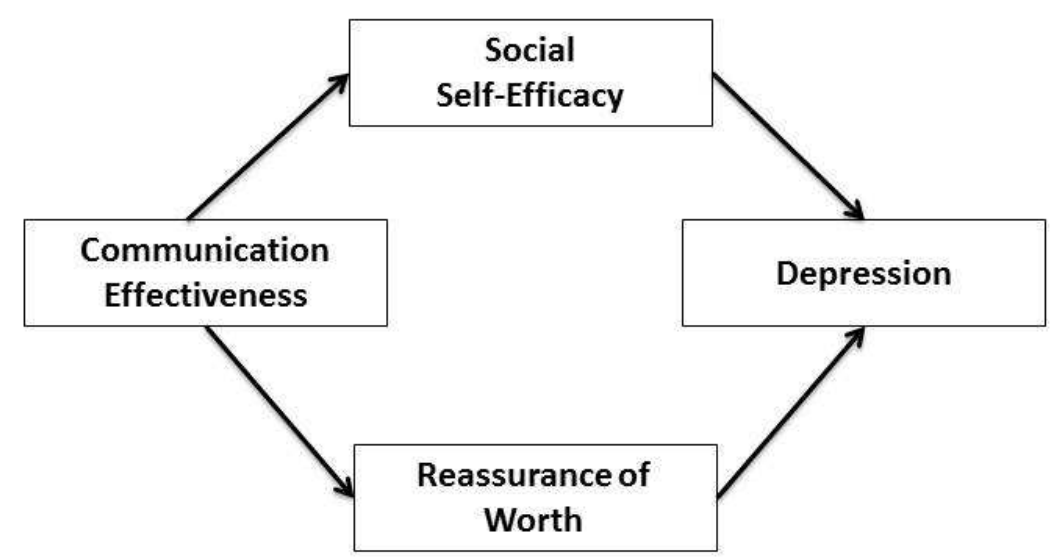

Figure 15. Illustration of the full mediation model for depression.

\section{Perceived Changes in Social Self-Efficacy}

In order to gain additional insight into the concept of social self-efficacy and factors which might affect it, two questions were used in the study survey. After completing the social self-efficacy survey, respondents were asked also whether they felt that these characteristics had changed over time. If they answered "yes" to this question, they were then asked to report what changes had occurred. Of the 236 individuals who completed this item, the majority of respondents $(62 \%)$ reported no changes in their social selfefficacy over time. The responses of the other 90 individuals were then coded as either indicating that they felt that the social self-efficacy was "better" $(n=32)$, "worse" $(n=29)$, or "not reported/unclassifiable" $(n=29)$. Responses fell nearly equally across these three categories. The reasons for these changes were categorized. In those who described an improvement in this area, the reasons given included changes in self-confidence or maturity, improvements in social skills, changed priorities, communication, and health. In some cases individuals gave more than one reason and each one was counted separately. 
Sample comments from each category are listed in Table J2. Of the reasons given for improvement in social self-efficacy, the most common was improved self-confidence or maturity $(18 / 37,49 \%)$. For those who described decreased social self-efficacy, the reasons given included problems with communication, health, changed priorities (including becoming more socially withdrawn), alterations in the social context, and decreased social skills. Sample comments from each of these categories are listed in Table J3. Of the reasons for negative changes in social self-efficacy, the most common reason was changes in communication $(18 / 36,50 \%)$.

\section{Phase 2 Qualitative Investigation Results}

Open-ended interviews were conducted with a selection of study participants for the purposes of exploration and illustration, as described in the Methods section. During the interviews, a variety of topics were addressed, including changes in social relationships across the life-course and the reasons for those changes. The impact of health conditions and communication impairments on relationships with friends, social participation, self-worth, and self-efficacy was explored. Changes in social priorities and social preferences were also discussed. In addition, comments that might elucidate the concept of social self-efficacy and how the findings relating to this measure should be interpreted were explored.

Sampling strategy of Phase 2 participants. A total of fourteen individuals participated in the qualitative interviews. Thirteen participated in face-to-face interviews and one individual completed interview questions via email, due to a combination of factors (including geographical distance and difficulty communicating for long periods 
by phone or Skype). Diversity with regard to etiology of communication impairment and also adequate representation of both genders within the sample was also sought. The average age of the respondents was $72.79(\mathrm{SD}=6.04)$ and ranged from 66 to 83 years of age. Slightly more than half the sample was female (57\%) and most of the sample was currently married (79\%). The respondents had a variety of conditions associated with communication impairments including hearing impairment, stroke, Parkinson's disease, laryngeal cancer, spasmodic dysphonia, vocal fold paralysis, and vocal fold atrophy. The descriptive characteristics of each of the individual participants are listed in Table 15.

Table 15

Descriptive Characteristics of Qualitative Interview Participants

\begin{tabular}{cccll}
\hline ID & Age & Gender & Partnership Status & Communication-related conditions \\
\hline A006 & 80 & F & Married & Vocal fold paralysis \\
A011 & 82 & F & Married & Spasmodic dysphonia, hearing impairment \\
A012 & 80 & M & Married & Vocal fold atrophy, hearing impairment \\
A026 & 83 & F & Widowed & Vocal fold atrophy \\
A030 & 67 & M & Married & Vocal fold atrophy \\
B010 & 71 & F & Long-term partner & Stroke \\
B011 & 67 & M & Married & Parkinson's disease \\
B022 & 74 & F & Married & Parkinson's disease, hearing impairment \\
B030 & 68 & F & Married & Parkinson's disease \\
C025 & 66 & M & Married & Laryngeal cancer \\
C046 & 73 & F & Married & Laryngeal cancer \\
D013 & 71 & M & Married & Hearing impairment \\
D036 & 68 & F & Divorced & Hearing impairment \\
D041 & 69 & M & Married & Hearing impairment \\
\hline
\end{tabular}

Purposive sampling (Cresswell, 2013) was used for the targeted recruitment of individuals to ensure an adequately diverse sample. All participants contacted for participation in the open-ended interviews had indicated a change in their social self- 
efficacy over time on their written questionnaires. Individuals were chosen who indicated

positive changes $(n=5)$ as well as those who indicated negative changes in social self-

efficacy $(n=9)$. Their original responses are summarized in Table 16.

Table 16

Comments by Interview Participants about Changes in Social Characteristics

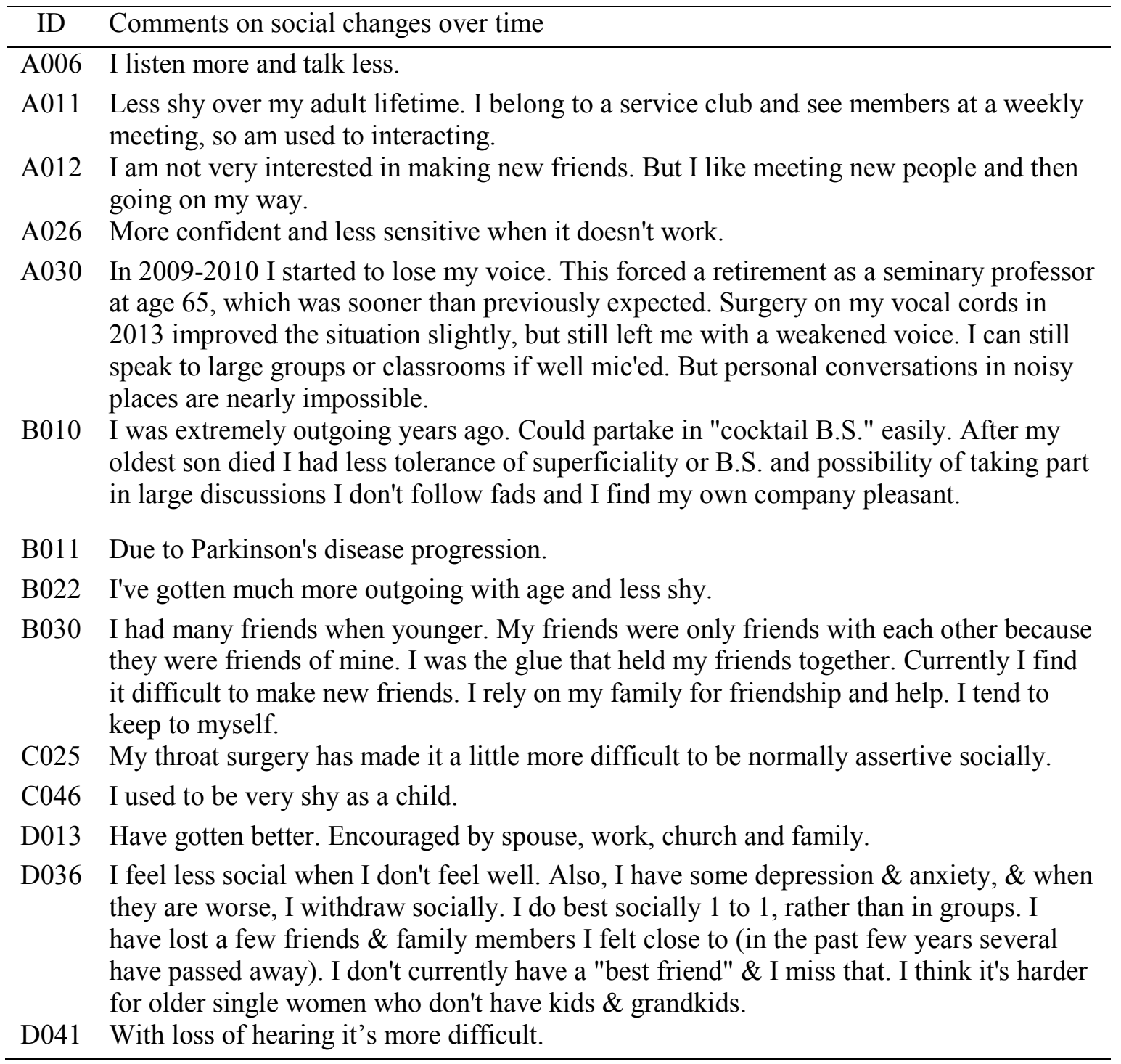


Table 17

Summary of Themes and Subthemes from the Qualitative Interviews

Theme 1: The impact of communication impairments on aspects of daily life.

1a. Feelings and physical symptoms: Annoyance, frustration, discomfort, and fear.

1b. Situations, settings, and activities.

1c. Role losses: Unemployment, forced retirement, and loss of leadership positions.

1d. Relationships with others.

Theme 2: Changes in social orientation and participation.

2a. The importance of relationships: "I never wanted to say I should have" \& "I didn't waste the day!"

2 b. Sociability across the life-course: "If it works, it works."

2c. Relationship losses and continuity: "I'm running out of family."

$2 \mathrm{~d}$. Relocation and the social environment: "Everybody says hello here"

2e. Providing and receiving support: "We know how to treat each other."

Theme 3: Changes in self-perception.

3a. Changes in activity and the impact on identity: "It's a devastating thing to lose who you thought you were."

3b. Perceptions of others: "What do they see that I don't?"

3c. How I sound and seem to me: "It sounds better to him than it does to me"

3d. Finding purpose: "I'm starting to reinvent myself again!"

Themes. Individuals were asked about the impact of their health and health problems on their daily lives currently and also about changes in their social lives over the life-course. Three themes with a number of corresponding subthemes emerged from an interpretation of the data, as summarized in Table 17. The first theme related to the impact of a communication impairment on various aspects of daily life. The second theme related to changes in social orientation and social participation and the reasons for those changes. The third theme related to changes in self-perception and how this had been affected by various factors, including health and disability.

Theme 1: The impact of communication impairments on aspects of daily life. Comments were subdivided into those relating to feelings and physical symptoms, the 
impact on a wide variety of settings, situations, and activities, role losses, as well as the impact on relationships with others.

Subtheme 1a. Feelings and physical symptoms: Annoyance, frustration, discomfort, and fear. A wide variety of emotions were reported by participants. Struggling to be heard or understood was described as being "annoying," "frustrating," and "depressing" by respondents. This was exacerbated in stressful situations when problems became more pronounced, often resulting in greater embarrassment and selfconsciousness. Respondents also emphasized that certain kinds of situations were actively painful or uncomfortable. Two participants who had previously been treated for head and neck cancer reported that talking for any prolonged amount of time produced throat pain, tightness, or coughing. One likened the feeling in her throat after talking for any extended period to "a rose stem with thorns." For those with hearing loss, environmental sounds such as street-noise could be actively painful, making public events and gatherings difficult to negotiate. Comparing the difference between a cochlear implant and a hearing aid in a noisy situation, another participant described it as the difference between sound being "annoying" and "hurting" and continued to avoid loud situations as a result. One participant with hearing loss reported that the annual event Festa Italiana is the "only mob-scene that I go to." She reported that she always went with a friend who had difficulties with crowds also so that, if either one became overwhelmed and needed to leave, it would be understood by the other.

Subtheme 1b. Situations, settings, and activities. Background noise was not only aversive but also posed frequent communication challenges. Many individuals 
commented on their difficulty communicating in noisy situations, such as restaurants, dining-rooms, and even in small group situations such as a book-group. One individual with a hearing impairment reported that background noise severely impaired her ability to understand speech, such as answering the telephone when there was street-noise present. Due to difficulties being understood over the telephone, some individuals minimized phone use with unfamiliar listeners. "I don't like putting people in the position of trying to figure out what I am saying, so face-to-face is better." Long conversations also posed problems. Referring to her voice, one participant with Parkinson's Disease reported: "When I talk for a while it just goes out on me." Despite these difficulties, a number of individuals emphasized that, although problematic, they did not allow their communication difficulties to prevent them participating in a range of activities. "I just ploughed through!" reported one and "I just charge ahead!" reported another.

Many individuals commented with great regret on the loss of the ability to sing or play an instrument. One participant with Parkinson's Disease reported: "I used to sing and I loved to sing but I can't do it. I tried being in a chorus and after the second rehearsal I lost my voice completely." For another, it was no longer possible to participate in congregational singing in his church. And another reported that family musical get-togethers had been one of his favorite activities where he would sing and play harmonica. He can no longer do these thngs because of his hearing loss. With regard to that impact he reported: "I miss it really, really bad. And I probably always will."

Subtheme 1c. Role losses: Unemployment, retirement, and loss of leadership positions. Role losses frequently occurred as a result of health problems, disability, and 
communication impairments. In many ways, these role losses caused some of the greatest disruptions to the lives of the participants. For one individual who had been in charge of a religious training institution in Latin America, a voice impairment caused him to retire and move back to the United States, causing a profound disruption in his life:

In, probably, the last four or five years there my voice was getting weaker and weaker... By mid-2010 I was using a microphone in all my classes but even with that I could not do a 3-hour module. I just couldn't talk that long. So, coinciding with turning 65 we decided that we could no longer do what we were doing down there. And so we left... The cross-cultural shock was huge. And the change to retirement was huge. You go from being in charge of a whole lot of things and a whole lot of people to being in charge of nothing. And besides that when we first arrived, I had these voice issues, these communication issues which were quite depressing.

For another participant treated for head and neck cancer: "Surgery and the subsequent recovery left me looking at the probability of needing to retire... The mill that I worked in was quite noisy so the new voice did not fit there." Although he had originally intended to return to work:

The company said they would save my job but I felt that at least for the time being I could not contribute in the manner I was used to and forty hours a week would be too much. I talked with H.R. The lady there had to give me a box of Kleenex, and not for coughing. So I went down to [Social Security] office. Being full retirement age, the lady there heard my voice and said 'I'll sign you up as disabled.' That was a little shocking.

For another woman, her ability to remain actively involved with her charitable activities was now limited by her voice: “I can't do a fifteen minute talk any more. So that's a major sadness for me... Whatever I do, I'm less likely to be speaking out. To take leadership positions is pretty well impossible." And for one individual with a severe noise-induced hearing loss, his physician mandated him to stop working as a welder with a road-crew: 
That's when the doctor said 'No.' When I lost all of my hearing and the hearing-aids wouldn't even work, the digital hearing aids, he said, 'That's it.' He said 'It's not a matter of if, it's a matter of when you're going to get hit by a car or a truck or something because I can't hear it coming.'... I didn't want to go on Social Security, Disability, take my PERS retirement, I didn't want to do that. He said, 'The system is there for those that need it, you need it, and you're done.'

Retirement was often associated with a loss of identity and, in some cases, had been preceded by a period of marginalization at work, as described by one participant: "When you are approaching retirement age you start becoming invisible. And by the time you retire you're almost totally invisible to the younger generation." For others there was a sense of ambivalence about retirement, as summarized by one participant: "The day I retired from forty years of teaching, I came home and I thought to myself: 'The good news is that I'm not a teacher anymore. And the bad news is that I'm not a teacher anymore.' "

Subtheme 1d: Relationships with others. Communication impairments often interfered with daily interactions with a partner or spouse. Common difficulties included trouble being understood while driving, the need to repeat oneself particularly at a distance or in the context of background noise. In some cases the communication problems of one spouse were exacerbated by those of the other. One participant with Parkinson's Disease noted wryly that:

Somebody said that a qualification for being married to somebody with Parkinson's is being hard of hearing! (Laughs). Because we all have spouses that say, 'Whoa, what did you say?' So I blame it on him and he blames it on me.

As a result she felt that this did have an impact on the nature of their interactions: "I think we get impatient with trying to tell each other something and not being able to." Another 
participant, treated for throat cancer reported that after treatment, "my wife really noticed that our communication level dropped. It's too easy just to wait to be spoken to, so I am trying to be much more conscious of the need to help carry a conversation." Another participant described how his severe hearing loss affected his relationship with his first wife as follows:

I'm sure it was a pain for her, having to repeat herself all the time and write notes. She never learned to Sign with me... But it's hard on somebody, y'know, you have to ask them, 'Huh?' ten times. And they get irritated - 'What do you keep asking me huh for?'

In another instance the apparent lack of sensitivity on the part of a spouse was upsetting:

So I was frequently speaking at a monthly meeting and all and my voice would crack. And my wife would make fun of me and all that kind of thing... Well, it sort of made me feel bad that she kind of, almost laughed at me in a way. And, uh, she didn't mean to be unkind. She's not that way but it hurt me.

For one individual with Parkinson's Disease the biggest change was the fact that his wife was "primarily responsible for communications with family, to some degree and with other friends setting up social dates, planning trips." As a result he described feeling: "Sort of being 'out of the loop'... I feel less in control and less involved." He also noted that he and his wife tended to spend more time with "couples who are friends" and that:

Cocktail parties are the worst. That kind of light banter with strangers is grueling. I wasn't very good at that before, now I'm off-the-charts bad!... But now with the physical limitations and speech and movement, it's a lot more difficult.

With regards to other types of relationships, many respondents emphasized the continuity of their friendships and most important relationships with others even at a distance. Most denied that their health, disability, or long-distance had had a significant 
impact on the relationships that mattered most and reported that many of these relationships were just as close and important as they had always been. Although many relationships were just as emotionally close, communication impairments were often associated with changes in the nature of interactions. In conversation, some reported that there was a tendency on the part of others to interrupt or interject when communication breakdowns occurred which were "kind of rude." Others reported that difficulties in conversations caused them to change their own social behavior. One participant, treated for throat cancer, reported: "I have noticed the diminution of my social communication. It's easy to be quiet and just wait till you know somebody really wants to talk to you." Another participant with Parkinson's Disease reported: "I get tired of people saying, 'What did she say?"' In social situations this changed the nature of the interaction quite significantly. The same participant reported that often:

There are times, like in the Dining Room or whatever, in a group of people, where in the old days (as it were) I would be doing a lot of interjecting... I tend to pick up on things that nobody else thinks are funny and I have to bring up. But I don't even try most of the time. I usually just pull myself out because I will say something like that and maybe the person next to me heard me and might give a little snicker but the table didn't even know I was speaking. It's kind of an invisibility cloak.

Some individuals also reported that a spouse or partner would intervene to facilitate when communication difficulties with others occurred. There were also some reports of negative reactions from strangers. One participant who had been treated for throat cancer sometimes experienced negative reactions from others with regard to the quality of her voice. She found these types of reactions unacceptable: "If they don't like it, they don't have to talk to me. That's the way I feel about it." 
Changes in family dynamics could also occur as a result of communication difficulties. To one respondent, the problems that his wife and daughters experience communication with each other: "Borders on total dysfunction at times." Due to a combination of speech and hearing difficulties, he described a typical family interaction as follows:

It's pretty normal when it's just one-to-one in a quiet environment. But if she's in an environment where there's background noise, like the TV's on, she can't decipher... And she wants to keep up with all her favorite TV shows and her interactions with the kids and stuff but they get frustrated at her and yell at her to get her to try and hear. And that makes her defensive and critical... So that's a very negative impact on communications in the family as a whole.

Theme 2: Changes in social orientation and participation. The second theme related to changes in social orientation and social participation and the reasons for those changes.

Subtheme 2a: The importance of relationships: "I never wanted to say I should have" and "I didn't waste the day!" Many of the interviewees reported that the importance of different types of relationships had changed over the life-course. These changes had occurred for a wide variety of reasons, not always associated with health or disability. For example, the loss of a child was one of the most profoundly significant life events that, for a number of respondents, caused them to re-evaluate their previous priorities. A similarly profound kind of a change also occurred after divorce, for example:

I always felt that I didn't want to say 'I should have.' ... [So] if my children didn't call me, in a month's time I would always call them and make a point to see them... Cause I thought, I don't want to say 'I should... I should have gone there more.' ... I mean, if you want to do something with your kids, do it today because you don't know what tomorrow's going to bring. 
As a result of altered priorities some participants described a loss of interest in former activities. One respondent talked about considering dropping out of a women's riding group but had ambivalent feelings. Asked about the nature of the dilemma, she questioned: “Am I losing part of myself by pulling myself out of groups and things?... [W]hat's going to become of me if I just isolate myself?" A similar loss of interest in group membership was described by another participant who also framed the decision in terms of the desire for more profound connection:

I've lost interest even in my book group that I've been with for 30 years... I feel like I am wasting my time. I'd like to be doing what I really like to do which is hike, read, and study things. And if I meet somebody... [and] I can really talk to them about what interests me, then I am fine.

Some participants commented on their reduced tolerance for superficial relationships and the lack of meaningful contact which had focused their attention on more profound interactions with family members and friends. This type of view was not universal, however. Several individuals described the importance of more casual encounters, companionship, gossip, acquaintances, and new friends. For one participant, who had relocated multiple times over the course of her adult life and always made new friends, the failure to do likewise after relocating to a retirement community was a source of puzzlement and concern:

I have been here going on three years and don't have anyone that I would consider a close friend. It's probably because I don't put into making friends that I probably did when I was young. Or I was just in an atmosphere where there were more people who were likely to be my friends... It just seems strange because... I was in so many different living arrangements as I grew up... I was in lots of situations where I had to start from scratch. But this is one that I started from scratch and didn't get anywhere. 
When asked about why this was important to her, she commented on the importance of companionship and finding commonalities with others in terms of the "personality of other people who kind of look at the world like I look at it.”

Another respondent found herself pondering the meaning of a day that she had initially considered "wasted" because she had not been able to get anything of importance accomplished. After reflecting on all the people she had chatted with and the various conversations that she had taken part in over the course of the afternoon, she came to the realization that, "it was wonderful... I didn't waste the day! It was probably the better part of any of the days."

2b. Sociability across the life-course: "If it works, it works." In terms of sociability over time, responses varied widely. Some respondents emphasized continuity, for example: "I'm a pretty social person and I don't think that's changed." Others described the fact that they had become more outgoing, assertive and less self-conscious over time, such as being less sensitive to criticism: "As I got older, I said 'Hell with it, I don't care what people think. I'll say what I feel as long as it's not rude or immoral.' ’ Another woman reported that, in dealing with people she didn't know, she was "more confident and less sensitive when it doesn't work". She explained this in terms of her reactions to failed interactions, as follows: "I just don't get in a flap! I mean, if it works, it works. If it doesn't, it might later.”

The experience of some individuals, however, caused them to be more reticent in company. After her divorce, one woman reported that she would avoid sharing her opinions with her second husband because of their frequent arguments: 
I just wouldn't say things. I didn't really feel, the last couple of years, like I was really myself saying what I thought I should say... [But] I don't want to have a lot of arguments - so I think you just keep your mouth shut rather than cause that.

For some individuals, their enjoyment of solitary activities was unchanged over time. One man reported that: "I'm a person who enjoys being alone." For others, however, communication impairments were associated reduced social participation. For one woman with hearing loss, the effort and exertion required for periods of social activity needed to be counterbalanced with equivalent amounts of "quiet, alone time." As discussed in the previous section, one woman with Parkinson's Disease described the being treated as though she were wearing an "invisibility cloak" when she was in a group. Another man with Parkinson's Disease commented:

I've always been somewhat introverted. And I suppose that that has gotten worse over time... I enjoy [social situations] but when I participate but I don't carry my own weight... Before, if I made the effort, I could do it. But now it's much more difficult.

One participant treated for throat cancer commented on "the diminution of my social communication. It's easy to be quiet and just wait." Another man with a hearing impairment reported his difficulties in social settings as follows:

[Y]ou can't understand every word that people say. If you lose a word in a conversation, you lose the whole sentence. And I suppose, like most all the rest of hearing-impaired people, you end up kinda being alone in a crowd and pretty soon you do things without anybody. You just end up being alone off by yourself and do things just by yourself. You get tired of saying 'huh?' to everybody all the time... I still do the same things, I just basically do them by myself, pretty much.

2c. Relationship losses and continuity: "I'm running out of family” In the course of the interviews, many individuals described relationship losses for a wide variety of 
reasons. Family members including parents, siblings, and children had passed away. Partners and spouses had been lost to death and divorce. Estrangement from family members had also occurred for a wide variety of reasons. Nonetheless many interviewees also emphasized the long-term continuity of many close relationships over long periods of time, despite health changes and distance. Most people described attempts to maintain these relationships: "I'm running out of family but what family I have left, we're trying to stay together." In some cases the loss of blood relatives was filled by other relatives who were connected by marriage. Many individuals reported no change in their close friendships even though, in some cases, these had to be maintained over long distances. The loss of friends and acquaintances occurred for many reasons. Relocation caused some individuals to lose contact with old friends. Retirement, sometimes in association with health changes, also caused the end of social relationships with friends from work. Following his diagnosis with Parkinson's Disease one man reported, “A few of [my friends] were supportive and asked questions but others kind of fell by the wayside." This occurred about the same time as his retirement:

Well I retired about three or four years ago, so that fact in and of itself meant that I lost contact with a lot of acquaintances, I would call them close friends and acquaintances. So I miss that a lot - that activity, that part of it.

In some cases, the loss of friends over time was replaced by family members: "My two kids are definitely my best friends. Even more so than my husband.” In some cases, disability on the part of family members who required the partner to take on a caregiving relationship, caused a more severe restriction of the social network: 
You generally have to have your world shrink and you start dealing with those things that are vital and suppressing those things that are inconsequential. And pretty soon your walls are pretty much your own home... The health of my family is vital, everything else is inconsequential.

2d. Relocation and the social environment: "Everybody says hello here." For many respondents, the social context of the environment in which they lived afforded varying levels of opportunity for interaction. Those who lived in retirement communities commented favorably on the range of activities and opportunities for social participation that these settings afforded. One woman commented:

Oh, I just say 'hello.' Everybody says 'hello' here... And tell them you don't know what their name is and they say, 'It doesn't matter. Nobody knows what everybody's name is here.'

During interviews, the issue of relocation was frequently mentioned. Many respondents had moved to the Pacific Northwest from elsewhere in the United States, usually in order to be closer to family. The choice of living situation, however, was often based on personal priorities. One participant with Parkinson's Disease described how many of her friends could not understand their decision to move into a retirement community:

Everybody that I knew in the other world thinks that we're absolutely insane to live in this, in an environment like this... 'Why would you want to go to a nursing home?' It's not a nursing home... It's like a big, college dorm where everybody's old! (Laughs).

Not all of the respondents had the means to relocate, however, nor could they all afford to live in this type of setting. One couple who were currently living in mobile home park could not afford the costs associated with moving to a retirement community. Another woman, disabled during adulthood had lived for a number of years in lowincome housing with a number of other older residents "most of whom are frail." More 
recently, however, a new housing policy had resulted in a greater influx of more severely disabled residents. Some of the previous residents had chosen to move out as a result. She had chosen to remain there because of the proximity of the building to stores and transportation made it an "unbeatable location" in her mind. Unfortunately there were fewer residents in the building who could provide her with support.

\section{2e. Providing and receiving support: "We know how to treat each other."}

Participants provided numerous examples of supportive relationships with friends and family members. Many received practical assistance, such as with finances, transportation, household chores, and maintenance. Assistance was also provided in terms of providing accommodation during periods of convalescence and rehabilitation. Emotional support was also frequently available, both in person and from a distance. Support provision was not unidirectional, however, and there were numerous examples of the study participants continuing to provide support and assistance to others. Older adults provided practical, financial, emotional and informational support to those around them, in addition to childcare and other types of services. Interviewees were very aware of the importance of the support that they had received at critical periods in their lives. Family members also provided counseling, support and advice during periods such as after retirement, role-loss, and relocation. The availability of support was not equal for all respondents, however. One woman, who had experienced disabling health problems during adulthood and had divorced without children, commented: "For single older women, it's much tougher." Due to the fact that her siblings were older, in poor health, and geographically distant, her priority had become creating a support network for 
herself. She described her efforts to do so consciously and in very pragmatic terms. Her closest friends were "single, unattached and alone" with no families, because these types of individuals were more likely to provide support to each other and also tended to make their friends a priority. Her "closest friends now all have some kind of chronic illness" which results in greater "sympathy" for someone else in a similar situation. She had created her own support network of "emergency contacts" made up of "younger friends" who could provide assistance to one another, as needed. Being naturally "an introvert," she reported that this made it "even more difficult to put yourself out there." Having worked in a sales environment she noted that: "Making friends is a selling job in a way." She now considered herself to be a "forced extrovert" in that she had "learned how to do it when I have to."

Many participants belonged to support groups of various kinds and commented on the significant role that they had played in their lives. For some individuals, these benefits were purely practical, resulting in better health and physical fitness. For others, however, support groups provided more profound benefits. For one woman with Parkinson's Disease, local support groups had enabled her to form social relationships and develop a network of support almost immediately after relocating to Portland:

In fact, actually having Parkinson's was, kind of my saving grace when we first moved here because those were the first people we met, and they became our first friends... We've kind of become like a family. And I often wonder where I would be and who I would be with if I had just moved here because my kids were here and there was nothing. Y'know, if I never got involved with the whole Parkinson's scene.

The same woman commented about the fact that the support group was the only situation in which she was treated as a peer: 
And the people in the class, y'know, we are very close, very close. In a whole different way... Knowing that you're accepted... [W] know how to treat each other. So you don't have to go through that, y'know, 'This is what I have, but this is what I don't want to happen.' Everybody understands.

Theme 3: Changes in self-perception. The third theme related to changes in selfperception and how this had been affected by various factors, including health and disability.

3a. Changes in activity and the impact on identity: "It's a devastating thing to lose who you thought you were." In some cases, the changes that went along with a health condition or communication impairment were accepted as a normative part of aging and seemed not to be disruptive. One man commented on his hearing loss: "You know I'm an older person, so people expect that!” Another woman summed up the progressive limitations in numerous areas for her husband and herself as follows: "We're getting old!" Even in the case of a progressive neurological disease, the slow progression of changes allowed gradual adjustment as, for example, for one man with Parkinson's Disease: "I take each change as it goes along." For others, however, a sudden change resulted in profound alterations resulting in a crisis, such as with one woman who had suffered a relatively mild stroke:

Well I suffered a great deal because I was making furniture out of leather and stuff and I couldn't do that... So I was feeling my identity had left me because I couldn't do what I used to do.

After the stroke she had some word-finding difficulties and some physical limitations due to a hemiparesis. For her, these physical limitations in particular caused great disruption:

I used to be a tough little kid, I was a tomboy. Sailed sailboats, rode motorcycles and horses all my life. I pushed myself. And that I suffered 
because I could see that I wasn't going to be able to do that. And that was missing. That was part of my so-called identity but I guess it wasn't really. I mean that's who I thought I was.

In terms of understanding the profundity of the changes that someone in this type of situation was going through she advised: "Well, just understand that it's a, it's a devastating thing to lose who you thought you were."

Although a stroke will often cause a sudden change in an individual's abilities, resulting in a period of crisis, it is important to note that this could occur even with gradually progressive conditions. For example, the pastor who had been teaching in South America had noted voice changes over the course of several years. It was not until these changes reached a critical point where he felt that he was no longer able to perform his duties "adequately" that a critical transition was reached. By choosing to accept a forced retirement, he gave up his position, retired, and relocated back to the U.S., resulting in a period of profound transition and personal crisis for him, as described above.

3b. Perceptions of others: "What do they see that I don't?" Many individuals reported that friends and family members had become more "solicitous" about their health and well-being and in some cases maintained more frequent contact as a result of health changes. Due to the inability to drive at night, for example, one man with Parkinson's Disease reported that his adult children came over more often and watched basketball games with him and that, when his wife was out of town, they would "check in" more often. Another woman with Parkinson's Disease reported that her friends would take her arm when they were walking together which she "appreciated" even though she 
felt that: "I don't really need it." In some cases, these types of negotiations caused some levels of disagreement between partners:

$[\mathrm{H}] \mathrm{e}$ is much more careful of me. He always wants to carry things for me or take care of me... [To spouse] I can walk by myself. I don't need you to hold my hand all the time.

Despite being well-intentioned, the "help factor" sometimes caused a degree of ambivalence on the part of the individual that it was directed towards. Some individuals felt that it was over-protective and caused them to be perceived as more disabled than they felt themselves to be:

People that I knew before are very solicitous. Y'know, people that used to - I mean I was just one of everybody else. And now they take my arm when they walk somewhere. I appreciate it. I don't really need it and I don't know how to say, 'Leave me alone.' ... But that is the biggest difference, is the 'help factor'... But it's funny because it does change the relationship. They now, instead of seeing me as just one of them, they see me as 'one of them that needs some help.' But I guess that's who I am! So it's confusing!... I get a lot of... 'Can you do the stairs?' Well, yes I can! I hope I can do stairs for a long time!... Sometimes I wonder, "What are they seeing that I'm not?" But I'm thinking, "I'm the one who's in the body, climbing the stairs." But I must, it must show somewhere... That if there were no outward sign they would forget [about the Parkinson's]... But they, they're all very aware.

Another respondent also wondered how he was perceived by others: "I know when I see other Parkinson's patients, I wonder what my family, how my family looks at me." Questions of how they were perceived by others and the fact that their impairments were obvious to others caused concerns to individuals with other types of communication impairments. One individual treated for throat cancer reported that, "in talking with people that I don't know for the first time, I see myself saying 'Pardon my voice' quite often. I guess that I feel I need to alert them in some way. Everyone seems pretty 
receptive and I have not had what to me were negative experiences. I think I am doing that less now."

3c. How I sound and seem to me: "It sounds better to him than it does to me." In addition to the ways that they were perceived by others, a number of participants described how bothered they were by the changes that they perceived in themselves. For example, one woman with Parkinson's Disease described her realization as follows:

I found a little 'sound test' on the Skype. So I thought, oh, that'll be fun, y'know, if I just... talk to it a little bit and play it back. And I was amazed at the difference from what I thought I was saying to what was coming out in the recording!

She also wondered what would become of her as her voice issues progressed: "I wonder what happens to me when I don't have a significant voice, I mean if it got to that point, because so much of me was my voice." She likened the situation to an athlete who can no longer do their sport anymore. When something that is so central a part of one's identity is removed: "Where do you go from there?"

One of the participants treated for throat cancer described the impact of voice changes as follows: "I feel that I am not as interesting to people any more. Verbal communication used to be a strength." He felt that this had dramatically changed how he was perceived by others: "It's seems like some of the authority and interest of who I was and had to say went away with my normal voice." For another individual with a benign voice disorder, the loss of voice was also associated with a loss of self-confidence and authority:

I felt more self-conscious. There was a time in my life that I was a good speaker... I had what people said was a 'radio announcer's voice.' And I, I 
didn't cultivate that in a way but it was very useful, I think. Useful as a device in terms of meeting people and... it was part of me.

Another woman who had also had to give up public-speaking and leadership positions because of her voice described the difference in the perception of the situation between her spouse and herself:

He can understand me when I think my voice is bad. Right now, to me, my voice is choppy and breaking up. OK and sometimes it's much worse and I have to really push... in order to speak. And he never complains, he never gives me any reason to think it's abnormal. In fact it may sound better to him - it undoubtedly sounds better to him than it does to me!

\section{3d. Finding purpose: "I'm starting to reinvent myself again!" As a result of role}

losses and the changes in physical and communicative ability, many individuals sought purpose through a wide range of activities. Church and voluntary activities, mentoring, and teaching were frequently mentioned, in addition to learning new skills and finding new roles for "keeping up and keeping in touch with people." These activities were associated with increased self-confidence and an opportunity for meaningful contributions to friends, family members, and society at large. Creative activities and classes also provided opportunities for development, self-fulfillment, and redefinition:

There was a period when I was really suffering. Because I thought 'What am I going to do now?' But [my partner] encouraged me in my art... I found some art classes in Portland Community College in abstract art... So then I went out and started going to school and I'm starting to reinvent myself again! (Laughs).

For the pastor who had relocated to the United States after giving up his mission, he described a long, slow process of several years to find new purpose and meaning in his life: 
When I first arrived back, with the voice issues, with the being new to the country again, being new to retirement, I was asking God, 'God what purpose do you have for me? What do you want me to do?' And he didn't show that to me all at once. It was, it came in pieces over the last three years. This was added, then this was added, then this was added, then this was added. Now when I look back over the last three years, I say 'Wow! So that's what you wanted me to do. That's great - I enjoy that!'

\section{Integration of Findings}

The purpose of this study was to gain a better understanding of how communication impairments impact a diverse range of social relationship factors and the psychological well-being of community-dwelling older adults. Each of the significant findings is listed in turn and these findings are then discussed in the next chapter.

\section{Communication impairments were associated with having fewer friends in} the social network and a reduced sense of social integration. After controlling for demographic, health, and disability characteristics, communication was not a significant predictor of overall social network size, nor of the size of the family network. Communication was a significant independent predictor of the friends subscale of the social network measure, however. Those with greater communication difficulty had fewer friends in their social network. In addition, communication impairment was a significant predictor of a related form of social support, namely reduced social integration. Social integration relates to a sense of "belonging" which is derived from membership in a group of individuals with similar interests, concerns, and/or recreational activity. This type of support, also known as "network support" or "belonging support," is most often provided by friends. Those with greater communication difficulty had a significant reduction in this type of support. 
Further insight into the reason for this finding was provided by comments from the qualitative interviews. Interview participants tended to emphasize the continuity of all of their social relationships, including those with friends and family over time. Most participants reported going to great lengths to maintain friendships over long periods and distances. Some individuals indicated a reduced interest in group membership with individuals that they did not feel a close connection with. Despite this, the importance of companionship and of maintaining social connections was generally agreed. Although family sometimes took the place of friends, the importance of close friendships (people who "look at the world like I look at it") was acknowledged by almost all. Some changes were reported to occur, however. Due to increased reliance on a spouse or partner, for example, some individuals reported spending more time with "couples who are friends," being "out of the loop" with regard to planning social events, and having less control over who they socialized with. Some life transitions resulted in a disproportionate loss of friends. Relocation and retirement, for example, sometimes resulted in the sudden loss of "close friends and acquaintances." Some members of the social network also "fell by the wayside" following the diagnosis of a disability. There also appeared to be a change in the ability to make new friends for some participants, even when they were in social contexts there were many opportunities for doing so. This was exemplified by the participant who was puzzled at the fact that she had not made a connection with anyone that she would "consider a close friend" during the three years she had resided at a retirement community. Consequently the loss of friends appeared to occur for a variety of reasons including the loss of social roles, changes in the social context, and difficulties 
with social participation, but the loss of friends was not a desired change on the part of most individuals.

\section{Communication impairments were associated with reduced frequency of} social participation and a reduction in the number of social activities. After controlling for demographic, health, and disability characteristics, communication was a significant independent predictor of a reduced frequency of social participation and a reduced number of social activities. Interview participants reported difficulties in a wide range of social contexts such as cocktail parties, loud restaurants, communal dining rooms, festivals, and public events. In some cases, difficulties being heard and understood made participation in social events less rewarding. In other cases, the environmental demands of some social situations were too challenging. Noise was annoying or even painful for those with hearing loss. Those with voice problems experienced physical discomfort in trying to make themselves heard above it. The difficulties that they experienced, particularly in group settings, often resulted in feelings of wearing an "invisibility cloak" or being "alone in a crowd." For most people, however, there were reasons to continue social participation. Even though they found participation in some activities less rewarding than they had in the past, some individuals continued to attend in order to maintain their social connections. Others "made the effort" on behalf of their spouse or partner. In some cases individuals went to great lengths planning their participation in social events, including choosing in advance who to go with and how long to stay. 
In general, social isolation was rare in the current study and occurred in only $12 \%$ of the sample, which is consistent with other estimates from studies of older adults. Individuals who were socially isolated had poorer communication scores bordering significance. Complete social withdrawal was rare but did occur. One participant succinctly described his progression to becoming socially isolated from being "alone in a crowd" until finally: "You just end up being alone off by yourself and do things just by yourself." This individual had been deafened in an explosion and experienced a severe noise-induced hearing loss. This individual had one of the most severe communication impairments among those interviewed, suggesting that those with the most severe communication impairments may be at greatest risk of social isolation and withdrawal.

There was no evidence of an interaction between communication impairment and functional limitations with regard to psychological well-being. In the analysis of data from Phase 1 of the study, no evidence was found for an interaction between communication impairment and functional limitations in predicting either loneliness or depression. During interviews with study participants in Phase 2, it appeared that communication impairments were perceived to be less disturbing when they co-occurred with other limitations. For some individuals this was accepted as part of a normative process of aging: "We're getting old!" For others the changes were accepted as being part of a disease process, as with the progressive changes resulting from Parkinson's Disease: "I take each change as it goes along." In contrast, far greater disruption occurred when there was a sudden change in functional ability, such as after a stroke, accident, or surgery. One example of this was the study participant who experienced a stroke and felt 
that "my identity had left me because I couldn't do what I used to do." A similar level of disruption was reported by individuals who experienced a mismatch between their communication and their physical abilities, even though this may have been gradual in onset. For individuals who were physically healthy and active, the development of a communication impairment caused a disruption in their social roles and responsibilities. Some individuals relinquished those roles voluntarily because they felt that they could no longer perform these roles "adequately," while others experienced the "shock" of a "forced retirement" or being classified as "disabled" by others.

\section{Communication impairment was not associated with an increase in negative}

social interactions. Communication impairment was not a significant predictor for higher levels of negative social interactions in the data from Phase 1. During the interviews in Phase 2, however, negative interactions were commonly reported. Participants described interpersonal strain between themselves and their spouses as a result of communication impairments and also provided examples of miscommunications and negative comments during conversations with familiar and unfamiliar adults. In general, however, respondents appeared adept at insulating themselves from conflict and criticism. When negative reactions occurred, however, participants were very sensitive to their occurrence. Participants reported apologizing in advance for their deficits, reacting angrily to or being hurt by insensitive comments, and using a spouse as an intermediary when communication breakdowns occurred. In some cases, they abandoned attempts at communication altogether: "I have noticed the diminution of my social communication. It's easy to be quiet and just wait till you know somebody really wants to talk to you." 
Consequently the lack of an association between communication impairments and negative interactions in Phase 1 may mask a more concerning process, namely the efforts that individuals with communication impairments make to insulate themselves from situations where negative interactions might occur. This possibility is explored in greater detail in the Discussion.

There was an interaction between communication impairment and social self-efficacy in predicting social network size, but the nature of this interaction was

contrary to predictions. It had been hypothesized that those with higher levels of "social skill" would be able to compensate more effectively for the presence of a communication impairment and maintain a larger social network. For those who were "less skilled" it had been anticipated that they would be less able to compensate and communication impairments would be more strongly associated with network size. The quantitative data from Phase 1 suggested the opposite. Communication was associated with social network size for those with medium and high levels of social self-efficacy. For those with the lowest levels of social self-efficacy, however, there was no significant relationship. This finding proved somewhat difficult to interpret because it was not consistent with the original hypothesis of social self-efficacy as a measure of "social coping" or "social control." Alternative explanations for this finding are explored in the Discussion.

Even though individuals for Phase 2 had been selected for this very purpose, the interviewees provided relatively few insights into the concept of social self-efficacy. During questioning, very few participants spoke consciously about the need to negotiate support-related needs, nor about ways in which they were able to compensate for 
relationship losses, with one notable exception. This participant, who experienced disabling medical conditions during middle adulthood, had consciously cultivated a network of "emergency contacts" and spoke insightfully about the steps she had taken to do so. She described how she had created her own support network of "emergency contacts" made up of "younger friends" who "all have some kind of chronic illness" that could provide assistance to one another, as needed. She appeared to be atypical in this respect, however, perhaps because she had been dealing for disability for a much longer period, or because she also had to deal with poverty and a lack of family support. Other interview participants did not report similarly conscious efforts directed towards network development and maintenance. Some additional insight was provided by analysis of some of the open-ended questions from the study sample in Phase 1. After completing the social self-efficacy survey, respondents were asked whether they felt that these characteristics had changed over time. The majority of respondents $(62 \%)$ reported no changes in their social self-efficacy over time. Of those who reported changes, changes in communication were the most commonly reported reason.

\section{Communication impairments were associated with increased loneliness and}

\section{depression with different pathways for each, involving social self-efficacy and}

reassurance of worth. Findings supported two distinct pathways for loneliness and depression. Communication was a significant predictor of loneliness after controlling for the mediator, social self-efficacy, consistent with partial mediation. For depression, however, communication was no longer a significant predictor after controlling for the mediators social self-efficacy and reassurance of worth, consistent with full mediation. 
Interview participants provided numerous examples of how communication impairments had changed their perceptions of themselves and also how they were treated by others. Some individuals perceived themselves differently as a result of their communication changes. Participants tended to judge themselves as appearing less "confident" and "interesting," felt "more self-conscious" and described a loss of a sense of self. Role losses secondary to no longer being able to perform "adequately" or assume "leadership positions" were coupled with societal messages reinforcing a sense of irrelevance, such as taking "forced retirement" or being signed up for disability despite a desire to continue working. Together, these experiences appeared to have taken a toll on self-perceptions of competence and worth and often associated with considerable psychological distress. 


\section{Discussion}

\section{Study Contributions}

The current study investigated the relationship between health, functional disability, and communication impairments on a range of social measures. A mixedmethods study design was employed. Community-dwelling older adults were surveyed about the size and diversity of their social networks, frequency of social interactions, and physical and mental health. Qualitative data were also collected from a smaller subsample. Specific aims of the study were to examine whether communication impairment was associated with social measures, whether there was an interaction between communication impairments and physical disability, and to examine the role of relationship control strategies in maintaining access to a larger or more supportive social network. To date, most large-scale studies of older adults have examined the impact of hearing impairment but have not considered a more general definition of communication impairments in their predictive models. This study is one of the first to examine the impact of communication impairments on a wide range of social measures in older adults.

There is a significant body of evidence that the quantity and quality of an individual's social relationships are associated with better physical and mental health across the life-course (Holt-Lunstad et al., 2010; House et al., 1988). Social support has been shown to buffer stress, promote better psychological well-being, and reduce the risk of disability, morbidity, and mortality (Berkman \& Glass, 2000; Cohen, 2004; Uchino et al., 1996). Social support is also associated with the risk of hospitalization and institutionalization (Tobin \& Kulys, 1981). In studies of older adults, psychological 
outcomes are associated not just with the size but also the composition of the individual's social network (Fiori et al., 2006). Findings from these studies attest to the importance of social relationships for positive mental health and well-being.

Previous research suggests that adults with communication impairments may be at-risk for poorer psychological well-being, with higher levels of loneliness and depression and reduced life satisfaction (Hawthorne, 2008; Kramer et al., 2002; Parr, 2007; Strawbridge et al., 2000; Weinstein \& Ventry, 1982; Yorkston et al., 2010). There is also evidence that communication impairments may have a deleterious effect on social support and social networks over time, placing individuals at increased risk of isolation (Pachana et al., 2008; Schneider et al., 2010).

The findings from the current study supported an association between communication impairments and several important aspects of social relationships. Communication impairment was associated with a significant reduction in the number of relationships with friends, a reduction in certain aspects of social support, reduced social participation, and a decline in social self-efficacy. Communication impairment was also a significant independent predictor of greater loneliness and depression, and reduced social self-efficacy. In terms of psychological health, no evidence was found for an interaction between communication impairment and social support, nor between communication impairment and physical disability. Mediation analyses were also used to investigate the relationship between communication and psychological well-being. Findings supported two distinct pathways for loneliness and depression. Communication was a significant predictor of loneliness after controlling for social self-efficacy, consistent with partial 
mediation. For depression, however, communication was no longer a significant predictor after controlling for social self-efficacy and reassurance of worth, consistent with full mediation. Taken together social self-efficacy and reassurance of worth appear to be important in explaining the connection between communication and psychological wellbeing. The implications of these findings are discussed in greater detail below.

\section{Interpretation of Findings}

This section will discuss how these findings relate to the study hypotheses and also the relevance of these findings to theory.

\section{Communication impairments were associated with having fewer friends in} the social network and a reduced sense of social integration. It had been anticipated that communication impairment would be a significant predictor of overall social network size, but this was not the case. Instead, the study findings appeared to show that communication impairment disproportionately affected relationships with friends. The absence of friends from the social network did not appear to be deliberate. In some cases the loss of friends was associated with life transitions such as relocation and retirement. This may have been exacerbated by a reduced ability to replace network loses and make new friends. In addition reduced social participation (discussed below) may have reduced the number of opportunities to sustain current friendships or develop new ones by meeting "people who were likely to be my friends." As a result, there was an increased tendency to rely on family in place of friends. The loss of friends was not a desired change in most cases, however, as indicated by the reduction in the sense of social integration or "belonging support." 
These findings are consistent with previous research into the impact of aphasia following a stroke, which have demonstrated that older adults with aphasia have smaller social networks with fewer friends (Davidson et al., 2008; Hilari \& Northcott, 2006). Previous research on older adults with other kinds of disability has also demonstrated that the presence of a disability is associated with an increase in the number of kin in the social network and a smaller number of friends (Mugford \& Kendig, 1987). Findings from the qualitative portion of the study provided additional insights. Some interview participants indicated that they were less interested in participating in group activities with other people that they did not feel a strong connection with. A lack of interest in superficial social relationships consistent with the principles of Socioemotional Selectivity Theory, according to which older adults choose to maintain social relationships that are most rewarding and gradually abandon social relationships which are less rewarding (Carstensen et al., 1999). This lack of interest was not shared by all participants, however. Many reported that their friendships were just as close as they had always been and that they invested significant efforts in maintaining them over long periods and, often, long distances. This type of network maintenance and continuity has previously been described as the Convoy Model (Kahn \& Antonucci, 1980), in which the individual maintains their part of "convoy" of individuals over the life-course. Other factors that appeared to be involved in the loss of friends from the social network included relocation, retirement, health changes, relying on a spouse for greater access to the social network, and greater difficulty making new friends. 
The fact that smaller, family-based social networks might be less satisfying was also exemplified by the interview participants. Cornwell (2011) has argued that while close family networks may provide a sense of "embeddedness" and access to social support, they may also provide obstacles to autonomy and privacy. He hypothesized that it may be important for older adults to maintain "bridging potential" in their social networks or, in other words, ties to other adults who are otherwise poorly connected to each other. This concept is similar to Granovetter's theory of the "strength of weak ties" (1973) which posits that more casual acquaintances outside an individual's social circle might provide access to information, companionship, and other resources that are not otherwise available. In the current study, when a partner formed a "bridge" to the rest of the social network participants reported a sense of being "out of the loop" with social planning which, in turn, resulted in feelings of being "less in control and less involved."

Such findings are not consistent with the changes in social networks described by Socioemotional Selectivity Theory, as demonstrated by the fact that individuals with smaller social networks had higher levels of loneliness and depression, suggesting that smaller social networks were associated with lower (not greater) levels of overall satisfaction. In addition, some individuals emphasized the importance of relationships with those who were less well-known and less familiar to them, which is also inconsistent with Socioemotional Selectivity. Support groups were a vital link to creating a new social network and interactions with other individuals in the support group were more satisfying in a number of ways because individuals were accepted and treated as peers in that context. It appears, therefore, that Socioemotional Selectivity Theory may describe some 
of the social changes associated with normal aging but does not account for all of the social needs of older adults, particularly those with a disability. Close friends and family members may react in unwelcome ways to the changed appearance or abilities of the individual, whereas newer friends, acquaintances and less well-known peers might not. This is also consistent with other work that has shown that friends seem to be particularly important for feelings of emotional well-being and self-esteem (Johnson \& Catalano, 1983; Lee, 1979; Rook, 1987; Wood \& Robertson, 1978), whereas family may be more important for providing practical and material assistance (Tobin \& Kulys, 1981).

It had been predicted that communication impairment would be a significant predictor of reduced social support based on previous findings in the literature, but this proved not to be the case. Instead, communication impairment was associated with lower levels of two particular functions of social support, social integration and reassurance of worth. The first of these, social integration, relates to a sense of "belonging", which is derived from membership in a group of individuals with similar interests, concerns, and/or recreational activity. Social integration, also known as "network support" or "belonging support," is most often provided by friends. The reduction in this type of support is consistent with the fact that there was a significant reduction in the contribution of friends to the overall social network. This finding reinforces the importance of friendship. Further, it suggests that the psychological benefits conferred by friends cannot be easily replicated by family members, as has been shown in other research (Felton \& Berry, 1992; Fiori et al., 2006; McIlvane \& Reinhardt, 2001). 
Communication impairment was associated with reduced frequency of social participation and a reduction in the number of social activities. Communication impairment also predicted the frequency of social participation and the number of social activities in which the individual participated on a regular basis, as had previously been predicted. Despite the difficulties that they experienced as a result of their communication impairments, most participants continued to enjoy the company of others and were active in a wide range of social situations. In addition to family activities, they continued to socialize with friends, volunteered, attended religious worship, took classes, and regularly attended group activities over the course of many years. The reduction in social participation, therefore, appeared not to be the result of a lack of interest in social contact. Instead, many individuals limited their participation in certain kinds of social activities due to communication challenges in those contexts.

Social isolation was rare in the current study and occurred in only $12 \%$ of the sample, which is consistent with other estimates from studies of older adults. Individuals who were socially isolated generally had poorer communication scores but a larger sample would be required to examine this association definitively. Social withdrawal, indicating a loss of interest in social contact or an inability to interact with others in a meaningful way, was rare and occurred only for those with the most severe communication difficulties.

The association between communication impairment and social participation was consistent with findings from the preliminary investigation for the study (Palmer et al., 2012) but differed from some other research. In studies of community-dwelling older 
adults, communication-related measures have not been predictive of social participation (Cruice et al., 2005). In contrast, studies of clinical populations have demonstrated associations between communication impairment and participation (Baylor et al., 2010; Mulrow, Aguilar, Endicott, Tuley, et al., 1990). Reasons for the inconsistencies in findings between the studies may include differences between the study populations and the study measures used. In particular, the participants in the study by Cruice and colleagues (2005) were generally healthy and had a low incidence of communication difficulty on the screening measures they employed. The data from the current study, however, provide compelling evidence that social participation is negatively affected by communication impairment. It should be emphasized that most participants continued to enjoy the company of others and were active in a wide range of social situations. The reduction in social participation, therefore, appeared not to be the result of a lack of interest in social contact. There are, therefore, two possible explanations for this finding, both of which may be involved to varying degrees. The first explanation is that individuals limited their participation in certain kinds of social activities due to the challenges associated with those contexts. This would be consistent with the principles of Selective Optimization with Compensation. The second explanation is that individuals were noted to withdraw from situations in which conflict or negative interactions occurred. Participants were apparently successful at insulating themselves from these types of occurrence but may have done so at the risk of social isolation.

There was no evidence of an interaction between communication impairment and functional limitations with regard to psychological well-being. Previous research 
had suggested that certain types of variables may interact with each other with regard to psychological well-being. Kempen and her colleagues (1998) found that hearing impairments had independent and unique predictive value with regard to different types of limitations. In addition to these main effects, hearing losses exacerbated the impact of other types of impairments on disability measures, supporting the hypothesis that sensory impairments might interact with physical limitations. This finding was not replicated in the present study. It is unclear whether this inconsistent finding might be due to the differences in study measures utilized. Another explanation suggested by the interviews with study participants, however, was that often communication impairments were perceived to be less disturbing when they co-occurred with other limitations. For some, the co-occurrence of limitations was accepted as part of a normal process of aging. For others, these changes were accepted as being a typical part of a disease process, such as with Parkinson's Disease. Slow changes in physical ability and communication may have allowed time for adaptive changes, psychological adjustment, and less overall disruption. This is consistent with theories such as Person-Environment fit, as well as theories of coping and adjustment, and Selective Optimization with Compensation.

In contrast, the onset of sudden changes was much more disruptive, such as after a stroke, accident, or surgery. It was also noted that situations in which there was a greater mismatch between communication and physical ability caused greater disruption, even when it was slow in onset. Some evidence from the literature about adult-onset disability would support this hypothesis. In a study of couples in which one partner had been diagnosed with multiple sclerosis (MS; Starks et al., 2010), the authors identified two 
patterns of adaptation. Couples that were "in sync" were characterized by a relapsingremitting type of MS that progresses more slowly, allowing both partners to maintain their social roles and identities, and that this allowed for the development of a more collaborative problem-solving style. Couples that were "out of sync" had a more rapid progression of MS, which was associated with the loss of employment before retirement age, and struggles with preadolescent children. This type of disruption is also consistent with research about other adult-onset disabilities, such as visual impairment, that has shown that middle-aged adults experience more disruption than older adults (Boerner \& Wang, 2010). The higher number of problems reported by middle-aged adults may be due to greater complexity of their responsibilities, including work, marital, and childrearing responsibilities, and also to the earlier onset of a disability being considered an "off-time event." It can be hypothesized that a communication impairment occurring in a working older adult may, therefore, be more disruptive than the co-occurrence of both communication and physical disabilities in an older adult who may have retired and relocated as a result of health changes.

\section{Communication impairments were not associated with an increase in}

negative social interactions. It had been hypothesized that the severity of communication impairment would not be an independent predictor of negative social exchanges. Communication impairments have been shown to have negative impacts on personal relationships including declines in marital intimacy and satisfaction, and increases in frustration, interpersonal strain, anger, resentment, and blame (Baikie, 2002; Carter, et al., 1998; Hétu et al., 1993; Joubert et al., 2011). Older adults are known to 
seek to insulate themselves from relationships that are unrewarding or problematic. According to Strength and Vulnerability Integration theory older adults are more susceptible to the physiological impact of stress and will work harder to avoid or prevent conflict in their interpersonal relationships (Almeida et al., 2011; Charles, 2011). In the preliminary work for the current study, there was no significant association between communication impairments and negative interactions (Palmer et al., 2012). This finding was supported in the current study. During interviews, participants described interpersonal strain between themselves and their spouses as a result of communication impairments. They also provided examples of over- and under-accommodation during conversation with friends and family, consistent with Communication Accommodation Theory. In general, however, most participants appeared adept at insulating themselves from conflict and criticism. It is possible that older adults who experience negative social exchanges may be able to limit their exposure to these types of interactions through social withdrawal (Morgan, 1989), which would result in less frequent negative social exchanges. Avoidance of negative interactions might, in turn, be one of the factors responsible for the reduction in aspects of the social network and also reduced social participation. It is possible, therefore, that in order to protect themselves from upsetting social interactions, older adults with communication impairments may place themselves at greater risk of social isolation.

\section{Communication impairment as a risk-factor for reduced social support.}

Previous studies had found that individuals with communication impairments were at-risk for reduced social support (e.g. Pachana et al., 2008; Schneider et al., 2010). This finding 
was not replicated here. It was also not possible to explore Cantor's $(1979,1980)$ "hierarchical compensation model" in which more distant bonds (e.g., nonkin) become relatively more important when closer bonds (e.g., kin) are unavailable. In this model, functions are more likely to be transferred than lost and there is an explicit ordering of responsibility from spouses and children to other relatives, friends and neighbors. Due to the general availability of family support, it was not possible to examine this hypothesis. In fact, very few participants spoke about consciously negotiating their support-related needs or ways in which they were able to compensate for relationship losses, with one notable exception. This participant, who experienced disabling medical conditions during middle adulthood spoke eloquently about how she compensated for her disabilities and also negotiated access to a support network. In doing so, she demonstrated very adaptive principles consistent with theories of stress and coping. She was an advocate for her needs and educated others about the impact of health conditions. She also consciously planned her social participation, including what types of activities to participate in, for how long, and who to attend with, which is consistent with Selective Optimization with Compensation. Most other study participants did not report consciously using these types of coping strategies. It is possible that this was because many of the study participants were married or had supportive family and so had not needed to recruit additional support. It is also possible that these types of behaviors may not be typical of older adults in general. The individual who described creating a support network of "emergency contacts" made up of "younger friends" had been disabled during adulthood, had limited finances, and limited family support. The combination of these factors may have forced 
her to develop more active coping strategies over a longer time period. For others, these types of strategies for consciously meeting support needs or negotiating social situations may not have been required.

The absence of a "buffer effect" for social support. Previous research had also suggested that social support might "buffer" the impact of communication impairment on psychological well-being. According to these studies, individuals with a communication impairment and low levels of social support are at risk for greater levels of mental distress. In this study, however, there was no evidence of an interaction between communication impairment and social support with regard to either loneliness or depression. Some previous studies have reported an association but the findings have been inconsistent, perhaps due to differences in methodology and measurement. In addition previous studies may have lacked a sample size large enough to determine significance (McClelland \& Judd, 1993). In their study of community dwelling older adults, Oppegard and colleagues (1984) found that hearing impairment was significantly correlated with anxiety and depression, but only for those with low levels of social support which supported the "stress-buffer" hypothesis. The authors did not use a published social support measure. Instead they used a measure of how often study participants saw children and family, which might indicate the size of the family social network, its emotional closeness, and the potential availability of support, but is less comprehensive than other published measures. In addition, the study did not control for any other confounding variables between the two groups, such as demographic characteristics, health, or disability. Other studies have reported associations between 
communication, social support, and well-being using well-validated measures but have not controlled for other factors in the analysis (Blood et al., 1994; Frankel \& Turner, 1983). The current study differs in that a multiple regression model was used in order to control for demographic characteristics, health, and disability and therefore examine the contribution of social support independent of these other factors. The current study found no evidence of social support as a moderator between communication impairment and psychological well-being. In addition, individuals with communication impairments appeared to be getting their support needs met, with the exception of the two types of social support described previously.

\section{Communication impairments were associated with increased loneliness and}

\section{depression with different pathways for each, involving social self-efficacy and}

reassurance of worth. Several analyses were conducted to examine which of the social relationship variables associated with communication impairment might, in turn, predict psychological well-being. Findings supported two distinct pathways for loneliness and depression. Communication was a significant predictor of loneliness after controlling for a mediator, social self-efficacy, consistent with partial mediation. For depression, however, communication was no longer a significant predictor after controlling for the mediators social self-efficacy and reassurance of worth, consistent with full mediation. It appears that social self-efficacy and the reassurance of worth may be important characteristics for understanding the relationship between communication impairments and psychological well-being. 
Reassurance of worth (or "esteem support") has not been highlighted in this type of analysis previously. The items on this subscale asked respondents to agree or disagree with statements about being regarded as competent and respected or admired for their talents or abilities. Some previous research has shown that individuals with communication impairments feel less confident in their own abilities and may also be treated as less competent by others (Babbitt \& Cherney, 2010; Baylor et al., 2011; Marsiske et al., 1997). During the qualitative interviews, there were numerous examples of why this might occur. Communication impairments left individuals feeling as though they could no longer perform "adequately" or "contribute in the manner I was used to." As a result, many experienced voluntary or involuntary retirement, disability, and the relinquishment of positions of authority. These role losses and the inability to participate in key activities that had been central to their self-concept was associated with significant disruption. As a result of communication changes, participants reported that those around them treated them differently, reinforcing a sense of incompetence and disability. Some participants reported that spouses would sometimes act as an intermediary when communication breakdowns occurred. In other cases, individuals reported that conversational partners "said the word for me" even though "it wasn't what I was thinking!” These findings are also consistent with the qualitative study by Baylor and colleagues (2011) in which participants described having to use alternate methods of communication, adapt their method of communication, rely on others to communicate for them, and/or ask for accommodations from their communication partners. In some situations, these strategies or accommodations were not effective and this resulted in 
withdrawal from a variety of social situations well as life roles and positions of responsibility, including those related to work, group membership, and community involvement. As a result, Baylor's participants described feelings of isolation and marginalization, of feeling "like a bystander," "out of the loop," or "ignored," as well as feeling as though they had lost their "sense of self" (pp.275-276) very similar to those described in the current study. In addition to the reactions of others, study participants also reported that they perceived themselves differently as a result of their communication changes. These feelings included a loss of "authority" and "interest," being more "self-conscious," and having to rely on others to communicate for them in certain situations. Such comments are consistent with previous research into the association with communication impairments and feelings of mastery, control, confidence, competence (Babbitt \& Cherney, 2010; Baylor et al., 2011; Marsiske et al., 1997).

The significant relationship between communication impairment and social selfefficacy is also a novel finding. As conceptualized in Bandura's Social Cognitive Theory, self-efficacy is the conviction that one can successfully execute the behavior required to produce the outcomes $(1977,1986,1997)$. Self-efficacy perceptions are domain-specific, indicating an individual may have high self-efficacy for the skills associated with one type of activity but low self-efficacy for other domains of activity. In addition, selfefficacy is not a fixed personality construct but changes as a result of a wide variety of life experience and can be influence by mastery experiences, vicarious experience, verbal persuasion and physiological and affective states. With regard to social self-efficacy, 
some authors have argued that older adults employ "proactive aging" in order to negotiate relationship losses and meet their emotional and physical needs (Cantor, 1979, 1980; Lang \& Carstensen, 1994). In addition the "support-efficacy model" has also been proposed in which it has been hypothesized that self-efficacy may help to explain the association between social relationships and well-being (Antonucci \& Jackson, 1987). According to these theories older adults may be able to maintain their social network and its associated support through relationship control and management activities at the individual level, such as through social self-efficacy. Consequently, social self-efficacy was examined as a possible moderator between communication impairment both the size of the social network and also the amount of social support that the individual received to determine if this might be true.

\section{There was an interaction between communication impairment and social}

self-efficacy with regard to social network size, but the nature of this interaction was contrary to predictions. There was no evidence of an interaction between communication impairment and social self-efficacy for social support. There was, however, evidence of an interaction between communication impairment and social selfefficacy with regard to social network size. This finding proved somewhat difficult to interpret because it was not consistent with the original hypothesis of social self-efficacy as a measure of "social coping" or "social control." As depicted in Figure 11, there was a stronger association between communication and social network size for individuals with high social self-efficacy. If social self-efficacy is conceptualized purely as a measure of relationship control, this finding appears nonsensical. Individuals with higher levels of 
"social skill" ought to be able to compensate better for the presence of a communication impairment and maintain a larger social network than those that are "less skilled." As suggested by Figure 11, however, the opposite would appear to be true.

One possible solution to this paradox is to consider an alternative relationship between the three variables in question. It had been hypothesized that social self-efficacy was a moderator between communication impairment and social network size. An alternative hypothesis would be that social network size is a moderator between communication impairment and social self-efficacy. In this model, individuals with more severe communication impairments who have a larger social network are exposed to more negative reactions or situations in which they may judge their social performance more negatively. As a result of these negative interactions there is a decline in social selfefficacy over time. According to Bandura, performance accomplishments are the most powerful source of efficacy expectations (1977). As such, self-perceived failures have a particularly deleterious effect on self-efficacy, particularly if they are repeated. "Successes raise mastery expectations; repeated failures lower them, particularly if the mishaps occur early in the course of events" (p.195). Hypothetically, then, it could be speculated that individuals with a larger social network and more significant communication impairments might experience more "repeated failures" resulting in reduced social self-efficacy. Support for this hypothesis is provided by Babbitt and Cherney's (2010) work on "communication confidence," based on the experiences of individuals with aphasia. They hypothesized that confidence in the ability to communicate may be strongly associated with the constructs of personal autonomy, self- 
efficacy, and self-determination. When an individual's interactions with others prove problematic due to the presence of a communication impairment, this led to decreased confidence in the ability to communicate personal wishes, diminished autonomy, and learned helplessness. Further support for this interpretation is provided by other recent research based on interviews with individuals with communication impairments secondary to a wide variety disorders (Baylor et al., 2011). During the interviews, the participants were asked about factors that caused interference with communication during their daily lives. The concept of "communicative interference" is relatively novel but the researchers found that when they asked questions about interference with communication "seemed to resonate with the study participants in that they readily had many examples of experiences, and many already had terms that they used to frame their thinking and describe their experiences" (p. 280). Interpreting the findings in Figure 11 using the perspective of "communicative interference," one possible explanation is that those with a large social network (which likely includes more friends and also greater social participation) and greater levels of communication difficulty experience greater "interference." Communication for this group would be associated with greater levels of social discomfort, less pleasure in social settings, and less self-confidence in public as a result. For those with a smaller social network, the fact that they interact more with family-members rather than friends and avoid more social situations/interactions means that they do not experience this same level of "interference," regardless of the severity of their communication impairment. This hypothesis might also explain the mechanism underlying social withdrawal on the part of individuals with communication impairments. 
Social withdrawal may be used as a way to lessen the frustrations, discomfort, and interference associated with social interaction.

\section{Communication impairment was the only significant predictor of social self-}

efficacy. One additional surprising finding was that communication was the only significant predictor of social self-efficacy. This was consistent with the finding from the

open-ended responses in which communication was the most commonly listed reason for negative changes in social self-efficacy. Further insight into the nature of social selfefficacy was provided by the open-ended responses from the Phase 1 participants. After completing the social self-efficacy survey, respondents were asked also whether they felt that these characteristics had changed over time. The majority of respondents $(62 \%)$ reported no changes in their social self-efficacy over time. Of those who reported changes, respondents felt that their social self-efficacy was influenced by a range of other factors including reduced shyness (or its opposite), relocation, health, as well as changes in social skill. As such the social self-efficacy measure appeared to be affected by changes in personality-related characteristics (e.g. introversion, self-esteem, and sociability), social priorities (e.g. how pleasurable an individual finds social interaction), as well as characteristics of the social environment (e.g. proximity to friends and opportunities for daily interaction with others), and changes at the individual level (e.g. health and communication). Another insight from the work of Baylor and colleagues (2011) may also be used to understand the nature of the concept of social self-efficacy, and how it might be affected by communication, more generally. The researchers found that interference appeared to have two components: 
One aspect of interference was restrictions in the ability to accomplish or engage in tasks, and the other component was a range of largely negative emotions about their experiences. When talking about the causes of interference, participants identified variables over which they felt they had little or no control (e.g., health symptoms and environmental factors), as well as their own reactions and self-imposed decisions that shaped their participation (p.280).

The dual aspect of interference can be compared to the concept of Person-Environment fit in which competence is neither a function solely of the individual, nor of the environment. Competent behavior occurs when the abilities of the individual match the demands and resources of the environment. This type of duality may also be relevant to understanding the changes in social self-efficacy in that individuals described changes as a result of both situational (e.g. factors related to health and the environment) as well as internal changes (e.g. changes in sociability and self-confidence). The fact that interference may be caused by a variety of different factors is also consistent with other qualitative research with individuals with disabling conditions that cause physical as well as communication impairments. In one study, participants emphasized that communication is just one "part of the picture" and needs to be considered in the context of both health changes and the social context (Walshe \& Miller, 2011). If so, then social self-efficacy may be a marker of the congruence between the individual and their environment. As such it could be used as a measure of adaptation and, potentially, an indicator of the success or failure of interventions at either the individual or the environmental level.

A number of previous studies have shown that, in older adults, the presence of a hearing impairment is a significant independent predictor of reduced self-efficacy, 
control and mastery even after controlling for health and demographic characteristics. Kramer and colleagues (2002) found that hearing impaired people reported more depressive symptoms, lower feelings of self-efficacy and mastery, more loneliness, and a smaller social network than their normally-hearing peers. Ormel and colleagues (1997) found that the presence of a hearing impairment was associated with higher levels of physical and role disability, and with lower levels of mastery, self-efficacy, and social support. Unlike other chronic health conditions, for hearing impairment, the mental health effects appeared to be carried forward by reduced mastery and self-efficacy only. In explaining this relationship, the authors suggested that hearing impairments not only limit participation in various kinds of activities but also lead to declines in the sense of control, competence and self-confidence which, in turn, cause increased distress, anxiety and depression.

The findings from the current study would suggest that similar types of processes occur in older adults with a wide variety of communication impairments, not just those associated with hearing impairment. Although the concept of general self-efficacy was not measured directly, it is likely that the social self-efficacy measure provides an insight into more global sense of mastery and control and this was confirmed by comments from the interviews. This is also reinforced by the fact that the reassurance of worth was another key variable, suggesting the importance of feeling valued, respected, and regarded as competent. These findings are consistent with other research which has shown that communication impairments predict significant reductions in perceived competence with basic activities of daily living and feelings of perceived control, 
competence, and self-confidence (Babbitt \& Cherney, 2010; Kramer et al., 2002; Marsiske et al., 1997; Ormel et al., 1997).

\section{Limitations of the Study}

There were a number of limitations to the current study. The study sample was predominantly White and non-Hispanic and was less diverse than estimates for both the U.S. population and those in the Pacific Northwest. This limits the generalizability of the findings and reinforces the need for more research with diverse samples of older adults. In addition, most individuals were recruited through a medical center or through support groups and organizations that provide information and access to resources. Many of the study participants can therefore be assumed to have had some access to information, treatment, and rehabilitation. It is possible that current study underestimates the impact of certain health conditions and communication impairments by underrepresenting those who have not received appropriate care and treatment for these problems.

The study was cross-sectional. As a result, it is not possible to definitively examine the extent to which changes in social relationships are the effect of cumulative factors occurring over the life-course, nor the ways that communication impairments may vary in their impact based on age of onset or their duration. Detailed longitudinal data would be needed for such an analysis. Further, the fact that the current study is crosssectional implies that causal relationships between health, communication, social relationships and well-being cannot be proved from the study findings.

One of the strengths of the study was that the instruments used in the survey were published, validated measures that had previously been used in research with older adults. 
To date, some of the instruments have not been used widely with older adults that have communication disorders, however. This study demonstrated good reliability for the majority of the measures, which is one of the study's contributions. Surprisingly, there is no single measure of functional communication that has been validated and used across a wide variety of communication disorders, including those relating to voice, speech, and hearing. The CETI-M has been previously validated for some of these groups and demonstrated good face validity with other measures. Nonetheless, the need for a validated generic measure is one suggestion for future research.

It should be emphasized that the study design deliberately over-sampled individuals with various communication disorders in order to increase the statistical power of the analysis and to determine what effect, if any, communication might have on social relationships. As a result, the data cannot be considered "typical" or normative and therefore cannot be used to provide estimates of incidence or prevalence. Surprisingly, however, given the nature of how the study sample was recruited, comparisons of the study data with other previously published studies showed remarkable similarities (Appendix K).

\section{Clinical Implications and Suggestions for Future Research}

Based on the study findings, a number of recommendations can be made with regard to future research as well as clinical practice. First, it does appear feasible to conduct studies of older adults with a wide variety of communication impairments generally. Although most published instruments published to date are disorder-specific, it does appear that adapted versions of functional measures of communication such as the 
Communicative Effectiveness Index-Modified (CETI-M) could be valid for use in wider populations. This conclusion should be validated by future research with the goal of providing more participation-based instruments that can be used across a variety of disorders.

Second, the study findings suggest that current conceptualizations of communication disorder severity may provide little insight into the disruptiveness of a communication disorder on an individual's daily life and function. Typically the severity of a communication disorder is classified into categories of "mild," "moderate," and "severe" based on objective criteria. These criteria are often used as a guide for the urgency and nature of intervention efforts on the part of the clinician, sometimes supplemented by a subjective measure of communication-related handicap or quality of life. Interviews with study participants confirm, however, that a third perspective might be more beneficial, namely that of Person-Environment fit. For example, number of individuals with relatively "mild" communication problems were forced to give up positions of authority or leadership because they could no longer function adequately in that context, resulting in significant disruption to their daily lives as well as personal distress. In contrast, other individuals with more severe communication problems were less disturbed because their communication abilities were more suited to their daily needs. Using a Person-Environment Fit perspective towards the classification of a communication disorder (i.e. the level of mismatch between an individual's communication ability, their communicative demands, and the communication context) 
might better help to guide treatment and rehabilitation efforts in a manner that is most meaningful to the individual.

Third, it appears that findings from previous research regarding the impact of a hearing impairment on the perceived competence and self-confidence of older adults can be generalized to older adults with communication impairments generally.

Communication impairments may have a particular impact on social self-efficacy and the sense of self-worth and these may be key factors in explaining the association between communication impairments and psychological well-being. Placed in the context of previous work on communicative interference it is possible that those with more frequent social contact and poorer communication abilities experience greater interference and this, in turn, may explain the process of social withdrawal. These hypotheses remain for further investigation. In the current study social isolation was rare and a much larger sample would be required to examine risk factors for isolation definitively.

In addition, future research should be guided towards understanding the impact of different types of rehabilitation with self-efficacy measures as indicators of treatment outcomes. The application of a self-efficacy framework has been described in the field of audiology (Smith \& West, 2006), but self-efficacy has rarely been described as a therapeutic target for communication disorders. Using the "two aspects" of communicative interference from the work of Baylor and colleagues (2011), it appears that comprehensive interventions for those with communication impairments should target both the internal and external sources of interference. If successful, these findings suggest that an effective intervention could increase social self-efficacy and, in turn, 
enhance social interactions and psychological well-being. Improvements in psychological outcomes have been demonstrated after a wide range of interventions for communication impairments (Baylor et al., 2007; Boi et al., 2012; Hawkins, 2005; Heydebrand et al., 2005; Liu et al., 1998; Mulrow, Aguilar, Endicott, Tuley, et al., 1990; Murry et al., 1994). What is less well-known, however, is whether aspects of social relationships are responsive to these types of interventions. Based on the findings from the current study it would appear that, in addition to loneliness and depression, the impact of interventions for a communication impairment on esteem support, belonging support, the contribution of friends to the social network, and social participation are targets for future investigations.

\section{Conclusions}

The findings from this study support an association between communication impairment and several important aspects of social relationships in older adults. Even after controlling for demographic characteristics, health, and disability, communication impairment was a significant independent predictor for fewer friends in the social network, a reduction in certain components of social support, and reduced social participation. Communication impairment did not significantly predict overall levels of social support but was significantly associated with lower levels of social integration (network support) and reassurance of worth (esteem support). Communication impairment also significantly predicted higher levels of loneliness and depression, and reduced social self-efficacy. With regard to psychological well-being, no interaction between communication impairment and social support was found nor between 
communication impairment and physical disability. Evidence for two distinct pathways was found between communication impairment and psychological well-being. Communication was a significant predictor of loneliness after controlling for the mediator, social self-efficacy, consistent with partial mediation. For depression, however, communication was no longer a significant predictor after controlling for the mediators social self-efficacy and reassurance of worth, consistent with full mediation. Taken together these two characteristics, namely social self-efficacy and reassurance of worth, appeared to be important in explaining the connection between communication and psychological well-being. 


\section{References}

Adams, R.G., \& Blieszner, R. (1995). Aging well with friends and family. American Behavioral Scientist, 39, 209-224.

Adams, K.B., Sanders, S., \& Auth, E.A. (2004). Loneliness and depression in independent living retirement communities. Aging \& Mental Health, 8, 475-485. Adelman, R.D., Greene, M.G., \& Ory, M.G. (2000). Communication between older patients and their physicians. Clinics in Geriatric Medicine, 16, 1-24.

Aldwin, C.M., \& Gilmer, D.F. (2004). Health, illness and optimal aging: Biological and psychological perspectives. Thousand Oaks, CA: Sage Publications, Inc.

Allan, G. (1989). Friendships: Developing a sociological perspective. London: Allen \& Unwin.

Almeida, D.M., Piazza, J.R., Stawski, R.S., \& Klein, L.C. (2011). The speedometer of life: Stress, health and aging. In K.W. Schaie and S.L. Willis, S.L. (Eds.), Handbook of the psychology of aging (7th Ed., pp.191-206). Burlington, MA: Academic Press.

American Speech-Language-Hearing Association. (1988). Determining threshold level for speech. Rockville, MD: ASHA. Retrieved from www.asha.org/policy.

American Speech-Language-Hearing Association. (1997). Guidelines for audiologic screening. Rockville, MD: ASHA. Retrieved from www.asha.org/policy.

American Speech-Language-Hearing Association. (2005). Guidelines for manual puretone threshold audiometry. Rockville, MD: ASHA. Retrieved from www.asha.org/policy.

American Speech-Language-Hearing Association. (2008). Incidence and prevalence of speech, voice, and language disorders in adults in the United States: 2008 edition. Rockville, MD: ASHA. Retrieved from www.asha.org/research.

Anderson, D. L., \& Noble, W. (2005). Couples' attributions about behaviours modulated by hearing impairment: Links with relationship satisfaction. International Journal of Audiology, 44, 197-205.

Andrews, G., Tennant, C., Hewson, D., \& Vaillant, G. (1978). Life stress, social support, coping style and risk of psychological impairment. Journal of Nervous \& Mental Disease, 166, 307-316.

Antonucci, T. (1990). Social supports and social relationships. In R. H. Binstock \& L. K. George (Eds.), Handbook of aging and the social sciences (3rd ed., pp. 205-226). New York: Academic Press. 
Antonucci, T.C. (2001). Social relations: An examination of social networks, social support, and sense of control. In J.E. Birren (Ed.), Handbook of the psychology of aging (5th ed., pp.427-453). San Diego, CA: Academic Press.

Antonucci, T.C., \& Akiyama, H. (1987). Social networks in adult life and a preliminary examination of the Convoy Model. Journal of Gerontology, 42, 519-527.

Antonucci, T.C., \& Jackson, J.S. (1987). Social support, interpersonal self-efficacy, and health: A life-course perspective. In L.L. Carstensen \& B.A. Edelstein (Eds.), Handbook of clinical gerontology (pp. 291-311). Elmsford, NY: Pergamon Press.

Anyanwu, U. O., Sharkey, J. R., Jackson, R. T., \& Sahyoun, N. R. (2011). Home food environment of older adults transitioning from hospital to home. Journal of nutrition in gerontology and geriatrics, 30, 105-121.

Archbold, P.B., Stewart, B.J., Greenlick, M.R., \& Harvath, T. (1990). Mutuality and preparedness as predictors of caregiver role strain. Research in Nursing and Health, 13, 375-84.

Arlinger, S. (2003). Negative consequences of uncorrected hearing loss: A review. International Journal of Audiology, 42(2), S17-S20.

Babbitt, E.M., \& Cherney, L.R. (2010). Communication confidence in persons with aphasia. Topics in Stroke Rehabilitation, 17, 214-223.

Baikie, E. (2002). The impact of dementia on marital relationships. Sexual and Relationship Therapy, 17, 289-99. doi: 10.1080/14681990220149095

Balandin, S., Berg, N., \& Waller, A. (2006). Assessing the loneliness of older people with cerebral palsy. Disability \& Rehabilitation, 28, 469-479.

Ball, L.J., Beukelman, D.R., Pattee, G.L. (2004). Communication effectiveness of individuals with amyotrophic lateral sclerosis. Journal of Communication Disorders, 37, 197-215.

Ballin, L. \& Balandin, S. (2007). An exploration of loneliness: Communication and the social networks of older people with cerebral palsy. Journal of Intellectual and Developmental Disability, 32, 315-327.

Baltes, M.M. (1996). The many faces of dependency in old age. New York: Cambridge University Press.

Baltes, M.M., \& Carstensen, L.L. (1996). The process of successful aging. Ageing and Society, 16, 397-422. 
Baltes, M.M., \& Carstensen, L.L. (1999). Social-psychological theories and their applications to aging: From individual to collective. In V.L. Bengtson \& K.W. Schaie (Eds.), Handbook of theories of aging (pp. 209-226). New York: Springer Publishing Co.

Baltes, P.B. (1987). Theoretical propositions of life-span developmental psychology: On the dynamics between growth and decline. Developmental Psychology, 23, 611626.

Baltes, P.B. (1997). On the incomplete architecture of human ontogeny: Selection, optimization, and compensation as foundation of developmental theory. American Psychologist, 52, 366-380.

Baltes, P.B., \& Baltes, M.M. (Eds.) (1990). Successful aging: Perspectives from the behavioral sciences. New York: Cambridge University Press.

Bandura, A. (1977). Self-efficacy: Towards a unifying theory of behavior change. Psychological Review, 84, 191-215.

Bandura, A. (1986). Social foundations of thought and action: A social cognitive theory. Englewood Cliffs, NJ: Prentice-Hall.

Bandura, A. (1997). Self efficacy: The exercise of control. New York: Freeman.

Barrera, M. (1986). Distinctions between social support concepts, measures, and models. American Journal of Community Psychology, 14, 413-445.

Barrera, M. (2000). Social support research in community psychology. In J. Rappaport \& E. Seidman (Eds.), Handbook of community psychology (pp. 215-245). New York: Kluwer Academic/Plenum.

Bassuk, S.S., Glass, T.A., \& Berkman, L.F. (1999). Social disengagement and incident cognitive decline in community-dwelling elderly persons. Annals of Internal Medicine, 131, 165-173.

Baxter, L.A., Braithwaite, D.O., Golish, T.D., \& Olson, L.N. (2002). Contradictions of interaction for wives of elderly husbands with adult dementia. Journal of Applied Communication Research, 30, 1-26.

Baylor, C., Burns, M., Eadie, T., Britton, D., \& Yorkston, K. (2011). A qualitative study of interference with communicative participation across communication disorders in adults. American Journal of Speech-Language Pathology, 20, 269-287.

Baylor, C., Yorkston, K., Bamer, A., Britton, D., \& Amtmann, D. (2010). Variables associated with communicative participation in people with multiple sclerosis: A 
regression analysis. American Journal of Speech-Language Pathology, 19, 143153.

Baylor, C.R., Yorkston, K.M., Eadie, T.L., \& Maronian, N.C. (2007). The psychosocial consequences of BOTOX injections for spasmodic dysphonia: A qualitative study of patients' experiences. Journal of Voice, 21, 231-47.

Berg, E.E, Hapner, E., Klein, A., Johns, M.M. (2008). Voice therapy improves quality of life in age-related dysphonia: A case-control study. Journal of Voice. 22, 70-74.

Berg, S., Mellström, D., Persson, G., \& Svanborg, A. (1981). Loneliness in the Swedish aged. Journal of Gerontology, 36, 342-349.

Berkman, L.F. (1995). The role of social relations in health promotion. Psychosomatic Medicine, 57, 245-254.

Berkman, L.F., \& Glass, T. (2000). Social integration, social networks, social support, and health. In L.F. Berkman \& I. Kawachi (Eds.), Social epidemiology (pp. 137173). Oxford, UK: Oxford University Press.

Berkman, L.F., Glass, T., Brissette, I., \& Seeman, T.E. (2000). From social integration to health: Durkheim in the new millennium. Social Science \& Medicine, 51, 843857.

Berkman, L.F., \& Syme, L. (1979). Social networks, host resistance, and mortality: A nine-year follow-up study of Alameda County residents. American Journal of Epidemiology, 109, 186-204.

Berry, P., Mascia, J., \& Steinman, B.A. (2004). Vision and hearing loss in older adults: "Double trouble." Care Management Journals, 5, 35-40. doi: 10.1891/cmaj.5.1.35.61260

Bisconti, T.L., \& Bergeman, C.S. (1999). Perceived social control as a mediator of the relationships among social support, psychological well-being, and perceived health. The Gerontologist, 39, 94-103.

Blau, P. (1964). Exchange and power in social life. New York: Wiley.

Blay, S.L., Andreoli, S.B., Fillenbaum, G.G., \& Gastal, F.L. (2007). Depression morbidity in later life: Prevalence and correlates in a developing country. American Journal of Geriatric Psychiatry, 15, 790-9.

Blood, G.W., Luther, A.R., \& Stemple, J.C. (1992). Coping and adjustment in alaryngeal speakers. American Journal of Speech-Language Pathology, 1, 63-69. 
Blood, G.W., Simpson, K.C., Raimondi, R.C., Dineen, M., Kauffman, S.M., \& Stagaard, K.A. (1994). Social support in laryngeal cancer survivors: Voice and adjustment issues. American Journal of Speech-Language Pathology, 3, 37-44.

Boerner, K., \& Wang, S.-W. (2010). How it matters when it happens: Life changes related to functional loss in younger and older adults. International Journal of Aging and Human Development, 70, 163-179.

Boi, R., Racca, L., Cavallero, A., Carpaneto, V., Racca, M., Dall'Acqua, F., ... \& Odetti, P. (2012). Hearing loss and depressive symptoms in elderly patients. Geriatrics \& Gerontology International, 12, 440-445..

Bolger, N., DeLongis, A., Kessler, R.C., \& Schilling, E.A. (1989). Effects of daily stress on negative mood. Journal of Personality and Social Psychology, 57, 808-818.

Bombardier, C.H., Ehde, D.M., Stoelb, B., \& Molton, I.R. (2010). The relationship of age-related factors to psychological functioning among people with disabilities. Physical Medicine \& Rehabilitation Clinics of North America, 21, 281-297.

Bonilha, H.S. \& Dawson, A.E. (2012). Creating a mastery experience during the voice evaluation. Journal of Voice, 26, 665, e1-7.

Bourque, P., Leger, C., Pushkar, D., \& Beland, F. (2007). Self-reported sensory impairment and life satisfaction in older French-speaking adults. Canadian Journal of Nursing Research, 39, 155-71.

Bowling, A. (1994). Social networks and social support among older people and implications for emotional well-being and psychiatric morbidity. International Review of Psychiatry, 6, 41-58.

Brainerd, S.H., \& Frankel, B.G. (1985). The relationship between audiometric and selfreport measures of hearing handicap. Ear and Hearing, 6, 89-92.

Brennan, M., Horowitz, A., \& Su, Y. (2005). Dual sensory loss and its impact on everyday competence. The Gerontologist, 45, 337-346. doi: 10.1093/geront/45.3.337

Brennan, M., Su, Y., \& Horowitz, A., (2006). Longitudinal associations between dual sensory impairment and everyday competence among older adults. Journal of Rehabilitation Research \& Development, 43, 777-792. doi: 10.1682/JRRD.2005.06.0109

Bricker-Katz, G., Lincoln, M., \& McCabe, P. (2009). A life-time of stuttering: How emotional reactions to stuttering impact activities and participation in older people. Disability and Rehabilitation, 31, 1742-1752. 
Bricker-Katz, G., Lincoln, M., \& McCabe, P. (2010). Older people who stutter: Barriers to communication and perceptions of treatment needs. International Journal of Language \& Communication Disorders, 45, 15-30.

Bringfelt, P.-A., Hartelius, L., \& Runmarker, B. (2006). Communication problems in multiple sclerosis: 9-year follow-up. International Journal of Multiple Sclerosis Care, 8, 130-140.

Brooks, D.N., Hallam, R.S., \& Mellor, P.A. (2001). The effects on significant others of providing a hearing aid to the hearing-impaired partner. British Journal of Audiology, 35, 165-71.

Bulmer, M. (1987). The social basis of community care. Boston, MA: Allen \& Unwin.

Burns, C.L. \& Farina, A. (1984). Social competence and adjustment. Journal of Social \& Personal Relationships, 1, 99-113.

Bute, J.J., Donovan-Kicken, E., \& Martins, N. (2007). Effects of communicationdebilitating illnesses and injuries on close relationships: A relational maintenance perspective. Health Communication, 21, 235-246.

Cacioppo, J.T., \& Hawkley, L.C. (2003). Social isolation and health, with an emphasis on underlying mechanisms. Perspectives in Biology \& Medicine, 46, S39-S52.

Calman, K. (1987). Definitions and dimensions of quality of life. In N. K. Aaronson \& J. Beckmann (Eds.), The quality of life of the cancer patient (pp. 1-9). New York. NY: Raven Press.

Cantor, M. (1979). Neighbors and friends: An overlooked resource in the informal support system. Research on Aging, 1, 434-463.

Cantor, M. (1980). The informal support system: Its relevance in the lives of the elderly. In E. Borgatta and N. McCluskey (Eds.), Aging and Society. Beverly Hills, CA: Sage Publications.

Capella-McDonnall, M.E. (2005). Effects of single and dual sensory loss on symptoms of depression in the elderly. International Journal of Geriatric Psychiatry, 20, 855861 .

Carozza, L., \& Shafi, N. (2013). Quality of life in aphasia community group members: A social model of clinical treatment. Acta Neuropsychologica, 11, 1-7.

Carstensen, L.L. (1991). Selectivity theory: Social activity in a life-span context. Annual Review of Gerontology \& Geriatrics, 11, 195-217. 
Carstensen, L.L., Isaacowitz, D.M., \& Charles, S.T. (1999). Taking time seriously: A theory of socioemotional selectivity. American Psychologist, 54, 165-181.

Carter, J. H., Stewart, B. J., Archbold, P. G., Inoue, I., Jaglin, J., Lannon, M., ... \& Zoog, K. (1998). Living with a person who has Parkinson's disease: The spouse's perspective by stage of disease. Movement Disorders, 13, 20-28.

Centers for Disease Control and Prevention (2013). International classification of diseases, ninth revision, clinical modification (ICD-9-CM). Atlanta, GA: CDC. Available from: http://www.cdc.gov/nchs/icd/icd9cm.htm

Chalres, S.T. (2011). Emotional experience and regulation in later life. In K.W. Schaie and S.L. Willis, S.L. (Eds.), Handbook of the psychology of aging (7th Ed., pp.295-310). Burlington, MA: Academic Press.

Charness, N. (2000). Can acquired knowledge compensate for age-related declines in cognitive efficiency? In S.H. Qualls \& N. Abeles (Eds.), Psychology and the aging revolution: How we adapt to longer life (pp. 99-117). Washington, DC: American Psychological Association.

Chatters, L.M., Taylor, R.J., \& Jackson, J.S. (1985). Size and composition of the informal helper networks of elderly Blacks. Journal of Gerontology, 40, 605-614.

Chen, H.L. (1994). Hearing in the elderly: Relation of hearing loss, loneliness, and selfesteem. Journal of Gerontological Nursing, 20, 22-28.

Chou, K.-L. (2008). Combined effect of vision and hearing impairment on depression in older adults: Evidence from the English Longitudinal Study of Ageing. Journal of Affective Disorders, 106, 191-196.

Chou, K.L., \& Chi, I. (1999). Determinants of life satisfaction in Hong Kong Chinese elderly: A longitudinal study. Aging and Mental Health, 3, 328-335.

Christakis, N., \& Fowler, J. (2009). Connected: The amazing power of social networks and how they shape our lives. New York: Little, Brown and Co.

Clark, J.H., Yeagle, J., Arbaje, A.I., Lin, F.R., Niparko, J.K., \& Francis, H.W. (2012). Cochlear implant rehabilitation in older adults: Literature review and proposal of a conceptual framework. Journal of the American Geriatrics Society, 60, 19361945.

Cobb, S. (1979). Social support and health through the life course. In M. W. Riley (Ed.), Aging from birth to death: Interdisciplinary perspectives (pp. 93-106). Boulder, CO: Westview Press. 
Cohen, G.D. (2000). Loneliness in later life. American Journal of Geriatric Psychiatry, 8, 273-275.

Cohen, J. (1988). Statistical power analysis for the behavioral sciences. Hillsdale, New Jersey: Lawrence Erlbaum Associates.

Cohen, S. (1988). Psychosocial models of the role of social support in the etiology of physical disease. Health Psychology, 7, 269-297.

Cohen, S. (2004). Social relationships and health. American Psychologist, 59, 676-684.

Cohen, J., Cohen, P., West, S.G., \& Aiken, L.S. (2003). Applied multiple regression/correlation analysis for the behavioral sciences (3rd ed.). Mahwah, NJ: Erlbaum.

Cohen, S., \& Wills, T.A. (1985). Stress, social support, and the buffering hypothesis. Pyschological Bulletin, 98, 310-357.

Cohen, S., Underwood, L.G., \& Gottlieb, B.H. (2000). Social support measurement and intervention: A guide for health and social scientists. Oxford, UK: Oxford University Press.

Coleman, (1990). The foundations of social theory. Cambridge, MA: Harvard University Press.

Connidis, I.A. (2010). Family ties and aging. Thousand Oaks, CA: Pine Forge Press.

Connor-Smith, J.K., \& Flachsbart, C. (2007). Relations between personality and coping: A meta-analysis. Journal of Personality and Social Psychology, 93, 1080-1107.

Cornwell, B. (2011). Independence through social networks: Bridging potential among older women and men. The Journals of Gerontology, Series B: Psychological Sciences and Social Sciences, 66, 782-794.

Cresswell, J.W. (2007). Qualitative inquiry \& research design: Choosing among five approaches. Thousand Oaks, CA: Sage Publications.

Cresswell, J.W. (2013). Research design: Qualitative, quantitative, and mixed methods approaches. Thousand Oaks, CA: Sage Publications.

Cresswell, J.W., Plano Clark, V.L., Gutmann, M.L., \& Hanson, W.E. (2003). Advanced mixed methods research designs. In A. Tashakkori \& C. Teddlie (Eds.), Handbook of mixed methods in social \& behavioral research (pp. 209-240). Thousand Oaks, CA: Sage Publications. 
Crohan, S.E., \& Antonucci, T.C. (1989). Friends as a source of social support in old age. In R.G. Adams \& R. Blieszner (Eds.), Older adult friendship (pp. 129-146). Newbury Park, CA: Sage.

Cruice, M. (2002). Communication and quality of life in older people with aphasia and healthy older people. Unpublished doctoral thesis. The University of Queensland, Brisbane, Australia. Retrieved from http://espace.library.uq.edu.au/view/UQ:106028

Cruice, M., Worrall, L., \& Hickson, L. (2000). Quality-of-life measurement in speech pathology and audiology. Asia Pacific Journal of Speech, Language, and Hearing, 5, 1-20.

Cruice, M., Worrall, L., \& Hickson, L. (2005). Personal factors, communication and vision predict social participation in older adults. Advances in Speech-Language Pathology, 7, 220-232.

Cumming, E., \& Henry, W.E. (1961). Growing old: The process of disengagement. New York: Basic Books.

Curran, P.J., West, S.G., \& Finch, J. (1996). The robustness of test statistics to nonnormality and specification error in confirmatory factor analysis. Psychological Methods, 1, 16-29.

Cutrona, C.E. (1986). Objective determinants of perceived social support. Journal of Personality and Social Psychology, 50, 349-55.

Cutrona, C.E., \& Russell, D.W. (1987). The provisions of social relationships and adaptation to stress. Advances in Personal Relationships, 1, 37-67.

Cutrona, C.E., Russell, D.W., \& Rose, J. (1986). Social support and adaptation to stress by the elderly. Journal of Psychology and Aging, 1, 47-54.Cutrona, C.E., \& Russell, D.W. (1990). Type of social support and specific stress: Towards a theory of optimal matching. In B.R. Sarason, I.G. Sarason, \& G.R. Pierce (Eds.), Social support: An interactional view (pp. 319-366). New York: Wiley.

Dalemans, R., de Witte, L.P., Lemmens, J., van den Heuvel, W.J.A., \& Wade, D.T. (2008). Measures for rating social participation in people with aphasia: A review. Clinical Rehabilitation, 22, 542-555.

Dalton, D.S., Cruickshanks, K.J., Klein, B.E., Klein, R., Wiley, T.L., \& Nondahl, D.M. (2003). The impact of hearing loss on quality of life in older adults. The Gerontologist, 43, 661-8. 
Davidson, B., Howe, T., Worrall, L., Hickson, L., \& Togher, L. (2008). Social participation for older people with aphasia: The impact of communication disability on friendships. Topics in Stroke Rehabilitation, 15, 325-340.

Davidson, B., Worrall, L., \& Hickson, L. (1998, August). Observed communication activities of people with aphasia and healthy older people. In 8th International Aphasia Rehabilitation Conference, Kwa Maritane, South Africa.

de Graaf, R., \& Bijl, R.V. (2002). Determinants of mental distress in adults with a severe auditory impairment: Differences between prelingual and postlingual deafness. Psychosomatic Medicine, 64, 61-70.

de Jong Gierveld, J. \& Havens, B. (2004). Cross-national comparisons of social isolation and loneliness: Introduction and overview. Canadian Journal on Aging, 23, 109113.

Deary, I.J., Wilson, J.A., Carding, P.N., \& Mackenzie, K. (2003). The dysphonic voice heard by me, you and it: Differential associations with personality and psychological distress. Clinical Otolaryngology \& Allied Sciences, 28, 374-8. doi: 10.1046/j.1365-2273.2003.00730.x

DeVellis, R. (2003). Scale development: Theory and applications. Thousand Oaks, CA: SAGE.

Dickens, A.P., Richards, S.H., Greaves, C.J., \& Campbell, J.L. (2011). Interventions targeting social isolation in older adults: A systematic review. BMC Public Health, 11, 647.

Dickens, W.J., \& Perlman, D. (1981). Friendship over the lifecycle. In S. Duck \& R. Gilmour (Eds.), Personal relationships 2: Developing personal relationships. London: Academic Press.

Dijkers, M.P.J.M., Whiteneck, G., \& El-Jaroudi, R. (2000). Measures of social outcomes in disability research. Archives of Physical Medicine and Rehabilitation, 81, S63S80.

Dixon, R.A., \& Backman, L. (1995). Psychological compensation: Managing losses and promoting gains (pp. 35-79). Hillsdale, NJ: Erlbaum.

Dono, J.E., Falbe, C.M., Kail, B.L., Litwak, E., Sherman, R.H., \& Siegel, D. (1979). Primary groups in old age: Structure and function. Research on Aging, 1, 403433.

Donovan, N.J., Kendall, D.L., Young, M.E., \& Rosenbek, J.C. (2008). The Communicative Effectiveness Survey: Preliminary evidence of construct validity. American Journal of Speech-Language Pathology, 17, 335-347. 
Dowd, J. J. (1975). Aging as exchange: A preface to theory. Journal of Gerontology, 30, 584-594.

Dugan, E., \& Kivett, V.R. (1994). The importance of emotional and social isolation to loneliness among very old rural adults. The Gerontologist, 34, 340-346.

Dunkel-Schetter, C., \& Bennett, T.L. (1990). Differentiating the cognitive and behavioral aspects of social support. In B.R. Sarason, I.G. Sarason, \& G.R. Pierce (Eds.), Social support: An interactional view (pp. 267-296). New York: Wiley.

Durkheim, E. (1897, 1951). Suicide: A study in sociology. Glencoe, IL: Free Press.

Eadie, T.L. (2003). The ICF: A proposed framework for comprehensive rehabilitation of individuals who use alaryngeal speech. American Journal of Speech-Language Pathology, 12, 189-197.

Eadie, T. L., \& Bowker, B. C. (2012). Coping and quality of life after total laryngectomy. Otolaryngology-Head and Neck Surgery, 146, 959-965.

Ebert, D.A., \& Heckerling, P.S. (1998). Communication disabilities among medical inpatients. New England Journal of Medicine, 339, 272-3.

Eckenrode, J. (1983). The mobilization of social supports: Some individual constraints. American Journal of Community Psychology, 11, 509-528.

Edelbrock, D., Buys, L., Creasey, H., \& Broe, G.A. (2001). Social support, social networks and social isolation: The Sydney older persons' study. Australasian Journal of Ageing, 20, 173-178.

Eisenberger, N. I., Lieberman, M. D., \& Williams, K. D. (2003). Does rejection hurt? An fMRI study of social exclusion. Science, 302 (5643), 290-292.

Eizenmann, D.R., Nesselroade, J.R., Featherman, D.L., \& Rowe, J.W. (1997). Intraindividual variability in perceived control in an older sample: The MacArthur successful aging studies. Psychology and Aging, 12, 489-502.

Enderby, P., \& John, A. (1997). Therapy outcome measures: Speech-language pathology. Clifton Park, NY: Delmar Learning.

Engström, K., Mattsson, F., Järleborg, A., \& Hallqvist, J. (2008). Contextual social capital as a risk factor for poor self-rated health: A multilevel analysis. Social Science \& Medicine, 66, 2268-2280.

Eriksson, M. Social capital and health: Implications for health promotion. Global Health Action, 4, 5611. doi: 10.3402/gha.v4i0.5611 
Farley, A., McLafferty, E., \& Hendry, C. (2006). The physiological effects of ageing on the activities of living. Nursing Standard, 20 (45), 46-52.

Felton, B.J., \& Berry, C.A. (1992). Do the sources of the urban elderly's social support determine its psychological consequences? Psychology and Aging, 7, 89-97.

Finch, J.F., Okun, M.A., Pool, G.J., \& Ruehlman, L.S. (1999). A comparison of the influence of conflictual and supportive interactions on psychogical distress. Journal of Personality, 67, 581-621.

Finn, P. (2003). Addressing generalization and maintenance of stuttering treatment in the schools: A critical look. Journal of Communication Disorders, 36, 153-64.

Fiori, K.L., Antonucci, T.C., \& Cortina, K.S. (2006). Social network typologies and mental health among older adults. Journal of Gerontology: Psychological Sciences, 61B, P25-P32.

Fiori, K.L., McIlvane, J.M., Brown, E.E., \& Antonucci, T.C. (2006). Social relations and depressive symptomatology: Self-efficacy as a mediator. Aging \& Mental Health, 10, 227-239.

Fletcher, B.S., Cohen, M.Z., Schumacher, K., \& Lydiatt, W. (2012). A blessing and a curse: Head and neck cancer survivors' experiences. Cancer Nursing, 35, 126-32.

Folkman, S., Lazarus, R.S., Dunkel-Schetter, C., DeLongis, A., \& Gruen, R.J. (1986). Dynamics of a stressful encounter: Cognitive appraisal, coping and encounter outcomes. Journal of Personality and Social Psychology, 50, 992-1003.

Folkman, S., \& Moskowitz, J.T. (2004). Coping: Pitfalls and promise. Annual Review of Psychology, 55, 745-774.

Frankel, B.G., \& Turner, R.J. (1983). Psychological adjustment in chronic disability: The role of social support in the case of the hearing impaired. The Canadian Journal of Sociology / Cahiers canadiens de sociologie, 8, 273-291. doi: 10.2307/3340106

Frederickson, B.L., \& Carstensen, L.L. (1990). Choosing social partners: How old age and anticipated endings make us more selective. Psychology \& Aging, 5, 335-347.

Fung, H.H., Carstensen, L.L., \& Lutz, A.M. (1999). Influence of time on social preferences: Implications for life-span development. Psychology \& Aging, 14, 595-604.

George, L. (1989). Stress, social support, and depression over the life-course. In K. S. Markides \& C. L. Cooper (Eds.), Aging, stress, and health (pp. 241-267). New York: Wiley. 
Gillespie, A.I. \& Abbott, K.V. (2011). The influence of clinical terminology on selfefficacy for voice. Logopedics, Phoniatrics, Vocology, 36, 91-9.

Golant, S.M. (2003). Conceptualizing time and behavior in environmental gerontology. The Gerontologist, 43, 638-648.

Gomez, R.G., \& Madey, S.F. (2001). Coping-with-hearing-loss model for older adults. Journal of Gerontology-Psychological Sciences, 56B, P223-P225.

Granovetter, M.S. (1973). The strength of weak ties. American Journal of Sociology, 78, 1360-1380.

Grenade, L. \& Boldy, D. (2008). Social isolation and loneliness among older people: Issues and future challenges in community and residential settings. Australian Health Review, 32, 468-478.

Giles, H., Coupland, N., \& Coupland, J. (Eds.) (1991). The contexts of accommodation. New York: Cambridge University Press.

Greene, J.C., \& Caracelli, V.J. (1997). Defining and describing the paradigm issue in mixed-method evaluation. In J.C. Greene \& V.J. Caracelli (Eds.), Advances in mixed method evaluation: The challenges and benefits of integrating diverse paradigms (New Directions for Evaluation, No. 74, pp. 5-17). San Francisco, CA: Jossey-Bass.

Hall, A., \& Wellman, B. (1985). Social networks and social support. In S. Cohen \& S.L. Syme (Eds.), Social support and health, (pp. 23-41). Orlando, FL: Academic Press.

Halpern, A. E., Ramig, L. O., Matos, C. E., Petska-Cable, J. A., Spielman, J. L., Pogoda, J. M., ... \& McFarland, D. H. (2012). Innovative technology for the assisted delivery of intensive voice treatment (LSVT LOUD) for Parkinson Disease. American Journal of Speech-Language Pathology, 21, 354.

Harada, S., Nishiwaki, Y., Michikawa, T., Kikuchi, Y., Iwasawa, S., Nakano, M., ... \& Takebayashi, T. (2008). Gender difference in the relationships between vision and hearing impairments and negative well-being. Preventive Medicine, 47, 433-437. doi: 10.1016/j.ypmed.2008.06.011

Havighurst, R.J., \& Albrecht, R. (1953). Older people. New York: Longmans.

Hawkins, D.B. (2005). Effectiveness of counseling-based adult group aural rehabilitation programs: A systematic review of the evidence. Journal of the American Academy of Audiology, 16, 485-493. doi: 10.3766/jaaa.16.7.8 
Hawthorne, G. (2008). Perceived social isolation in a community sample: Its prevalence and correlates with aspects of peoples' lives. Social Psychiatry and Psychiatric Epidemiology, 43, $140-150$.

Heine, C. \& Browning, C.J. (2002). Communication and psychosocial consequences of sensory loss in older adults: Overview and rehabilitation directions. Disability \& Rehabilitation, 24, 763-773

Heine, C., Erber, N.P., Osborn, R., \& Browning, C.J. (2002). Communication perceptions of older adults with sensory loss and their communication partners: Implications for intervention. Disability \& Rehabilitation, 24, 356-363. doi: $10.1080 / 09638280110096250$

Henretta, J. C., Hill, M. S., Li, W., Soldo, B. J., \& Wolf, D. A. (1997). Selection of children to provide care: The effect of earlier parental transfers. Journals of Gerontology: Psychological and Social Sciences, 52B (Special issue), 110-119.

Herlitz, J., Wiklund, I., Caidahl, K., Hartford, M., Haglid, M., Karlsson, B. W., ... \& Karlsson, T. (1998). The feeling of loneliness prior to coronary artery bypass grafting might be a predictor of short-and long-term postoperative mortality. European Journal of Vascular and Endovascular Surgery, 16, 120-125.

Hétu, R., Jones, L., \& Getty, L., (1993). The impact of acquired hearing impairment on intimate relationships: Implications for rehabilitation. Audiology, 32, 363-381.

Heydebrand, G., Mauze, E., Tye-Murray, N., Binzer, S., \& Skinner, M. (2005). The efficacy of a structured group therapy intervention in improving communication and coping skills for adult cochlear implant recipients. International Journal of Audiology, 44, 272-80. doi: 10.1080/14992020500060404

Hickson, L.M.H., Worrall, L.E., Barnett, H.M., \& Yiu, E.M.-L. (1995). The relationship between communication skills, social networks and decision-making strategies: An exploratory study. Australian Journal on Aging, 14, 89-94.

Hilari, K., \& Northcott, S. (2006). Social support in people with chronic aphasia. Aphasiology, 20, 17-36.

Hilari, K., Northcott, S., Roy, P., Marshall, J., Wiggins, R. D., Chataway, J., \& Ames, D. (2010). Psychological distress after stroke and aphasia: The first six months. Clinical Rehabilitation, 24, 181-190.

Hoenig, H., Nusbaum, N., Brummel-Smith, K. (1997). Geriatric rehabilitation: State of the art. Journal of the American Geriatrics Society, 45, 1371-81.

Hoffman, J.M., Yorkston, K.M., Shumway-Cook, A., Ciol, M.A., Dudgeon, B.J., \& Chan, L. (2005). Effect of communication disability on satisfaction with health 
care: A survey of Medicare beneficiaries. American Journal of Speech-Language Pathology, 14, 221-228.

Holmes, D., \& Rahe, R. (1967). The Social Readjustment Rating Scale. Journal of Psychosomatic Research, 11, 213-218.

Holt-Lunstad, J., Smith, T.B., \& Layton, J.B. (2010). Social relationships and mortality risk: A meta-analytic review. PLos Med 7(7): e1000316. Retrieved from: www.plosmedicine.org

Homans, C.G. (1958). Social behavior as exchange. American Journal of Sociology, 62, 597-606.

Horgas, A.L., Wilms, H.-U., \& Baltes, M.M. (1998). Daily life in old age: Everyday activities as expression of successful aging. The Gerontologist, 38, 556-568.

Horowitz, A, Brennan, M., \& Su, Y.-P. (2001). Dual sensory impairment among the elderly. New York, NY: Arlene R. Gordon Research Institute, Lighthouse International. Retrieved from: http://www.lighthouse.org/research/archivedstudies/dual/

House, J.S. (2001). Social isolation kills, but how and why? Psychosomatic Medicine, 63, 273-274.

House, J.S., Landis, K.R., \& Umberson, D. (1988). Social relationships and health. Science, 241, 540-545.

IBM Corp. (2010). IBM SPSS Statistics for Windows, Version 19.0. Armonk, NY: IBM Corp.

Islam, M. K., Merlo, J., Kawachi, I., Lindström, M., \& Gerdtham, U. G. (2006). Social capital and health: Does egalitarianism matter? A literature review. International Journal for Equity in Health, 5, 3.

Iwarsson, S. (2005). A long-term perspective on person-environment fit and ADL dependence among older Swedish adults. The Gerontologist, 45, 327-336.

Jacobson, B.H., Johnson, A., Grywalski, C., Silbergleit, A., Jacobson, G., Benninger, M.S., \& Newman, C.W. (1997). The voice handicap index (VHI): Development and validation. American Journal of Speech-Language Pathology, 6, 66-70.

Johnson, C.L., \& Catalano, D.J. (1983). A longitudinal study of family support to impaired elderly. The Gerontologist, 23, 612-618. 
Joint Commission, The (2010). Advancing effective communication, cultural competence, and patient- and family-centered care: A roadmap for hospitals. Oakbrook Terrace, IL: The Joint Commission.

Jones, E.M., \& White, A.J. (1990). Mental health and acquired hearing impairment: A review. British Journal of Audiology, 24, 3-9. doi: 10.3109/03005369009077837

Joubert, K., Bornman, J., \& Alant, E. (2011). Speech intelligibility and marital communication in Amyotrophic Lateral Sclerosis: An exploratory study. Communication Disorders Quarterly, 33, 34-41. doi: $10.1177 / 1525740110367824$.

Judge, S., Clarke, Z. and Hawley, M. S. (2011) Investigating the success factors of expert users to inform device development. In G. J. Gelderblom, , M. Soede, , L. Adriaens, \& K. Miesenberger (Eds.), Everyday Technology for Independence and Care - AAATE 2011 (pp. 995-1003). Maastricht, The Netherlands: IOS Press. doi: 10.3233/978-1-60750-814-4-995

Kahana, E. (1982). A congruence model of person-environment interaction. In M.P. Lawton, P. Windley, \& T. Byerts (Eds.), Aging and the environment: Theoretical approaches (pp. 97-121). New York: Springer Publishing Co.

Kahn, R.L. (1979). Aging and social support. In M. W. Riley (Ed.), Aging from birth to death: Interdisciplinary perspectives (pp. 77-91). Boulder, CO: Westview Press.

Kahn, R.L. \& Antonucci, T.C. (1980). Convoys over the lifecourse: Attachment, roles and social support. In P.B. Baltes \& O. Brim (Eds.), Life span development and behavior (pp. 253-286). New York: Academic Press.

Kahn, J. R., \& Pearlin, L. I. (2006). Financial strain over the life course and health among older adults. Journal of Health and Social Behavior, 47, 17-31.

Katz, S., Ford, A.B., Moskowitz, R.W., Jackson, B.A., \& Jaffe, M.W. (1963). Studies of illness in the aged. The index of ADL: A standardized measure of biological and psychosocial function. Journal of the American Medical Association, 185, 914919. doi: 10.1001/jama.1963.03060120024016

Kauhanen, M.L., Korpelainen, J.T., Hiltunen, P., Määttä, R., Mononen, H., Brusin, E., ... \& Myllylä, V.V. (2000). Aphasia, depression, and non-verbal cognitive impairment in ischaemic stroke. Cerebrovascular Diseases, 10, 455-61. doi: $10.1159 / 000016107$

Kaul, M., \& Lakey, B. (2003). Where is the support in perceived support? The role of generic relationship satisfaction and enacted support in perceived support's relation to low distress. Journal of Social and Clinical Psychology, 22, 59-78. 
Kawachi, I., Subramanian, S.V., \& Kim, D. Social capital and health: A decade of progress and beyond. In D. Kim (Ed.), Social capital and health, (pp. 1-X). New York: Springer.

Kawachi, I., Kennedy, B.P., Lochner, K., \& Prothrow-Stith, D. (1997). Social capital, income inequality, and mortality. American Journal of Public Health, 87, 14911498. doi: 10.2105/AJPH.87.9.1491

Kempen, G.I.J.M., Verbrugge, L.M., Merrill, S.S., \& Ormel, J. (1998). The impact of multiple impairments on disability in community-dwelling older people. Age and Ageing, 27, 595-604.

Kemper, S. (1994). Elderspeak: Speech accommodations to older adults. Aging and Cognition, 1, 17-28.

Kemper, S., Lyons, K., \& Anagnopoulos, C. (1995). Joint storytelling by patients with Alzheimer's disease and their spouses. Discourse Processes, 20, 205-217.

Kendig, H.L., Coles, R., Pittelkow, Y., \& Wilson, S. (1988). Confidants and family structure in old age. Journal of Gerontology, 43, S31-40.

Kiecolt-Glaser, J.K. (1999). Stress, personal relationships, and immune function: Health implications. Brain, Behavior, and Immunity, 13, 61-72.

Kim, D. (2008). Social capital and health. New York: Springer.

Kim, D., Subramanian, S.V., \& Kawachi, I. (2008). Social capital and physical health: A systematic review of the literature. In I. Kawachi, S.V. Subramanian, \& D. Kim (Eds.), Social capital and health (pp. 139-190). New York: Springer.

Kivett, V.R. (1979). Discriminators of loneliness among rural elderly: Implications for intervention. The Gerontologist, 19, 108-115.

Kochkin, S., \& Rogin, C.M. (2000). Quantifying the obvious: The impact of hearing instruments on quality of life. Hearing Review, 7, 6-34. Retrieved from: http://www.betterhearing.org/pdfs/Hearing_aids_and_quality_of_life_NCOA.pdf

Kramer, S.E., Kapteyn, T.S., Kuik, D., \& Deeg, D.J.H. (2002). The association of hearing impairment and chronic diseases with psychosocial health status in older age. Journal of Aging and Health, 14, 122-137.

Krause, N. (1995). Negative interaction and satisfaction with social support among older adults. Journal of Gerontology: Psychological Sciences, 50B, 59-73. 
Krause, N. (2006a). Exploring the stress-buffering effects of church-based and secular social support on self-rated health in late life. Journal of Gerontology, 61, S35S43.

Krause, N. (2006b). Social relationships in late life. In R.H. Binstock \& L.K. George (Eds.), Handbook of aging and the social sciences $\left(6^{\text {th }}\right.$ ed., pp. 181-200). Amsterdam: Elsevier.

Krause, N. \& Jay, G. (1991). Stress, social support, and negative interactions in later life. Research on Aging, 13, 333-363. doi: 10.1177/0164027591133004

Krause, N., \& Rook, K.S. (2003). Negative interaction in late life: Issues in the stability and generalizability of conflict across relationships. Journal of Gerontology: Psychological Sciences, 58B, P88-P99.

Krause, N. \& Shaw, B.A. (2002). Negative interaction and changes in functional disability during late life. Journal of Social and Personal Relationships, 19, 339359.

Kwakkel, G., Wagenaar, R.C., Kollen, B.J., \& Lankhorst, G.J. (1996). Predicting disability in stroke: A critical review of the literature. Age \& Ageing, 25, 479-489.

Lachman, M.E. (1986). Locus of control in aging research: A case for multidimensional and domain-specific assessment. Journal of Psychology and Aging, 1, 34-40.

Lachman, M.E., Neupert, S.D., \& Agrigoroaei, S. (2011). The relevance of control beliefs for health and aging. In K.W. Schaie \& S.L. Willis (Eds.), Handbook of the psychology of aging (pp. 175-190). Burlington, MA: Academic Press.

Lachman, M.E., Rosnick, C.B., \& Rocke, C. (2009). The rise and fall of control beliefs in adulthood: Cognitive and biopsychosocial antecedents and consequences of stability and change over nine years. In H.B. Bosworth \& C. Herzog (Eds.), Aging and cognition: Research methodologies and empirical advances (pp. 143-160). Washington, DC.

Lakey, B., \& Lutz, C. J. (1996). Social support and preventive and therapeutic interventions. In G. R. Pierce, B. R. Sarason, \& I. G. Sarason (Eds.), Handbook of social support and the family (pp. 435-465). New York: Plenum Press.

Lang, F.R., \& Carstensen, L.L. (1994). Close emotional relationships in late life: Further support for proactive aging in the social domain. Psychology and Aging, 9, $315-$ 324.

LaRocco, J.M., \& Jones, A.P. (1978). Co-worker and leader support as moderators of stress-strain relationships in work situations. Journal of Applied Psychology, 63, 624-634. 
Larson, R., Mannell, R., \& Zuzanek, J. (1986). Daily well-being of older adults with friends and family. Psychology and Aging, 1, 117-126.

Lawton, M.P. (1982). Competence, environmental press, and adaptationof older people. In M.P. Lawton, P. Windley, \& T. Byerts (Eds.), Aging and the environment: Theoretical approaches (pp. 33-59). New York: Springer Publishing Co.

Lawton, M.P. (1987). Contextual perspectives: Psychosocial influences. In L.W. Poon (Ed.), Handbook for clinical memory assessment of older adults. Washington, DC: American Psychological Association.

Lawton, M.P., \& Brody, E.M. (1969). Assessment of older people: Self-maintaining and instrumental activities of daily living. The Gerontologist, 9, 179-186. doi: 10.1093/geront/9.3_Part_1.179

Lee, G.R. (1979). Children and the elderly: Interaction and morale. Research on Aging, 1, 335-360.

Lefcourt, H.M. (1984). Research with the locus of control construct: Extensions and limitations (Vol. 3). Orlando, FL: Academic Press.

Levasseur, M., Desrosiers, J., \& St-Cyr Tribble, D. (2008). Subjective quality-of-life predictors for older adults with physical disabilities. American Journal of Physical Medicine and Rehabilitation, 87, 830-841.

Levasseur, M., Richard, L., Gauvin, L., \& Raymond, E. (2010). Inventory and analysis of definitions of social participation found in the aging literature: Proposed taxonomy of social activities. Social Science \& Medicine, 71, 2141-2149.

Levasseur, M., St-Cyr Tribble, D., \& Desrosiers, J. (2009). Meaning of quality of life for older adults: Importance of human functioning components. Archives of Gerontology \& Geriatrics, 49, e91-e100.

Lewin, K. (1938). The conceptual representation and measurement of psychological forces. Durham, NC: Duke University Press.

Lewin, K. (1951). Field theory in social science. New York: Harper.

Li, Y., \& Ferraro, K. (2005). Volunteering and depression in later life: Social benefit or selection process? Journal of Health and Social Behavior, 46, 68-84.

Lin, F.R., Niparko, J.K., \& Ferrucci, L. (2010). Hearing loss prevalence in the United States. Archives of Internal Medicine, 171, 1851-2. doi:

10.1001/archinternmed.2011.506 
Lin, N., Simeone, R.S., Ehsel, W.M., \& Kuo, W. (1979). Social support, stressful life events and illness: A model and an empirical test. Journal of Health and Social Behavior, 20, 108-119.

Lind, C., Hickson, L., Worrall, L., Lovie-Kitchin, J., Yiu, Ed., \& Barnett, H. (2003). Hearing and vision impairment and the social networks of older Australians. Australasian Journal on Ageing, 1, 20-25.

Litwak, E. (1985). Helping older people: The complementary roles of informal networks and formal systems. New York: Guildford Press.

Liu, C. Y., Yu, J. M., Wang, N. M., Chen, R. S., Chang, H. C., Li, H. Y., ... \& Lu, C. S. (1998). Emotional symptoms are secondary to the voice disorder in patients with spasmodic dysphonia. General Hospital Psychiatry, 20, 255-259. doi: 10.1016/S0163-8343(98)00022-X

Lockey, K., Jennings, M.B., \& Shaw, L. (2010). Exploring hearing aid use in older women through narratives. International Journal of Audiology, 49, 542-9.

Lomas, J., Pickard, L., Bester, S., Elbard, H., Finlayson, A., \& Zoghaib, C. (1989). The Communicative Effectiveness Index: Development and psychometric evaluation of a functional communication measure for adult aphasia. Journal of Speech and Hearing Disorders, 54, 113-124.

Luanaigh, C.O., \& Lawlor, B.A. (2008). Loneliness and the health of older people. International Journal of Geriatric Psychiatry, 23, 1213-1221.

Lubben, J.E. (1988). Assessing social networks among elderly populations. Family and Community Health, 11, 42-52.

Lubben, J., \& Gironda, M. (2004). Measuring social networks and assessing their benefits. In C. Phillipson, G. Allan, \& D. Morgan (Eds.), Social networks and social exclusion (pp.20-34). Aldershot, England: Ashgate Publishing Co.

Lubben, J., Gironda, M., \& Lee, A. (2002). Refinements to the Lubben Social Network Scale: The LSNS-R. The Behavioral Measurement Letter, 7, 2-11.

Lubinski, R., \& Welland, R. J. (1997). Normal aging and environmental effects on communication. Seminars in Speech and Language, 18 (2), 107-126.

Magilvy, J.K. (1985). Quality of life of hearing-impaired older women. Nursing Research, 34, 140-144.

Mancini, J.A., \& Blieszner, R. (1992). Social provisions in adulthood: Concept and measurement in close relationships. Journal of Gerontology: Psychological Sciences, 47, P14-P20. 
Manton, K.G., Stallard, E., \& Corder, L.S. (1998). The dynamics of dimensions of agerelated disability 1982 to 1994 in the U.S. elderly population. Journals of Gerontology: Series A: Biological Sciences and Medical Sciences, 53, B59-B70. doi: 10.1093/gerona/53A.1.B59

Marsiske, M., Klumb, P., \& Baltes, M.M. (1997). Everyday activity patterns and sensory functioning in old age. Psychology and Aging, 12, 444-457.

Marsiske, M., Lang, F.R., Baltes, P.B. \& Baltes, M.M. (1995). Selective optimization with compensation: Life-span perspectives. In R.A. Dixon \& L. Backman (Eds.), Psychological compensation: Managing losses and promoting gains (pp. 35-79). Hillsdale, NJ: Erlbaum.

Marsland, A.L., Bachen, E.A., Cohen, S., \& Manuck, S.B. (2001). Stress, immunity, and susceptibility to infectious disease. In A. Baum, T.A. Revenson, \& J.E. Singer (Eds.), Handbook of health psychology (pp. 683-695). Mahwah, NJ: Erlbaum.

Martire, L.M., Stephens, M.A., Druley, J.A., \& Wojno, W.C. (2002). Negative reactions to received spousal care: Predictors and consequences of miscarried support. Health Psychology, 21, 167-176.

Mavandadi, S., Rook, K.S., \& Newsom, J.T. (2007). Positive and negative social exchanges and disability in later life: An investigation of trajectories of change. Journal of Gerontology: Social Sciences, 62B, S361-S370.

McClelland, G. H., \& Judd, C. M. (1993). Statistical difficulties of detecting interactions and moderator effects. Psychological Bulletin, 114, 376-390.

McCormack, J., McLeod, S., McAllister, L., \& Harrison, L.J. (2009). A systematic review of the association between childhood speech impairment and participation across the lifespan. International Journal of Speech-Language Pathology, 11, 155-170. doi: 10.1080/17549500802676859

McIlvane, J.M., \& Reinhardt, J.P. (2001). Interactive effect of support from family and friends in visually impaired elders. Journal of Gerontology: Psychological Sciences, 56B, P374-P382.

McIntosh, I. (1996). Interaction between professionals and older people: Where does the problem lie? Health Care in Later Life, 1, 29-38.

Meadows, L.M., \& Morse, J.M. (2000). Constructing evidence within the qualitative project. In J.M. Morse, J.M. Swansen, \& A. Kuzel (Eds.), Nature of qualitative evidence (pp.187-200). Thousand Oaks, CA: Sage.

Miller, P.M., Ingham, J.G., \& Davidson, S. (1976). Life events, symptoms, and social support. Journal of Psychosomatic Research, 20, 515-522. 
Mirowsky, J., \& Ross, C.E. (2007). Life course trajectories of perceived control and their relationship to education. American Journal of Sociology, 112, 1339-1382.

Morgan, D.L. (1989). Adjusting to widowhood: Do social networks really make it easier? The Gerontologist, 29, 101-107.

Morgan, D.L. (1998). Practical strategies for combining qualitative and quantitative methods: Applications to health research. Qualitative Health Research, 8, 362376.

Morgan, D. L. (2013). Integrating qualitative and quantitative methods: A pragmatic approach. Thousand Oaks, CA: SAGE Publications.

Morgan, D. L., Schuster, T. L., \& Butler, E. W. (1991). Role reversals in the exchange of social support. Journal of Gerontology: Social Sciences, 46, S278-S287.

Moustakas, C. (1994). Phenomenological research methods. Thousand Oaks, CA: SAGE Publications.

Mugford, S., \& Kendig, H. (1987). Social relations: Networks and ties. In H.L. Kendig (Ed.), Ageing and families: A social network perspective (pp. 38-59). Sydney: Allen \& Unwin.

Mullins, L.C. \& Duggan, E. (1990). The influence of depression, and family and friendship relations, on residents' loneliness in congregate housing. The Gerontologist, 30, 377-384.

Mulrow, C.D., Aguilar, C., Endicott, J.E., Tuley, M.R., Velez, R., Charlip, W.S., Rhodes, M.C., Hill, J.A., \& DeNino, L.A. (1990). Quality-of-life changes and hearing impairment: A randomized controlled trial. Annals of Internal Medicine, 113, 188-194.

Mulrow, C.D., Aguilar, C., Endicott, J.E., Velez, R., Tuley, M.R., Charlip, W.S., \& Hill, J.A. (1990). Association between hearing impairment and the quality of life of elderly individuals. Journal of the American Geriatrics Society. 38, 45-50.

Muramatsu, N. (2003). County-level income inequality and depression among older Americans. Health Services Research, 38, 1863-1884.

Murry, T., Cannito, M.P., \& Woodson, G.E. (1994). Spasmodic dysphonia: Emotional status and botulinum toxin treatment. Archives of Otolaryngology - Head \& Neck Surgery, 120, 310-6. doi: 10.1001/archotol.1994.01880270056010

Nachtegaal, J., Smit, J. H., Smits, C. A. S., Bezemer, P. D., van Beek, J. H., Festen, J. M., \& Kramer, S. E. (2009). The association between hearing status and psychosocial 
health before the age of 70 years: Results from an internet-based national survey on hearing. Ear \& Hearing, 30, 302-312.

Nagi, S. (1965). Some conceptual issues in disability and rehabilitation. In M. Sussman (Ed.), Sociology \& rehabilitation (pp. 100-113). Washington, DC: American Sociological Association.

Nagi, S.Z. (1976). An epidemiology of disability among adults in the United States. Millbank Memorial Fund Quarterly, 6, 493-508. doi: 10.2307/3349677

National Institute on Deafness and other Communication Disorders (NIDCD). (2006). Strategic plan: 2006-2008. Retrieved from http://www.nidcd.nih.gov/StaticResources/about/Plans/strategic/strategic0608.pdf

Neter, J., Kutner, M.H., \& Nachtsheim, C. J. (2004). Applied linear regression models, Fourth Edition. New York: McGraw-Hill Irwin.

Newsom, J.T. (1999). Another side to caregiving: Negative reactions to being helped. Current Directions in Psychological Science, 8, 183-187.

Newsom, J.T., Mahan, T.L., Rook, K.S., \& Krause, N. (2008). Stable negative social exchanges and health. Health Psychology, 27, 78-86.

Newsom, J.T., Nishishiba, M., Morgan, D.L., \& Rook, K.S. (2003). The relative importance of three domains of positive and negative social exchanges: A longitudinal model with comparable measures. Psychology \& Aging, 18, 746-754. doi: $10.1037 / 0882-7974.18 .4 .746$

Newsom, J.T., Rook, K.S., Nishishiba, M., Sorkin, D.H., \& Mahan, T.L. (2005). Understanding the relative importance of positive and negative social exchanges: Examining specific domains and appraisals. Journal of Gerontology: Psychological Sciences, 60B, P304-P312.

Newsom, J.T., \& Schulz, R. (1998). Caregiving from the percipients perspective: Negative reactions to being helped. Health Psychology, 17, 172-181.

Nunnally, J., \& Bernstein, L. (1994). Psychometric theory. New York: McGraw-Hill.

O'Connor, B.P. (1998). All-in-one programs for exploring interactions in moderated simple regression. Educational and Psychological Measurement, 58, 833-837.

Okun, M.A., Melichor, J.F., \& Hill, M.D. (1990). Negative daily events, positive and negative social ties, and psychological distress among older adults. The Gerontologist, 30, 193-199. doi: 10.1093/geront/30.2.193 
Oppegard, K., Hansson, R.O., Morgan, T., Indart, M., Crutcher, M., \& Hampton, P. (1984). Sensory loss, family support, and adjustment among the elderly. The Journal of Social Psychology, 123, 291-292.

Ormel, J., Kempen, G.I.J.M., Penninx, B.W.J.H., Brilman, E.I., Beekman, A.T.F., \& van Sonderen, E. (1997). Chronic medical conditions and mental health in older people: Disability and psychosocial resources mediate specific mental health effects. Psychological Medicine, 27, 1065-1077.

Pachana, N.A., Smith, N., Watson, M., McLaughlin, D., \& Dobson, A. (2008). Responsiveness of the Duke Social Support sub-scales in older women. Age and Ageing, 37, 666-672. doi: 10.1093/ageing/afn205

Palmer, A.D., \& Graham, M.S. (2004). The relationship between communication and quality of life in alaryngeal speakers. Journal of Speech-Language Pathology \& Audiology, 28, 6-24.

Palmer, A.D., Newsom, J.T., \& Rook, K.S. (2012). How do communication difficulties impact the social lives of older adults? Poster presented at the Gerontological Society of America, Annual Conference, San Diego, CA.

Parmelee, P.A., \& Lawton, M.P. (1990). The design of special environments for the aged. In J.E. Birren \& K.W. Schaie (Eds.), Handbook of the psychology of aging $\left(3^{\text {rd }}\right.$ ed., pp. 465-489). San Diego, CA: Academic Press.

Parr, S. (2007). Living with severe aphasia: Tracking social exclusion. Aphasiology, 21, 98-123. doi: 10.1080/02687030600798337

Pearlin, L.I., Mullan, J.T., Semple, S.J., \& Skaff, M.M. (1990). Caregiving and the stress process: An overview of concepts and their measures. The Gerontologist, 30, 583594.

Penley, J.A., Tomaka, J., \& Wiebe, J.S. (2002). The association of coping to physical and psychological health outcomes: A meta-analytic review. Journal of Behavioral Medicine, 25, 551-603.

Phillipson, C., Bernard, M., Phillips, J., \& Ogg, J. (1998). The family and community life of older people: Household composition and social networks in three suburban areas. Age \& Aging, 18, 259-288.

Philp, I., Lowles, R.V., Armstrong, G.K., \& Whitehead, C. (2002). Repeatability of standardized tests of functional impairment and well-being in older people in a rehabilitation setting. Disability \& Rehabilitation, 24, 243-249. 
Poissant, S.F., Beaudoin, F., Huang, J., Brodsky, J., \& Lee, D. (2008). Impact of cochlear implantation on speech understanding, depression, and loneliness in the elderly. Journal of Otolaryngology-Head \& Neck Surgery, 37, 488-494.

Polkinghorne, D.E. (1989). Phenomenological research methods. In R.S. Valle \& S. Halling (Eds.), Existential phenomenological perspectives in psychology (pp.4160). New York: Plenum Press.

Poortinga, W. (2006). Social relations or social capital? Individual and community health effects of bonding social capital. Social Science \& Medicine, 63, 255-270.

Preacher, K. J., \& Hayes, A. F. (2008). Asymptotic and resampling strategies for assessing and comparing indirect effects in multiple mediator models. Behavior Research Methods, 40, 879-891.

Preminger, J.E., \& Meeks, S. (2010). The influence of mood on the perception of hearing-loss related quality of life in people with hearing loss and their significant others. International Journal of Audiology, 49, 263-271. doi: $10.3109 / 14992020903311396$

Pronk, M., Deeg, D.D.H., Smits, C., van Tilburg, T.G., Kuik, D.J., Festen, J.M., \& Kramer, S.E. (2011). Prospective effects of hearing status on loneliness and depression in older persons: Identification of subgroups. International Journal of Audiology, 50, 887-896.

Putnam, M. (2002). Linking aging theory and disability models: Increasing the potential to explore aging with physical impairment. The Gerontologist, 42, 799-806.

Radloff, L. S. (1977). The CES-D scale: A self report depression scale for research in the general population. Applied Psychology and Measurement, 1, 385.

Reinhardt, J.P., Boerner, K., \& Horowitz, A. (2006). Good to have but not to use: Differential impact of perceived and received support on well-being. Journal of Social and Personal Relationships, 23, 117-129.

Resnick, H.E., Fries, B.E., Verbrugge, L.M. (1997). Windows to their world: The effect of sensory impairments on social engagement and activity time in nursing home residents. Journal of Gerontology: Social Sciences, 52B, S135-S144.

Reynolds, J.S., \& Perrin, N.A. (2004). Mismatches in social support and psychosocial adjustment to breast cancer. Health Psychology, 23, 425-430.

Romppel, M., Herrmann-Lingen, C., Wachter, R., Edelmann, F., Dungen, H.-D., Pieske, B., \& Grande, G., (2013). A short form of the General Self-Efficacy Scale (GSE6): Development, psychometric properties and validity in an intercultural non- 
clinical sample and a sample of patients at risk for heart failure. GMS PsychoSocial Medicine, 10, 1-7. ISSN 1860-5214.

Rook, K.S. (1984). The negative side of social interaction. Journal of Personality and Social Psychology, 52, 1132-1147.

Rook, K.S. (1987). Social support versus companionship: Effects on life stress, loneliness and evaluations by others. Journal of Personality and Social Psychology, 52. 1132-1147.

Rook, K.S. (1998). Investigating the positive and negative sides of personal relationships: Through a glass darkly? In B.H.Spitzberg \& W.R. Cupach (Eds.), The dark side of close personal relationships (pp. 369-393). Mahwah, NJ: Erlbaum.

Rosow, I., \& Breslau, N. (1966). A Guttman health scale for the aged. Journal of Gerontology, 21, 556-559. doi: 10.1093/geronj/21.4.556

Rotter, J.B. (1966). Generalized expectancies for internal versus external control of reinforcement. Psychological Monographs: General and Applied, 80, 1-28.

Rowe, J.W., \& Kahn, R.L. (1998). Successful aging. New York: Pantheon Books.

Roy, C. (1976). Introduction to nursing: An adaptation model. Englewood, NJ: PrenticeHall.

Ruben, R.J. (2000). Redefining the survival of the fittest: Communication disorders in the 21st century. Laryngoscope, 110, 241-5. doi: 10.1097/00005537-20000201000010

Rudberg, M.A., Furner, S.E., Dunn, J.E., \& Cassel, C.K. (1993). The relationship of visual and hearing impairments to disability: An analysis using the longitudinal study of aging. Journal of Gerontology, 48, M261-M265.

Russell, D.W. (1996). UCLA Loneliness Scale (Version 3): Reliability, validity, and factor structure. Journal of Personality Assessment, 66, 20-40.

Russell, D.W., \& Cutrona, C.E. (1991). Social support, stress, and depressive symptoms among the elderly: Test of a process model. Psychology \& Aging, 6, 190-201.

Russell, D.W., Cutrona, C.E., Rose, J., \& Yurko, K. (1984). Social and emotional loneliness: An examination of Weiss's typology of loneliness. Journal of Personality and Social Psychology, 46, 1313-1321.

Russell, D. W., Cutrona, C. E., de la Mora, A., \& Wallace, R. B. (1997). Loneliness and nursing home admission among rural older adults. Psychology and Aging, 12, 574. 
Ruth, J.E., \& Coleman, P. (1996). Personality and aging: Coping and management of the self in later life. In J.E. Birren \& K.W. Schaie (Eds.), Handbook of the psychology of aging ( $4^{\text {th }}$ ed.). San Diego: Academic Press.

Ryan, E.B., Giles, H., Bertolucci, G., \& Henwood, K. (1986). Psycholinguistic and social psychological components of communication by and with the elderly. Language and Communication, 6, 1-24.

Salthouse, T.A. (1990). Cognitive competence and expertise in aging. In J.E. Birren \& K.W. Schaie (Eds.), Handbook of the psychology of aging ( $3^{\text {rd }}$ ed., pp. 310-391). New York: Academic Press.

Santor, D.A. \& Coyne, J.C. (1997). Shortening the CES-D to improve its ability to detect cases of depression. Psychological Assessment, 9, 233-243.

Sarason, I.G., \& Sarason, B.R. (1986). Experimentally provided social support. Journal of Personality \& Social Psychology, 50, 1222-1225.

Saunders, G.H., \& Echt, K. (2007). An overview of dual sensory impairment in older adults: Perspectives for rehabilitation. Trends in Amplification, 11, 243-258. doi: $10.1177 / 1084713807308365$

Scarinci, N., Worrall, L., \& Hickson, L. (2008). The effect of hearing impairment in older people on the spouse. International Journal of Audiology, 47, 141-151. doi: $10.1080 / 14992020701689696$

Schaefer, C., Coyne, J.C., \& Lazarus, R.S. (1981). The health-related functions of social support. Journal of Behavioral Medicine, 4, 381-406.

Scherer, M.J., \& Frisina, D.R. (1998). Characteristics associated with marginal hearing loss and subjective well-being among a sample of older adults. Journal of Rehabilitation Research \& Development, 35, 420-426.

Schneider, J., Gopinath, B., Karpa, M. J., McMahon, C. M., Rochtchina, E., Leeder, S. R., \& Mitchell, P. (2010). Hearing loss impacts on the use of community and informal supports. Age and Ageing, 39, 458-464. doi: 10.1093/ageing/afq051

Seaman, M.A., Levin, J.R., \& Serlin, R.C. (1991). New developments in pairwise multiple comparisons: Some powerful and practicable procedures. Psychological Bulletin, 110, 577-586.

Seeman, T.E. (1996). Social ties and health: The benefits of social integration. Annals of Epidemiology, 6, 442-451. 
Seeman, T.E., Bruce, M.L., \& McAvay, G.J. (1996). Social networks and onset of ADL disability: MacArthur Studies of Successful Aging. Journal of Gerontology, Social Sciences, 51B, S191-S200.

Shadden, B.B. (1988). Communication behavior and aging: A sourcebook for clinicians. Baltimore, MD: Williams \& Wilkins.

Shadden, B.B. (1997). Discourse behaviors in older adults. Seminars in Speech \& Language, 18, 143-156.

Shanas, E. (1979). Social myth as hypothesis: The case of family relations of old people. The Gerontologist, 19, 3-9.

Sherer, M., Maddux, J.E., Mercandante, B., Prentice-Dunn, S., Jacobs, B., \& Rogers, R.W. (1982). The self-efficacy scale: Construction and validation. Psychological Reports, 51, 663-671.

Shortridge-Baggett, L. M. (2001). Self-efficacy: Measurement and intervention in nursing. Scholarly Inquiry for Nursing Practice: An International Journal, 15, 183-188.

Shrout, P.E., \& Bolger, N. (2002). Mediation in experimental and nonexperimental studies: New procedures and recommendations. Psychological Methods, 7, 422445.

Silverstein, M., Conroy, S. J., Wang, H., Giarrusso, R., \& Bengtson, V. L. (2002). Reciprocity in parent-child relations over the adult life course. The Journals of Gerontology Series B: Psychological Sciences and Social Sciences, 57, S3-S13.

Siupsinskiene, N., Razbadauskas, A., \& Dubosas, L. (2011). Psychological distress in patients with benign voice disorders. Folia Phonatrica Logopedica, 63, 281-288.

Skinner, E.A., Edge, K., Altman, J., \& Sherwood, H. (2003). Searching for the structure of coping: A review and critique of category systems for classifying ways of coping. Psychological Bulletin, 129, 216-269.

Smith, J., \& Baltes, M.M. (1998). The role of gender in very old age: Profiles of functioning and everyday life patterns. Psychology \& Aging, 13, 676-695.

Smith, S.L. \& West, R.L. (2006). The application of self-efficacy principles to audiologic rehabilitation: A tutorial. American Journal of Audiology, 15, 46-56.

Sorkin, D.H., \& Rook, K.S. (2004). Interpersonal control strivings and vulnerability to negative social exchanges in later life. Psychology and Aging, 19, 555-564. 
Starks, H., Morris, M.A., Yorkston, K.M., Gray, R.F., \& Johnson, K.L. (2010). Being inor out-of-sync: Couples' adaptation to change in multiple sclerosis. Disability and Rehabilitation, 32, 196-206.

Stephens, D., \& Kerr, P. (2003). The role of positive experiences in living with an acquired hearing loss. International Journal of Audiology, 42, S118-S127.

Stoller, E.P., \& Earl, L.L. (1983). Help with activities of everyday life: Sources of support for the noninstitutionalized elderly. The Gerontologist, 23, 64-70.

Strawbridge W.J., Wallhagen M.I., Shema S.J \& Kaplan G.A. (2000). Negative consequences of hearing impairment in old age: A longitudinal analysis. The Gerontologist, 40, 320-326.

Tashakkori, A., \& Teddlie, C. (1998). Mixed methodology: Combining qualitative and quantitative approaches. Thousand Oaks, CA: SAGE.

Taylor, S.E., \& Stanton, A. (2007). Coping resources, coping processes, and mental health. Annual Review of Clinical Psychology, 3, 129-153.

Teddlie, C., \& Tashakkori, A. (2003). Major issues and controversies in the use of mixed methods in the social and behavioral sciences. In A. Tashakkori \& C. Teddlie (Eds.), Handbook of mixed methods in social \& behavioral research (pp. 3-50). Thousand Oaks, CA: Sage Publications.

Thibaut, J., \& Kelley, H.H. (1959). The social psychology of groups. New York: Wiley.

Threats, T.T. (2006). Towards an international framework for communication disorders: Use of the ICF. Journal of Communication Disorders, 39, 251-265.

Tobin, S.S., \& Kulys, R. (1981). The family in the institutionalization of the elderly. Journal of Social Issues, 37, 133-144.

Tolson, D., \& McIntosh, J. (1997). Listening in the care environment - Chaos or clarity for the hearing-impaired elderly person. International Journal of Nursing Studies, $34,173-182$.

Tolson, D., Swan, I., \& Knussen, C. (2002). Hearing disability: A source of distress for older people and carers. British Journal of Nursing, 11, 1021-1025.

Townsend, P. (1963). The family life of old people: An inquiry in East London. London, UK: Penguin Books.

Uchino, B.N. (2004). Social support and physical health: Understanding the health consequences of relationships. New Haven, CT: Yale University Press. 
Uchino, B.N. (2006). Social support and health: A review of physiological processes potentially underlying links to disease outcomes. Journal of Behavioral Medicine, 29, 377-387.

Uchino, B.N., Cacioppo, J.T., \& Kiecolt-Glaser, J.K. (1996). The relationship between social support and physiological processes: A review with emphasis on underlying mechanisms and implications for health. Psychological Bulletin, 119, $488-531$.

U.S. Census Bureau (2014). State and county quick facts. Washington, D.C.: U.S. Census Bureau. Available from: http://quickfacts.census.gov/

van Baarsen, B., Snijders, T.A.B., Smit, J.H., \& van Duijn, M.A.J. (2001). Lonely but not alone: Emotional isolation and social isolation as two distinct dimensions of loneliness in older people. Educational and Psychological Measurement, 61, 119135 .

van Leer, E., Hapner, E.R., \& Connor, N.P. (2008). Transtheoretical model of health behavior change applied to voice therapy. Journal of Voice, 22, 688-98.

van Manen, M. (1990). Researching lived experience. New York: State University of New York Press.

van Tilburg, T. (1998). Losing and gaining in old age: Changes in personal network size and social support in a four-year longitudinal study. Journal of Gerontology, 53B, S313-S323.

van Tilburg, T., \& Thomese, F. (2010). Societal dynamics in personal networks. In D. Dannefer \& C. Phillipson (Eds.), The SAGE handbook of social gerontology (pp.215-225). Thousand Oaks, CA: SAGE.

Ventry, I., \& Weinstein, B. (1982). The Hearing Handicap Inventory for the Elderly: A new tool. Ear and Hearing, 3, 128-134.

Ventry, I.M., \& Weinstein, B.E. (1983). Identification of elderly people with hearing problems. ASHA, 25, 37-42.

Verbrugge, L. M., \& Jette, A. M. (1994). The disablement process. Social science \& medicine, 38, 1-14.

Victor, C., Scambler, S., Bond, J., \& Bowling, A. (2000). Being alone in later life: loneliness, social isolation and living alone. Reviews in Clinical Gerontology, 10, $407-417$

Vingerhoets, G., Lannoo, E., van der Linden, C., Caemaert, J., Vandewalle, V., van den Abbeele, D., \& Wolters, M. (1999). Changes in quality of life following unilateral 
pallidal stimulation in Parkinson's disease. Journal of Psychosomatic Research, $46,247-55$.

Waite, L.J., Laumann, E.O., Levinson, W., Lindau, S.T., McClintock, M.K., O'Muircheartaigh, C.A., \& Schumm, L.P. (2007). National social life, health and aging project (NSHAP): Data collection instruments. Ann Arbor, MI: InterUniversity Consortium for Political and Social Research.

Wallhagen, M.I. (2002). Hearing impairment. Annual Review of Nursing Research, 20, 341-68.

Wallhagen M.I., Strawbridge W.J. \& Kaplan G.A. (1996). 6-year impact of hearing impairment on psychosocial and physiologic functioning. Nursing Practice, 21, $11-14$.

Wallhagen, M.I., Strawbridge, W.J., Shema, S.J., \& Kaplan, G.A. (2004). Impact of selfassessed hearing loss on a spouse: A longitudinal analysis of couples. The Journals of Gerontology, Series B: Psychological Sciences and Social Sciences, 59, S190-S196. doi: 10.1093/geronb/59.3.S190

Wallston, K.A., Wallston, B.S., Smith, S., \& Dobbins, C.J. (1987). Perceived control and health. Current Psychological Research and Reviews, 6, 15-25.

Walshe, M., \& Miller, N. (2011). Living with acquired dysarthria: The speaker's perspective. Disability \& Rehabilitation, 33, 195-203.

Wancata, J., Alexandrowicz, R., Marquart, B., Weiss, M., \& Friedrich, F. (2006). The criterion validity of the Geriatric Depression Scale: A systematic review. Acta Psychiatrica Scandinavica, 114, 398-410.

Ware, J.E., \& Sherbourne, C.D. (1992). The MOS 36-item short form health survey (SF36): Conceptual framework and item selection. Medical Care, 30 (6), 473-483. doi: 10.1097/00005650-199206000-00002

Washington Group on Disability Statistics (2011). Extended question set on functioning (WG ES-F). Atlanta, GA: Centers for Disease Control. Retrieved from: http://www.cdc.gov/nchs/data/washington_group/WG_Extended_Question_Set_o n_Functioning.pdf

Weinstein, B.E., \& Ventry, I.M. (1982). Hearing impairment and social isolation in the elderly. Journal of Speech and Hearing Research, 25, 593-599.

Weiss, R.W. The provisions of social relationships. (1974). In Z. Rubin (Ed.) Doing unto others. Englewood Cliffs, NJ: Prentice-Hall. 
Wells, M. (2009). Resilience in community-dwelling older adults. Journal of Rural Health, 25, 415-419.

Whitbeck, L. B., Simons, R. L., \& Conger, R. D. (1991). The effects of early family relationships on contemporary relationships and assistance patterns between adult children and their parents. Journal of Gerontology: Social Sciences, 46, S301S337.

Wood, V., \& Robertson, J.F. (1978). Friendship and kinship interaction: Differential effect on the morale of the elderly. Journal of Marriage and the Family, 40, 367375.

World Health Organization. (1980). ICIDH: International Classification of Impairments, Disabilities, and Handicaps. Geneva, Switzerland: WHO.

World Health Organization. (2001). ICF: International Classification of Functioning, Disability and Health. Geneva, Switzerland: WHO.

World Health Organization. (2002). Active ageing: A policy framework. Madrid, Spain: WHO.

Worrall, L., \& Hickson, L. (2003). Communication disability in aging: Prevention to intervention. Mason, $\mathrm{OH}$ : Cengage Learning.

Wright, D.L., \& Aquilino, W.S. (1998). Influence of emotional support exchange in marriage on caregiving wives' burden and marital satisfaction. Family Relations, 47, $195-204$.

Yancura, L.A., \& Aldwin, C.M. (2008). Coping and health in older adults. Current Psychiatry Reports, 10, 10-15.

Yip, P.S.F., Chi, I., Chiu, H., Wai, K.C., Conwell, Y., \& Caine, E. (2003). Prevalence study of suicide ideation among older adults in Hong Kong SAR. International Journal of Geriatric Psychiatry, 18, 1056-1062. doi: 10.1002/gps.1014

Yorkston, K.M., Beukelman, D.R., Strand, E.A., \& Bell, K.R. (Eds.) (1999). Management of motor speech disorders in children and adults. Austin, TX: ProEd.

Yorkston, K.M., Bourgeois, M.S., \& Baylor, C.R. (2010). Communication and aging. Physical Medicine \& Rehabilitation Clinics of North America, 21, 309-319.

Zhan, L. (2000). Cognitive adaptation and self-consistency in hearing impaired older persons: Testing Roy's Adaptation Model. Nursing Science Quarterly, 13, 158165. 
Appendix A. Summary of Published Instruments 


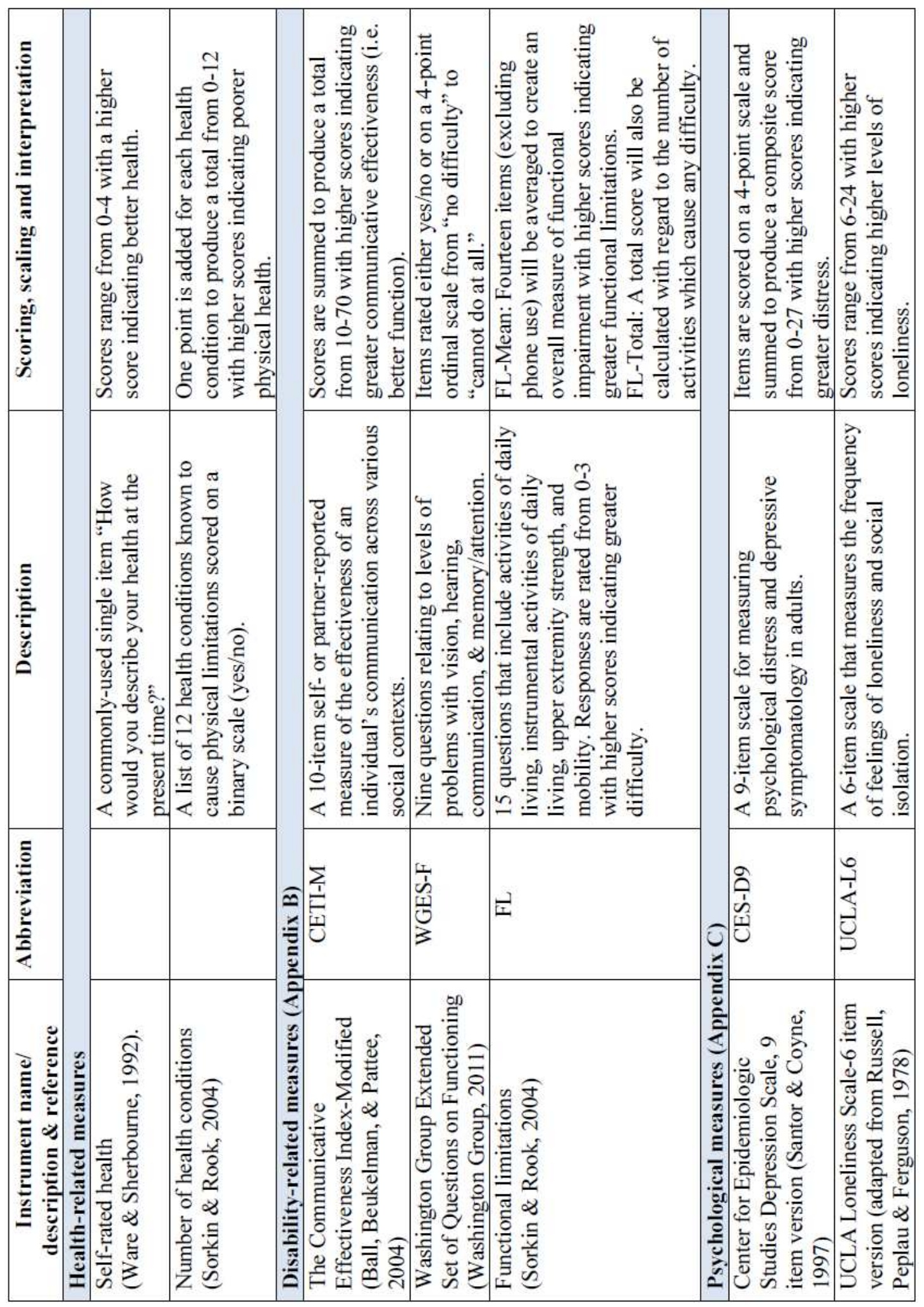




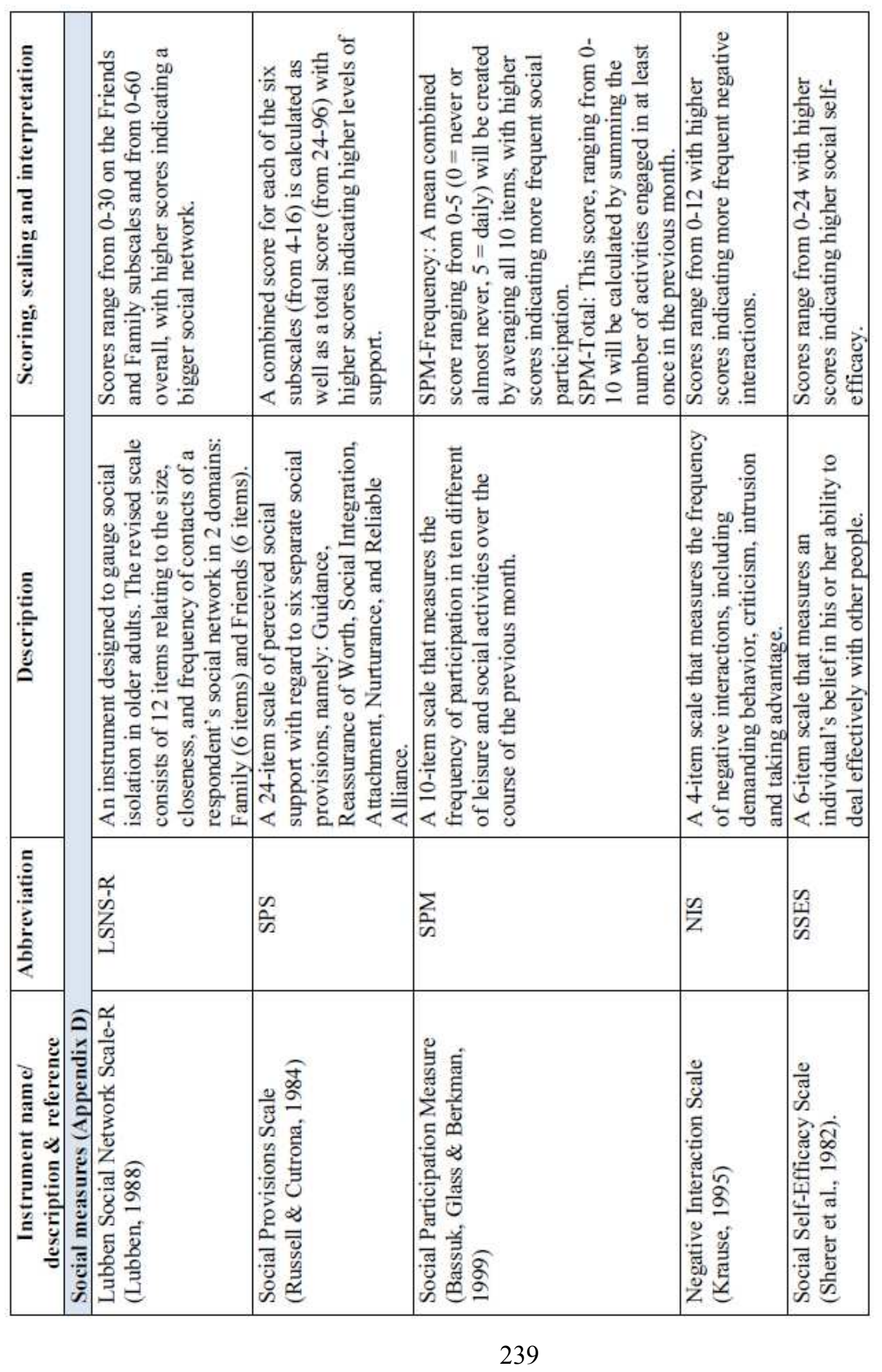


Appendix B. Published Instruments Related to Disability 


\title{
$\underline{\text { The Communicative Effectiveness Index-Modified (CETI-M) }}$
}

Person Completing the Survey:

\author{
- Speaker with ALS \\ - Family/Friend/Caregiver
}

Please evaluate how effectively the speaker communicates in these situations. Read the item describing each of the situations and decide how successful the speaker communicates. If you think that communication is very effective, circle the 7. If communication doesn't occur at all, circle the 1 . Circle any number on the scale that best describes communication in that situation.

1. Having a conversation with familiar persons in a quiet environment.

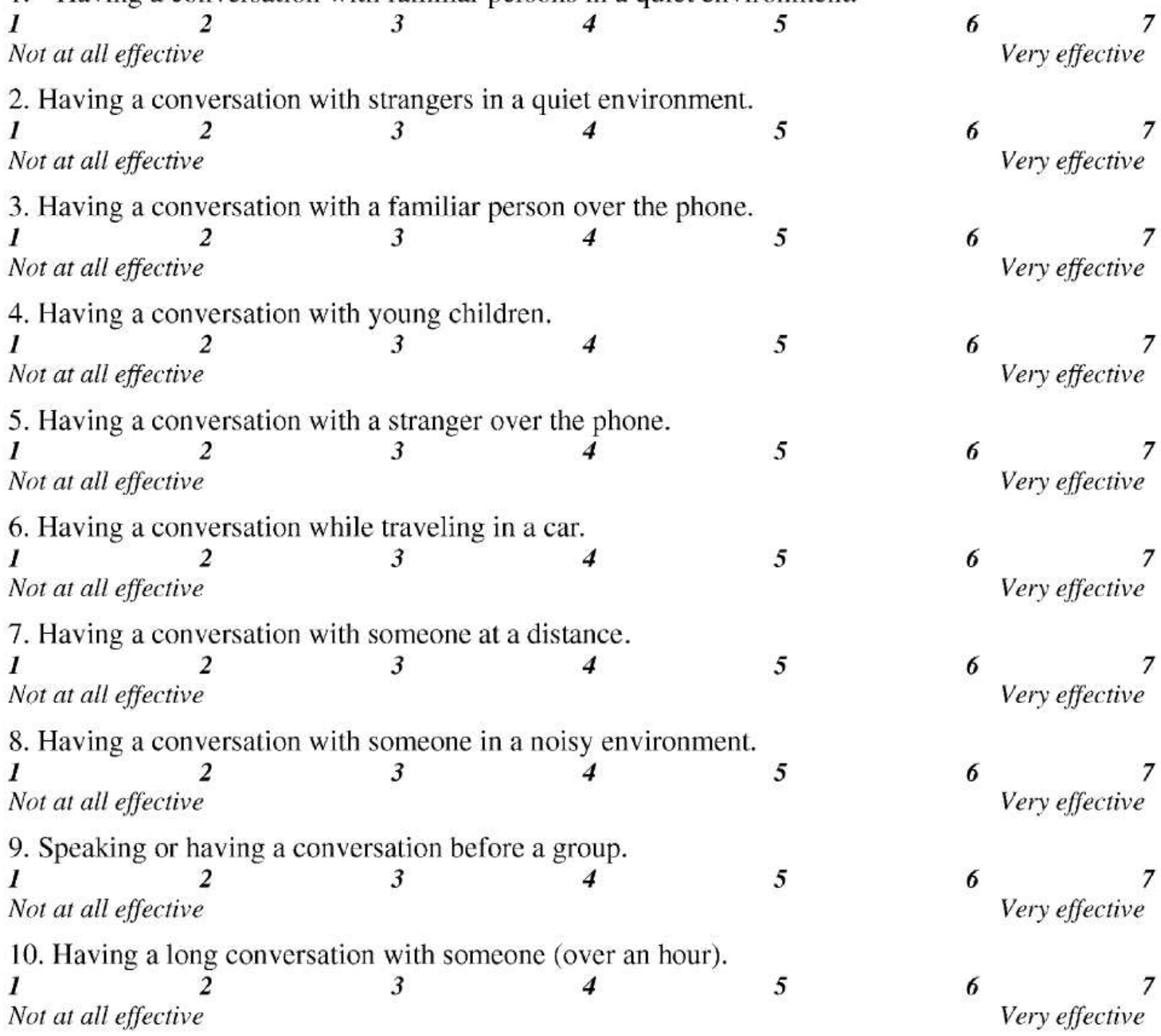




\section{Questions Related to Vision, Hearing, Communication \& Cognition from the}

\section{Washington Group Extended Question Set on Functioning (WG ES-F)}

INSTRUCTIONS: The following questions ask about your ability to do different activities.

1. Do you wear glasses?
a. Yes

b. No

2. Do you have difficulty seeing, even if wearing glasses?
a. No difficulty
b. Some difficulty
c. A lot of difficulty
d. Cannot do at all/Unable to do

3. Do you use a hearing aid?
a. Yes
b. No

4. Do you have difficulty hearing, even if using a hearing aid?
a. No difficulty
b. Some difficulty
c. A lot of difficulty
d. Cannot do at all/Unable to do

5. Using your usual language, do you have difficulty communicating, for example understanding or being understood?
a. No difficulty
b. Some difficulty
c. A lot of difficulty
d. Cannot do at all/Unable to do

6. Do you use Sign Language?
a. Yes
b. No

7. Do you have difficulty remembering or concentrating?
a. No difficulty
b. Difficulty remembering only
c. Difficulty concentrating only
d. Difficulty with both remembering and concentrating

8. How often do you have difficulty remembering? Would you say
a. Never
b. Sometimes
c. Often
d. All of the time

9. Do you have difficulty remembering a few things, a lot of things, or almost everything?
a. Nothing
b. A few things
c. A lot of things
d. Almost everything 
REFERENCE: Washington Group on Disability Statistics (2011). Extended question set on functioning (WG ES-F). Atlanta, GA: Centers for Disease Control. Retrieved from: http://www.cdc.gov/nchs/data/washington_group/WG_Extended_Question_Set_on_Functioning. pdf 
Appendix C. Published Instruments Related to Mental Health \& Well-Being 


\section{Center for Epidemiologic Studies Depression Scale (CES-D), 9 item version}

INSTRUCTIONS: Below is a list of the ways you might have felt or behaved. Please tell me how often you have felt this way during the past week.

\begin{tabular}{|c|c|c|c|c|}
\hline During the past week... & $\begin{array}{l}\text { Rarely or } \\
\text { none of } \\
\text { the time } \\
\text { (less than } \\
1 \text { day) }\end{array}$ & $\begin{array}{l}\text { Some or } \\
\text { a little of } \\
\text { the time } \\
(1-2 \\
\text { days })\end{array}$ & $\begin{array}{l}\text { Occasion } \\
\text {-ally or a } \\
\text { moderate } \\
\text { amount } \\
\text { of the } \\
\text { time } \\
\text { (3-4 } \\
\text { days) }\end{array}$ & $\begin{array}{c}\text { Most or } \\
\text { all of the } \\
\text { time } \\
\text { (5-7 } \\
\text { days) }\end{array}$ \\
\hline $\begin{array}{l}\text { 1. I was bothered by things that } \\
\text { usually don't bother me. }\end{array}$ & $\mathrm{O}_{0}$ & $\mathrm{O}_{1}$ & $\mathrm{O}_{2}$ & $\mathrm{O}_{3}$ \\
\hline $\begin{array}{l}\text { I felt that I could not shake off the } \\
\text { 2. blues even with help from my } \\
\text { family or friends. }\end{array}$ & $\mathrm{O}_{0}$ & $\mathrm{O}_{1}$ & $\mathrm{O}_{2}$ & $\mathrm{O}_{3}$ \\
\hline $\begin{array}{l}\text { 3. I had trouble keeping my mind on } \\
\text { what I was doing. }\end{array}$ & O0 & $\mathrm{O}_{1}$ & $\mathrm{O}_{2}$ & $\mathrm{O}_{3}$ \\
\hline 4. I felt depressed. & O0 & $\mathrm{O}_{1}$ & $\mathrm{O}_{2}$ & $\mathrm{O}_{3}$ \\
\hline $\begin{array}{l}\text { 5. I felt that everything I did was an } \\
\text { effort. }\end{array}$ & O0 & $\mathrm{O}_{1}$ & $\mathrm{O}_{2}$ & $\mathrm{O}_{3}$ \\
\hline 6. My sleep was restless. & $\bigcirc_{0}$ & $\bigcirc_{1}$ & $\mathrm{O}_{2}$ & $\mathrm{O}_{3}$ \\
\hline 7. I was happy. & $\bigcirc_{3}$ & $\mathrm{O}_{2}$ & $\mathrm{O}_{1}$ & $\mathrm{O}_{0}$ \\
\hline 8. I enjoyed life. & $\bigcirc_{3}$ & $\mathrm{O}_{2}$ & $\mathrm{O}_{1}$ & $\mathrm{O}_{0}$ \\
\hline 9. I felt sad. & Oo & $\mathrm{O}_{1}$ & $\mathrm{O}_{2}$ & $\mathrm{O}_{3}$ \\
\hline
\end{tabular}

SCORING: zero for answers in the first column, 1 for answers in the second column, 2 for answers in the third column, 3 for answers in the fourth column. The scoring of positive items $(7 \& 8)$ is reversed. Possible range of scores is zero to 27 , with the higher scores indicating the presence of more symptomatology.

REFERENCE: Santor, D.A. \& Coyne, J.C. (1997). Shortening the CES-D to improve its ability to detect cases of depression. Psychological Assessment, 9, 233-243. 


\section{UCLA Loneliness Scale (6 item version)}

Below are some statements that might describe ways that you feel. Please put an ' $\mathrm{X}$ ' in the box that indicates how strongly you agree or disagree with each phrase.

\begin{tabular}{|c|c|c|c|c|}
\hline How often do you feel... & Never & Rarely & $\begin{array}{l}\text { Some- } \\
\text { times }\end{array}$ & Always \\
\hline a. Isolated from others? & $\bigcirc_{1}$ & $\mathrm{O}_{2}$ & $\mathrm{O}_{3}$ & $\mathrm{O}_{4}$ \\
\hline *b. That you belong to a group of friends? & $\mathrm{O}_{4}$ & $\mathrm{O}_{3}$ & $\mathrm{O}_{2}$ & $\mathrm{O}_{1}$ \\
\hline c. That no one really knows you well? & $\mathrm{O}_{1}$ & $\mathrm{O}_{2}$ & $\mathrm{O}_{3}$ & $\mathrm{O}_{4}$ \\
\hline $\begin{array}{l}\text { d. That your relationships with others are not } \\
\text { meaningful? }\end{array}$ & $\mathrm{O}_{1}$ & $\mathrm{O}_{2}$ & $\mathrm{O}_{3}$ & $\mathrm{O}_{4}$ \\
\hline $\begin{array}{l}\text { *e. That there are people who really understand } \\
\text { you? }\end{array}$ & $\bigcirc_{4}$ & $\mathrm{O}_{3}$ & $\mathrm{O}_{2}$ & $O_{1}$ \\
\hline f. That you lack companionship? & $\mathrm{O}_{1}$ & $\mathrm{O}_{2}$ & $\mathrm{O}_{3}$ & $\mathrm{O}_{4}$ \\
\hline
\end{tabular}

SCORING: Items that are asterisked should be reversed (i.e., $1=4,2=3,3=2,4=1$ ), and the scores for each item then summed together to produce a total from 6-24. Higher scores indicate greater degrees of loneliness.

REFERENCE: Russell, D.W. (1996). UCLA Loneliness Scale (Version 3): Reliability, validity, and factor structure. Journal of Personality Assessment, 66, 20-40. 
Appendix D. Published Instruments Related to Social Relationships 


\section{$\underline{\text { Lubben Social Network Scale-Revised (LSNS-R) }}$}

FAMILY: Considering the people to whom you are related by birth, marriage, adoption, etc...

1. How many relatives do you see or hear from at least once a month?

$$
0 \text { =none } \quad 1 \text { =one } \quad 2 \text { =two } 3 \text { =three or four } \quad 4 \text { =five thru eight } 5=\text { nine or more }
$$

2. How often do you see or hear from the relative with whom you have the most contact?

$$
\begin{aligned}
& \begin{array}{l}
0 \\
=
\end{array}=\text { less than monthly } \quad 1=\text { monthly } \quad 2=\text { few times } a \text { month } \quad 3=\text { weekly } \quad 4=\text { few times a week } \\
& 5=\text { daily }
\end{aligned}
$$

3. How many relatives do you feel at ease with that you can talk about private matters?

$$
0=\text { none } \quad 1 \text { =one } \quad 2 \text { =two } 3 \text { =three or four } \quad 4 \text { =five thru eight } 5=\text { nine or more }
$$

4. How many relatives do you feel close to such that you could call on them for help?

$$
0=\text { none } \quad 1 \text { =one } 2 \text { =two } 3 \text { =three or four } 4 \text { =five thru eight } 5=\text { nine or more }
$$

5. When one of your relatives has an important decision to make, how often do they talk to you about it?

$$
0=\text { never } \quad 1 \text { =seldom } \quad 2 \text { =sometimes } \quad 3 \text { =often } \quad 4 \text { =very often } 5 \text { =always }
$$

6. How often is one of your relatives available for you to talk to when you have an important decision to make?

$$
0=\text { never } \quad 1=\text { seldom } \quad 2 \text { =sometimes } \quad 3 \text { =often } \quad 4=\text { very often } \quad 5=\text { always }
$$

FRIENDSHIPS: Considering all of your friends including those who live in your neighborhood...

7. How many of your friends do you see or hear from at least once a month?

$$
0=\text { none } \quad 1 \text { =one } \quad 2 \text { =two } 3 \text { =three or four } \quad 4 \text { =five thru eight } 5 \text { = nine or more }
$$

8. How often do you see or hear from the friend with whom you have the most contact? $0=$ less than monthly $\quad 1=$ monthly $\quad 2$ =few times $a$ month $\quad 3=$ weekly $\quad 4=$ few times a week $5=$ daily

9. How many friends do you feel at ease with that you can talk about private matters? $0=$ none $\quad 1$ =one $\quad 2$ =two $\quad 3$ =three or four $\quad 4$ =five thru eight $\quad 5$ = nine or more

10. How many friends do you feel close to such that you could call on them for help?

$$
0=\text { none } \quad 1 \text { =one } \quad 2 \text { =two } 3 \text { =three or four } \quad 4 \text { =five thru eight } 5=\text { nine or more }
$$

11. When one of your friends has an important decision to make, how often do they talk to you about it?

$$
0 \text { never } \quad 1 \text { =seldom } \quad 2 \text { =sometimes } \quad 3 \text { =often } \quad 4 \text { =very often } \quad 5 \text { =always }
$$

12. How often is one of your friends available for you to talk to when you have an important decision to make?

$$
0=\text { never } \quad 1 \text { =seldom } \quad 2 \text { =sometimes } \quad 3 \text { =often } \quad 4=\text { very often } \quad 5=\text { always }
$$

LSNS-R total score is an equally weighted sum of these twelve items. Scores range from 0 to 60. 


\section{Social Provisions Scale}

INSTRUCTIONS: In answering the following questions, think about your current relationships with friends, family members, co-workers, community members and so on. Please indicate to what extent each statement describes your current relationships with other people. Use the following scale to indicate your opinion.

\section{STRONGLY DISAGREE DISAGREE AGREE STRONGLYAGREE 1 2 3 4}

So, for example, if you feel a statement is very true of your current relationships, you would respond with a 4 (strongly agree). If you feel a statement clearly does not describe your relationships, you would respond with a 1 (strongly disagree).

\begin{tabular}{|c|c|c|c|c|c|}
\hline & & $\begin{array}{l}\text { Strongly } \\
\text { Disagree }\end{array}$ & Disagree & Agree & $\begin{array}{l}\text { Strongly } \\
\text { Agree }\end{array}$ \\
\hline 1 & $\begin{array}{l}\text { There are people I can depend on } \\
\text { to help me if I really need it. }\end{array}$ & $\mathrm{O}_{1}$ & $\mathrm{O}_{2}$ & $\bigcirc_{3}$ & $\mathrm{O}_{4}$ \\
\hline 2 & $\begin{array}{l}\text { I feel that I do not have close } \\
\text { personal relationships with other } \\
\text { people. }\end{array}$ & $\mathrm{O}_{4}$ & $\mathrm{O}_{3}$ & $\mathrm{O}_{2}$ & $\mathrm{O}_{1}$ \\
\hline 3 & $\begin{array}{l}\text { There is no one I can turn to for } \\
\text { guidance in times of stress. }\end{array}$ & $\mathrm{O}_{4}$ & $\mathrm{O}_{3}$ & $\mathrm{O}_{2}$ & $\bigcirc_{1}$ \\
\hline 4 & $\begin{array}{l}\text { There are people who depend on } \\
\text { me for help. }\end{array}$ & $\mathrm{O}_{1}$ & $\mathrm{O}_{2}$ & $\mathrm{O}_{3}$ & $\mathrm{O}_{4}$ \\
\hline 5 & $\begin{array}{l}\text { There are people who enjoy the } \\
\text { same social activities I do. }\end{array}$ & $\mathrm{O}_{1}$ & $\mathrm{O}_{2}$ & $\mathrm{O}_{3}$ & $\mathrm{O}_{4}$ \\
\hline 6 & $\begin{array}{l}\text { Other people do not view me as } \\
\text { competent. }\end{array}$ & $\mathrm{O}_{4}$ & $\mathrm{O}_{3}$ & $\mathrm{O}_{2}$ & $\mathrm{O}_{1}$ \\
\hline 7 & $\begin{array}{l}\text { I feel personally responsible for } \\
\text { the well-being of another person. }\end{array}$ & $\mathrm{O}_{1}$ & $\mathrm{O}_{2}$ & $\mathrm{O}_{3}$ & $\mathrm{O}_{4}$ \\
\hline 8 & $\begin{array}{l}\text { I feel part of a group of people } \\
\text { who share my attitudes and } \\
\text { beliefs. }\end{array}$ & $\mathrm{O}_{1}$ & $\mathrm{O}_{2}$ & $\mathrm{O}_{3}$ & $\bigcirc_{4}$ \\
\hline 9 & $\begin{array}{l}\text { I do not think other people respect } \\
\text { my skills and abilities. }\end{array}$ & $\mathrm{O}_{4}$ & $\mathrm{O}_{3}$ & $\mathrm{O}_{2}$ & $\mathrm{O}_{1}$ \\
\hline 10 & $\begin{array}{l}\text { If something went wrong, no one } \\
\text { would come to my assistance. }\end{array}$ & $\mathrm{O}_{4}$ & $\mathrm{O}_{3}$ & $\mathrm{O}_{2}$ & $\bigcirc_{1}$ \\
\hline 11 & $\begin{array}{l}\text { I have close relationships that } \\
\text { provide me with a sense of } \\
\text { emotional security and well-being. }\end{array}$ & $\mathrm{O}_{1}$ & $\mathrm{O}_{2}$ & $\mathrm{O}_{3}$ & $\mathrm{O}_{4}$ \\
\hline 12 & $\begin{array}{l}\text { There is someone I could talk to } \\
\text { about important decisions in my } \\
\text { life. }\end{array}$ & $O_{1}$ & $\mathrm{O}_{2}$ & $\mathrm{O}_{3}$ & $\mathrm{O}_{4}$ \\
\hline
\end{tabular}




\begin{tabular}{|c|c|c|c|c|c|}
\hline & & $\begin{array}{l}\text { Strongly } \\
\text { Disagree }\end{array}$ & Disagree & Agree & $\begin{array}{l}\text { Strongly } \\
\text { Agree }\end{array}$ \\
\hline 13 & $\begin{array}{l}\text { I have relationships where my } \\
\text { competence and skill are } \\
\text { recognized. }\end{array}$ & $O_{1}$ & $\mathrm{O}_{2}$ & $\mathrm{O}_{3}$ & $\mathrm{O}_{4}$ \\
\hline 14 & $\begin{array}{l}\text { There is no one who shares my } \\
\text { interests and concerns. }\end{array}$ & $\mathrm{O}_{4}$ & $\mathrm{O}_{3}$ & $\mathrm{O}_{2}$ & $O_{1}$ \\
\hline 15 & $\begin{array}{l}\text { There is no one who really relies } \\
\text { on me for their well-being. }\end{array}$ & $\mathrm{O}_{4}$ & $\mathrm{O}_{3}$ & $\mathrm{O}_{2}$ & $O_{1}$ \\
\hline 16 & $\begin{array}{l}\text { There is a trustworthy person I } \\
\text { could turn to for advice if I were } \\
\text { having problems. }\end{array}$ & $O_{1}$ & $\mathrm{O}_{2}$ & $\mathrm{O}_{3}$ & $\mathrm{O}_{4}$ \\
\hline 17 & $\begin{array}{l}\text { I feel a strong emotional bond with } \\
\text { at least one other person. }\end{array}$ & $O_{1}$ & $\mathrm{O}_{2}$ & $\mathrm{O}_{3}$ & $\mathrm{O}_{4}$ \\
\hline 18 & $\begin{array}{l}\text { There is no one I can depend on } \\
\text { for aid if I really need it. }\end{array}$ & $\mathrm{O}_{4}$ & $\mathrm{O}_{3}$ & $\mathrm{O}_{2}$ & $O_{1}$ \\
\hline 19 & $\begin{array}{l}\text { There is no one I feel comfortable } \\
\text { talking about problems with. }\end{array}$ & $\mathrm{O}_{4}$ & $\mathrm{O}_{3}$ & $\mathrm{O}_{2}$ & $O_{1}$ \\
\hline 20 & $\begin{array}{l}\text { There are people who admire my } \\
\text { talents and abilities. }\end{array}$ & $O_{1}$ & $\mathrm{O}_{2}$ & $\mathrm{O}_{3}$ & $\mathrm{O}_{4}$ \\
\hline 21 & $\begin{array}{l}\text { I lack a feeling of intimacy with } \\
\text { another person. }\end{array}$ & $\mathrm{O}_{4}$ & $\mathrm{O}_{3}$ & $\mathrm{O}_{2}$ & $O_{1}$ \\
\hline 22 & $\begin{array}{l}\text { There is no one who likes to do } \\
\text { the things I do. }\end{array}$ & $\mathrm{O}_{4}$ & $\mathrm{O}_{3}$ & $\mathrm{O}_{2}$ & $O_{1}$ \\
\hline 23 & $\begin{array}{l}\text { There are people who I can count } \\
\text { on in an emergency. }\end{array}$ & $\mathrm{O}_{1}$ & $\mathrm{O}_{2}$ & $\mathrm{O}_{3}$ & $\mathrm{O}_{4}$ \\
\hline 24 & $\begin{array}{l}\text { No one needs me to care for } \\
\text { them. }\end{array}$ & $\mathrm{O}_{4}$ & $\mathrm{O}_{3}$ & $\mathrm{O}_{2}$ & $O_{1}$ \\
\hline
\end{tabular}

SCORING: A score for each social provision is derived such that a high score indicates that the individual is receiving that provision. Items that are asterisked should be reversed before scoring (i.e., $4=1,3=2,2=3,1=4$ ).

1. Guidance: $3^{*}, 12,16,19^{*}$

2. Reassurance of Worth: $6^{*}, 9^{*}, 13,20$

3. Social Integration: $5,8,14^{*}, 22^{*}$

4. Attachment: $2^{*}, 11,17,21^{*}$

5. Nurturance: $4,7,15^{*}, 24^{*}$

6. Reliable Alliance: $1,10^{*}, 18^{*}, 23$ 
REFERENCE: Russell, D. \& Cutrona, C.E. (1984). The provisions of social relationships and adaptation to stress. Paper presented at the meeting of the American Psychological Association, Toronto, Ontario, Canada. 


\section{$\underline{\text { Social Participation Scale }}$}

We would like to look at a list of things people sometimes do in their free time.

In the past month, how often have you done these things?

\begin{tabular}{|l|c|c|c|c|c|c|}
\cline { 2 - 7 } \multicolumn{1}{l|}{} & & $\begin{array}{c}\text { In the past month, how } \\
\text { often did you... }\end{array}$ & $\begin{array}{c}\text { Several } \\
\text { times a } \\
\text { week }\end{array}$ & $\begin{array}{c}\text { About } \\
\text { once } \\
\text { a } \\
\text { week }\end{array}$ & $\begin{array}{c}\text { Once } \\
\text { per } \\
\text { times a } \\
\text { month }\end{array}$ & $\begin{array}{c}\text { Never } \\
\text { month } \\
\text { or } \\
\text { orss } \\
\text { almost } \\
\text { never }\end{array}$ \\
\begin{tabular}{|l} 
Attend meetings of clubs, \\
or community or \\
professional organizations?
\end{tabular} & $O_{5}$ & $O_{4}$ & $O_{3}$ & $O_{2}$ & $O_{1}$ & $O_{0}$ \\
\hline $\begin{array}{l}\text { Get together or talk on the } \\
\text { phone with family } \\
\text { members? }\end{array}$ & $O_{5}$ & $O_{4}$ & $O_{3}$ & $O_{2}$ & $O_{1}$ & $O_{0}$ \\
\hline $\begin{array}{l}\text { Get together or talk on the } \\
\text { phone with friends? }\end{array}$ & $O_{5}$ & $O_{4}$ & $O_{3}$ & $O_{2}$ & $O_{1}$ & $O_{0}$ \\
\hline Work on a hobby? & $O_{5}$ & $O_{4}$ & $O_{3}$ & $O_{2}$ & $O_{1}$ & $O_{0}$ \\
\hline $\begin{array}{l}\text { Play cards, bingo, or similar } \\
\text { games? }\end{array}$ & $O_{5}$ & $O_{4}$ & $O_{3}$ & $O_{2}$ & $O_{1}$ & $O_{0}$ \\
\hline $\begin{array}{l}\text { Go out to movie, restaurant } \\
\text { or sporting event }\end{array}$ & $O_{5}$ & $O_{4}$ & $O_{3}$ & $O_{2}$ & $O_{1}$ & $O_{0}$ \\
\hline $\begin{array}{l}\text { Go out and do some } \\
\text { shopping? }\end{array}$ & $O_{5}$ & $O_{4}$ & $O_{3}$ & $O_{2}$ & $O_{1}$ & $O_{0}$ \\
\hline $\begin{array}{l}\text { Go on day trips or } \\
\text { overnight trips? }\end{array} O_{5}$ & $O_{4}$ & $O_{3}$ & $O_{2}$ & $O_{1}$ & $O_{0}$ \\
\hline Do volunteer work? & $O_{5}$ & $O_{4}$ & $O_{3}$ & $O_{2}$ & $O_{1}$ & $O_{0}$ \\
\hline $\begin{array}{l}\text { Attend religious meetings } \\
\text { or services? }\end{array}$ & $O_{5}$ & $O_{4}$ & $O_{3}$ & $O_{2}$ & $O_{1}$ & $O_{0}$ \\
\hline
\end{tabular}

Was the last month different from normal in terms of how often you did these things? No $\square$ Yes

If so, why? 


\section{Negative Interaction Scale}

INSTRUCTIONS: In answering the following questions, think about your current relationships with friends, family members, co-workers, community members and so on. Please indicate to what extent each statement describes your experience with those people in the last month.

\begin{tabular}{|l|c|c|c|c|} 
In the past month, how often have... & Never & $\begin{array}{c}\text { in a } \\
\text { while }\end{array}$ & $\begin{array}{c}\text { Fairly } \\
\text { often }\end{array}$ & $\begin{array}{c}\text { Very } \\
\text { often }\end{array}$ \\
\hline a. Have others made too many demands of you? & $\bigcirc_{0}$ & $\bigcirc_{1}$ & $\bigcirc_{2}$ & $\bigcirc_{3}$ \\
\hline b. Have others been critical of you? & $\bigcirc_{0}$ & $\bigcirc_{1}$ & $\bigcirc_{2}$ & $\bigcirc_{3}$ \\
\hline c. Have others pried into your affairs? & $\bigcirc_{0}$ & $\bigcirc_{1}$ & $\bigcirc_{2}$ & $\bigcirc_{3}$ \\
\hline d. Have others taken advantage of you? & $\bigcirc_{0}$ & $\bigcirc_{1}$ & $\bigcirc_{2}$ & $\bigcirc_{3}$ \\
\hline
\end{tabular}

SCORING: A total is created by summing the scores for the 4 items.

REFERENCE: Krause, N. (1995). Negative interaction and satisfaction with social support among older adults. Journal of Gerontology: Psychological Sciences, 50B, 5973. 
$\underline{\text { Social Self-Efficacy Scale }}$

\begin{tabular}{|c|c|c|c|c|c|c|}
\hline & & $\begin{array}{l}\text { Strongly } \\
\text { Disagree }\end{array}$ & Disagree & $\begin{array}{l}\text { Neither } \\
\text { agree } \\
\text { nor } \\
\text { disagree }\end{array}$ & Agree & $\begin{array}{l}\text { Strongly } \\
\text { Agree }\end{array}$ \\
\hline 1 & It is difficult for me to make friends. & $\mathrm{O}_{4}$ & $\mathrm{O}_{3}$ & $\mathrm{O}_{2}$ & $\mathrm{O}_{1}$ & $\mathrm{O}_{0}$ \\
\hline 2 & $\begin{array}{l}\text { If I see someone that I would like } \\
\text { to meet, I go to that person instead } \\
\text { of waiting for him or her to come to } \\
\text { me. }\end{array}$ & $O_{0}$ & $O_{1}$ & $\mathrm{O}_{2}$ & $\mathrm{O}_{3}$ & $\mathrm{O}_{4}$ \\
\hline 3 & $\begin{array}{l}\text { If I meet someone interesting who } \\
\text { is hard to make friends with, I'll } \\
\text { soon stop trying to make friends } \\
\text { with that person. }\end{array}$ & $\mathrm{O}_{4}$ & $\mathrm{O}_{3}$ & $\mathrm{O}_{2}$ & $\mathrm{O}_{1}$ & $\mathrm{O}_{0}$ \\
\hline 4 & $\begin{array}{l}\text { When I'm trying to make friends } \\
\text { who seem uninterested at first, I } \\
\text { don't give up easily. }\end{array}$ & $\mathrm{O}_{0}$ & $O_{1}$ & $\mathrm{O}_{2}$ & $\mathrm{O}_{3}$ & $\mathrm{O}_{4}$ \\
\hline 5 & $\begin{array}{l}\text { I do not handle myself well in } \\
\text { social gatherings. }\end{array}$ & $\mathrm{O}_{4}$ & $\mathrm{O}_{3}$ & $\mathrm{O}_{2}$ & $\mathrm{O}_{1}$ & $\mathrm{O}_{0}$ \\
\hline 6 & $\begin{array}{l}\text { I have acquired friends through my } \\
\text { personal abilities at making } \\
\text { friends. }\end{array}$ & $\mathrm{O}_{0}$ & $O_{1}$ & $\mathrm{O}_{2}$ & $\mathrm{O}_{3}$ & $\mathrm{O}_{4}$ \\
\hline
\end{tabular}

SCORING: Items 1, 3 and 5 are reverse-scored and then a total is calculated.

REFERENCE: Sherer, M., Maddux, J.E., Mercandante, B., Prentice-Dunn, S., Jacobs, B., \& Rogers, R.W. (1982). The self-efficacy scale: Construction and validation. Psychological Reports, 51, 663-671. 
Appendix E. Evidence for Content Validity of the CETI-M 
Table E1

Content Validity for the CETI-M: Comparison with Other Communication-Related Scales

\begin{tabular}{|c|c|c|c|c|}
\hline & CETI-M & HHIE-S & CES & VHI-F \\
\hline \multicolumn{5}{|c|}{ Participation-related concepts in survey items } \\
\hline With familiar persons (friends, family etc.) & $X$ & $X$ & $X$ & $X$ \\
\hline With unfamiliar persons & $\mathrm{X}$ & $\mathrm{X}$ & $\mathrm{X}$ & $\mathrm{X}$ \\
\hline In a quiet environment (e.g. at home) & $\mathrm{X}$ & & $\mathrm{X}$ & $\mathrm{X}$ \\
\hline In a noisy environment (e.g. a restaurant) & $\mathrm{X}$ & $X$ & $X$ & $\mathrm{X}$ \\
\hline On the phone & $\mathrm{X}$ & & $\mathrm{X}$ & $\mathrm{X}$ \\
\hline Listening to $\mathrm{TV} /$ radio & & $\mathrm{X}$ & & \\
\hline When upset/angry & & & $\mathrm{X}$ & \\
\hline During a long conversation & $\mathrm{X}$ & & & \\
\hline When someone whispers & & $X$ & & \\
\hline With children & $\mathrm{X}$ & & & \\
\hline In a group & $\mathrm{X}$ & & & $\mathrm{X}$ \\
\hline At a distance/when travelling & $\mathrm{X}$ & & $\mathrm{X}$ & \\
\hline Attending religious services & & $\mathrm{X}$ & & \\
\hline \multicolumn{5}{|l|}{ Handicap-related concepts in survey items } \\
\hline Emotional reactions to difficulties & & $\mathrm{X}$ & & \\
\hline Sense of exclusion/isolation/avoidance & & $\mathrm{X}$ & & $\mathrm{X}$ \\
\hline Feelings of handicap & & $\mathrm{X}$ & & \\
\hline Reaction of others & & $\mathrm{X}$ & & $\mathrm{X}$ \\
\hline Effort/work of communication & & & & $\mathrm{X}$ \\
\hline \multicolumn{5}{|l|}{ Unpredictability/variability/quality } \\
\hline Financial consequences & & & & $\mathrm{X}$ \\
\hline
\end{tabular}

Note. An X indicates the presence of an item relating to the that particular concept on the survey. CES = Communicative Effectiveness Survey (Donovan et al., 2008); CETI-M = Communicative Effectiveness Index-Modified (Ball et al., 2004); HHIE-S = Hearing Handicap Inventory for the Elderly Screening Version (Ventry \& Weinstein, 1983); VHI$\mathrm{F}=$ Voice Handicap Index-Functional Subscale (Jacobson et al., 1997). 
Appendix F. Open-Ended Questions for the Individual Interviews 
1. How has [particular communication impairment] impacted your daily life?

2. What has having [impairment] meant for your quality of life?

3. Some people with [particular communication impairment] say that their relationships with their friends and families changes. What has your experience been?

4. In what ways has it affected relationships with friends?

5. What about your family?

6. How has it affected your relationship with your [partner/spouse]?

7. How has it affected your ability to interact people you don't know?

8. Are there situations where you used to have trouble that you have learned to deal with over time? (If so, what/how? Examples?)

9. Are there types of situations or activities that you avoid because you experience difficulty? (If so, what/why? Examples?)

10. What advice would you have for someone dealing with [particular communication impairment]?

11. What advice would you have for their friends and family? 
Appendix G: Study Approval Documentation from OHSU and PSU 


\section{OREGON} HEALTH\&SCIENCE

Date: $\quad 5 / 30 / 2014$

To: $\quad$ Donna Graville, PhD, MS

Kathryn Schuff, MD, MCR, Chair, Institutional Review Board

Elizabeth Haney, MD, Vice-Chair, Institutional Review Board

Lynn Marshall, SCD, Vice-Chair, Institutional Review Board

From: Penny Hogarth, MD, Vice-Chair, Institutional Review Board

Kara Manning Drolet, $\mathrm{PhD}$, Associate Director, OHSU Research Integrity Office

Andrea Johnson, JD, CIP, Assistant Research Integrity Officer, Institutional Review Board

David Holmgren, MS, IRB Manager, Institutional Review Board

William Hoffman, PhD, MD, VA IRB Co-Chair

Subject: $\quad$ IRB00010500, Communication, Health, Aging, Relationship Types and Support

\section{Initial Study Approval}

The above submission was reviewed and approved for one year effective $\underline{5 / 30 / 2014}$.

\section{Review category: Expedited Category \#7}

Copies of all approved documents are available in the study's Official Documents list in the eIRB.

Ongoing IRB submission requirements:

- Six to ten weeks before the expiration date, you are to submit a continuing review to request continuing approval.

- Any changes to the project must be submitted for IRB approval prior to implementation.

- Unanticipated problems and protocol deviations must be submitted per OHSU policy.

- You are required to submit a termination request when your research is completed.

Guidelines for Study Conduct

In conducting this study, you are required to follow the guidelines in the document entitled, "Roles and Responsibilities in the Conduct of Research and Administration of Sponsored Projects," as well as all other applicable OHSU IRB Policies and Procedures.

Requirements under HIPAA

If your study involves the collection, use, or disclosure of Protected Health Information (PHI), you must comply with all applicable requirements under HIPAA. See the HIPAA and Research website and the Information Privacy and Security website for more information. 


\title{
胥 Portland State
}

\author{
$503-725-2227$ tel \\ Portland, Oregon 97207-0751 503-725-8170 fax \\ Human Subjects Research Review Committee
}

hsrrc@lists.pdx.edu

Date: June 3,2014

To: Jason Newson; Andrew Palmer

From: Karen Cellarius, HSRRC Chair

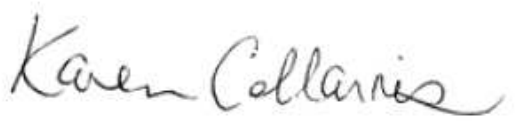

Re: $\quad$ HSRRC approval for your project titled, "The Impact of Communication Impairments on the Social Relationships of Older Adults" HSRRC Proposal \# 143059

Approval-Expiration: $6 / 3 / 14$ to $6 / 2 / 15 /$

Review Type: Expedited, Categories 6,7

In accordance with your request, the PSU Human Subjects Research Review Committee has reviewed your request for approval of the project referenced above for compliance with PSU and DHHS policies and regulations covering the protection of human subjects. The Committee is satisfied that your provisions for protecting the rights and welfare of all subjects participating in the research are adequate, and your project is approved. Please note the following requirements:

Approval: You are approved to conduct this research study only during the period of approval cited above; and the research must be conducted according to the plans and protocol submitted (approved copy enclosed).

Consent: Signed consent is required from all participants in this study.

Changes to Protocol: Any changes in the proposed study, whether to procedures, survey instruments, consent forms or cover letters, must be outlined and submitted to the Committee immediately. The proposed changes cannot be implemented before they have been reviewed and approved by the Committee.

Continuing Review: This approval will expire on June 2, 2015. It is the investigator's responsibility to ensure that a Continuing Review Report on the status of the project is submitted to the HSRRC two months before the expiration date, and that approval of the study is kept current. The Continuing Review Report is available at www.rsp.pdx.edu/compliance_human.php and in the Office of Research and Strategic Partnerships (RSP).

Adverse Reactions and/or Unanticipated Problems: If any adverse reactions or unanticipated problems occur as a result of this study, you are required to notify the Committee immediately. If the issue is serious, approval may be withdrawn pending an investigation by the Committee.

Completion of Study: Please notify the Committee as soon as your research has been completed. Study records, including protocols and signed consent forms for each participant, must be kept by the investigator in a secure location for three years following completion of the study (or per any requirements specified by the project's funding agency).

If you have questions or concerns, please contact the Office of Research Integrity in the PSU RSP at 503-725-2227. 
Appendix H. Approved Study Documents 
TITLE: Communication, Health, Aging, Relationship Types and Support

PRINCIPAL INVESTIGATOR: $\quad$ Donna Graville, $\mathrm{PhD}$

(503) $494-5947$

CO-INVESTIGATORS: $\quad$ Andrew Palmer, MS

PURPOSE: You have been invited to be in this research study because you are over the age of 65. The purpose of this study is to learn more about the health, communication status, and social relationships of a diverse group of older adults. It is anticipated that between 100-300 individuals will take part in this study. Some of these individuals may have been seen at OHSU in the past. Others may have been seen at other clinics or rehabilitation centers in the Pacific Northwest or participate in online support groups for individuals with a range of different medical conditions.

\section{PROCEDURES:}

There are two parts to this study:

- Part 1: After you fill out a written questionnaire and return it to us by mail, no additional study participation will be required after that time. The questionnaire will likely take around 15-20 minutes to complete. If you have difficulty writing and would prefer to fill out the questionnaire online, it is available at: www.surveymonkey.com/s/charts-study. If it is easier to respond to these questions verbally either in person or by phone, one of the study investigators would be happy to arrange this. Please contact Andrew Palmer for details.

- Part 2: You may also be interviewed regarding some of these same topics and how they have changed over time. This part of the study is optional and will be conducted with a smaller number of 12 individuals.

If you have any questions regarding this study now or in the future, contact Andrew Palmer, by phone (503-494-5947) or email (palmeran@ohsu.edu).

RISKS: Although we have made every effort to protect your identity, there is a minimal risk of loss of confidentiality.

BENEFITS: You may or may not benefit from being in this study. However, by serving as a subject, you may help us learn how to benefit patients in the future.

\section{CONFIDENTIALITY:}

You may choose to respond to this questionnaire anonymously. In that case, since we are not receiving any identifiable information about you, there is little chance of breach of confidentiality.

On the last page of the questionnaire there is an optional page which asks you for some identifying information with regard to two scenarios. First, if you wish to be entered into the 
drawing for a \$20 gift-card (see below). Second, if you would be willing to participate in an optional second part of the study, namely a face-to-face interview (also discussed below). If you wish to be included in either of these aspects of the study and enter your contact information, the final page of the survey will be removed from the rest of the questionnaire and stored separately so that your responses on the questionnaire are not stored with any identifying information in order to preserve your confidentiality. A 3-digit coded identifier will be the only way for the investigators to select individuals for the gift-card drawing and interviews. Once all of the study measures have been completed, this list and all identifying information will be destroyed.

In all cases, every effort will be made to protect your identity and no identifying information will be used when the study findings are published.

COSTS: It will not cost you anything to participate in this study.

COMPENSATION: Of the respondents who complete the study survey, 10 will be randomly selected to receive a $\$ 20$ gift-card. Of the respondents who participate in the face-to-face interviews, all 12 will receive a $\$ 20$ gift-card.

PARTICIPATION: If you have any questions regarding your rights as a research subject, you may contact the OHSU Research Integrity Office at (503) 494-7887.

You do not have to join this or any research study. If you do join, and later change your mind, you may quit at any time. If you refuse to join or withdraw early from the study, there will be no penalty or loss of any benefits to which you are otherwise entitled. 
The OHSU study of Communication, Health, Aging, Relationship Types and Support (CHARTS) OHSU IRB\#: 10500 Principal Investigator: Donna Graville, PhD, CCC-SLP

I would like to ask for your help! I would like to invite you to participate in a research study I am conducting in conjunction with Portland State University's Institute on Aging.

My name is Andrew Palmer and I am a Speech-Language Pathologist at OHSU. I have worked with individuals with a variety of medical conditions causing changes in their communication and health for many years. Over that time, I have become increasingly interested to learn more about how health and communication affect the lives of older adults with regard to social relationships with friends, family and acquaintances. This is a research project that I have been working on for the past several years in order get my $\mathrm{PhD}$, and I'd like your help to answer some of these questions. So, I'd like to invite you to participate in this research study.

\section{Your responses are very important and I am hoping that you will}

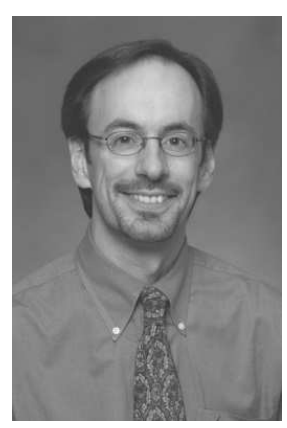
be able to find the time to participate. Enclosed in this packet is a questionnaire which I think may take around 15-20 minutes to complete. There is also a stamped, addressed envelope enclosed for you to send the completed information back to me. As I am trying to get responses from as many people as possible, your assistance would be greatly appreciated. The enrollment in the study is voluntary and all of your answers will be confidential.

I realize that your time is very precious. Unfortunately, this study is not funded by any type of grant and I do not have the ability to pay everyone for their participation. As a small token of my appreciation, however, all individuals who complete the survey will be entered into a drawing and 10 people will be randomly selected to receive a $\$ 20$ gift-card.

My goal is that at least 100 people fill out the written survey. I anticipate that we will learn a lot from these responses and that these answers may in fact generate some additional questions! As a result, I am planning to invite a smaller number of people (12) to participate in follow-up interviews. This part of the study is optional and will help to provide some more detailed information to help interpret the findings from the questionnaires.

If you have any questions or concerns about the study, please do not hesitate to contact me by mail, email or telephone. If you do not wish to participate and do not want to be contacted, you can also let me know by any of those means. If you have difficulty writing and would rather participate in the survey by phone, in person or online, this is also possible and I am happy to make these arrangements. More information about the study is available on the Web at www.ohsu.edu/charts-study

Sincerely,

Andrew Palmer, MS, CCC-SLP

Email: palmeran@ohsu.edu Web: www.ohsu.edu/charts-study

Phone: 503-494-8885

Toll-free number: 1-888-222-6478, extension 4-8885 


\section{The OHSU Study of Communication, Health, Aging, Relationship Types, and Support (CHARTS)}

\section{SECTION 1: HEALTH \& COMMUNICATION}

1. How would you describe your health at the present time?

$$
\begin{aligned}
& \square \text { Excellent } \\
& \square \text { Very good } \\
& \square \text { Good } \\
& \text { Gair } \\
& \square \text { Poor }
\end{aligned}
$$

2. Compared to most people your age, would you say your health is...?

Much better than people your age Better than people your age

About the same as people your age

Worse than people your age

Much worse than people your age

3. Compared to a year ago, how is your health today?

Much better than a year ago

Better than a year ago

About the same as a year ago

Worse than a year ago

Much worse than a year ago

The following questions ask about your ability to do different activities.

\section{Do you wear glasses?$$
\text { Y Yes }
$$$$
\text { No }
$$

5. Do you have difficulty seeing (even if wearing glasses)?

No difficulty

$\square$ Some difficulty

A lot of difficulty

Cannot do at all / unable to do

6. Do you use a hearing aid?

$$
\square \text { Yes }
$$

$\square$ No
7. Do you have difficulty hearing (even if using a hearing aid)?

$\square$ No difficulty

$\square$ Some difficulty

A lot of difficulty

- Cannot do at all / unable to do

8. Using your usual language, do you have difficulty communicating (for example understanding or being understood)?

No difficulty

Some difficulty

A lot of difficulty

Cannot do at all / unable to do

9. Do you use Sign Language?

$\square$ Yes

$\square$ No

10. Do you have difficulty remembering or concentrating?

No difficulty

Difficulty remembering only

Difficulty concentrating only

Difficulty with both

remembering and concentrating

11. How often do you have difficulty remembering? Would you say:

$\square$ Never

Sometimes

O Often

All of the time

12. Do you have difficulty remembering a few things, a lot of things, or almost everything?

$\square$ Nothing

A few things

A lot of things

Almost everything 
13. Have you ever been told by a doctor or other health professional that you had any of the following conditions?

\begin{tabular}{|c|c|c|c|}
\hline a. & High blood pressure or hypertension & Yes & No \\
\hline b. & Asthma & Yes & No \\
\hline c. & Emphysema or chronic bronchitis & Yes & No \\
\hline d. & Arthritis or rheumatism & Yes & No \\
\hline e. & Diabetes & Yes & No \\
\hline f. & Stomach or intestinal ulcers & Yes & No \\
\hline g. & Liver disease & Yes & No \\
\hline h. & Kidney or bladder problems & Yes & No \\
\hline i. & (If male) Prostate problems & Yes & No \\
\hline $\mathrm{j}$. & Cancer or a malignancy of any kind & Yes & No \\
\hline $\mathrm{k}$. & Heart attack or heart failure & Yes & No \\
\hline I. & Stroke (or any kind of disability or impairment due to a stroke) & Yes & No \\
\hline $\mathrm{m}$. & Hip fracture & Yes & No \\
\hline $\mathrm{n}$. & Parkinson's Disease & Yes & No \\
\hline o. & Amyotrophic Lateral Sclerosis (or Lou Gehrig's Disease) & Yes & No \\
\hline p. & Multiple Sclerosis & Yes & No \\
\hline q. & Spasmodic dysphonia & Yes & No \\
\hline r. & Cancer of the head and neck (inc. mouth, throat) & Yes & No \\
\hline s. & Hearing impairment / hearing loss & Yes & No \\
\hline $\mathrm{t}$. & Traumatic brain injury & Yes & No \\
\hline u. & Dementia / Alzheimer's Disease & Yes & No \\
\hline v. & Other (please list): & Yes & No \\
\hline
\end{tabular}


14. The following questions relate to any difficulties you may have because of health problems. How difficult is it for you to:

\begin{tabular}{|c|c|c|c|c|c|c|}
\hline & & $\begin{array}{l}\text { Can't } \\
\text { do at } \\
\text { all }\end{array}$ & $\begin{array}{l}\text { Very } \\
\text { difficult }\end{array}$ & $\begin{array}{l}\text { Some- } \\
\text { what } \\
\text { difficult }\end{array}$ & $\begin{array}{l}\text { Only a } \\
\text { little } \\
\text { difficult }\end{array}$ & $\begin{array}{l}\text { Not } \\
\text { difficult } \\
\text { at all }\end{array}$ \\
\hline a. & $\begin{array}{l}\text { Walk a quarter of a mile - } \\
\text { about } 3 \text { city blocks? }\end{array}$ & 0 & 1 & 2 & 3 & 4 \\
\hline b. & Climb 2 or 3 flights of stairs? & 0 & 1 & 2 & 3 & 4 \\
\hline c. & $\begin{array}{l}\text { Do work around the house } \\
\text { such as cleaning, laundry, } \\
\text { yardwork, or shoveling snow? }\end{array}$ & 0 & 1 & 2 & 3 & 4 \\
\hline d. & $\begin{array}{l}\text { Travel independently by car } \\
\text { or public transportation (e.g. } \\
\text { by bus, train, or subway)? }\end{array}$ & 0 & 1 & 2 & 3 & 4 \\
\hline e. & Use the telephone? & 0 & 1 & 2 & 3 & 4 \\
\hline f. & Manage your finances? & 0 & 1 & 2 & 3 & 4 \\
\hline g. & $\begin{array}{l}\text { Shop for food or household } \\
\text { goods? }\end{array}$ & 0 & 1 & 2 & 3 & 4 \\
\hline h. & Prepare your own meals? & 0 & 1 & 2 & 3 & 4 \\
\hline i. & $\begin{array}{l}\text { Lift or carry something as } \\
\text { heavy as } 15 \text { pounds (e.g. a } \\
\text { full bag of groceries)? }\end{array}$ & 0 & 1 & 2 & 3 & 4 \\
\hline j. & $\begin{array}{l}\text { Grasp or handle small objects } \\
\text { (e.g. a door handle or coins)? }\end{array}$ & 0 & 1 & 2 & 3 & 4 \\
\hline k. & $\begin{array}{l}\text { Get in and out of bed or a } \\
\text { chair? }\end{array}$ & 0 & 1 & 2 & 3 & 4 \\
\hline I. & Bend, kneel, or stoop? & 0 & 1 & 2 & 3 & 4 \\
\hline $\mathrm{m}$. & Bathe or dress yourself? & 0 & 1 & 2 & 3 & 4 \\
\hline $\mathrm{n}$. & $\begin{array}{l}\text { Take your medications or } \\
\text { care for your health at home? }\end{array}$ & 0 & 1 & 2 & 3 & 4 \\
\hline o. & Feed yourself? & 0 & 1 & 2 & 3 & 4 \\
\hline
\end{tabular}


15. Please evaluate how effectively you communicate in the following situations. Read the item describing the situation and then decide how successfully you communicate in that situation. If you think your communication is very effective, circle the 7 . If communication doesn't occur at all, circle the 1. Circle any number on the scale that best describes your communication in that situation.

\begin{tabular}{|c|c|c|c|c|c|c|c|c|}
\hline \multirow[b]{2}{*}{ a. } & \multirow[b]{2}{*}{$\begin{array}{l}\text { Having a conversation with familiar people } \\
\text { in a quiet environment. }\end{array}$} & \multicolumn{3}{|c|}{$\begin{array}{l}1=\text { Not at } \\
\text { all effective }\end{array}$} & \multirow[b]{2}{*}{4} & \multirow[b]{2}{*}{5} & \multicolumn{2}{|c|}{$\begin{array}{l}7 \text { = Very } \\
\text { effective }\end{array}$} \\
\hline & & 1 & 2 & 3 & & & 6 & 7 \\
\hline b. & $\begin{array}{l}\text { Having a conversation with strangers in a } \\
\text { quiet environment. }\end{array}$ & 1 & 2 & 3 & 4 & 5 & 6 & 7 \\
\hline c. & $\begin{array}{l}\text { Having a conversation with a familiar } \\
\text { person over the phone. }\end{array}$ & 1 & 2 & 3 & 4 & 5 & 6 & 7 \\
\hline d. & $\begin{array}{l}\text { Having a conversation with young } \\
\text { children. }\end{array}$ & 1 & 2 & 3 & 4 & 5 & 6 & 7 \\
\hline e. & $\begin{array}{l}\text { Having a conversation with a stranger over } \\
\text { the phone. }\end{array}$ & 1 & 2 & 3 & 4 & 5 & 6 & 7 \\
\hline f. & $\begin{array}{l}\text { Having a conversation while traveling in a } \\
\text { car. }\end{array}$ & 1 & 2 & 3 & 4 & 5 & 6 & 7 \\
\hline g. & $\begin{array}{l}\text { Having a conversation with someone at a } \\
\text { distance. }\end{array}$ & 1 & 2 & 3 & 4 & 5 & 6 & 7 \\
\hline h. & $\begin{array}{l}\text { Having a conversation with someone in a } \\
\text { noisy environment. }\end{array}$ & 1 & 2 & 3 & 4 & 5 & 6 & 7 \\
\hline i. & $\begin{array}{l}\text { Speaking or having a conversation before } \\
\text { a group. }\end{array}$ & 1 & 2 & 3 & 4 & 5 & 6 & 7 \\
\hline j. & $\begin{array}{l}\text { Having a long conversation with someone } \\
\text { (over an hour). }\end{array}$ & 1 & 2 & 3 & 4 & 5 & 6 & 7 \\
\hline
\end{tabular}

15b. Did you have any communication problems as a child? Yes No If yes, please describe: 


\section{SECTION 2: SOCIAL RELATIONSHIPS}

The next questions relate to your relationships with your FAMILY. Considering the people that you are related to by birth, marriage, adoption, etc...

16. How many relatives do you see or hear from at least once a month?
0. $]$ None
1. $\square$ One
2. Two
3. Three or four
4. Five to eight
5. Nine or more

17. How often do you see or hear from the relative with whom you have the most contact?
o. $\square$ Less than monthly
1. Monthly
2. A few times a month
3. Weekly
4. A few times a week
5. Daily

18. How many relatives do you feel at ease with that you can talk about private matters?
0. $\square$ None
1. $\square$ One
2. Two
3. Three or four
4. Five to eight
5. Nine or more

19. How many relatives do you feel close to such that you could call on them for help?
0. None
1. $\mathrm{O}$ One
2. Two
3. Three or four
4. Five to eight
5. Nine or more

20. When one of your relatives has an important decision to make, how often do they talk to you about it?
0. Never
1. Seldom
2. Sometimes
3. Often
4. Very often
5. Always

21. How often is one of your relatives available for you to talk to when you have an important decision to make?
0. $\square$ Never
1. Seldom
2. Sometimes
3. $\square$ Often
4. Very often
5. always

The next questions relate to your FRIENDSHIPS. Considering all of your friends, including those who live in your neighborhood...

22. How many of your friends do you see or hear from at least once a month?
0. None
1. $\square$ One
2. Two
3. Three or four
4. Five to eight
5. Nine or more

23. How often do you see or hear from the friend with whom you have the most contact?
0. Less than monthly
1. Monthly
2. A few times a month
3. Weekly
4. A few times a week
5. Daily 
24. How many friends do you feel at ease with that you can talk about private matters?
o. None
1. One
2. Two
3. Three or four
4. $\square$ Five to eight
5. Nine or more

25. How many friends do you feel close to such that you could call on them for help?
o. $\square$ None
1. $\square$ One
2. Two
3. Three or four
4. Five to eight
5. Nine or more

26. When one of your friends has an important decision to make, how often do they talk to you about it?
0. $\square$ Never
1. Seldom
2. Sometimes
3. Often
4. Very often
5. always

27. How often is one of your friends available for you to talk to when you have an important decision to make?
0. $\square$ Never
1. Seldom
2. Sometimes
3. Often
4. Very often
5. Always

28. In answering the following questions, think about your current relationships with friends, family members, co-workers, community members and so on. Please indicate to what extent each statement describes your current relationships with other people.

\begin{tabular}{|c|c|c|c|c|c|}
\hline & & $\begin{array}{l}\text { Strongly } \\
\text { Disagree }\end{array}$ & Disagree & Agree & $\begin{array}{l}\text { Strongly } \\
\text { Agree }\end{array}$ \\
\hline a. & $\begin{array}{l}\text { There are people I can depend on to help } \\
\text { me if I really need it. }\end{array}$ & 1 & 2 & 3 & 4 \\
\hline b. & $\begin{array}{l}\text { I feel that I do not have close personal } \\
\text { relationships with other people. }\end{array}$ & 1 & 2 & 3 & 4 \\
\hline c. & $\begin{array}{l}\text { There is no one I can turn to for guidance } \\
\text { in times of stress. }\end{array}$ & 1 & 2 & 3 & 4 \\
\hline d. & $\begin{array}{l}\text { There are people who depend on me for } \\
\text { help. }\end{array}$ & 1 & 2 & 3 & 4 \\
\hline e. & $\begin{array}{l}\text { There are people who enjoy the same } \\
\text { social activities I do. }\end{array}$ & 1 & 2 & 3 & 4 \\
\hline f. & $\begin{array}{l}\text { Other people do not view me as } \\
\text { competent. }\end{array}$ & 1 & 2 & 3 & 4 \\
\hline g. & $\begin{array}{l}\text { I feel personally responsible for the well- } \\
\text { being of another person. }\end{array}$ & 1 & 2 & 3 & 4 \\
\hline h. & $\begin{array}{l}\text { I feel part of a group of people who share } \\
\text { my attitudes and beliefs. }\end{array}$ & 1 & 2 & 3 & 4 \\
\hline i. & $\begin{array}{l}\text { I do not think other people respect my } \\
\text { skills and abilities. }\end{array}$ & 1 & 2 & 3 & 4 \\
\hline j. & $\begin{array}{l}\text { If something went wrong, no one would } \\
\text { come to my assistance. }\end{array}$ & 1 & 2 & 3 & 4 \\
\hline
\end{tabular}




\begin{tabular}{|c|c|c|c|c|c|}
\hline & & $\begin{array}{l}\text { Strongly } \\
\text { Disagree }\end{array}$ & Disagree & Agree & $\begin{array}{l}\text { Strongly } \\
\text { Agree }\end{array}$ \\
\hline k. & $\begin{array}{l}\text { I have close relationships that provide me } \\
\text { with a sense of emotional security and } \\
\text { well-being. }\end{array}$ & 1 & 2 & 3 & 4 \\
\hline I. & $\begin{array}{l}\text { There is someone I could talk to about } \\
\text { important decisions in my life. }\end{array}$ & 1 & 2 & 3 & 4 \\
\hline m. & $\begin{array}{l}\text { I have relationships where my } \\
\text { competence and skill are recognized. }\end{array}$ & 1 & 2 & 3 & 4 \\
\hline & $\begin{array}{l}\text { There is no one who shares my interests } \\
\text { and concerns. }\end{array}$ & 1 & 2 & 3 & 4 \\
\hline & $\begin{array}{l}\text { There is no one who really relies on me } \\
\text { for their well-being. }\end{array}$ & 1 & 2 & 3 & 4 \\
\hline p. & $\begin{array}{l}\text { There is a trustworthy person I could turn } \\
\text { to for advice if I were having problems. }\end{array}$ & 1 & 2 & 3 & 4 \\
\hline q. & $\begin{array}{l}\text { I feel a strong emotional bond with at } \\
\text { least one other person. }\end{array}$ & 1 & 2 & 3 & 4 \\
\hline & $\begin{array}{l}\text { There is no one I can depend on for aid if } \\
\text { I really need it. }\end{array}$ & 1 & 2 & 3 & 4 \\
\hline & $\begin{array}{l}\text { There is no one I feel comfortable talking } \\
\text { about problems with. }\end{array}$ & 1 & 2 & 3 & 4 \\
\hline t. & $\begin{array}{l}\text { There are people who admire my talents } \\
\text { and abilities. }\end{array}$ & 1 & 2 & 3 & 4 \\
\hline & $\begin{array}{l}\text { I lack a feeling of intimacy with another } \\
\text { person. }\end{array}$ & 1 & 2 & 3 & 4 \\
\hline & $\begin{array}{l}\text { There is no one who likes to do the things } \\
\text { I do. }\end{array}$ & 1 & 2 & 3 & 4 \\
\hline & $\begin{array}{l}\text { There are people who I can count on in } \\
\text { an emergency. }\end{array}$ & 1 & 2 & 3 & 4 \\
\hline & No one needs me to care for them. & 1 & 2 & 3 & 4 \\
\hline
\end{tabular}

29. In answering the following questions, think about your current relationships with friends, family members, co-workers, community members and so on. Please indicate to what extent each statement describes your experience with those people in the last month.

\begin{tabular}{|lcccc|}
\hline In the past month, how often... & $\begin{array}{c}\text { Once } \\
\text { in a } \\
\text { while }\end{array}$ & $\begin{array}{c}\text { Fairly } \\
\text { often }\end{array}$ & $\begin{array}{c}\text { Very } \\
\text { often }\end{array}$ \\
\hline a. Have others made too many demands of you? & 0 & 1 & 2 & 3 \\
b. Have others been critical of you? & 0 & 1 & 2 & 3 \\
c. Have others pried into your affairs? & 0 & 1 & 2 & 3 \\
d. Have others taken advantage of you? & 0 & 1 & 2 & 3 \\
\hline
\end{tabular}


30. The next questions relate to different kinds of things that people sometimes do in their free time. In the past month, how often have you done these things?

\begin{tabular}{|c|c|c|c|c|c|c|c|}
\hline & & Daily & $\begin{array}{l}\text { Several } \\
\text { times a } \\
\text { week }\end{array}$ & $\begin{array}{l}\text { About } \\
\text { once a } \\
\text { week }\end{array}$ & $\begin{array}{l}\text { Several } \\
\text { times a } \\
\text { month }\end{array}$ & $\begin{array}{l}\text { Once } \\
\text { per } \\
\text { month } \\
\text { or less }\end{array}$ & $\begin{array}{l}\text { Never } \\
\text { or } \\
\text { almost } \\
\text { never }\end{array}$ \\
\hline a. & $\begin{array}{l}\text { Attend meetings of clubs, or } \\
\text { community or professional } \\
\text { organizations? }\end{array}$ & 5 & 4 & 3 & 2 & 1 & 0 \\
\hline b. & $\begin{array}{l}\text { Get together or talk on the } \\
\text { phone with family members? }\end{array}$ & 5 & 4 & 3 & 2 & 1 & 0 \\
\hline c. & $\begin{array}{l}\text { Get together or talk on the } \\
\text { phone with friends? }\end{array}$ & 5 & 4 & 3 & 2 & 1 & 0 \\
\hline d. & Work on a hobby? & 5 & 4 & 3 & 2 & 1 & 0 \\
\hline e. & $\begin{array}{l}\text { Play cards, bingo, or similar } \\
\text { games? }\end{array}$ & 5 & 4 & 3 & 2 & 1 & 0 \\
\hline f. & $\begin{array}{l}\text { Go out to movie, restaurant or } \\
\text { sporting event }\end{array}$ & 5 & 4 & 3 & 2 & 1 & 0 \\
\hline g. & Go out and do some shopping? & 5 & 4 & 3 & 2 & 1 & 0 \\
\hline h. & $\begin{array}{l}\text { Go on day trips or overnight } \\
\text { trips? }\end{array}$ & 5 & 4 & 3 & 2 & 1 & 0 \\
\hline i. & Do volunteer work? & 5 & 4 & 3 & 2 & 1 & 0 \\
\hline j. & $\begin{array}{l}\text { Attend religious meetings or } \\
\text { services? }\end{array}$ & 5 & 4 & 3 & 2 & 1 & 0 \\
\hline
\end{tabular}

Was the last month different from normal in terms of how often you did these things?

$$
\text { a No } \quad \text { Yes }
$$

If so, why? 
31. The following statements relate to your ability to deal with others in social situations. How much would you agree or disagree with each of the following statements?

\begin{tabular}{lccccc|} 
& $\begin{array}{c}\text { Strongly } \\
\text { Disagree }\end{array}$ & Disagree & $\begin{array}{c}\text { Neither } \\
\text { agree } \\
\text { nor } \\
\text { disagree }\end{array}$ & Agree & $\begin{array}{c}\text { Strongly } \\
\text { Agree }\end{array}$ \\
a. It is difficult for me to make friends. & 0 & 1 & 2 & 3 & 4 \\
$\begin{array}{l}\text { If I see someone that I would like to } \\
\text { meet, I go to that person instead of } \\
\text { waiting for him or her to come to me. }\end{array}$ & 0 & 1 & 2 & 3 & 4 \\
$\begin{array}{l}\text { If I meet someone interesting who is } \\
\text { hard to make friends with, I'll soon stop } \\
\text { trying to make friends with that person. }\end{array}$ & 0 & 1 & 2 & 3 & 4 \\
$\begin{array}{l}\text { When I'm trying to make friends who } \\
\text { seem uninterested at first, I don't give } \\
\text { up easily. }\end{array}$ & 0 & 1 & 2 & 3 & 4 \\
e. I do not handle myself well in social \\
gatherings.
\end{tabular}

Would you say that these characteristics about yourself have changed over time?
口 No
口 Yes

If so, how? 
32. Below are some statements that might describe ways that you feel. For each statement please indicate how often you feel that way from "Never" to "Always."

\begin{tabular}{|lcccc|}
\hline How often do you feel... & Never & Rarely & $\begin{array}{c}\text { Some- } \\
\text { times }\end{array}$ & Always \\
\hline a. Isolated from others? & 1 & 2 & 3 & 4 \\
b. That you belong to a group of friends? & 1 & 2 & 3 & 4 \\
$\begin{array}{l}\text { c. That no one really knows you well? } \\
\text { d. That your relationships with others are not } \quad 1\end{array}$ & 2 & 3 & 4 \\
$\quad$ meaningful? & 1 & 2 & 3 & 4 \\
$\begin{array}{l}\text { e. That there are people who really understand you? } \\
\text { f. That you lack companionship? }\end{array}$ & 1 & 2 & 3 & 4 \\
\hline
\end{tabular}

33. Below is a list of the ways you might have felt or behaved. Please tell me how often you have felt this way during the past week.

\begin{tabular}{|lcccc|}
\hline During the past week... & $\begin{array}{c}\text { Rarely or } \\
\text { none of } \\
\text { the time } \\
\text { (less than } \\
\mathbf{1} \text { day) }\end{array}$ & $\begin{array}{c}\text { Some or a } \\
\text { little of } \\
\text { the time } \\
(\mathbf{1 - 2} \text { days })\end{array}$ & $\begin{array}{c}\text { Occasion- } \\
\text { ally or a } \\
\text { moderate } \\
\text { amount of } \\
\text { the time } \\
\text { (3-4 days) }\end{array}$ & $\begin{array}{c}\text { Most or } \\
\text { all of the } \\
\text { time } \\
\text { (5-7 days) }\end{array}$ \\
\hline $\begin{array}{l}\text { a. I was bothered by things that usually } \\
\text { don't bother me. }\end{array}$ & 0 & 1 & 2 & 3 \\
$\begin{array}{l}\text { b. I felt that I could not shake off the blues } \\
\text { even with help from my family or friends. } \\
\text { c. I had trouble keeping my mind on what I } \\
\text { was doing. }\end{array}$ & 0 & 1 & 2 & 3 \\
$\begin{array}{l}\text { d. I felt depressed. } \\
\text { e. I felt that everything I did was an effort. }\end{array}$ & 0 & 1 & 2 & 3 \\
f. My sleep was restless. & 0 & 1 & 2 & 3 \\
g. I was happy. & 0 & 1 & 2 & 3 \\
h. I enjoyed life. & 0 & 1 & 2 & 3 \\
i. I felt sad. & 0 & 1 & 2 & 3 \\
\hline
\end{tabular}




\section{SECTION 4: BACKGROUND INFORMATION}

34. What is your current age?

years

35. What is your gender?

Male

a Female

36. What is your current relationship status?

Married

Widowed

Separated

Divorced

Living with long-term partner

$\square$ Never married

37. What is your current employment status?

a Retired

Working full-time

Working part-time

Unemployed, looking for work

Unemployed, not looking for work

- Homemaker / Keeping house

Disabled

Other:

38. What is your highest level of education?

- High school or less

Associate / trade or vocational / some college

Four-year college degree or more

39. What is your annual household income?

- Less than $\$ 25,000$ each year

Between $\$ 25-50,000$ each year

Between $\$ 50-75,000$ each year

- Between $\$ 75-100,000$ each year

More than $\$ 100,000$ each year

Prefer not to answer
40. Do you live alone or with other people?

Live with spouse / partner

Live with other relatives / friends / room-mates

Live with paid help (e.g. caregiver)

$\square$ Live alone

Other:

41. How many people live in your household beside yourself?

people

42. What kind of residence do you currently live in?

House

Apartment

Hotel/ rooming house

a Trailer

Assisted living facility / group home

Nursing home

$\square$ Other:

43. Are you of Hispanic / Latino origin or descent?

a Yes

No

Prefer not to answer

44. Do you consider yourself to be...

White

Black / African-American

Asian

American-Indian or Alaskan Native

- Native Hawaiian / Pacific Islander

Other:

D Prefer not to answer 
PARTICIPANT ID NUMBER:

\section{CONTACT INFORMATION (OPTIONAL)}

The following information is entirely optional. If you complete this section, this final page will be removed from the rest of the survey and stored separately. The coded ID number will be the only way of connecting your answers with this information. When the study is completed all of this identifying information will be destroyed.

Would you like to be entered into the drawing for a \$20 gift card? $\quad \square$ Yes $\square$ No

If selected, would you be willing to take part in a face-to-face interview*?

* Note: The face-to-face interviews will be conducted regarding some of the same topics above, namely your health, ability to perform everyday activities, social relationships, communication, and psychological well-being, and how they have changed over time. This part of the study is optional and will be conducted with a smaller number of 12 individuals. It is estimated that they will take up to an hour. The interview will be conducted at a time \& location that is most convenient for that person. All of the interview participants will receive a $\$ 20$ gift-card.

If you answered "yes" to either question, please enter your name, address, and a contact phone or email address so we may contact you if you are selected.

Name:

Mailing address:

Phone number:

Email address (if you have one):

\section{Thank You for Taking the Time to Complete this Questionnaire!!!}

As discussed, all of the answers to these questions will be kept completely confidential.

When completed, please place the questionnaire into the postage-paid envelope and mail to: Andrew Palmer, Dept. of Otolaryngology, Mail Code: PV-01, Oregon Health \& Science University, 3181 SW Sam Jackson Park Rd., Portland, OR 97239-3098

If you have any questions about this study, the survey, or participation, please feel free to contact me directly: Phone: (503) 494-8885 Email: palmeran@ohsu.edu 
Appendix I: Identification and Exclusion of Potential Participants 


\section{Table I1}

ICD-9 Codes Used to Categorize Potential Participants into Groups A-D

\begin{tabular}{|c|c|}
\hline Group & ICD-9 codes \\
\hline \multirow{4}{*}{$\begin{array}{l}\text { A. Benign voice disorders } \\
(\mathrm{n}=253)\end{array}$} & 333.1, Vocal tremor \\
\hline & 462 , Sore throat \\
\hline & $\begin{array}{l}\text { 478.30-478.79, Vocal fold paresis/paralysis, benign mass, edema, bowing, } \\
\text { spasmodic dysphonia, \& hyperfunction }\end{array}$ \\
\hline & 784.42-784.49, Dysphonia / hypophonia \\
\hline \multirow{12}{*}{$\begin{array}{l}\text { B. Neurologic conditions } \\
(\mathrm{n}=91)\end{array}$} & 331.9, Cerebral degeneration \\
\hline & 332.0, Parkinson’s Disease \\
\hline & 333.0, Multiple Systems Atrophy \\
\hline & 334.3-334.8, Ataxia \\
\hline & 335.20-335.24, ALS \\
\hline & 340, Multiple Sclerosis \\
\hline & 356.9, Peripheral neuropathy \\
\hline & 358.01, Myasthenia Gravis \\
\hline & 359.21-359.71, Dystrophy/myositis \\
\hline & $431,434.91,437.9,438.13-438.89, \mathrm{~V} 12.54 \mathrm{CVA} /$ Effects of stroke \\
\hline & $710.3-710.4$, Myositis \\
\hline & 781.0, Dystonia \\
\hline \multirow{19}{*}{$\begin{array}{l}\text { C. Head and neck cancer } \\
(\mathrm{n}=170)\end{array}$} & 140.1, Cancer of lower lip \\
\hline & 141.0-141.9, Tongue cancer \\
\hline & 142.0, Cancer of parotid gland \\
\hline & 143.1, Cancer of lower gum \\
\hline & 144.9-145.9, Cancer of floor of mouth, buccal cavity, palate, oral cancer \\
\hline & 146.0-146.6, Tonsil cancer \\
\hline & 147.9, Nasopharynx cancer \\
\hline & 148.0-149.0, Cancer of hypopharynx \\
\hline & 150.0-150.9, Cancer of cervical esophagus \\
\hline & 160.0, Cancer of nasal cavity \\
\hline & 161.0-161.9, Laryngeal cancer \\
\hline & 171.0-173.92, Sarcoma of neck / squamous cell carcinoma \\
\hline & 193, Thyroid cancer \\
\hline & 195.0-198.89, Cancer of jaw, lymph nodes, or neck \\
\hline & 235.6, Laryngeal neoplasm \\
\hline & 526.89 , Osteoradionecrosis of jaw \\
\hline & 784.41, Aphonia \\
\hline & V15.29, History of parathyroidectomy \\
\hline & V55.0, Attention to tracheostomy \\
\hline \multirow{4}{*}{$\begin{array}{l}\text { D. Hearing impairment } \\
(\mathrm{n}=679)\end{array}$} & 389.03-389.06, Conductive hearing loss \\
\hline & 389.10-389.18, Sensorineural hearing loss \\
\hline & 389.20-389.22, Mixed hearing loss \\
\hline & 389.9, Unspecified hearing loss \\
\hline TOTAL $(n=1,193)$ & \\
\hline
\end{tabular}


Table I2

Reasons for Exclusion of Potential Participants from Groups A-D

\begin{tabular}{lcc}
\hline Reason for exclusion & $\mathrm{n}$ & $\%$ \\
\hline Deceased & 14 & 9.52 \\
Cognitive impairment & 36 & 24.49 \\
Childhood onset of communication impairment & 7 & 4.76 \\
Cancer in last year/at last visit/now & 46 & 31.29 \\
Non-native English speaker & 12 & 8.16 \\
Resident in a facility & 3 & 2.04 \\
Unable to communicate adequately for study & 11 & 7.48 \\
Individual lives outside Pacific NW & 6 & 4.08 \\
Other & 12 & 12.24 \\
\hline
\end{tabular}

Note. Of the 12 individuals in the "other" category, reasons for exclusion were as follows: there was inadequate medical information in the chart review because the patient had not been seen by a physician at OHSU $(n=5)$; the individual's diagnosis was not consistent with that of the other individuals in that category $(\mathrm{n}=5)$; or, the etiology of the problem and the individual's medical diagnosis was unknown $(\mathrm{n}=$ 2).

\section{Table I3}

Reasons for Exclusion of Potential Participants from Group E

\begin{tabular}{lrr}
\hline \multicolumn{1}{c}{ Reason for exclusion } & $\mathrm{n}$ & \multicolumn{1}{c}{$\%$} \\
\hline Deceased & 5 & 3.70 \\
Cognitive impairment & 11 & 8.15 \\
Cancer in last year/at last visit/now & 29 & 21.48 \\
Non-native English speaker & 10 & 7.41 \\
Resident in a facility & 1 & 0.74 \\
Unable to communicate adequately for study & 1 & 0.74 \\
Documented communication impairment & 63 & 46.67 \\
Individual lives outside Pacific NW & 4 & 2.96 \\
Other & 11 & 11.11 \\
\hline
\end{tabular}

Note. Of the 11 individuals who were excluded for "other" reasons, these included: the individual had not been seen by an MD and/or there was insufficient information in the medical record to determine if the individual met eligibility criteria $(\mathrm{n}=10)$; and, one individual had been placed on hospice at their last visit. 
Appendix J: Categorization of Open-Ended Responses from Phase 1 
Table J1

Categorized Sample Comments Regarding Reasons for Changes in Social Participation in the Last Month

\begin{tabular}{|c|c|}
\hline Category & Sample comments \\
\hline Communication & $\begin{array}{l}\text { - A problem with my prosthesis [communication device] required } \\
\text { some investigation and trial-and-error type resolution. } \\
\text { - I cannot be heard at ballgames so only go with my husband. } \\
\text { Also I have stopped going to parties, funerals homes, shiva } \\
\text { houses and such for the same reason. }\end{array}$ \\
\hline Health of self or others & $\begin{array}{l}\text { My husband was in and out of the hospital and nursing home } \\
\text { and I waited on him a lot. } \\
\text { - Wife's health decline as well as my own. Failing eyesight and } \\
\text { dizziness. } \\
\text { - A fall resulting in a fractured hip took me out of my normal } \\
\text { routine as I recuperated. } \\
\text { My daughter was diagnosed with lung cancer. Took a road trip } \\
\text { from Oregon to Los Angeles. I did not drive. We were busy } \\
\text { getting her house clean \& get groceries for when she came } \\
\text { home from hospital. }\end{array}$ \\
\hline Death of spouse & - $\quad$ My wife died 5/28/14 \\
\hline Personal choice & $\begin{array}{l}\text { Made more of an effort to seek out new areas to explore, such } \\
\text { as new events and new museums. } \\
\text { I have lost interest in Church and church activities so no longer } \\
\text { attend. }\end{array}$ \\
\hline Relocation & $\begin{array}{l}\text { - In the process of selling our home, moving my family and two } \\
\text { dogs, to a townhome, then beginning building a new home in } \\
\text { another town. }\end{array}$ \\
\hline Seasonal variations & $\begin{array}{l}\text { During the summer month, some of the groups I normally } \\
\text { attend are not meeting. I would normally have more meetings, } \\
\text { outings and social interaction. }\end{array}$ \\
\hline $\begin{array}{l}\text { Travel / vacation / } \\
\text { family activities }\end{array}$ & $\begin{array}{l}\text { - Travelled to another state with a friend to help her bring her } \\
\text { parents to Oregon. We ate out at restaurants more frequently } \\
\text { than I usually do. } \\
\text { - My wife and I (and some friends from Memphis) were in } \\
\text { British Columbia, Canada for about two weeks "seeing the } \\
\text { sights." Given that we're retired, I don't call that vacationing. } \\
\text { We tend to travel a lot, something I deem important to do given }\end{array}$ \\
\hline
\end{tabular}




\begin{tabular}{|c|c|}
\hline & $\begin{array}{l}\text { the fact my PD may make it difficult at some point. } \\
\text { - I spent two weeks camping in Maine with my RV }\end{array}$ \\
\hline $\begin{array}{l}\text { Work-related or } \\
\text { volunteer activities }\end{array}$ & $\begin{array}{l}\text { I'm still working at age } 80 \text {. Weekends are pretty much taken up } \\
\text { with household chores, maintenance, and reading. I expect } \\
\text { when I retire in } 4 \text { months the above responses would be } \\
\text { different. } \\
\text { - I was laid off } 45 \text { days ago. } \\
\text { - I am a voting poll worker and I volunteered } 6 \text { days in the past } 9 \\
\text { days. } \\
\text { - Attended RV races for } 4 \text { days \& worked in an information } \\
\text { booth total of } 16 \text { hours. }\end{array}$ \\
\hline
\end{tabular}




\section{Table J2}

Sample Comments from those who Report Increased Social Self-Efficacy by Category

\begin{tabular}{|c|c|}
\hline Category & Sample comments \\
\hline Communication & $\begin{array}{l}\text { - Having a cochlear implant has made me for sociable more often. I've } \\
\text { never been unsociable but did withdraw somewhat from situations } \\
\text { where I couldn't hear. }\end{array}$ \\
\hline Health & $\begin{array}{l}\text { - It has become easier after having suffered through cancer and I have } \\
\text { become more tolerant of others foibles. }\end{array}$ \\
\hline $\begin{array}{l}\text { Changed } \\
\text { priorities }\end{array}$ & $\begin{array}{l}\text { The older I get, the more I value my time and prioritize how I spend } \\
\text { my days. I'm less likely to socialize with people I don't admire or } \\
\text { enjoy. I have improved my ability to say no when asked to spend time } \\
\text { with people who don't like me or my values. Life is shorter than ever! } \\
\text { And that's a good thing! Only wish I'd known myself this well thirty or } \\
\text { forty years ago.... }\end{array}$ \\
\hline $\begin{array}{l}\text { Self- } \\
\text { confidence/ } \\
\text { maturity }\end{array}$ & $\begin{array}{l}\text { - I have become less concerned about what others think of me \& more } \\
\text { of a caring person towards others. I have a degree of social phobia in } \\
\text { large groups but have overcome my anxiety in certain circumstances. }\end{array}$ \\
\hline Social context & $\begin{array}{l}\text { - I was very backward as a child and as I lived on a ranch way out in the } \\
\text { country I didn't get together with friends often. Now that I live in a city } \\
\text { and belong to several organizations I am very welcome and do enjoy } \\
\text { many great get-togethers. Life is happy and wonderful. }\end{array}$ \\
\hline Social skills & $\begin{array}{l}\text { People think I am an extrovert. I am not, but I have learned how to put } \\
\text { on a facade and play a role. I play that role well and tend to do well at } \\
\text { social occasions (including cocktail parties where I know no one and } \\
\text { am free to circulate...) In short, I have taught myself over the years } \\
\text { how to be sociable. }\end{array}$ \\
\hline
\end{tabular}


Table J3

Sample Comments from those who Report Decreased Social Self-Efficacy by Category

\begin{tabular}{|c|c|}
\hline Category & Sample comments \\
\hline Communication & $\begin{array}{l}\text { - Spasmodic Dysphonia has changed my life. The inability to speak or } \\
\text { speak clearly has shattered my confidence. My voice is very difficult } \\
\text { to understand and speaking in a group is impossible if there is any } \\
\text { background noise. Speaking on the telephone is very difficult and } \\
\text { nearly all my family live } 2000 \text { miles away from me - e-mail and } \\
\text { texting is my communication. Unfortunately I have almost no } \\
\text { communication with my } 94 \text { year old mother who cannot hear me, does } \\
\text { not use either e-mail or texting. I avoid attending many group } \\
\text { activities. }\end{array}$ \\
\hline Health & $\begin{array}{l}\text { - I have always been a little shy but since getting Parkinson's I have to } \\
\text { use a cane to get around so I don't socialize much. }\end{array}$ \\
\hline $\begin{array}{l}\text { Changed } \\
\text { priorities }\end{array}$ & $\begin{array}{l}\text { I had many friends when younger. My friends were only friends with } \\
\text { each other because they were friends of mine. I was the glue that held } \\
\text { my friends together. Currently I find it difficult to make new friends. I } \\
\text { rely on my family for friendship and help. I tend to keep to myself. }\end{array}$ \\
\hline Social context & $\begin{array}{l}\text { I have moved west 2-1/2 years ago. Making friends very hard at my } \\
\text { age. I get along well with co-workers but am no longer working. } \\
\text { Husband retired so much of my time take up both positive and } \\
\text { negative. }\end{array}$ \\
\hline Social skills & $\begin{array}{l}\text { Verbal communication is not physically or emotionally as easy as it } \\
\text { was prior to my laryngectomy. My daily goal is to put step out of the } \\
\text { box and chat with someone. It's not easy, but by volunteering etc I am } \\
\text { pushing myself to socialize and communicate, both things that are very } \\
\text { necessary to my emotional health and happiness. After } 21 / 2 \text { years I } \\
\text { will admit I am getting more confident and comfortable accepting my } \\
\text { disability. }\end{array}$ \\
\hline Other & $\begin{array}{l}\text { - Used to be easier. I also stopped drinking alcohol which has put some } \\
\text { people off and has caused me to be disinclined to participate in } \\
\text { activities where there will be a lot of drinking. }\end{array}$ \\
\hline
\end{tabular}


Appendix K. Additional Descriptive Data from Phase 1 


\section{Table K1}

Health Conditions Reported by Study Participants with Comparative Data from the Later Life Study of Social Exchanges (LLSSE)

\begin{tabular}{lcc}
\hline & $\begin{array}{c}\text { Current Study } \\
\text { All groups (\%) }\end{array}$ & $\begin{array}{c}\text { LLSSE Study } \\
\text { Wave 1 (\%) }\end{array}$ \\
\hline High blood pressure/hypertension & 52.30 & 55.45 \\
Arthritis/rheumatism & 45.11 & 59.50 \\
Any cancer & 40.85 & 13.71 \\
Other & 33.15 & 34.70 \\
Kidney/bladder problems & 17.30 & 17.07 \\
Asthma & 14.83 & 8.08 \\
Diabetes & 13.14 & 15.84 \\
Heart attack/heart failure & 11.39 & 19.67 \\
Emphysema/chronic bronchitis & 10.59 & 7.89 \\
Stomach/intestinal ulcers & 7.63 & 9.54 \\
Stroke (or disability due to stroke) & 6.78 & 7.36 \\
Hip fracture & 3.81 & 4.72 \\
Liver disease & 2.13 & 2.08 \\
\hline
\end{tabular}




\section{Table K2}

Other Health Conditions and Procedures Reported by at Least 1\% of Study Participants

\begin{tabular}{lcc}
\hline Condition & $\mathrm{n}$ & $\%$ \\
\hline Hearing impairment / hearing loss & 84 & 35.00 \\
Head and neck cancer (any) & 68 & 28.33 \\
Prostate problems / surgery & 53 & $22.08^{\mathrm{a}}$ \\
Parkinson's Disease & 27 & 11.25 \\
Neurologic disease-Other (inc. ataxia, epilepsy/seizures, MD, MG, MS, PLS) & 19 & 7.92 \\
Cardiac or vascular issues / surgery & 19 & 7.92 \\
GI issues (inc. IBS, GERD, Barrett's esoph., dysphagia, Sjogren's, pancreatitis) & 15 & 6.25 \\
Reduced mobility and/or surgery of lower limb (hip, knee, ankle, foot) & 12 & 5.00 \\
Vision problems (inc. cataract, glaucoma, macular degeneration) & 10 & 4.17 \\
Spasmodic Dysphonia & 7 & 2.92 \\
Allergies / sinus problems & 7 & 2.92 \\
Osteoporosis & 6 & 2.50 \\
COPD & 5 & 2.08 \\
Laryngeal surgery & 5 & 2.08 \\
Thyroid problems & 5 & 2.08 \\
Cochlear implant & 4 & 1.67 \\
Depression & 3 & 1.25 \\
Miscellaneous injuries & 3 & 1.25 \\
\hline
\end{tabular}

Note. Categories are not mutually exclusive. Abbreviations: COPD = Chronic Obstructive Pulmonary Disease; GERD = Gastroesophageal Reflux Disease; $\mathrm{MD}=$ Muscular Dystrophy; $\mathrm{MG}=$ Myasthenia Gravis; MS = Multiple Sclerosis; PLS = Primary Lateral Sclerosis;

${ }^{\text {a }}$ Percentage of whole sample. Problem present in $53 / 120=44.17 \%$ of males. 


\section{Table K3}

Functional Limitations for All Participants

\begin{tabular}{lcccc}
\hline & $\begin{array}{c}\text { Any } \\
\text { difficulty }\end{array}$ & & & \\
& $(\%)$ & $M$ & $S D$ & Range \\
Activity & 34.7 & .69 & 1.06 & $0-3$ \\
\hline a. Walk a quarter of a mile - about 3 city blocks? & 51.1 & .92 & 1.07 & $0-3$ \\
b. Climb 2 or 3 flights of stairs? & 38.6 & .65 & .95 & $0-3$ \\
c. Do work around the house such as cleaning, & & & & \\
$\quad$ laundry, yardwork, or shoveling snow? & 17.0 & .29 & .74 & $0-3$ \\
d. Travel independently by car or public & & & & \\
$\quad$ transportation (e.g. by bus, train, or subway)? & 25.7 & .47 & .91 & $0-3$ \\
e. Use the telephone? & 9.4 & .13 & .45 & $0-3$ \\
f. Manage your finances? & 14.5 & .20 & .55 & $0-3$ \\
g. Shop for food or household goods? & 12.9 & .20 & .59 & $0-3$ \\
h. Prepare your own meals? & 30.5 & .53 & .91 & $0-3$ \\
i. Lift or carry something as heavy as 15 pounds & & & & \\
$\quad$ (e.g. a full bag of groceries)? & 20.7 & .30 & .66 & $0-3$ \\
j. Grasp or handle small objects (e.g. a door handle & & & & \\
$\quad$ or coins)? & 25.8 & .36 & .69 & $0-3$ \\
k. Get in and out of bed or a chair? & 50.6 & .75 & .91 & $0-3$ \\
1. Bend, kneel, or stoop? & 11.9 & .19 & .59 & $0-3$ \\
m. Bathe or dress yourself? & 8.1 & .12 & .46 & $0-3$ \\
n. Take your medications or care for your health at & & & & \\
$\quad$ home? & 4.3 & .06 & .28 & $0-3$ \\
o. Feed yourself? & & & & \\
\hline
\end{tabular}


Table K4

Social Network Scores Compared to Other Previous Studies of Older Adults

\begin{tabular}{lccc}
\hline & $\begin{array}{c}\text { Current Study } \\
(\mathrm{n}=240)\end{array}$ & $\begin{array}{c}\text { Wells (2009) } \\
(\mathrm{n}=109)\end{array}$ & $\begin{array}{c}\text { Anyanwu et al. (2011) } \\
(\mathrm{n}=512)\end{array}$ \\
\hline Social Network-Family & $18.07 \pm 5.94$ & $19.8 \pm 5.9$ & $17.5 \pm 6.2$ \\
Social Network-Friends & $16.13 \pm 5.66$ & $17.4 \pm 6.1$ & $13.0 \pm 7.0$ \\
Social Network-Total & $34.23 \pm 9.79$ & $37.2 \pm 10.3$ & $30.5 \pm 10.5$ \\
\hline
\end{tabular}

Table K5

Social Provisions Scale Scores Compared to Previously Published Values

\begin{tabular}{lcc}
\hline & $\begin{array}{c}\text { Current Study } \\
(\mathrm{n}=240)\end{array}$ & $\begin{array}{c}\text { Cutrona et al. (1986) } \\
(\mathrm{n}=50)\end{array}$ \\
\hline SPS1-Guidance & $13.98 \pm 2.06$ & $13.0 \pm 1.9$ \\
SPS2-Reassurance of worth & $13.82 \pm 1.96$ & $12.4 \pm 2.0$ \\
SPS3-Social integration & $13.50 \pm 2.19$ & $13.0 \pm 1.7$ \\
SPS4-Attachment & $13.42 \pm 2.32$ & $12.7 \pm 2.2$ \\
SPS5-Opportunity for nurturance & $12.53 \pm 2.74$ & $12.3 \pm 2.4$ \\
SPS6-Reliable alliance & $14.39 \pm 2.05$ & $13.7 \pm 1.6$ \\
SPS-Total & $81.69 \pm 10.31$ & $76.9 \pm 9.2$ \\
\hline
\end{tabular}

Table K6

Negative Interactions Scale Scores Compared to Previously Published Values

\begin{tabular}{lcc}
\hline & $\begin{array}{c}\text { Current Study } \\
(\mathrm{n}=240)\end{array}$ & $\begin{array}{c}\text { Krause (1995) } \\
(\mathrm{n}=935)\end{array}$ \\
\hline NIS1-Others make too many demands & $.74 \pm .73$ & $.57 \pm .82$ \\
NIS2-Others are critical & $.76 \pm .61$ & $.48 \pm .74$ \\
NIS3-Others pry into affairs & $.30 \pm .53$ & $.34 \pm .68$ \\
SPS4-Others take advantage & $.44 \pm .64$ & $.38 \pm .73$ \\
NIS-Total & $2.24 \pm 1.83$ & $\mathrm{NR}$ \\
\hline Note. Scores were adapted from previously published values for the purposes of comparison. In the current \\
study responses were scored from 0-3 rather than 1-4 as used by Krause.
\end{tabular}




\section{Table K7}

Scores for Items on the Social Participation Measure in Order of Frequency

\begin{tabular}{lccc}
\hline Activity & $M$ & $S D$ & Range \\
\hline Get together or talk on phone-family & 2.95 & 1.41 & $0-5$ \\
Work on a hobby & 2.88 & 1.77 & $0-5$ \\
Go shopping & 2.86 & 1.18 & $0-5$ \\
Get together or talk on phone-friends & 2.65 & 1.49 & $0-5$ \\
Go to movie/restaurant/sports event & 2.19 & 1.28 & $0-5$ \\
Do volunteer work & 1.51 & 1.62 & $0-5$ \\
Attend meetings (clubs/organizations) & 1.50 & 1.38 & $0-5$ \\
Attend religious meetings/services & 1.27 & 1.54 & $0-5$ \\
Go on day/overnight trips & 1.25 & 1.00 & $0-5$ \\
Play cards/bingo/games & 1.24 & 1.60 & $0-5$ \\
\hline
\end{tabular}

Table K8

Scores for Items on the Social Self-Efficacy Scale and the Total Score

\begin{tabular}{|c|c|c|c|}
\hline & $M$ & $S D$ & Range \\
\hline 1. It is difficult for me to make friends. & 2.81 & 1.06 & $0-4$ \\
\hline $\begin{array}{l}\text { 2. If I see someone that I would like to meet, I go to that } \\
\text { person instead of waiting for him or her to come to me. }\end{array}$ & 2.48 & 1.03 & $0-4$ \\
\hline $\begin{array}{l}\text { 3. If I meet someone interesting who is hard to make friends } \\
\text { with, I'll soon stop trying to make friends with that } \\
\text { person. }\end{array}$ & 1.79 & .94 & $0-4$ \\
\hline $\begin{array}{l}\text { 4. When I'm trying to make friends who seem uninterested at } \\
\text { first, I don't give up easily. }\end{array}$ & 2.00 & .92 & $0-4$ \\
\hline 5. I do not handle myself well in social gatherings. & 2.76 & 1.06 & $0-4$ \\
\hline $\begin{array}{l}\text { 6. I have acquired friends through my personal abilities at } \\
\text { making friends. }\end{array}$ & 2.68 & .99 & $0-4$ \\
\hline Total Social Self-Efficacy score & 14.56 & 3.66 & $2-24$ \\
\hline
\end{tabular}

